\title{
Zur Wasserstoff-induzierten Riss- und Blisterbildung in Eisen
}

\author{
Dissertation \\ zur Erlangung des mathematisch-naturwissenschaftlichen \\ Doktorgrades \\ "Doctor rerum naturalium" \\ der Georg-August-Universität Göttingen \\ im Promotionsprogramm ProPhys \\ der Georg-August University School of Science (GAUSS)
}

vorgelegt von

Marie Christine Tiegel

aus Rotenburg / Wümme

Göttingen, 2016 


\section{Betreuungsausschuss:}

Prof. Dr. Reiner Kirchheim

Institut für Materialphysik

Georg-August-Universität Göttingen

Prof. Dr. Astrid Pundt

Institut für Materialphysik

Georg-August-Universität Göttingen

\section{Mitglieder der Prüfungskommission:}

Referent:

Prof. Dr. Reiner Kirchheim

Institut für Materialphysik

Georg-August-Universität Göttingen

Korreferent:

Prof. Dr. Michael Seibt

IV. Physikalisches Institut

Georg-August-Universität Göttingen

weitere Mitglieder der Prüfungskommission:

Prof. Dr. Astrid Pundt

Institut für Materialphysik

Georg-August-Universität Göttingen

Prof. Dr. Hans Hofsäss

II. Physikalisches Institut

Georg-August-Universität Göttingen

Prof. Dr. Vasily Moshnyaga

I. Physikalisches Institut

Georg-August-Universität Göttingen

Prof. Dr. Wolfram Kollatschny

Institut für Astrophysik

Georg-August-Universität Göttingen

Tag der mündlichen Prüfung: 20.01.2017 


\section{Inhaltsverzeichnis}

$\begin{array}{lll}\text { Kapitel } 1 \quad \text { Einleitung } & 1\end{array}$

1.1. Motivation . . . . . . . . . . . . . . . . . . . . . 1

1.2. Hintergrund . . . . . . . . . . . . . . . . . 2

1.3. Fragestellungen der Arbeit . . . . . . . . . . . . . . . . 3

Kapitel 2 Theorie und Hintergrund 5

2.1. Wasserstoff in Eisen . . . . . . . . . . . . . . . . . . 5

2.1.1. Löslichkeit von Wasserstoff in Eisen . . . . . . . . . . . . . . . 5

2.1.2. Position von Wasserstoff im Eisengitter . . . . . . . . . . . . . . . 6

2.1.3. Diffusion von Wasserstoff in Eisen . . . . . . . . . . . . . . . . 7

2.1.4. Wechselwirkung von Wasserstoff mit Gitterdefekten . . . . . . . . . 7

2.2. Verformung und Bruch . . . . . . . . . . . . . . . 8

2.2.1. Verformung in krz Materialien . . . . . . . . . . . . . . . 8

2.2.2. Spröder und duktiler Bruch . . . . . . . . . . . . . . . . . . . 11

2.2.3. Linear elastische Bruchmechanik . . . . . . . . . . . . . . . 14

2.3. Wasserstoffversprödung . . . . . . . . . . . . . . . 15

2.3.1. Hydrid-Bildung und resultierender spröder Bruch . . . . . . . . . . 16

2.3.2. Herabsetzung der Kohäsion durch Wasserstoff (HEDE) . . . . . . . 17

2.3.3. Erhöhung der lokalen Plastizität durch Wasserstoff (HELP) . . . . . 19

Kapitel 3 Experimentelle Methoden $\quad 21$

3.1. Elektrochemische Wasserstoffbeladung . . . . . . . . . . . . . . . 21

3.2. Das Rasterelektronenmikroskop (REM) . . . . . . . . . . . . . . . 22

3.3. Focused Ion Beam (FIB) zur Herstellung von TEM-Lamellen . . . . . . . . 24

3.4. Transmissionselektronenmikroskop (TEM) mit verwendeter Rotationskali-

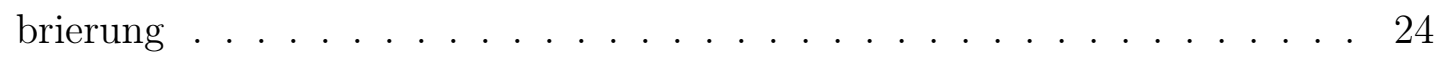

3.5. Wasserstoffanalyse . . . . . . . . . . . . . . 25

3.6. Dichtemessung zur Bestimmung des Gesamtvolumens der Risse . . . . . . 26

3.7. Messung des Wasserstoffaustritts aus der Probe nach Beladung . . . . . . . 28

3.8. Permeationsmessungen mittels eines KircTec Sensors . . . . . . . . . . . 30

3.9. Weitere verwendete Messmethoden . . . . . . . . . . . . . . 30

3.10. Finite-Elemente Simulationen . . . . . . . . . . . . . . . . . 31 


\section{Kapitel 4 Präparation und Charakterisierung der verwendeten} Eisenproben

4.1. Chemische Zusammensetzung der Proben . . . . . . . . . . . . . . 33

4.2. Probenpräparation und resultierende Mikrostruktur der Proben . . . . . . 34

$\begin{array}{lll}\text { Kapitel } 5 & \text { Ergebnisse } & 37\end{array}$

5.1. Experimentelle Ergebnisse . . . . . . . . . . . . . . . . . 37

5.1.1. Erscheinungsbild der Proben nach der Wasserstoffbeladung . . . . . 37

5.1.2. Rasterelektronenmikroskopische Untersuchung . . . . . . . . . . . . 38

5.1.3. EDX-Untersuchung der Einschlüsse im REM . . . . . . . . . . . . . 46

5.1.4. Kristallographische Orientierung mittels EBSD im REM . . . . . . 47

5.1.5. Blisterwachstum während der Wasserstoffbeladung . . . . . . . . 51

5.1.6. TEM-Untersuchung der Defektstruktur . . . . . . . . . . . . . 53

5.1.7. Einfluss durch Veränderung des Materials . . . . . . . . . . . . . 62

5.1.8. Wasserstoffgehalt der Proben . . . . . . . . . . . . . . 62

5.1.9. Messung des Wasserstoffdrucks in den Rissen . . . . . . . . . . . . . 65

5.1.10. Wasserstoff Permeation . . . . . . . . . . . . . . . . 68

5.1.11. Zugversuche . . . . . . . . . . . . . . . . . 70

5.2. Finite-Elemente Simulation der Spannung um Risse . . . . . . . . . . . . . 72

5.2.1. Spannungsintensitätsfaktor . . . . . . . . . . . . . . . . . . . 72

5.2.2. Simulation eines oberflächennahen Risses . . . . . . . . . . . . . . . 74

$\begin{array}{lll}\text { Kapitel } 6 & \text { Diskussion } & 75\end{array}$

6.1. Initiierungsstellen der Wasserstoff induzierten- Risse . . . . . . . . . . . . 75

6.2. Chemische Zusammensetzung und Form der Einschlüsse . . . . . . . . . . 78

6.3. Einfluss des Reinheitsgrades der Proben . . . . . . . . . . . . . . 79

6.4. Bildung eines Spalts zwischen Einschlüssen und umgebender Eisenmatrix . 81

6.5. Der Wasserstoffdruck als Triebkraft für die Rissbildung . . . . . . . . . . . 82

6.6. Diskontinuierlicher Rissfortschritt durch das Material . . . . . . . . . . . . 85

6.7. Rissfortschritt durch Quasispaltbruch . . . . . . . . . . . . 86

6.8. Mikrostruktur . . . . . . . . . . . . . . . . 87

$\begin{array}{lll}\text { Kapitel } 7 & \text { Zusammenfassung } & 91\end{array}$ 
Inhalt

Anhang

Kapitel A Zusätzliche Messungen $\quad 95$

A.1. Bruchfläche von ARMCO-Eisen . . . . . . . . . . . . . . . . . . . . 95

A.2. Zugversuch von ARMCO-Eisen . . . . . . . . . . . . . . . . . 97

Kapitel B Liste der Veröffentlichungen

Kapitel C Danksagung

Kapitel D Lebenslauf

\section{Literatur}




\section{\begin{tabular}{l|l} 
& \\
Einleitung & 1
\end{tabular} \\ \begin{tabular}{l|l} 
Motivation & 1.1
\end{tabular}}

Frühzeitiges Werkstoffversagen von Metallen, insbesondere von Stahl, durch den Einfluss von Wasserstoff kann in den verschiedensten Anwendungen auftreten. Ein Beispiel sind Getriebelager von Windkraftanlagen [Eva12]. Hier werden Schmieröle und Wasser an der durch die Rollreibung des Lagers freigelegten Eisenfläche aufgespalten. Der entstehende Wasserstoff kann in die Bauteile diffundieren und so Rissbildung hervorrufen [ERWW13]. Ein weiteres Beispiel sind Bohrköpfe in der Öl- und Gasförderung [PGL $\left.{ }^{+} 13\right]$, denn im geförderten Öl ist Schwefelwasserstoff gelöst, vor allem wenn das Öl aus großen Tiefen gefördert wird. In einer Reaktion von Schwefelwasserstoff, Wasser und dem Metall entsteht Wasserstoff und dieser kann die Bohrköpfe angreifen.

In den genannten Beispielen entsteht Wasserstoff während der Anwendung. Außerdem soll Wasserstoff in Zukunft als Energiespeicher für Strom aus erneuerbaren Energien oder als Treibstoff genutzt werden [Sto16, Eur03, MG12]. Wasserstoff ist dafür ein idealer Kandidat, da er im Vergleich mit anderen Kraftstoffen den höchsten spezifischen Brennwert aufweist, und bei seiner Verbrennung lediglich Wasser entsteht [Züt04]. Dass der Wasserstoff bisher kaum eingesetzt wird, hat den einfachen Grund, dass er auf der Erde nur in Verbindungen vorliegt und zunächst zum Beispiel durch Elektrolyse von Wasser zu Wasserstoffgas umgewandelt werden muss. Jedoch werden zukünftig fossile Energieträger knapper, darüber hinaus wird eine Reduzierung der Treibhausgase angestrebt [LP16]. Wasserstoff kann hier als Treibstoff Abhilfe schaffen, sofern er mit Hilfe von erneuerbaren Energiequellen gewonnen wird. Bei der Verwendung erneuerbarer Energie-Systeme kommt es zeitweise zur Überproduktion von Strom [BW12]. Dieser Strom kann schon heute teilweise nicht genutzt werden und bei einem Ausbau der erneuerbaren Energien wird sich dieses noch verschärfen. Eine Lösung wäre, gerade nicht benötigten Strom zur Wasserstoffproduktion durch Elektrolyse zu nutzen und somit Treibstoff herzustellen.

Bei einem Ausbau der Nutzung von Wasserstoff als Energieträger wird es eine Vielzahl an Bauteilen, wie Wasserstoffspeicher, aber zum Beispiel auch Leitungen geben, die in Kontakt mit Wasserstoff kommen. Dabei muss vor allem die Sicherheit über die Lebenszeit dieser Bauelemente gewährleistet sein. Dazu ist es notwendig, das frühzeitige Werkstoffversagen durch Wasserstoff zu verhindern. Deshalb ist die Erforschung des Mechanismus 
der Wasserstoffversprödung und der Wasserstoff-induzierten Rissbildung, wie ihn diese Dissertation behandelt, idealerweise bis auf atomare Skala nötig.

\section{\begin{tabular}{l|l} 
Hintergrund & 1.2
\end{tabular}}

1874 beschrieb Johnson [Joh74] erstmals, dass durch gelösten Wasserstoff in Eisen eine deutliche Veränderung der mechanischen Eigenschaften auftritt. Er stellte eine Versprödung des Materials fest, wenn es direkt vor einem Biegetest einer starken Säure ausgesetzt war. Ein mechanischer Test, der eine ausreichend lange Zeit nach der Säurebehandlung erfolgte, zeigte wieder duktiles Verhalten. Er führte diesen Effekt der vorübergehenden Versprödung auf im Metall gelösten Wasserstoff zurück. Mit dieser Entdeckung und Erklärung der Wasserstoffversprödung war der Grundstein für ein bis heute relevantes Forschungsgebiet gelegt. Das Verständnis der Vorgänge im Material ist schwierig, da elektrochemische Prozesse, Mikrostruktur und mechanische Eigenschaften involviert sind und sich gegenseitig beeinflussen. Des Weiteren hat Wasserstoff in Metallen eine meist hohe Mobilität, eine geringe Löslichkeit und ist mit Standardmethoden, wie zum Beispiel energiedispersiver Röntgenspektroskopie (EDX), nicht oder nur schwer detektierbar. Dadurch kann man in Messungen meist nur die Wirkung des Wasserstoffs auf das Material, zum Beispiel eine Herabsetzung der Duktilität in einem Zugversuch, nachweisen [Nag16].

In einer Vielzahl von Veröffentlichungen, die in den folgenden Review-Artikeln zusammengefasst sind $\left[\mathrm{RFM}^{+}\right.$09, Lyn12, Nag16], findet man eine detaillierte Darstellung der Grundlagen. Es wird Wasserstoffversprödung und eine daraus hervorgehende erleichterte Rissbildung beschrieben. Wichtige Grundlagen sind die Löslichkeit und Diffusion von Wasserstoff im Eisengitter und das Haften an Gitterdefekten. Zusätzlich sind in diesen Artikeln $\left[\mathrm{RFM}^{+}\right.$09, Lyn12, Nag16] die Mechanismen diskutiert, welche die Wirkung von Wasserstoff auf die mechanischen Eigenschaften erklären. Dabei sind zwei Mechanismen besonders hervorzuheben: Der hydrogen enhanced decohesion (HEDE) Mechanismus beschreibt, dass durch die Anwesenheit von Wasserstoff im Metallgitter die Bindungsstärke herabgesetzt ist und Metallbindungen daher leichter aufgebrochen werden können; Weiter beschreibt der hydrogen enhanced localised plasticity (HELP) Mechanismus, dass eine lokal erhöhte Plastizität durch Wasserstoff hervorgerufen werden kann. Folglich kommt es zu einer erhöhten Bildung und Bewegung von Versetzungen. Diese zunächst widersprüchlich erscheinenden Mechanismen werden in Kapitel 2 der vorliegenden Dissertation näher beschrieben und in Kapitel 6 werden sie auf Basis der in dieser Arbeit erlangten Ergebnisse diskutiert.

Über die Veränderung der mechanischen Eigenschaften hinaus, kann Wasserstoff auch ohne anliegende äußere Spannung Risse in Materialien hervorrufen [CS93, EMD $\left.{ }^{+} 11\right]$. Es 
muss dazu ein hohes chemisches Potential des Wasserstoffs vorliegen. Das Phänomen wird Wasserstoff-induzierte Rissbildung hydrogen induced cracking (HIC) genannt. Als Triebkraft für die Rissbildung wird ein hoher Wasserstoffdruck in den Rissen angegeben [RZC $\left.{ }^{+} 07\right]$, allerdings auch kontrovers diskutiert [Lan01]. Eine direkte Messung des Drucks in Rissen ist nicht möglich. Griesche et al. [GDK $\left.{ }^{+} 14\right]$ konnten den Druck in einzelnen Rissen aus Neutronenstreuexperimenten folgern. Sie geben einen Druck im Bereich von 20\% der Streckgrenze von Eisen an. Im Zusammenhang mit der Rissbildung im Inneren des Materials werden Materialaufwürfe auf der Oberfläche beobachtet [EMD $\left.{ }^{+} 11\right]$. Diese werden im Folgenden Blister genannt. Sie entstehen durch die Materialverschiebung, die von oberflächennahen Rissen ausgeht [EMD $\left.{ }^{+} 11\right]$.

Ebenso wie die Triebkraft der Rissbildung ist auch der Einfluss der Wasserstoffversprödung bei der Rissinitiierung und dem Rissfortschritt durch das Material noch nicht im Detail verstanden und daher Thema der vorliegenden Dissertation.

\section{\begin{tabular}{l|l} 
Fragestellungen der Arbeit & 1.3
\end{tabular}}

Ein bekanntes Phänomen ist, dass die Löslichkeit und die Verteilung des Wasserstoffs im Material stark von vorhandenen Gitterdefekten abhängt. Andererseits wird aber auch die Existenz und die Wechselwirkung der Gitterdefekte untereinander durch die Anwesenheit des Wasserstoffs beeinflusst. Dieses kann thermodynamisch durch das Defactants-Konzept beschrieben werden, vergleiche [Kir07a, Kir07b]. Ein Beispiel ist eine erhöhte Leerstellenkonzentration in Anwesenheit von Wasserstoff, sogenannte Superabundant Vacancies [Fuk03].

Bei einer sehr hohen Wasserstoffkonzentrationen kann es, wie im vorherigen Abschnitt beschrieben, zu einer Rissbildung im Material kommen, ohne dass eine äußere mechanische Spannung anliegt [CS93, EMD $\left.{ }^{+} 11\right]$. Das Material wird dann innerlich makroskopisch geschädigt und die Schädigung rührt ausschließlich vom Wasserstoff her. Deshalb eignet sich die Untersuchung dieses Phänomens der Rissbildung durch Wasserstoff besonders gut, um die Wirkung von Wasserstoff auf Materialien grundlegend zu untersuchen. In der vorliegenden Arbeit wurde daher HIC in reinem Eisen untersucht. Reines Eisen wurde als Modellsystem gewählt, da Eisen den Hauptbestandteil von Stahl, dem meistverwendeten metallischen Werkstoff, darstellt. Um ein möglichst einfaches System zu betrachten, wurde Eisen mit einer hohen Reinheit verwendet. Das Eisen wurde einer Wärmebehandlung unterzogen, daher liegt eine geringe Defektdichte vor.

Das Ziel der Arbeit ist, in diesem Modellsystem die Rissbildung durch Wasserstoff ohne 
äußere mechanische Belastung zu verstehen. Das wird erst durch die Wahl des Systems möglich, da sich sonst Effekte ${ }^{1}$ überlagern. Die detaillierte Untersuchung der Rissbildung gliedert sich in folgende Fragestellungen:

- An welchen Stellen im Material findet die Initiierung der Risse statt? Experimente mit verschiedenen Stählen und Eisen zeigen, dass Einschlüsse im Material, besonders länglich geformte Mangansulfide, typische Initiierungsstellen sind [BDK09, RCL $\left.{ }^{+} 08\right]$. Weiter wird eine Ansammlung von Leerstellen als Ausgangspunkt von Rissen vorgeschlagen $\left[\mathrm{RZC}^{+} 07, \mathrm{RCL}^{+} 08\right]$. In dieser Arbeit soll herausgefunden werden, ob Einschlüsse oder Leerstellencluster die Initiierungsstellen darstellen und welchen Effekt eine Änderung der Reinheit hervorruft.

- Wie kommt es zur Rissinitiierung?

Elektronenmikroskopische Untersuchungen und theoretische Überlegungen sollen Aufschluss darüber geben, welche Prozesse bei der Rissinitiierung ablaufen.

- Was ist die Triebkraft für die Bildung von Rissen?

Es wird oft ein sehr hoher Wasserstoffdruck als Triebkraft für die Rissbildung vorgeschlagen $\left[\mathrm{RZC}^{+} 07\right]$. Griesche et al. $\left[\mathrm{GDK}^{+} 14\right]$ konnte aus Neutronenstreuexperimenten den Druck in einzelnen Rissen abschätzen. Eine Druckmessung, welche über alle Risse mittelt, wurde in dieser Arbeit basierend auf [Leh14] weiterentwickelt. Der Druck in den Rissen soll so experimentell bestimmt werden.

- Wie geht der Rissfortschritt durch das Eisen vonstatten und welchen Einfluss hat die Wasserstoffversprödung?

Kristallographische Untersuchungen des umliegenden Materials um Risse sollen Aufschluss über Rissebenen geben. Zusätzlich wird die Defektstruktur mittels Transmissionselektronenmikroskopie (TEM) untersucht. Durch diese Ergebnisse kann der Einfluss der Wasserstoffversprödung auf Basis des HELP-Mechanismus diskutiert werden.

Auf Basis der detaillierten Untersuchungen dieser Teilaspekte ist es das Ziel der vorliegenden Dissertation, einen vollständigen Mechanismus der Rissbildung in reinem defektarmen Eisen durch Wasserstoffbeladung vorschlagen zu können.

\footnotetext{
${ }^{1}$ Zum Beispiel ist es schwierig, die Defektentstehung in einem Material zu untersuchen, in dem schon vor der Beladung verschiedene Defekte vorhanden sind.
} 


\section{Theorie und Hintergrund 2}

In diesem Kapitel werden die theoretischen Grundlagen vorgestellt, die zum Verständnis und zur Interpretation der Ergebnisse dieser Arbeit beitragen sollen. In Abschnitt 2.1 werden Grundlagen zur Löslichkeit, Diffusion und Lokalisation von Wasserstoff im EisenGitter und an Defekten beschrieben.

Darauf folgt in Abschnitt 2.2 eine Beschreibung der plastischen Verformung und von Brüchen in Metallen. In dem sich anschließenden Abschnitt 2.3 wird vorgestellt, wie sich die Verformung in Gegenwart von Wasserstoff verändert. Dabei wird auf verschiedene Mechanismen, die zur Wasserstoffversprödung in Eisen führen können, näher eingegangen.

\section{\begin{tabular}{l|l} 
Wasserstoff in Eisen & 2.1
\end{tabular}}

\section{\begin{tabular}{l|l} 
Löslichkeit von Wasserstoff in Eisen & 2.1 .1
\end{tabular}}

In Eisen löst sich bei Raumtemperatur nur wenig Wasserstoff, siehe Abbildung 2.1, daher gibt es wenig experimentelle Daten für die Löslichkeit bei dieser Temperatur. Hirth [Hir80] leitete aus den Daten von Quick und Johnson [QJ78] folgenden Zusammenhang für den Wasserstoffgehalt $\theta[a t . \%]$ ab :

$$
\theta=0,00185 \cdot \sqrt{\frac{p}{0,1 \mathrm{MPa}}} \cdot \exp \left(-\frac{3440 \mathrm{~K}}{T}\right),
$$

wobei $p$ der Wasserstoffdruck und $T$ die Temperatur ist. In dieser Gleichung findet sich das bekannte Gleichgewicht zwischen Wasserstoffkonzentration im Metallgitter und Wasserstoffgasdruck $\theta \propto \sqrt{p}$ aus dem Sievertsschen Gesetz wieder [Sie29].

Die endotherme Lösungsenthalpie kann aus dieser Temperaturabhängigkeit berechnet werden. Sie beträgt $\Delta H=28,6 \mathrm{~kJ} /(\mathrm{Mol} \mathrm{H})$. Zusätzlich kann die Gleichgewichtskonzentration von Wasserstoff in Eisen bei Raumtemperatur (RT) extrapoliert werden. Sie beträgt

$$
\theta(\mathrm{RT})=2 \times 10^{-8} \text { at. } \%
$$

bei einem Druck von 0,1 MPa. In Messungen bei Raumtemperatur werden oft Wasserstoffkonzentrationen in der Größenordnung von $1 \mu \mathrm{g} / \mathrm{g}$ nachgewiesen (entspricht in etwa $56 \times 10^{-4}$ at.\%), sodass der Wasserstoff größtenteils an Defekten getrappt ist [Nag16]. 


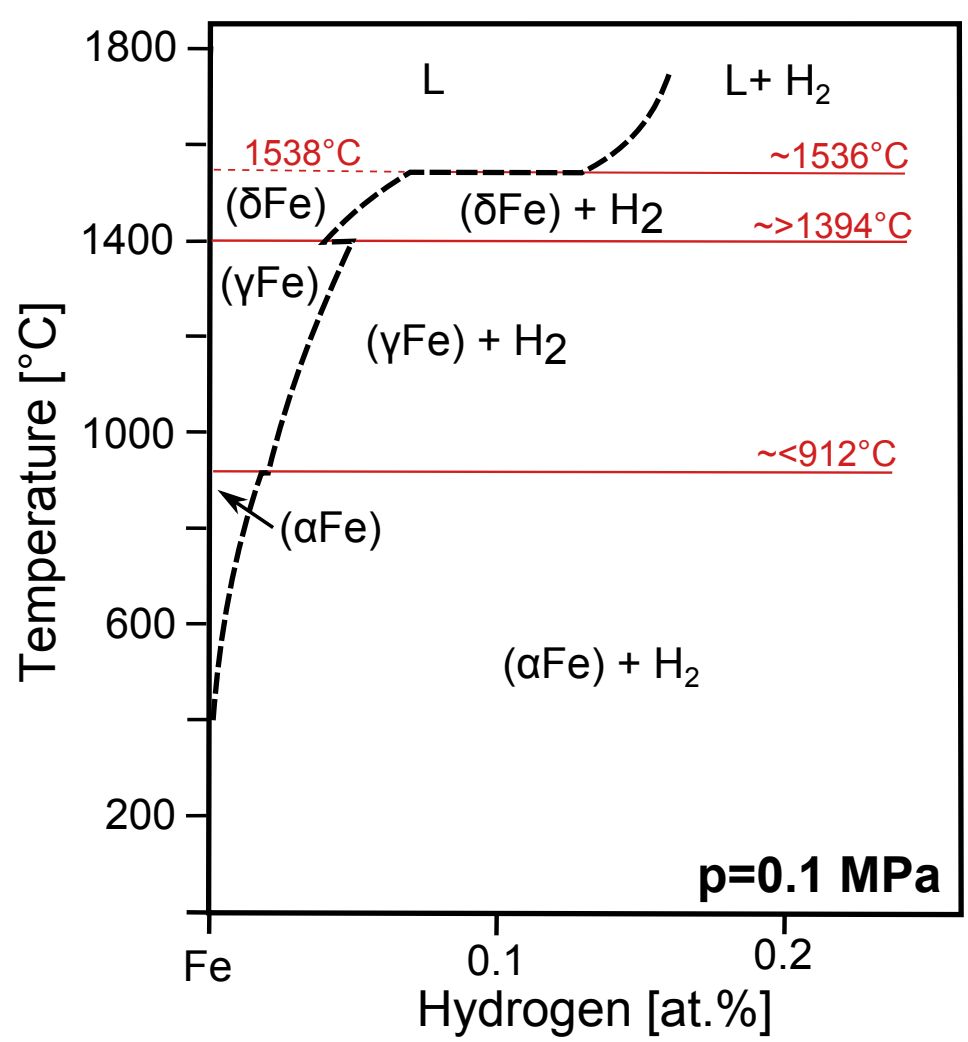

Abbildung 2.1: Nach Abbildung 1 in [SMM90]. Phasendiagramm von Eisen und Wasserstoff bei einem Druck von $0,1 \mathrm{MPa}$ Wasserstoff. $\alpha$ - und $\delta$-Eisen sind kubischraumzentriert und $\gamma$-Eisen ist kubisch-flächenzentriert.
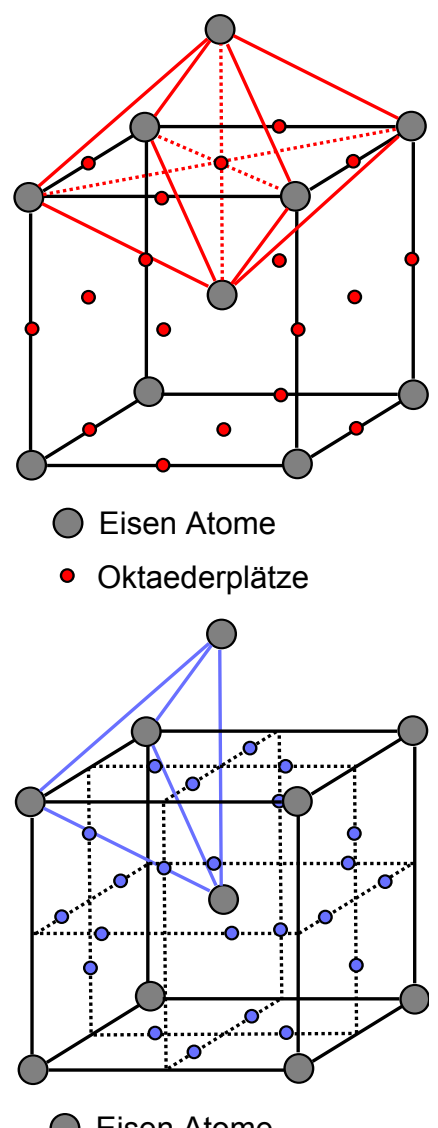

Eisen Atome

- Tetraederplätze

Abbildung 2.2: Nach Abbildung 1 in [JC04]. Skizze zur Veranschaulichung von Oktaeder- und Tetraeder-Zwischengitterplätzen in krz Materialien, hier Eisen.

\section{Position von Wasserstoff im Eisengitter}

Wasserstoff befindet sich generell auf Zwischengitterplätzen. In Abbildung 2.2 sind Tetraeder- und Oktaederplätze im Eisengitter skizziert. Wasserstoff befindet sich bevorzugt auf Tetraederplätzen [JC04, Wip97]. Bei sehr hohen Wasserstoffkonzentrationen ist laut theoretischen Berechnungen ein Aufenthalt auf Oktaederplätzen energetisch günstiger und es kommt zu einer tetragonalen Verzerrung des krz Gitters in ein tetragonal-raumzentriertes Gitter [SFAdA08]. 


\section{\begin{tabular}{l|l} 
Diffusion von Wasserstoff in Eisen & 2.1 .3
\end{tabular}}

Dieser Abschnitt orientiert sich an den Ausführungen von Wipf [Wip01]. In krz Materialien sind die Diffusionskoeffizienten von Wasserstoff generell hoch im Vergleich zu kubisch-flächenzentrierten (kfz). Dieses ist durch einen vergleichsweise kleinen Abstand der relevanten Zwischengitterplätze zu erklären (Tetraederplätze in krz- im Vergleich zu Oktaederplätze in kfz-Materialien). Der Abstand in krz-Materialien ist so klein, dass die Wasserstoffatome zwischen den Tetraederplätzen tunneln können, was vor allem bei niedrigen Temperaturen zu hohen Diffusionskoeffizienten führt [FS85]. Verschiedene Messungen des Diffusionskoeffizienten von Eisen variieren über mehrere Größenordnungen, zum Beispiel [BBMN66]. Der Grund ist, dass der Reinheits- und Verformungsgrad der Probe einen erheblichen Einfluss auf die Diffusionsgeschwindigkeit hat. Ein Diffusionskoeffizient für defektarmes Eisen mit einer Reinheit von 99,99\% wurde in [MF63] bestimmt und beträgt:

$$
D\left[\mathrm{~m}^{2} / \mathrm{s}\right]=5,8 \times 10^{-8} \exp \left(\frac{-4,5[\mathrm{~kJ} / \mathrm{Mol}]}{\mathrm{RT}}\right),
$$

das entspricht einer Diffusionskonstanten von $10^{-8} \mathrm{~m}^{2} / \mathrm{s}$ bei Raumtemperatur. Diese Größenordnung wurde auch von Grabke et al. [GR00] gemessen.

\section{\begin{tabular}{l|l} 
Wechselwirkung von Wasserstoff mit Gitterdefekten & 2.1 .4
\end{tabular}}

Wasserstoff kann in Eisen nicht nur interstitiell gelöst vorliegen, sondern wird auch an verschiedenen Gitterdefekten getrappt. In Abbildung 2.1.4 ist schematisch gezeigt, wo der Wasserstoff lokalisiert sein kann. Durch die Ansammlung von Wasserstoff an Defekten kann die Gesamtenergie des Systems im Vergleich zur Lösung des Wasserstoffs auf Zwischengitterplätzen abgesenkt werden. Zudem wird die Bildung neuer Defekte erleichtert, was thermodynamisch durch das Defactants Konzept [Kir07a, Kir07b] beschrieben wird. Es beschreibt die Änderung der Bildungsenergie eines Defektes $\gamma$ durch den Überschussgehalt $\Gamma_{H}$ von Wasserstoff an einem Defekt. $\mu_{H}$ ist das chemische Potential vom Wasserstoff, welches mit der Konzentration von gelöstem Wasserstoff in Eisen steigt:

$$
\mathrm{d} \gamma=\Gamma_{H} \mathrm{~d} \mu_{H}
$$

Diese Gleichung ist eine Verallgemeinerung der Gibbs-Adsorptionsisotherme, welche die Herabsetzung der Oberflächenspannung in einer Flüssigkeit durch Tenside beschreibt [Gib28]. 


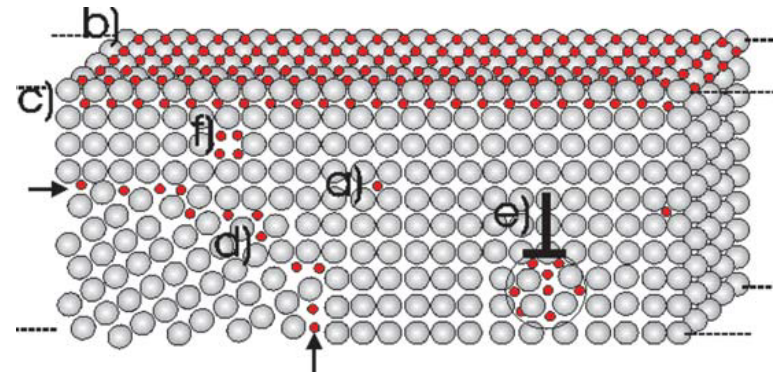

Abbildung 2.3: Reprint aus [Pun04], Copyright 2004, mit Genehmigung von John Wiley and Sons. Schematische Darstellung der Traps von Wasserstoff im Eisengitter (a) und an verschiedenen Gitterdefekten: b) an der Oberfläche, c) unter der Oberfläche, d) an Korngrenzen, e) im Spannungsfeld von Versetzungen, f) in Leerstellen.

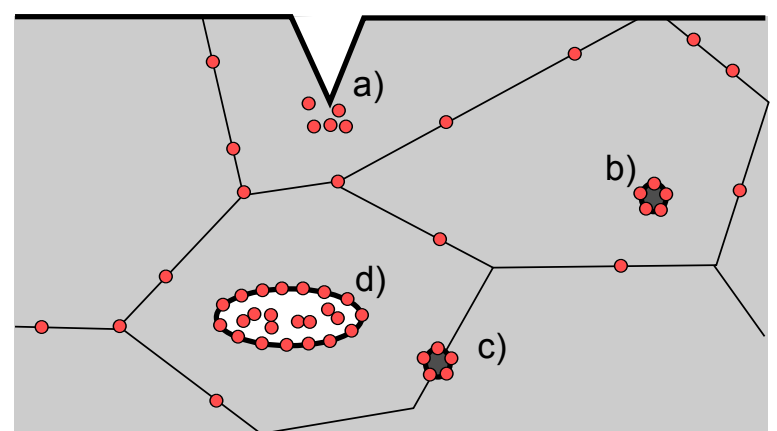

Abbildung 2.4: Nach Abbildung 1B in [Lyn12]. Schematische Darstellung von Traps an makroskopischen Defekten: a) vor einer Rissspitze, b) an der Grenzfläche zu einem Einschluss c) an der Grenzfläche zu einem Einschluss, der an einer Korngrenze lokalisiert ist, d) an der Oberfläche eines Hohlraums und gasförmig in dessen Innenraum.

Mit Gleichung (2.4) kann zum Beispiel eine deutlich erhöhte Leerstellenkonzentration in Gegenwart von Wasserstoff erklärt werden (Superabundant Vacancies) [Fuk03]. Ebenso ist die Bildungsenergie von Versetzungen herabgesetzt [BV10], siehe auch Abschnitt 2.3.3. Die Erhöhung der Defektdichte beziehungsweise die Herabsetzung der Energie diese Defekte zu bilden, kann einen erheblichen Einfluss auf die Rissbildung und den Rissfortschritt haben. Dieses wird später in Abschnitt 6 diskutiert.

Wasserstoff wird, wie in Abbildung 2.4 schematisch gezeigt, auch an makroskopischen Defekten getrappt, dazu werden in diesem Fall Einschlüsse im Material und Hohlräume gezählt. Ein Vergleich der Aktivierungsenergien verschiedener Wasserstofftraps ist Tabelle $2.1 \mathrm{zu}$ entnehmen. Diese wurden mittels thermischer Analyse von elektrochemisch beladenem Eisen ermittelt. Man kann der Tabelle entnehmen, dass besonders an den Grenzflächen zu nichtmetallischen Einschlüssen Wasserstoff stark getrappt wird. Daraus resultiert bei Wasserstoffbeladung eine hohe Konzentration an der Grenzfläche.

\section{\begin{tabular}{l|l} 
Verformung und Bruch & 2.2
\end{tabular}}

\section{Verformung in krz Materialien $\mid 2.2 .1$}

Dieser Abschnitt orientiert sich an [Got01, CR12]. Bei der Verformung von einkristallinen Materialien werden auf der Oberfläche des Materials nach der Verformung Gleitstufen 


\begin{tabular}{l|c} 
Trap & Aktivierungsenergie $[\mathrm{kJ} / \mathrm{Mol}]$ \\
\hline Korngrenze & 16,30 und 18,20 \\
Versetzung & 26,04 \\
Microvoid in kaltgewalztem Eisen & 35,15 und 40,31 \\
Microvoid in AISI 4340 Stahl & 56,20 \\
Grenzfläche zu Eisenoxid & 47,20 \\
Grenzfläche zu MnS & 72,30 \\
Grenzfläche zu A1 $1_{2} 0_{3}$ & 78,96 \\
Grenzfläche zu TiC & 86,98
\end{tabular}

Tabelle 2.1: Entnommen aus [LL86] und darin enthaltenen Referenzen. Übersicht der Aktivierungsenergien verschiedener Wasserstoff-Traps (doppelte Werte aus unterschiedlichen Quellen). Sie wurden mittels thermischer Analyse ermittelt. Besonders tiefe Traps stellen Grenzflächen zu nicht-metallischen Einschlüssen dar.

beobachtet. Diese entstehen, wenn die Verformung des Materials durch Abgleiten entlang kristallographischer Ebenen erfolgt. Mikroskopisch erfolgt die Abgleitung über das Durchlaufen einer Versetzung, vergleiche Abbildung 2.5
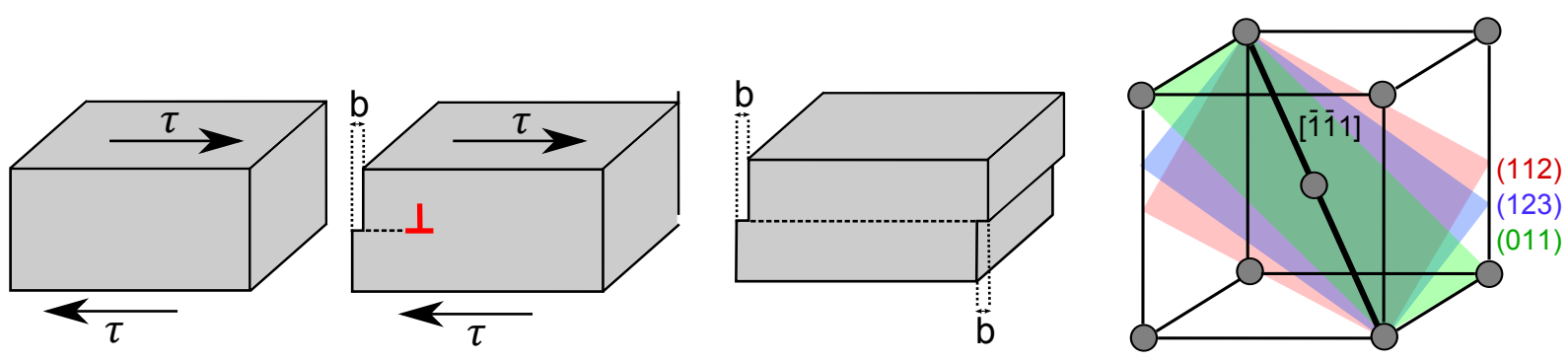

Abbildung 2.5: Nach Abbildung 6.17 in [Got01]. Skizze zur Veranschaulichung der plastischen Verformung eines Einkristalls, die mit der Bildung von Gleitstufen einhergeht. $\tau$ bezeichnet die von außen angelegte Schubspannung und $b$ den Betrag eines Burgers-Vektor.

Abbildung 2.6: Nach Abbildung 6.21 in [Got01]. Darstellung der Gleitebenen im krz Kristall. Die Gleitebenen enthalten alle die $\langle 111\rangle$ Gleitrichtung.

Die Schubspannung, die auf eine Gleitebene wirken muss, damit sich eine Versetzung bewegen kann, wird Peierls-Spannung $\tau_{p}$ genannt und ist gegeben durch

$$
\tau_{p}=\frac{2 G}{1-\nu} \exp \left(-\frac{2 \pi}{1-\nu} \frac{d}{b}\right)
$$

In dieser Gleichung sind das Schubmodul $G$ und die Querkontraktionszahl $\nu$ Materialkonstanten. $d$ ist der Gitterebenenabstand und $b$ der Betrag des Burgers-Vektor. Die Peierls- 
Spannung hängt demnach exponentiell von $d / b$ ab. Daher sind Gleitebenen niedrigindizierte Ebenen, da diese einen großen Ebenenabstand $d$ haben. Gleitrichtungen zeichnen sich durch einen kleinen Burgers-Vektor aus. In krz Materialien sind die $\{110\}$ Ebenen am dichtesten gepackt, allerdings ist der Unterschied zu $\{112\}$ und $\{123\}$ Ebenen nicht groß, sodass diese drei Ebenenscharen als Gleitebenen beobachtet werden. Die Gleitrichtung ist $\langle 111\rangle$, vergleiche Abbildung 2.6.

Die tatsächlich aufzubringende Schubspannung, kritische Schubspannung genannt, bei der eine Verformung eines Einkristalls einsetzt, hängt zusätzlich zur Peierls-Spannung von der Orientierung der Probe zu der angelegten Spannung ab. Entscheidend ist nur die Kraft, die in der Gleitebene in Richtung des Burgers-Vektors wirkt. Die auf die Versetzung wirkende Zugspannung $\tau$ in Abhängigkeit der angelegten Zugspannung $\sigma$ wird durch die Peach-Koehler-Gleichung beschrieben:

$$
\tau=\cos \kappa \cdot \cos \lambda \cdot \sigma=m \cdot \sigma
$$

wobei $\kappa$ der Winkel zwischen Zugrichtung und Gleitebenennormale und $\lambda$ der Winkel zwischen Zugrichtung und Gleitrichtung ist. $m=\cos \kappa \cdot \cos \lambda$ wird Schmid-Faktor genannt und beschreibt die Lage eines Gleitsystems relativ zur angelegten Spannung. Gibt es mehrere Gleitsysteme, wird das Abgleiten entlang des Systems mit dem höchsten Schmid-Faktor zuerst stattfinden.

Das Schmidsche Schubspannungsgesetz beschreibt weiter, dass die kritische Schubspannung $\tau_{0}$, die für die Versetzungsbewegung überwunden werden muss, für alle Gleitsysteme gleich ist. Die kritische Schubspannung ist entscheidend für das Einsetzen von plastischer Verformung eines Kristalls. In krz Materialien ist in der Regel $\tau_{0}<\tau_{p}$. Das bedeutet, dass die PeierlsSpannung, siehe Gleichung 2.5, ausschlaggebend für die kritische Zugspannung ist. Sie ist durch thermische Aktivierung kleiner als $\tau_{p}$. Es gibt auch den Fall $\tau_{0}>\tau_{p}$. Dieser tritt bei kfz Materialien auf, und soll daher hier nicht näher betrachtet werden. Die Streckgrenze $R_{e}$ des Materials bezeichnet den Betrag der Spannung, bei dem plastische Verformung einsetzt, und kann aus der kritischen Schubspannung berechnet werden:

$$
R_{e}=\frac{\tau_{0}}{(\cos \kappa \cdot \cos \lambda)_{\max }} .
$$

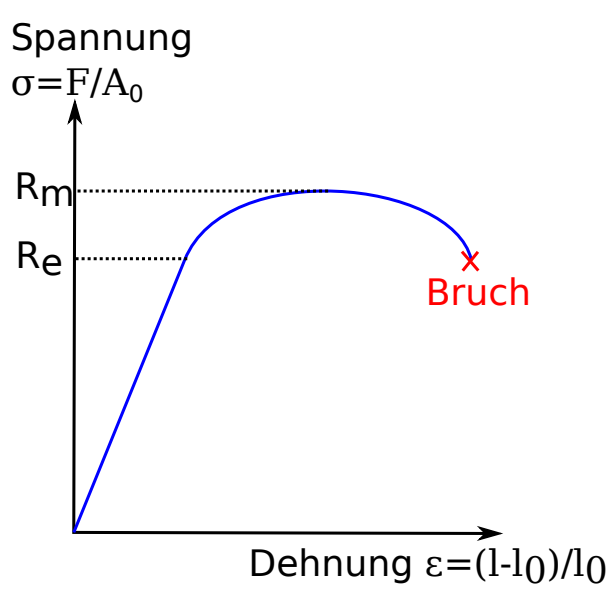

Abbildung 2.7: Skizze zur Veranschaulichung der Streckgrenze $R_{e}$, die den Übergang vom elastischen in den plastischen Bereich darstellt, und Zugfestigkeit $R_{m}$, die die maximale Spannung im Diagramm angibt. $F$ ist die Zugkraft und $A_{0}$ die Querschnittsfläche der Probe. 
$R_{e}$ ist für $\kappa$ und $\lambda=45^{\circ}$ maximal und beträgt $R_{e}^{\max }=2 \tau_{0}$.

In einem Zugversuch kann die Streckgrenze experimentell bestimmt werden. In Abbildung 2.7 ist ein typisches Spannungs-Dehnungs-Diagramm eines Zugversuches skizziert. Zunächst ist ein linearer Bereich zu sehen. Hier findet eine elastische Verformung der Probe statt, die bei Entlastung reversibel ist. Danach folgt die plastische Verformung, die mit Versetzungsbewegung einhergeht. Diese Verformung ist irreversibel. Die Streckgrenze $R_{e}$ ist die Spannung, bei welcher das linear elastische Verhalten endet. Oft ist dieser Punkt schwer abzulesen, da der Übergang kontinuierlich ist. Zur Ermittlung wird oft der Schnittpunkt der extrapolierten Geraden an den elastischen Bereich und den Anfang des plastischen Bereiches verwendet. Alternativ wird häufig auch $R_{e 0,2}$ angegeben, dieser Wert beschreibt den Spannungswert, bei welchem nach Entlastung eine Dehnung von $0,2 \%$ bestehen bleiben würde.

\section{\begin{tabular}{l|l} 
Spröder und duktiler Bruch & 2.2 .2
\end{tabular}}

Die Ausführungen in diesem Abschnitt orientieren sich an [CR12]. Mechanisches Verhalten wird oft in spröde oder duktil unterschieden. Daher wird in diesem Abschnitt die Duktilität genauer definiert. Außerdem werden typische Merkmale von sprödem beziehungsweise duktilem Bruch eingeführt.

Ein Material wird als spröde bezeichnet, wenn es sich zum Beispiel in einem Zugversuch gar nicht beziehungsweise nur sehr wenig plastisch verformt, bevor es bricht. Daraus resultiert, dass (nahezu) keine Einschnürung oder Dehnung des Materials vorliegt. Diese beiden Merkmale können zur Quantifizierung der Duktilität genutzt werden. Die Bruchdehnung $\varepsilon_{B}$ ist definiert als Längenänderung der Probe nach dem Bruch bezogen auf die Ausgangslänge:

$$
\varepsilon_{B}=\left(\frac{l_{\text {end }}-l_{0}}{l_{0}}\right)
$$

wobei $l_{\text {end }}$ die Länge der Probe nach dem Zugversuch ist, beziehungsweise die Summe der Längen der Bruchstücke, und $l_{0}$ die Ausgangslänge der Probe vor dem Zugversuch. Alternativ kann die Brucheinschnürung angegeben werden. Man spricht von einem spröden Material, wenn die Bruchdehnung weniger als $5 \%$ beträgt [CR12].

Um zu untersuchen, ob ein Bruchprozess duktil oder spröde ist, kann nicht immer ein Zugversuch durchgeführt werden. Oft interessiert zum Beispiel nach dem Bruch eines Bauteils in der Anwendung, welches Bruchverhalten vorlag. Es muss dann anhand der Bruchfläche und des umliegenden Materials auf das Bruchverhalten geschlossen werden. Hierbei sind auf den Bruchflächen von duktilen oder spröden Brüchen Merkmale zu beobachten, die auf den Bruchprozess zurückschließen lassen. Diese sollen im Folgenden eingeführt werden. 


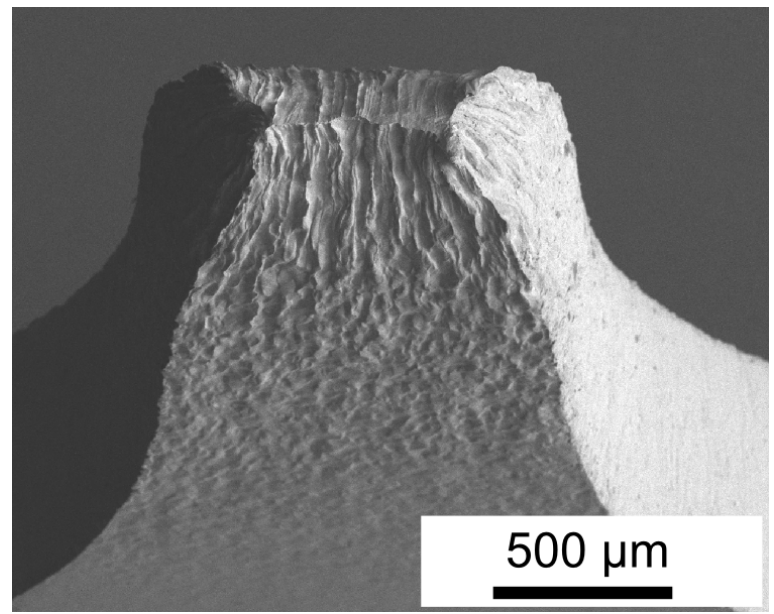

Abbildung 2.8: Bild erstellt in Zusammenarbeit mit Jonas Wawra [Waw16]. Rasterelektronenmikroskop (REM)-Bild einer TantalProbe nach einem Zugversuch. Eine Einschnürung von fast $100 \%$ ist zu sehen. Man spricht von der Entstehung einer „Messerkante“.

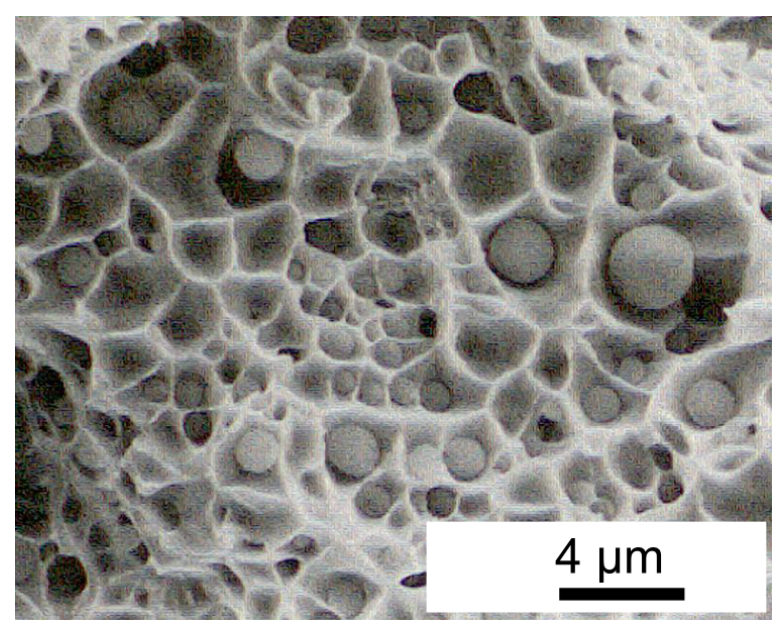

Abbildung 2.10: Reprint aus [Lan01], Copyright 2014, mit Genehmigung von John Wiley and Sons. REM-Bild, das einen duktilen Bruch von ferritischem Stahl zeigt. In den Mulden sind Mangansulfid-Einschlüsse zu sehen.

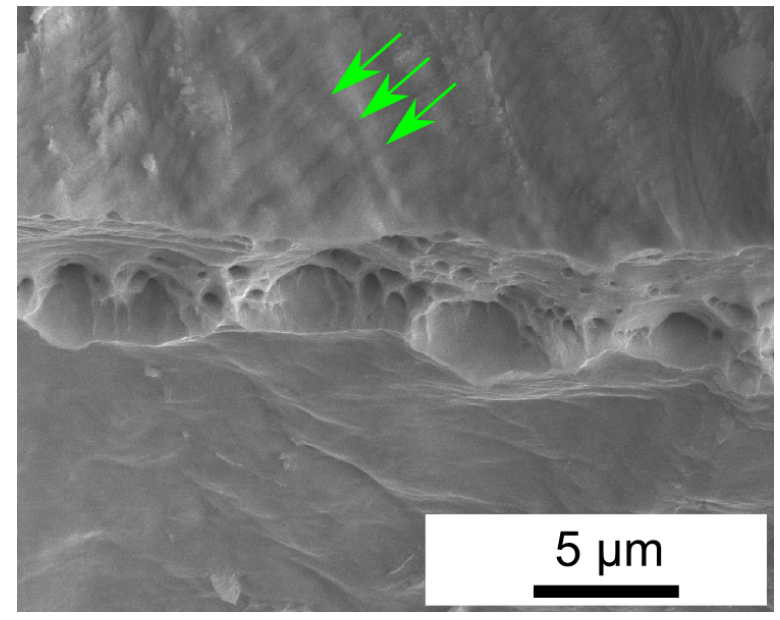

Abbildung 2.9: Bild erstellt in Zusammenarbeit mit Jonas Wawra [Waw16]. Vergrößerung von Abbildung 2.8. Die Bruchkante ist ca. $3-5 \mu \mathrm{m}$ breit und zeigt Mulden, die durch Microvoid-Coalescence verursacht werden. Oberhalb der Bruchkante sind Gleitstufen zu erkennen, die exemplarisch mit Pfeilen markiert sind.
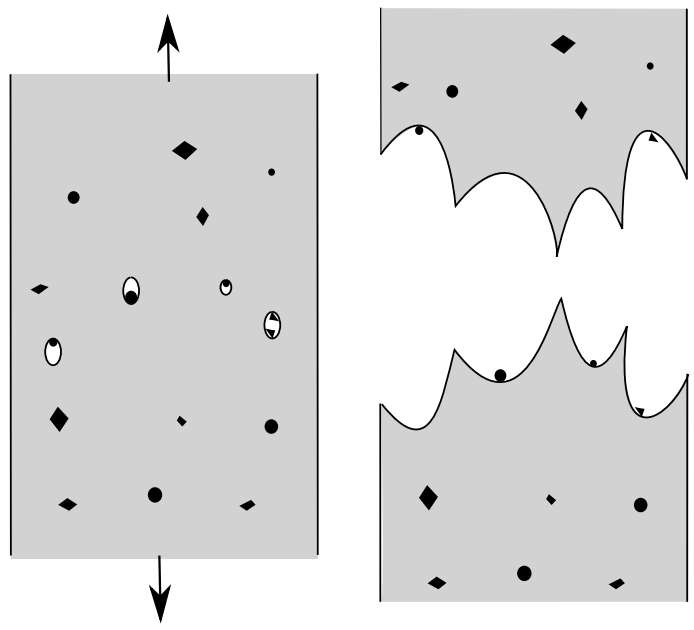

Abbildung 2.11: Nach Abbildung 5.9 in [Lan01]. Skizze zur Veranschaulichung, wie Mulden bei duktilem Bruch entstehen. Am Rand der Mulden sind häufig Gleitstufen zu finden. 


\section{Der duktile Gleitbruch}

Dieser Abschnitt orientiert sich an [Sta97, Lan01]. Der zugrunde liegende Mechanismus eines Gleitbruchs ist das Abgleiten kristallographischer Ebenen, wie es zuvor in Abschnitt 2.2.1 beschrieben wurde. Dabei findet in der Regel eine große makroskopische Verformung statt. Bei reinen Metallen kann eine Einschnürung von bis zu 100\% auftreten. Ein Beispiel aus der Bachelorarbeit von Jonas Wawra [Waw16] ist in Abbildung 2.8 zu sehen. Es handelt sich um reines Tantal, an dem ein Zugversuch bis zum Bruch durchgeführt wurde. Die Probe hat sich während des Zugversuchs stark plastisch verformt, sodass die Bruchkante weniger als $5 \mu \mathrm{m}$ breit ist, wobei die Probendicke ursprünglich $1 \mathrm{~mm}$ betrug. Es lag kurz vor dem Bruch eine Einschnürung von fast 100\% vor. Eine Detailaufnahme der Risskante, siehe Abbildung 2.9, zeigt Gleitstufen oberhalb der Bruchkante und kleine Mulden auf der Bruchkante. Gleitstufen entstehen bei der plastischen Verformung durch Abgleiten kristallographischer Ebenen, vergleiche dazu Abschnitt 2.2.1. In einem weiteren Beispiel von ferritischem Stahl, siehe Abbildung 2.10, sind diese Mulden über den gesamten Querschnitt der Probe zu sehen und sind ebenfalls charakteristisch für einen duktilen Bruch. Diese Mulden werden beobachtet, wenn in einem Material Hohlräume oder Einschlüsse vorhanden sind. Dann findet die plastische Verformung um diese Einschlüsse statt und es entsteht eine Bruchfläche aus Mulden. Der Mechanismus wird als Microvoid-Coalescence bezeichnet. Die Mulden sind tiefer, je duktiler das Material ist [Sta97]. Die Entstehung der Mulden ist schematisch in Abbildung 2.11 skizziert. Das Material verformt sich duktil um die meist nichtmetallischen und daher härteren Einschlüsse. Teilweise brechen die Einschlüsse spröde. In den Mulden sind die Einschlüsse wiederzufinden und dazwischen befindet sich stark verformtes Material, auf dem häufig Gleitstufen zu beobachten sind.

\section{Der spröde Spaltbruch}

Dieser Abschnitt orientiert sich an [Lan01, Sta97]. Bei einem Spaltbruch liegt ein anderer Mechanismus zugrunde, der zur Trennung des Materials führt. Ist eine kritische Normalspannung überschritten, können die Kohäsionskräfte innerhalb des Materials überwunden werden und es findet ein Bruch entlang einer bestimmten kristallographischen Ebene statt. Diese Ebene wird Spaltebene genannt. Spaltbrüche treten typischerweise in krz, aber nicht in kfz Materialien auf, da hier die kritische Schubspannung immer niedriger ist als die kritische Normalspannung und somit Abgleiten von Ebenen energetisch günstiger ist.

Spaltebenen sind immer niedrigindizierte Ebenen, da diese den größten Abstand zueinander haben. In Eisen sind die $\{100\}$-Ebenen Spaltebenen [Tap13]. In den Körnern eines polykristallinen Materials liegen die Spaltebenen unterschiedlich orientiert, sodass der Bruch plane Flächen innerhalb der Körner aufweist, siehe Abbildung 2.12. Auf den planen 


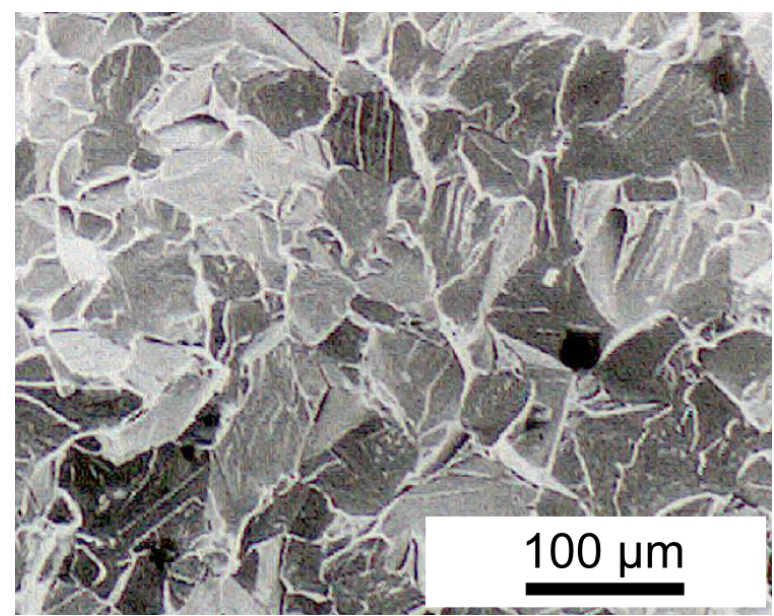

Abbildung 2.12: Reprint aus [Lan01], Copyright 2014, mit Genehmigung von John Wiley and Sons. REM-Aufnahme einer spröde gebrochenen Stahlprobe. Prüftemperatur lag bei $-196^{\circ} \mathrm{C}$

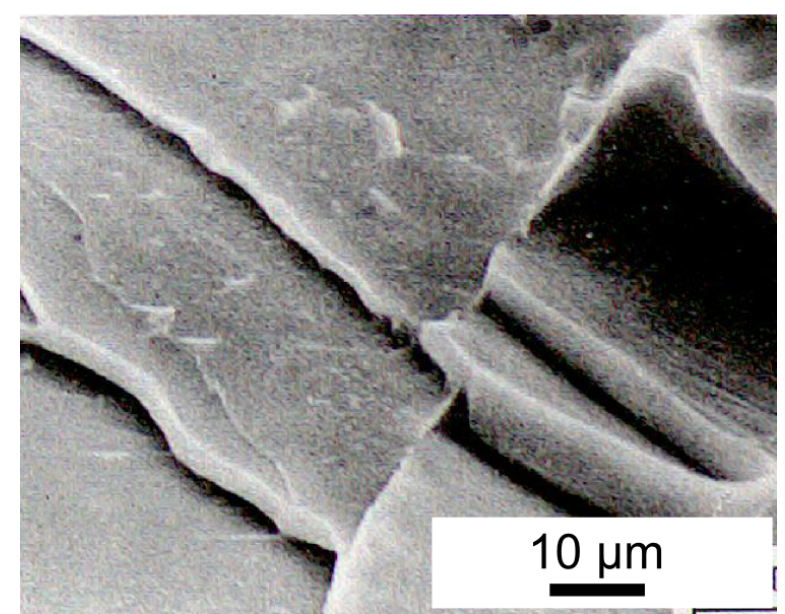

Abbildung 2.13: Reprint aus [Lan01], Copyright 2014, mit Genehmigung von John Wiley and Sons. REM-Aufnahme eines Spaltbruchs mit Stufen, welche über eine Korngrenze verlaufen.

Flächen werden häufig Stufen, die teilweise parallel verlaufen, beobachtet. Sie entstehen, wenn zwei Risse zusammenlaufen, eine Korngrenze überschritten wird oder Schraubenversetzungen auf der Spaltebene enden [PBP16]. Initiierungspunkte von Spaltrissen können sich schneidende Versetzungen, ein Aufstau von Versetzungen an einer Korngrenze oder einer Ausscheidung sein. Es ist zu bemerken, dass einem Spaltbruch leichte plastische Verformung vorausgehen kann.

Der Riss eines Spaltbruch kann auch entlang der Korngrenzen verlaufen. In diesem Fall spricht man von einem interkristallinen Bruch, der hier nicht näher behandelt werden soll.

\section{\begin{tabular}{l|l} 
Linear elastische Bruchmechanik & 2.2 .3
\end{tabular}}

Dieses Kapitel orientiert sich an den Ausführungen in [CR12, RHB06]. Die Theorie der Bruchmechanik beschreibt, wann es zu einem Rissfortschritt kommt, wenn im Material Risse oder Poren vorhanden sind. Sind solche Fehlstellen vorhanden, ist das mechanische Bruchverhalten nicht mehr allein durch die Streckgrenze und die Zugfestigkeit beschreibbar. Es wird linear-elastische Bruchmechanik verwendet, das heißt, die Ausführungen sind generell für spröde Materialien gültig. Allerdings sind sie auch auf duktile Materialien anwendbar, wenn die plastische Verformung auf ein Gebiet nahe der Rissspitze beschränkt ist.

Der Spannungsintensitätsfaktor $K_{t}$ beschreibt das Verhältnis von der maximalen Spannung, die in einem Material mit einem Riss auftritt, zu der anliegenden Spannung. Er 
wurde von Irwin [Irw57] eingeführt und ist aus einer Vereinfachung der Herleitung von Westergaard [Wes39], die eine Beschreibung des Spannungszustandes um einen Riss darstellt, abgeleitet. Betrachtet man als Näherung eines Risses ein elliptisches Loch in einem Blech, an dem senkrecht dazu eine Normalspannung anliegt, kann die Spannung $\sigma_{m}$ direkt an der Rissspitze durch folgenden Ausdruck näherungsweise beschrieben werden:

$$
\sigma_{m}=2 \sigma_{0} \sqrt{\frac{a}{\rho_{t}}} .
$$

Dabei ist $\sigma_{0}$ die anliegende Zugspannung, $\rho_{t}$ der Krümmungsradius der Rissspitze und $2 a$ die Länge des Risses. Der Spannungsintensitätsfaktor $K_{t}$ ist nun definiert als Verhältnis von $\sigma_{m} / \sigma_{0}$ und er beschreibt das Maß der Intensivierung der Spannung direkt vor der Rissspitze:

$$
K_{t}=\frac{\sigma_{m}}{\sigma_{0}}=\sqrt{\frac{a}{\rho_{t}}}
$$

\section{Wasserstoffversprödung $\mid 2.3$}

Dieser Abschnitt orientiert sich an $\left[\mathrm{RFM}^{+}\right.$09]. Die mechanischen Eigenschaften von Metallen können durch gelösten Wasserstoff stark beeinflusst werden. Es tritt häufig ein Übergang von duktilem zu sprödem Verhalten auf. Besonders eindrucksvoll kann dieser Effekt

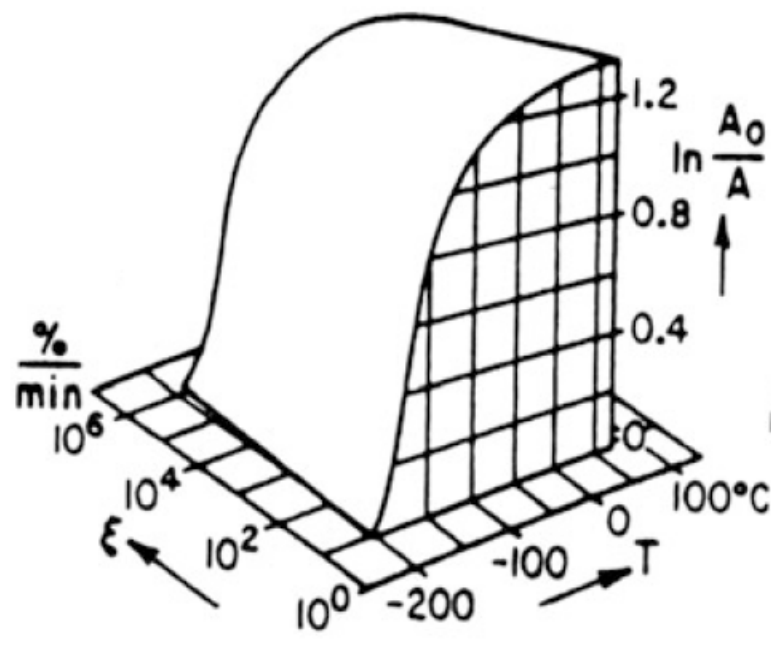

ohne Wasserstoff

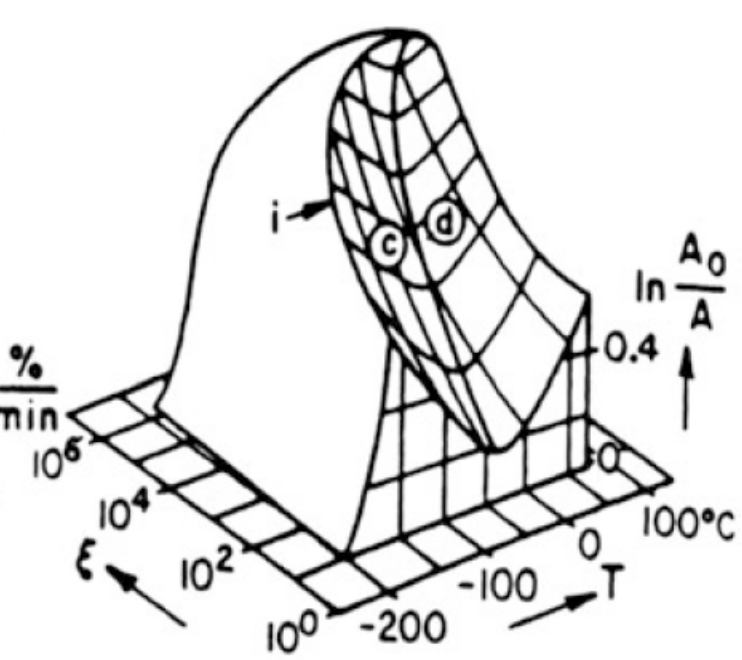

mit Wasserstoff

Abbildung 2.14: Reprint aus [Ber94], Copyright 2013, mit Genehmigung von John Wiley and Sons. Duktilität, angegeben in $\ln \frac{A_{0}}{A}$, von Baustahl als Funktion der Dehnrate und Temperatur mit und ohne Wasserstoffbeladung. Man erkennt eine starke Versprödung des Stahls durch Wasserstoff. Bei Raumtemperatur ist dieser Effekt sehr stark. 
in Zugversuchen eines Metalls in Gegenwart von Wasserstoff gezeigt werden. Ein Beispiel von Bernstein [Ber94] ist in Abbildung 2.14 gezeigt. Man sieht im linken Teil der Abbildung, dass die Duktilität, dargestellt als $\ln A_{0} / A$, ohne vorherige Wasserstoffbeladung mit der Temperatur zunimmt und nicht von der Dehnrate abhängt. Ist nun Wasserstoff in dem Material gelöst, verändert sich die Duktilität in Abhängigkeit der Temperatur vor allem für kleine Dehnraten, denn bei diesen hat der Wasserstoff mehr Zeit zur Rissspitze zu diffundieren und der Effekt ist größer als bei höheren Dehnraten. Die Duktilität steigt zunächst mit der Temperatur an, durchschreitet dann aber ein Minimum. Bei Raumtemperatur ist die Duktilität wesentlich niedriger als im Vergleichsexperiment, in dem kein Wasserstoff im Material gelöst war. Allerdings ist der zugrunde liegende Mechanismus dieser Versprödung Gegenstand aktueller Forschung und eine Fragestellung dieser Arbeit. Bei einer Untersuchung der Bruchflächen wird oft der sogenannte Quasi-Spaltbruch beobachtet zum Beispiel in [Bea72, $\mathrm{MFL}^{+} 11, \mathrm{MKT}^{+}$16]. Der Quasi-Spaltbruch verläuft im Gegensatz zu einem normalen Spaltbruch nicht entlang einer Spaltebene [RSN $\left.{ }^{+} 15\right]$. Auf den Bruchflächen werden oft Linien, sogenannte River Pattern in Richtung der Rissausbreitung beobachtet, siehe Abbildung 2.15. In Martin et al. [MFL $\left.{ }^{+} 11\right]$ wird ein Mechanismus vorgestellt, der die Entstehung dieser River Pattern erklärt. An Schnittpunkten von Gleitbändern entstehen Hohlräume, die ähnlich wie bei Microvoid-Coalescence wachsen, bis sie sich treffen und der finale Bruch stattfindet. Dieser finale Bruch ist duktil und die feinen weißen Linien auf der Bruchfläche entstehen. Die zugrunde liegende hohe Duktilität der Probe wird im HELP Mechanismus erklärt, vergleiche Abschnitt 2.3.3. Allgemein werden verschiedene Mechanismen diskutiert, die die Versprödung von Materialien durch Wasserstoff mikroskopisch erklären sollen. Die wichtigsten werden in den folgenden Kapiteln vorgestellt.

\section{Hydrid-Bildung und resultierender spröder Bruch $\mid 2.3 .1$}

Die Grundlage für die Ausführungen in diesem Abschnitt sind in [Lyn12, $\left.\mathrm{RFM}^{+} 09\right] \mathrm{zu}$ finden. Für eine Gruppe von Metallen, zum Beispiel Vanadium, Zirkon, Niob, Tantal und Titan, ist der Mechanismus der Versprödung gut verstanden. Diese Materialien haben eine hohe Wasserstofflöslichkeit und bei höheren Wasserstoffkonzentrationen wird eine Hydrid-Phase gebildet. Der Bruchprozess ist spröde, da die Hydridphase spröde ist. Ist nun ein Riss im Material vorhanden, diffundiert der Wasserstoff in das Spannungsfeld der Rissspitze und hier findet lokal eine Phasenumwandlung in die Hydrid-Phase statt. Die Hydrid-Phase bricht dann spröde. Der Rissfortschritt stoppt sobald die Hydrid-Phase endet. Der Bruchprozess wird fortgesetzt sobald sich wieder eine Hydrid-Phase gebildet hat. Der Mechanismus der Wasserstoffversprödung durch Hydrid-Bildung wurde von Westlake 1969 [Wes69] vorgestellt und ist vielfach experimentell bestätigt worden. Beispielsweise 

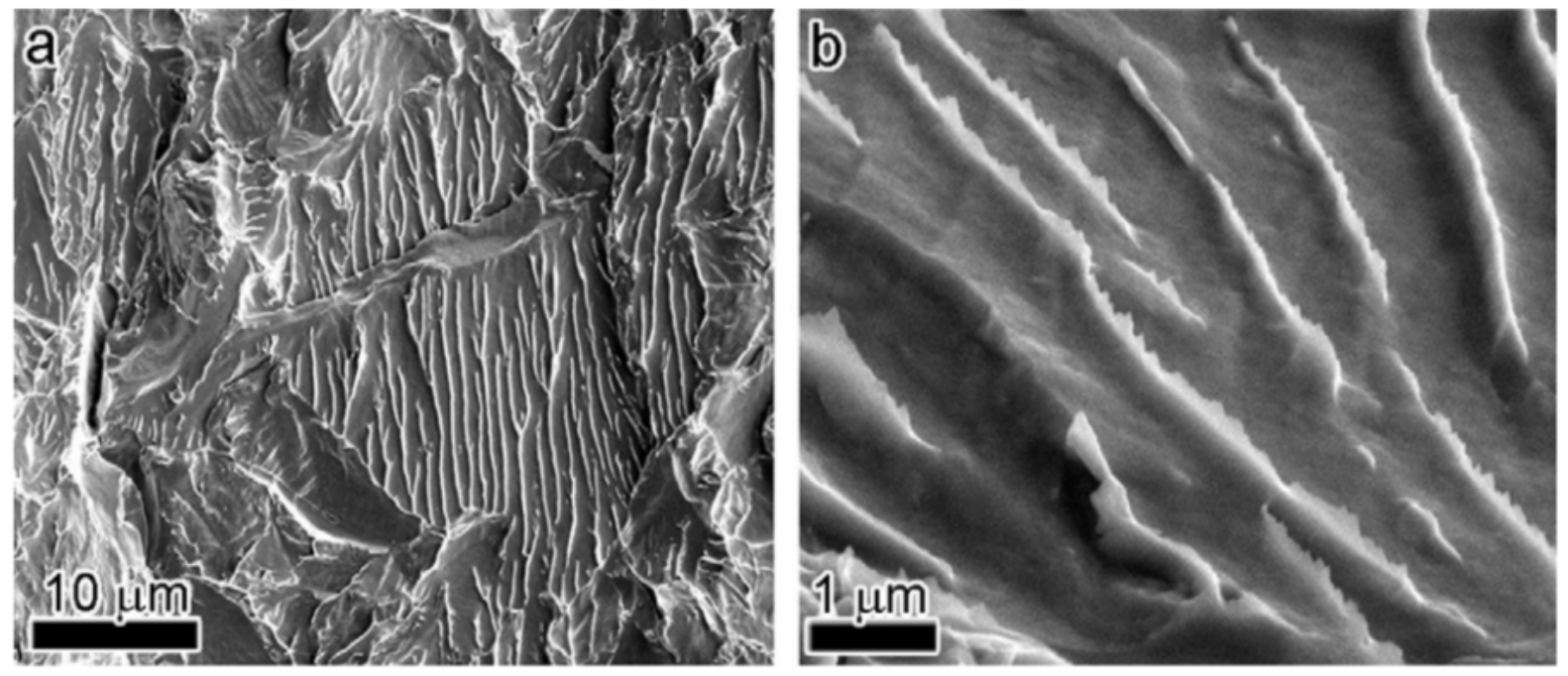

Abbildung 2.15: Reprint aus $\left[\mathrm{MFL}^{+} 11\right]$, Copyright 2011, mit Genehmigung von Elsevier. REM-Aufnahme der Bruchfläche eines Quasi-Spaltbruchs. In (a) sind die typischen Linien entlang der Rissausbreitung zu sehen. (b) ist eine Nahaufnahme dieser Linien (River Pattern). Man erkennt, dass die Linien sehr dünne Erhebungen sind, die im Elektronenmikroskop durchstrahlbar sind.

konnten Shih et al. [SRB88] in-situ im Transmissionselektronenmikroskopie (TEM) nachweisen, dass es in Titan unter bestimmten Bedingungen zu lokaler Hydrid-Bildung und infolgedessen zu einem spröden Bruch kommt. Für Eisen ist dieser Mechanismus nicht beobachtet worden, da es nur unter extremen Bedingungen (Wasserstoffdruck über 3,5 GPa) zu einer Hydrid-Bildung kommen kann [BHM91], daher wird der Mechanismus der Hydrid Bildung in dieser Arbeit nicht näher betrachtet.

\section{Herabsetzung der Kohäsion durch Wasserstoff (HEDE)}

Dieser Abschnitt orientiert sich an [Lyn12, KSS15]. HEDE beschreibt den Mechanismus, dass es durch Wasserstoff zu einer Herabsetzung der Bindungsstärke zwischen Eisenatomen kommt. Dadurch kann es unter Zugbelastung zu einer Separation von Ebenen kommen, bevor die kritische Schubspannung erreicht ist und Gleitprozesse aktiviert werden. Es findet somit ein Spaltbruch statt. Eine entsprechende Dekohäsionstheorie wurde erstmals 1926 vorgeschlagen [Pfe26] und im Anschluss unter anderem von Oriani [Ori72] weiterentwickelt. Hirth und Rice [HR80] geben eine thermodynamische Beschreibung dieses Bruchprozesses. 

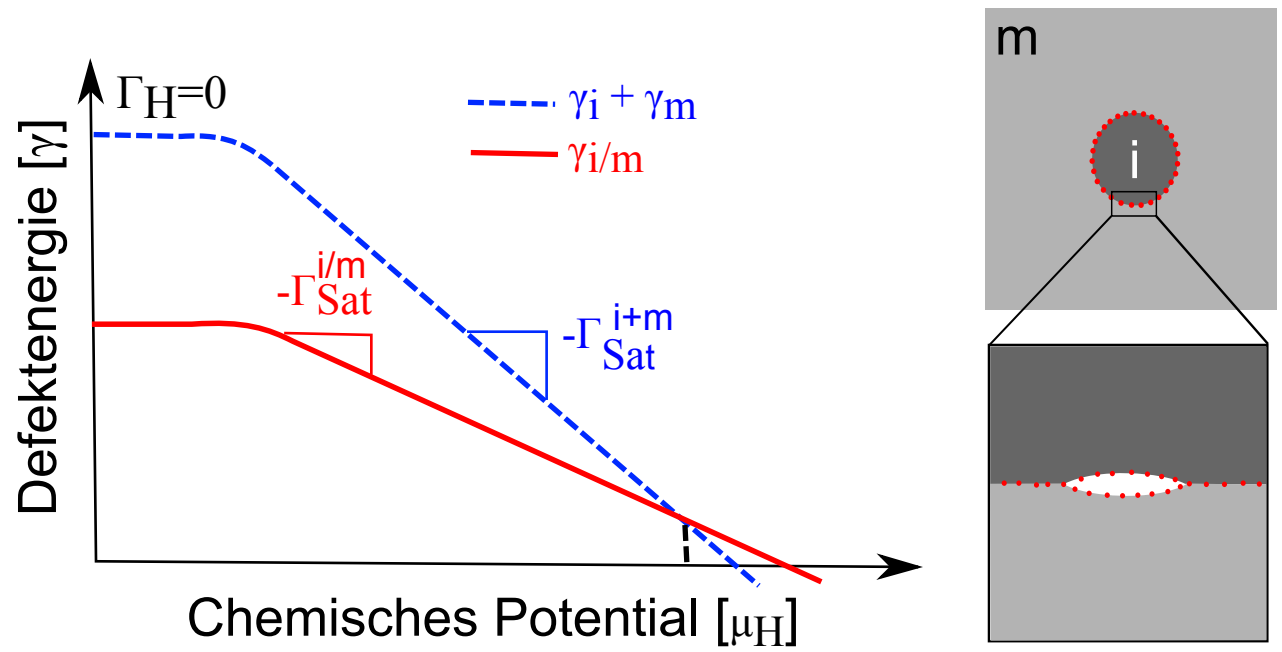

Abbildung 2.16: Nach Abbildung 2 in [KSS15]. Schematischer Verlauf der Defektenergie als Funktion des chemischen Potentials für die Grenzfläche zwischen Matrix und Einschluss (rot durchgezogen) und für die Summe der Oberflächenenergien von Matrix und Einschluss (blau gestrichelt). Nach dem Schnittpunkt der beiden ist die Spaltung der Grenzfläche energetisch günstiger.

Bestimmte Stellen im Material sind besonders anfällig für eine Wasserstoff-induzierte Dekohäsion. Es handelt sich um:

- Rissspitzen, denn hier liegt durch eine Adsorption von Wasserstoff auf der Rissfläche eine besonders hohe Wasserstoffkonzentration vor.

- Vor einer Rissspitze, wegen des Spannungsfeldes, welches durch den Riss verursacht wird.

- An der Grenzfläche von Einschlüssen zur umgebenden Eisenmatrix, da diese Grenzflächen Traps für Wasserstoff darstellen.

- Korngrenzen

Ein direkter Nachweis der Schwächung der atomaren Bildungen im Bulkmaterial ist nicht möglich, aber in einem Feldionenmikroskop konnte an der Oberfläche von Eisen eine Reduzierung der Verdampfungsfeldstärke in Wasserstoffatmosphäre nachgewiesen werden [WAM87]. In Abschnitt 2.1.4 wurde beschrieben, dass eine erhöhte Wasserstoffkonzentration an der Grenzfläche zu nichtmetallischen Einschlüssen im Vergleich zum Bulk vorliegt. Somit ist eine Dekohäsion an dieser Stelle wahrscheinlich. Thermodynamisch lässt sich dies im Rahmen des Defactants-Konzepts erklären, siehe dazu [KSS15]. Die Änderung der Grenzflächenenergie $\gamma_{i / m}$ von Eisen-Matrix und Einschluss lässt sich über Gleichung (2.4) beschreiben. Sie ist in Abbildung 2.16 schematisch skizziert. $\Gamma_{H}$ bezeichnet die Überschusskonzentration von Wasserstoff an der jeweils betrachteten Grenz- oder 
Oberfläche. Bei sehr kleinen chemischen Potentialen ist $\Gamma_{H}=0$ und somit ist die Grenzflächenenergie konstant. Bei einer Sättigung von Wasserstoff an der Grenzfläche ergibt sich ein linearer Abfall. Einen ähnlichen Verlauf zeigt die Summe der Oberflächenenergien von Matrix und Einschluss $\gamma_{i}+\gamma_{m}$, wobei die beiden Grenzflächen in Summe aufgrund der doppelten Fläche einen höheren Ausgangswert sowie eine höhere Sättigung aufweisen. Daher schneiden sich die beiden Kurven bei einem bestimmten chemischen Potential. Ab hier wird das Spalten von Matrix und Einschluss energetisch günstiger als das Fortbestehen der Grenzfläche. Es findet folglich eine Dekohäsion von Matrix und Einschluss statt.

\section{Erhöhung der lokalen Plastizität durch Wasserstoff (HELP) $\mid 2.3 .3$}

Ein zunächst widersprüchlich erscheinendes Modell ist das HELP Modell, welches beschreibt, dass Versetzungsbewegung durch Wasserstoff erleichtert wird. Dieser Abschnitt orientiert sich an [RFM+09, Lyn12]. Das Modell wurde 1972 von Beachem [Bea72] vorgestellt und ist in [BS94] zusammengefasst und diskutiert. Es wurden auf Bruchflächen von duktil gebrochenen Proben mit gelöstem Wasserstoff deutlich kleinere und flachere Mulden beobachtet als ohne gelösten Wasserstoff. Das führte zu der Interpretation, dass die duktile Verformung mit Wasserstoff deutlich stärker lokalisiert sei. Weitere Experimente, die eine erhöhte Versetzungsbewegung zeigen, wurden von Robertson durchgeführt [Rob01]. Hier wurde per in-situ Versuchen in einem TEM gezeigt, dass in einer Wasserstoff-Gasatmosphäre Versetzungsbewegung einsetzt und die Versetzungen näher zusammenrücken. Dadurch konnten Risse propagieren, die zuvor im Vakuum stabil waren, wobei die Dehnung konstant gehalten wurde.

Dieses Modell kann ebenfalls eine makroskopische Versprödung des Materials erklären, obwohl es mikroskopisch eine Erhöhung der Duktilität beschreibt. Der Grund ist, dass es zu einer starken Lokalisation der plastischen Verformung kommt. Das geht so weit, dass ein Bruch eine glatt erscheinende Bruchfläche aufweist und erst bei hoher Vergrößerung im REM können Mulden in der Größenordnung von 10 bis $100 \mathrm{~nm}$ nachgewiesen werden [MRS11]. Da die Bruchfläche makroskopisch, das heißt bei niedrigerer Vergrößerung glatt aussieht, ist das Erscheinungsbild einem Spaltbruch sehr ähnlich. 


\section{Experimentelle Methoden}

In diesem Kapitel werden die experimentellen Mess- und Analysemethoden vorgestellt, die zur Charakterisierung der Proben nach der Wasserstoffbeladung zum Einsatz kommen. Dabei wird auf die Beschreibung der grundsätzlichen Funktionsweise der Geräte, die in der Materialphysik üblich sind, wie zum Beispiel der Elektronenmikroskope, verzichtet. Für diese Geräte wird ausgeführt, wie sie speziell in dieser Arbeit eingesetzt wurden.

\section{Elektrochemische Wasserstoffbeladung $\mid 3.1$}

Die Eisenproben wurden elektrochemisch bei konstantem Strom beladen. Dafür wird die Probe mit einem Platinhaken in den Elektrolyten getaucht und als Kathode geschaltet, siehe Abbildung 3.1. Die Gegenelektrode besteht aus Platin. Es wird für den Haken und die Gegenelektrode Platin verwendet, da dies in den verwendeten elektrochemischen Prozessen inert ist und eine hohe elektrische Leitfähigkeit besitzt.

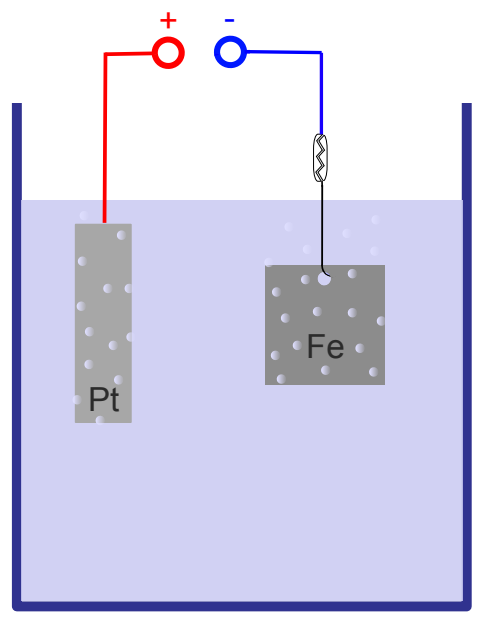

Abbildung 3.1: Schematische Darstellung des Aufbaus zur elektrochemischen Wasserstoffbeladung. Probe taucht in den Elektrolyten, dabei ist sie als Kathode an eine konstante Stromquelle angeschlossen. Die Gegenelektrode ist aus Platin.

$$
\begin{array}{l|l}
250 \mathrm{~g} & \mathrm{H}_{2} \mathrm{O} \\
2,45 \mathrm{~g} & \mathrm{H}_{2} \mathrm{SO}_{4} \\
0,25 \mathrm{~g} & \mathrm{NH}_{4} \mathrm{SCN}
\end{array}
$$

Tabelle 3.1: Zusammensetzung des Elektrolyten, der zur elektrochemischen Wasserstoffbeladung eingesetzt wurde $\left[\mathrm{SKH}^{+} 11\right]$.

Der verwendete Elektrolyt besteht aus verdünnter Schwefelsäure mit 0,1 gew. \% Ammoniumthiocyanat $\left(\mathrm{NH}_{4} \mathrm{SCN}\right)$ als Wasserstoffpromoter, vergleiche Tabelle 3.1, dieses Rezept 
wurde aus Suzuki et al. [SKH $\left.{ }^{+} 11\right]$ entnommen. Es wurden Stromdichten im Bereich von $0,01-150 \mathrm{~mA} / \mathrm{cm}^{2}$ verwendet, wobei die Zeit der Beladung einige Minuten bis zu 5 Stunden betrug. Bei einer typischen Beladung wurde 1 Stunde bei $50 \mathrm{~mA} / \mathrm{cm}^{2}$ beladen.

\section{Das Rasterelektronenmikroskop (REM)}

Zur Untersuchung der Proben nach der Wasserstoffbeladung wird ein FEI Nova NanoSEM Rasterelektronenmikroskop eingesetzt. Grundlagen eines REM werden in [Haf07] näher erläutert. Es bietet im Vergleich zu einem optischen Mikroskop den Vorteil der Tiefenschärfe, sodass Bruchflächen mit Höhenunterschieden von einigen Millimetern dargestellt werden können. Des Weiteren sind an diesem Gerät zusätzliche Detektoren installiert. In dieser Arbeit kommt energiedispersive Röntgenspektroskopie (Energy Dispersive X-ray Spectroscopy, EDX) zur chemischen Charakterisierung und Elektronenrückstreubeugung (Electron Backscatter Diffraction, EBSD) zur Darstellung der lokalen kristallographischen Orientierung der Probenoberfläche zum Einsatz. Diese beiden Techniken werden in den folgenden zwei Abschnitten näher erläutert.

\section{Energiedispersive Röntgenspektroskopie (EDX)}

In dieser Arbeit wurde EDX verwendet, um die chemische Zusammensetzung von Einschlüssen im Eisen zu bestimmen. Diese Einschlüsse spielen eine entscheidende Rolle bei der Rissinitiierung. Es wird ein Oxford Xmax 80 EDS System verwendet. Die Steuerung und Auswertung erfolgt mit INCA software for microanalysis.

\section{Elektronenrückstreubeugung (EBSD)}

Um ortsaufgelöste Informationen über kristallographische Orientierungen zu erhalten, wird EBSD als zusätzliche Technik innerhalb des REM verwendet. Details zur Funktionsweise dieser Technik können [Len09] entnommen werden. Es wird durch zweifache Streuung in der Probe ein Beugungsbild bestehend aus Kikuchi-Linien aufgenommen und mithilfe einer Software kann daraus ortsaufgelöst die dreidimensionale Orientierung bestimmt werden. Diese Orientierung an einem Punkt kann über drei sogenannte Euler-Winkel angegeben werden. Die Definition der Euler-Winkel können Abbildung 3.2 entnommen werden. Für die Darstellung der EBSD-Messungen werden in dieser Arbeit sogenannte Euler-Karten verwendet. Das bedeutet die dargestellte Farbe setzt sich jeweils aus rot, grün und blau zusammen und jede dieser Farben repräsentiert einen Euler-Winkel. Diese Darstellung hat den Vorteil, dass Rotationen des Materials in alle Raumrichtungen dargestellt werden. Ein Beispiel für eine Euler-Karte ist in Abbildung $3.3 \mathrm{zu}$ sehen. 

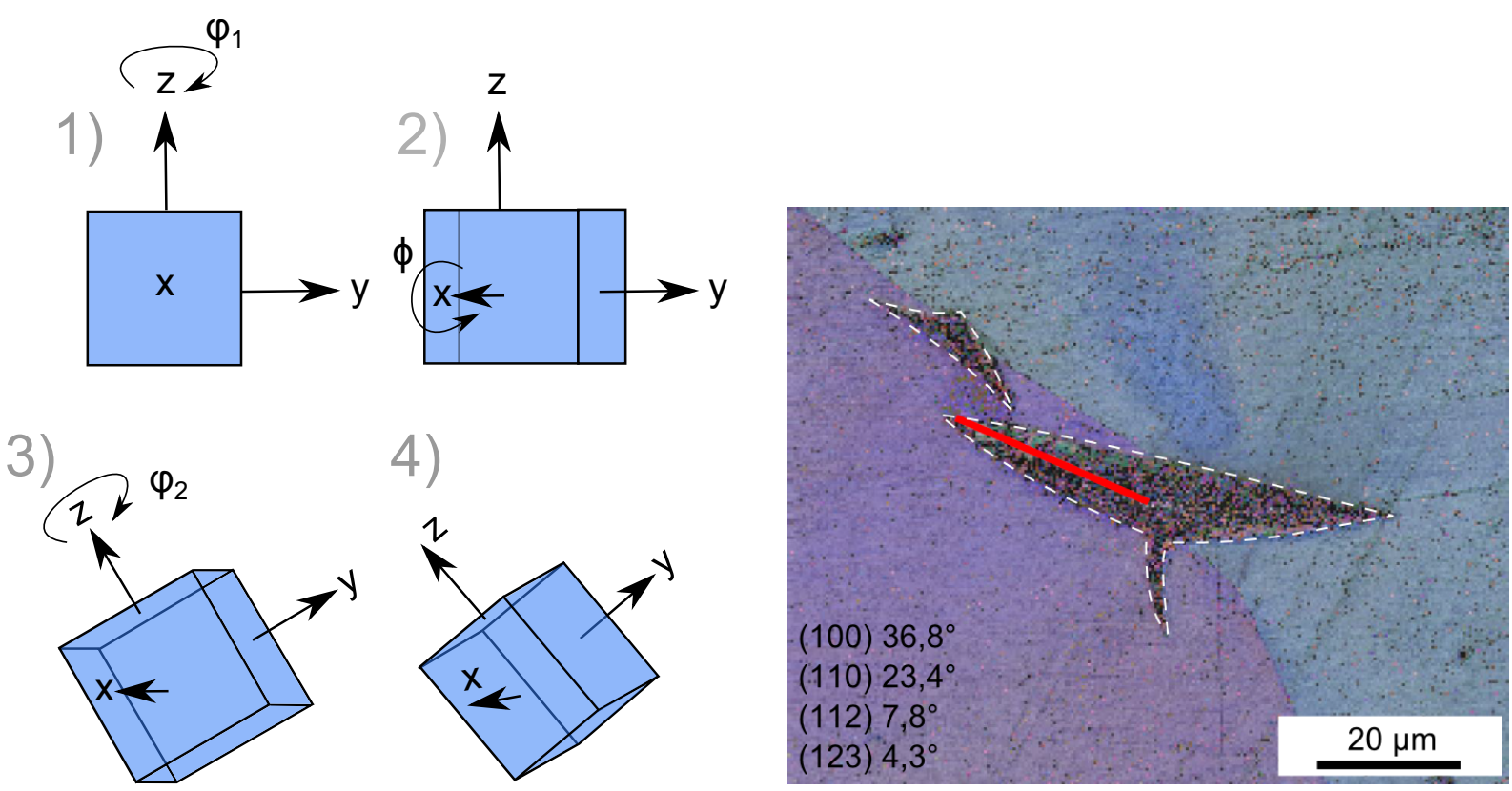

Abbildung 3.2: Zeichnung angefertigt nach [MS07], S.41. Schematische Abbildung zur Veranschaulichung der Euler-Winkel nach der Bunge-Konvention. Es wird um die z-Achse gedreht, danach um die $\mathrm{x}$-Achse und abschließend noch einmal um die z-Achse. Dadurch lässt sich jede Orientierung in eine beliebige andere überführen.

Abbildung 3.3: Messung und Auswertung erfolgte in Kooperation mit May L. Martin. In der EBSD-Euler-Karte ist der Umriss des Risses mit einer weißen gestrichelten Linie nachgezeichnet. Die Ebene, die als Rissebene angenommen wird, ist mit einer durchgezogenen roten Linie gekennzeichnet. Sie wurde per Hand mittig in den Riss gezeichnet. Die Winkel zu potentiellen Rissebenen sind angegeben.

Der Fokus in dieser Arbeit wurde auf die Bestimmung der kristallographischen Ebenen, entlang der die Risse verlaufen, gelegt. Dabei ist zu beachten, dass man den Querschliff der Probe betrachtet und dadurch den dreidimensionalen Verlauf des Risses nicht kennt. Daher vergleicht man spezielle kristallographische Ebenen aus dem umliegenden Material mit der in den Riss eingezeichneten Linie. Die Orientierung der Ebenen im umliegenden Material kann aus den Euler-Winkeln bestimmt werden, die aus der Messung an jeden Punkt ausgelesen werden können. Potentielle Rissebenen sind zum Beispiel Spaltebenen und Gleitebenen von Eisen. Da die Risse oft stark geöffnet sind, wird jeweils die Linie mittig im Riss als Rissebene angenommen. Ein Beispiel, wie die Rissebene bestimmt wird, ist in Abbildung 3.3 zu sehen. Es wird per Hand eine Ebene mittig in den Riss gezeichnet und anschließend werden die Winkel zu verschiedenen kristallographischen Ebenen berechnet. Die Orientierung dieser Ebenen wird aus den Euler-Winkeln des umgebenden Materials ausgelesen. Da die Ermittlung der Rissebene aufgrund der starken Öffnung der Risse nur auf einige Grad genau ist, werden Ebenen, die $\pm 10^{\circ}$ zur Rissebene liegen, als potentielle Rissebenen in Betracht gezogen. Es können so vor allem Rissebenen ausgeschlossen 
werden, aber bei einer Auswertung von mehreren Rissen kann auch auf bevorzugte Rissebenen geschlossen werden. Zusätzlich kann mit EBSD die Kornrotation des umliegenden Materials um die Risse bestimmt werden, was ein Maß für die plastische Verformung um einen Riss darstellt.

\section{Focused Ion Beam (FIB) zur Herstellung von TEM-Lamellen}

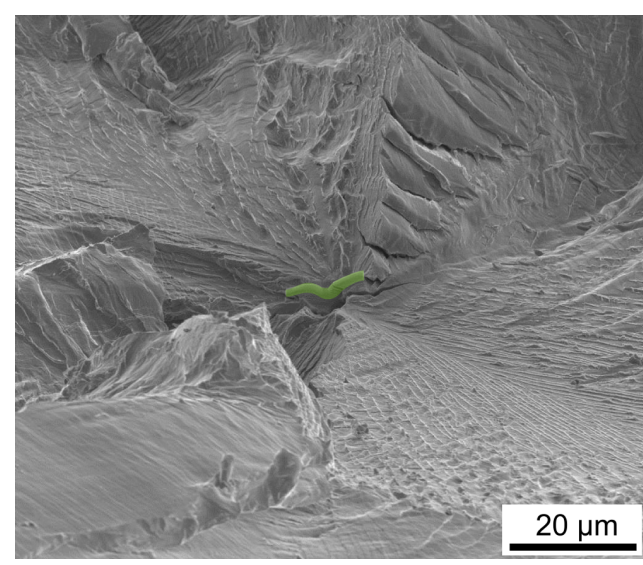

Abbildung 3.4: REM-Bild: Beispiel einer Platin-Deposition (grün eingefärbt), die zum Schutz beim späteren Ausdünnen der Lamelle dient, über einer Initiierungsstelle eines Risses.
Zur Untersuchung der Defektstruktur des Materials wurden TEM-Lamellen aus dem Material, das Risse und Blister umgibt, extrahiert. Dafür wurde ein FEI Nova Nano Lab 600 Focused Ion Beam (FIB) benutzt. Die Lamellen-Extraktion erfolgt nach der Lift-out-Technik. Eine Beschreibung dieser Technik ist in $\left[\mathrm{GKA}^{+} 02\right] \mathrm{zu}$ finden und wurde von Overwijk et al. [OVB93] entwickelt. Es entsteht eine für hochenergetische Elektronen transparente Lamelle von etwa $7 \mu \mathrm{m}$ Breite und $5 \mu \mathrm{m}$ Tiefe. Dafür wird zunächst ein etwa $2 \mu \mathrm{m}$ dicker Platinstreifen auf der Oberfläche deponiert und unterhalb davon wird senkrecht zur Oberfläche die Lamelle extrahiert. Das Platin dient zum Schutz der Lamelle beim sich anschließenden Prozess zum Ausdünnen der Lamelle mit dem Gallium-Ionen-Strahl.

Die Schwierigkeit bei der Lamellenpräparation aus Bruchflächen ${ }^{1}$ sind die extremen Höhenunterschiede der Oberfläche (bis zu einigen Millimetern). Vor allem die Rissinitiierungsstellen liegen in Tälern, da diese im Zentrum von weit geöffneten Rissen liegen, wie in Abschnitt 5.1.2 näher beschrieben ist. Ein Beispiel während des Entstehungsprozesses einer TEM-Lamelle, die aus einem Tal extrahiert wurde, ist in Abbildung 3.4 zu sehen.

\section{Transmissionselektronenmikroskop (TEM) mit verwendeter Rotationskalibrierung}

Die Untersuchung der TEM-Lamellen erfolgte in einem TEM CM12 der Firma Philips. Es wurden jeweils Hellfeldaufnahmen und teilweise Beugungsaufnahmen angefertigt. Um spezielle Merkmale im Hellfeldbild auf kristallographische Richtungen zurückführen zu können, wurde auf eine Rotationskalibrierung zwischen Beugungs- und Realbild zurückgegrif-

\footnotetext{
${ }^{1}$ Eine detaillierte Einführung in die Lamellenpräparation aus Bruchflächen gab mir May L. Martin, vielen Dank dafür!
} 


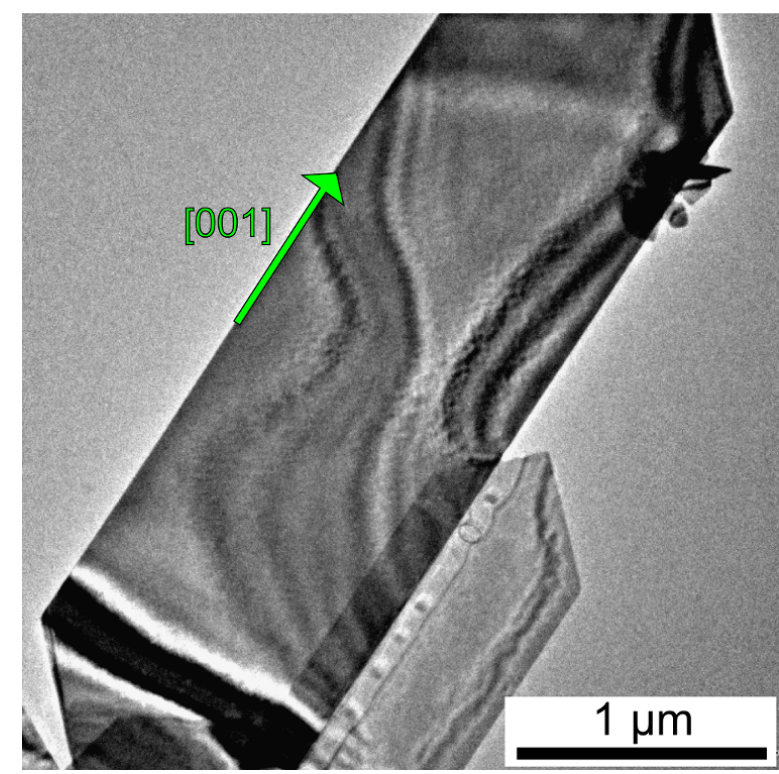

Abbildung 3.5: Bild wurde in Zusammenarbeit mit May L. Martin erstellt. HellfeldBild zur Rotationskalibrierung. Zu sehen ist ein Molybdäntrioxid-Kristall.

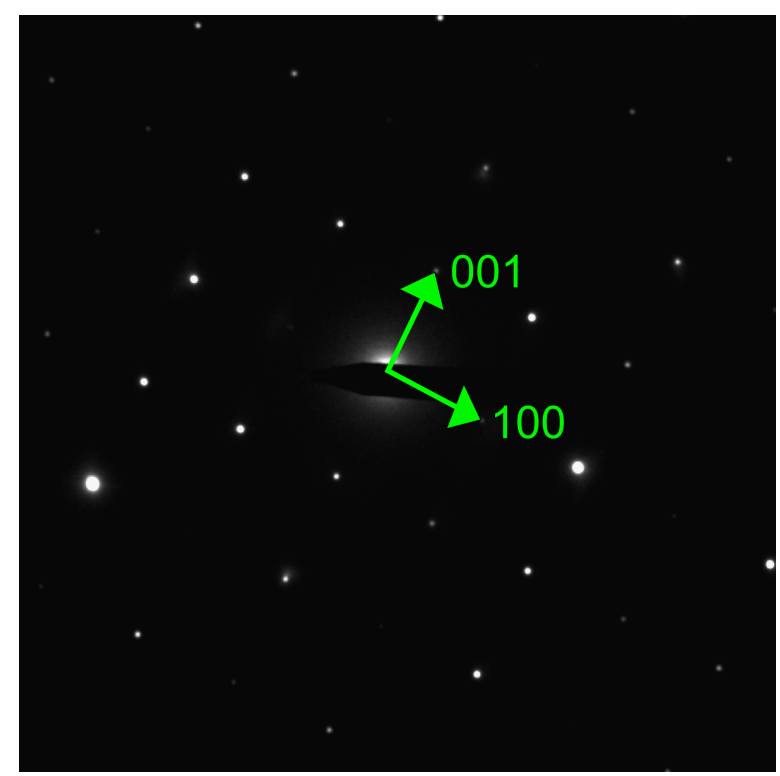

Abbildung 3.6: Bild wurde in Zusammenarbeit mit May L. Martin erstellt. Beugungsbild des Molybdäntrioxid-Kristall aus Abbildung 3.5. Zonenachse ist [010].

fen. Diese entstand in Zusammenarbeit mit May L. Martin. Für diese Rotationskalibrierung kam eine Eichprobe der Firma Plano, die aus Molybdäntrioxid-Kristallen auf einem TEM-Netzchen besteht, zum Einsatz. Molybdäntrioxid hat eine pseudo-orthorombische Struktur mit den Gitterparametern $a=397 \mathrm{pm}, b=1383 \mathrm{pm}$ und $c=370 \mathrm{pm}$ [NC92]. Die Kristalle wachsen "Band-förmig", das heißt in [010] Richtung sind sie elektronentransparent und in [001] Richtung gestreckt im Vergleich zur [100] Richtung [NC92]. Ein Hellfeldbild ist in Abbildung 3.5 abgebildet und ein Beugungsbild dieses Kristalls mit einer Zonenachse von [010] ist in Abbildung $3.6 \mathrm{zu}$ sehen. Ein Abgleich der Richtungen führt zu einer Rotationskalibrierung, die abhängig von der Vergrößerung und der verwendeten Kameralänge ist. Diese Kalibrierung wurde verwendet, wenn in Hellfeldbilder kristallographische Richtungen angegeben sind.

\section{Wasserstoffanalyse $\mid 3.5$}

Im Rahmen dieser Arbeit wurde ein Wasserstoff-Analysator Galileo G8 der Firma Bruker eingesetzt. Dieser dient zur Bestimmung des Wasserstoffgehaltes in Feststoffen. Die Messung basiert auf der Trägergas-Schmelzextraktionsmethode.

Die Wasserstoffanalyse wurde eingesetzt, um zu ermitteln, wie viel Wasserstoff in Abhängigkeit von der Beladestromdichte in der Probe gelöst wird. Dabei kann Wasserstoff, der als Gas in den Rissen vorliegt, nicht von Wasserstoff, der im Eisengitter gelöst ist, 
unterschieden werden. Der verwendete Analysator muss mit Proben bekannter Wasserstoffkonzentration geeicht werden. Dafür standen Eichproben AR 555 der Firma HRT zur Verfügung, die einen Wasserstoffgehalt von 3,4 ppm haben. Des Weiteren spricht bei höheren Wasserstoffmengen ein zweiter Detektor an, dieser wird durch wasserstoffbeladene Silberbleche geeicht. Ein kleines Blech wird im Analysator gemessen, wobei die Größe so gewählt wird, dass nur der Detektor für geringe Mengen anspricht. Daher ist die Konzentration im Blech bekannt, sie liegt bei etwa 100 ppm. Zur Eichung des Detektors für größere Mengen an Wasserstoff, wird ein deutlich größeres Stück des Bleches gemessen. Aufgrund dieser Eichung kann die nominelle Genauigkeit des Gerätes nicht erreicht werden. Durch eine Messreihe wurde der Fehler aufgrund von Abweichungen zwischen verschiedenen Messungen auf 10\% des gemessenen Wertes abgeschätzt.

\section{Dichtemessung zur Bestimmung des Gesamtvolumens der Risse}

Die Dichtemessung erfolgt nach dem archimedischen Prinzip, das heißt es wird die Kraft, die auf die Probe wirkt in Luft und in einer Flüssigkeit gemessen. Die Kraft setzt sich aus der Gewichts- und der Auftriebskraft zusammen. Aufgrund des Auftriebs, den die Probe in der Flüssigkeit erfährt, kann bei bekannter Flüssigkeitsdichte auf die Dichte der Probe geschlossen werden, wobei auch der Auftrieb der Aufhängung der Probe beachtet werden muss:

$$
\varrho_{P}=\frac{F_{L}}{F_{L}-\left(F_{F l}-F_{H}\right)} \cdot \varrho_{F l} .
$$

$\varrho_{P}$ ist die Dichte der Probe und $\varrho_{F l}$ ist die Dichte der Flüssigkeit. $F_{L}$ ist die Kraft, die die Probe in Luft und $F_{F l}$ in der Flüssigkeit erfährt. $F_{H}$ beschreibt die Auftriebskraft des Hakens, das heißt die Differenz zwischen den gemessenen Kräften des Hakens in Luft und in der Flüssigkeit. Alle Kräfte werden von der Waage als Masse $m=F / g$ ausgegeben, $g$ bezeichnet dabei die Erdbeschleunigung. Der experimentelle Aufbau der Dichtemessung wurde in der Bachelorarbeit von Annegret Lehmberg entwickelt [Leh14]. Als Schwereflüssigkeit zur Auftriebsmessung wird Natriumpolywolframat in wässriger Lösung verwendet, das mit Chromsalzen stabilisiert wurde. Diese Flüssigkeit hat je nach gelöster Konzentration an Natriumpolywolframat eine Dichte bis zu $3,1 \mathrm{~g} / \mathrm{cm}^{3}$ und die Chromsalze verhindern, dass die Probe von der Lösung angegriffen wird. ${ }^{2}$ Im Rahmen dieser Arbeit wird eine Lösung mit einer Dichte von $3,0 \mathrm{~g} / \mathrm{cm}^{3}$ verwendet. Die Fehler dieser Methode werden im Folgenden abgeschätzt. Die Gewichtskraft der Probe in Luft $F_{L} / g$ ist durch die Genauigkeit der verwendeten Halb-Mikrowaage ${ }^{3}$, die eine Auflösung von $10 \mu \mathrm{g}$ hat, bestimmt. Der Fehler wird mit $\pm 50 \mu \mathrm{g}$ abgeschätzt. Messungen an verschiedenen Tagen, auch nachdem die Probe in der Schwereflüssigkeit war, blieben immer innerhalb dieses Fehlers. Die Kraft

\footnotetext{
${ }^{2}$ TC-Tungsten Compounds GmbH http://www.heavy-liquid.com

${ }^{3}$ Sartorius Halb-Mikrowaage Research R 180 D-*D1
} 
auf die Probe in der Schwereflüssigkeit ist durch den Auftrieb der Probe bestimmt. Das Kräftegleichgewicht in der Flüssigkeit stellt sich erst langsam ein, sodass die Kraft auf die Probe in der Flüssigkeit erst nach etwa einer Stunde stabil ist. Der Fehler wird durch die Fluktuationen um den sich einstellenden Mittelwert bestimmt.

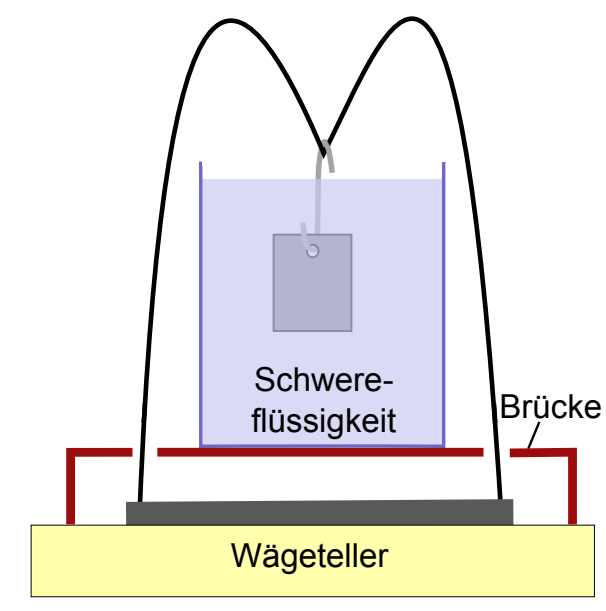

Abbildung 3.7: Schematische Darstellung der Dichtemessung: Die Probe taucht während der Messung in die Flüssigkeit, die auf einer Brücke steht. Um die Dichte zu bestimmen wird zusätzlich die Kraft auf die Probe in Luft und der Auftrieb des Haken gemessen.

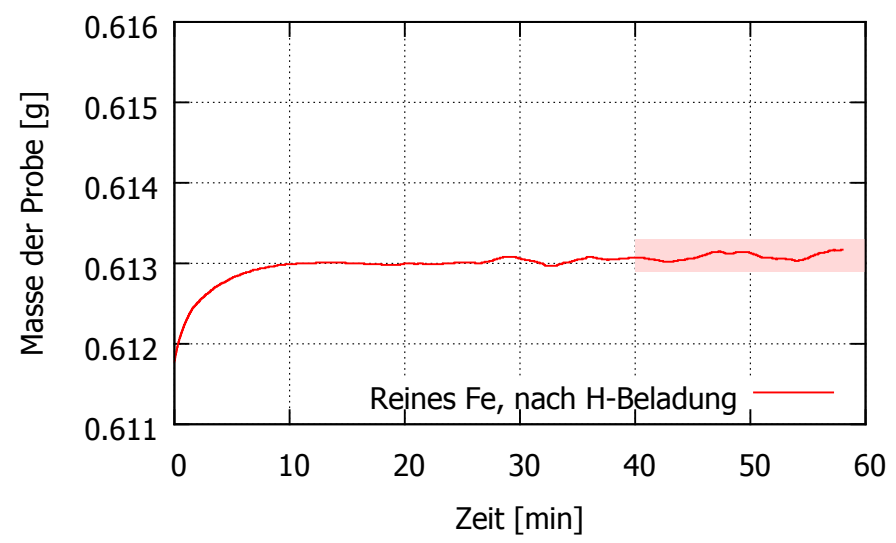

Abbildung 3.8: Auftriebsmessung einer Eisenprobe in der Schwereflüssigkeit in Abhängigkeit der Zeit. Nach etwa einer halben Stunde stellt sich ein Gleichgewichtswert ein, dessen Fehler mit der hell rot hinterlegten Box abgeschätzt wird.

Die Messung einer Eisenprobe ist in Abbildung 3.8 gezeigt. Der hell-rot hinterlegte Bereich zeigt den Fehler, der in dieser Messung mit $\pm 2 \mathrm{mg}$ abgeschätzt wurde. Das Auftriebsgewicht des Hakens wird durch Abstreifen der Probe und anschließende Messung bestimmt. Der Fehler bei dieser Messung ist, wie bei der Messung der Probe in der Flüssigkeit, $\pm 2 \mathrm{mg}$. Des Weiteren ist die Dichte der Flüssigkeit eventuell fehlerbehaftet, daher wird die Dichte der Eisenproben bestimmt und mit dem Wert nach der Beladung verglichen, anstatt den Literaturwert der Dichte von Eisen zu verwenden. Verschiedene Messungen an reinem Eisen ergaben:

$$
\varrho_{F e}=(7,846 \pm 0,008) \frac{\mathrm{g}}{\mathrm{cm}^{3}} \quad \varrho_{F e, \text { Literatur }}=7,874 \frac{\mathrm{g}}{\mathrm{cm}^{3}}
$$

Die Abweichung zum Literaturwert ${ }^{4}$ liegt nicht innerhalb des Fehlerbereiches, was höchstwahrscheinlich an einer fehlerhaften Angabe der Dichte der Flüssigkeit liegt, denn der gemessene Wert ist bei verschiedenen Messungen gut reproduzierbar. In dieser Arbeit soll

\footnotetext{
${ }^{4}$ http://www.periodensystem. info/elemente/eisen/, aufgerufen 26.07.2016
} 
die Dichtemessung zur Bestimmung des Rissvolumens $V_{\text {Risse }}$ in Eisenproben eingesetzt werden:

$$
V_{\text {Risse }}=m_{L}\left(\frac{1}{\varrho_{P}}-\frac{1}{\varrho_{F e}}\right),
$$

wobei $\varrho_{P}$ in diesem Fall die Dichte der Probe nach der Wasserstoffbeladung und $m_{L}$ die Masse der Probe in Luft bezeichnet. In dieser Gleichung wird für $\varrho_{F e}$ die Messung einer Eisenprobe verwendet und nicht der Literaturwert, somit kann das Volumen der Risse auch ohne Kenntnis der Dichte der Flüssigkeit berechnet werden.

\section{Messung des Wasserstoffaustritts aus der Probe nach Beladung}

Zur Ermittlung des Wasserstoffdrucks in den Rissen wird nach der Beladung eine Druckmessung in einer Vakuum-Kammer durchgeführt, in die der Wasserstoff nach der Beladung ausgast. Dafür wird die Probe nach der elektrochemischen Wasserstoffbeladung in eine Vakuumanlage eingebaut. Dieser Vorgang dauert etwa 2 Minuten, wodurch ein wenig austretender Wasserstoff für die Messung verloren geht. Die Probenkammer wird durch Öffnen eines Ventils zur Turbopumpe auf ein Vakuum von $10^{-5}$ mbar abgepumpt, wieder verschlossen und die Druckmessung wird gestartet, vergleiche Abbildung 3.9.

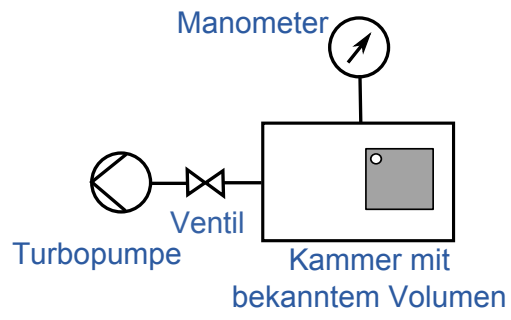

Abbildung 3.9: Schematische Darstellung der Messung des Wasserstoffaustritts. Die Probe wird nach der Beladung in ein geschlossenes Volumen eingebracht, ein Vakuum wird erzeugt und der Druckanstieg in der Kammer wird aufgezeichnet. Die Vakuumanlage wurde konstruiert und aufgebaut von Magnus Hamm, Universität Göttingen.

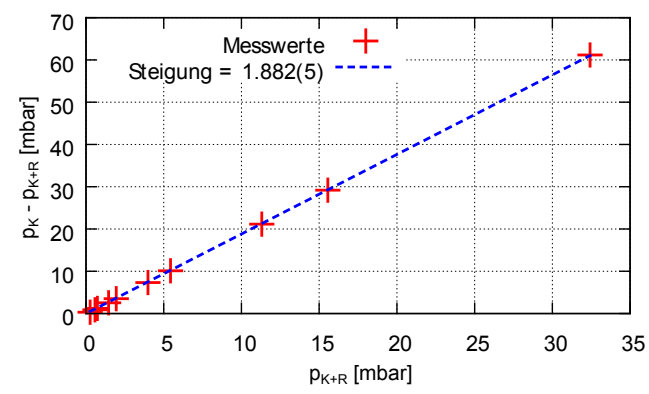

Abbildung 3.10: Auftragung zur Ermittlung des Kammervolumens, in die der Wasserstoff aus der Probe entgast. Ein Rohr bekannten Volumens wird angeschlossen und der Druck in der Kammer $p_{K}$ und in dem Volumen aus Kammer und Rohr $p_{K+R}$ wird gemessen. Lineare Regression liefert $V_{R} / V_{K}$.

Das Volumen der Kammer $V_{K}$ wird durch Anbau eines Rohrs mit einem bekannten Volumen von $V_{R}=162,3 \pm 1,5 \mathrm{~cm}^{3}$, das zwischen Turbopumpe und Kammer angebaut wird, ermittelt. Dafür wird jeweils ein Druck in der Kammer $p_{K}=p\left(V_{K}\right)$ eingestellt, ein Ventil zum Rohr geöffnet und der Druck gemessen, der sich in Kammer plus Rohr 
$p_{K+V}=p\left(V_{K}+V_{R}\right)$ einstellt. Dieser Vorgang wird für verschiedene Drücke wiederholt. Nun kann von dem Volumen des Rohrs auf das Volumen der Kammer inklusive Leitung zum Manometer etc. geschlossen werden. Dafür wird der Druck im Volumen aus der Kammer und dem Rohr $p_{K+R}$ gegen die Differenz der gemessenen Drücke $p_{K}-p_{K+R}$ aufgetragen und eine lineare Regression mit Steigung $m$ durchgeführt. Das Volumen der Kammer ergibt sich durch Gleichsetzten der Stoffmenge im idealen Gasgesetz:

$$
p_{K} \cdot V_{K}=p_{K+R} \cdot\left(V_{K}+V_{R}\right) \quad \Rightarrow \quad V_{K}=\frac{m}{V_{R}}
$$

Es ergibt sich ein Kammervolumen von: $V_{K}=(86,2 \pm 0,9) \mathrm{cm}^{3}$.

Der Austritt des Wasserstoffs aus der Probe erfolgt über mehrere Tage, sodass eine Stabilitätsmessung durchgeführt wurde, die zeigt, inwiefern die Vakuumanlage den Druck in der Kammer halten kann, ohne dass Wasserstoff zur Vakuumseite entweicht oder Luft von außen den Druck erhöht. Dazu wird in der Kammer ein bestimmter Wasserstoffdruck eingestellt und eine Druckmessung über mehrere Tage durchgeführt. Das Ergebnis ist in Abbildung 3.11 aufgetragen. Der Anstieg bei einem spezifischen Wasserstoffdruck wird jeweils linear im Bereich von 5 bis 25 Stunden gefittet. Die Ergebnisse sind in Tabelle 3.2 zusammengestellt. Man kann erkennen, dass jeweils ein Druckanstieg in der Kammer von einigen $\mu$ bar pro Stunde vorliegt. Dabei lässt sich zwischen den Messungen kein Trend erkennen. Daher kann der Unterschied zwischen den Messungen nur zum Beispiel auf

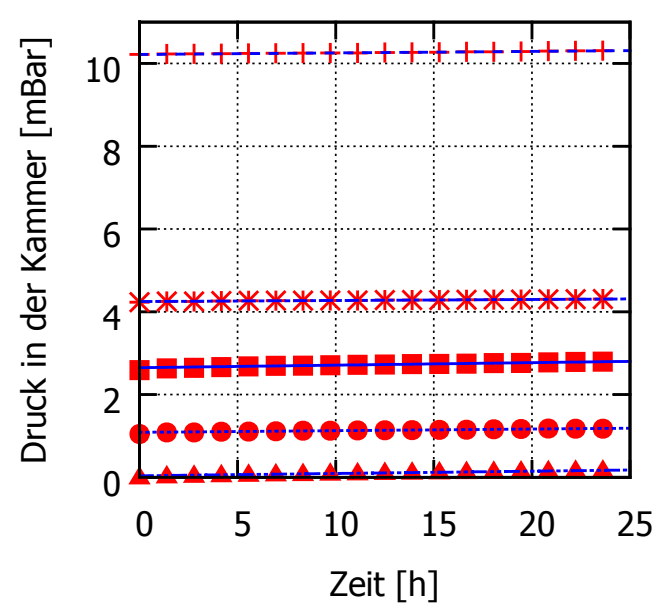

Abbildung 3.11: Messung zur Stabilität des Drucks in der Kammer. Es wurde ein Wasserstoffdruck bzw. Vakuum eingestellt und der Druck aufgezeichnet. Die linearen Fits erfolgten jeweils von 5 bis 25 Stunden.

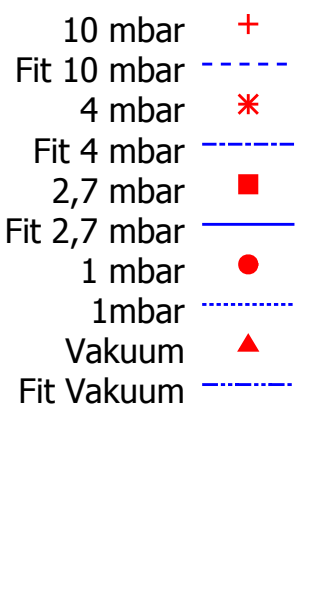

Ergebnisse der Stabilitätsmessung in Abbildung 3.11. Zur Korrektur der Druckmessungen wird ein Mittelwert über die ermittelten Steigungen gebildet. 
eine unterschiedlich gute Abdichtung der Kammer zurückgeführt werden. Um eine Korrekturgerade, die von den Druckmessungen abgezogen wird, zu ermitteln, wird über alle gemessenen Steigungen der Stabilitätsmessungen gemittelt. Es ergibt sich ein Wert von:

$$
m_{\mathrm{korr}}=(4,2 \pm 1,4) \frac{\mu \mathrm{bar}}{\mathrm{h}}
$$

\section{Permeationsmessungen mittels eines KircTec Sensors}

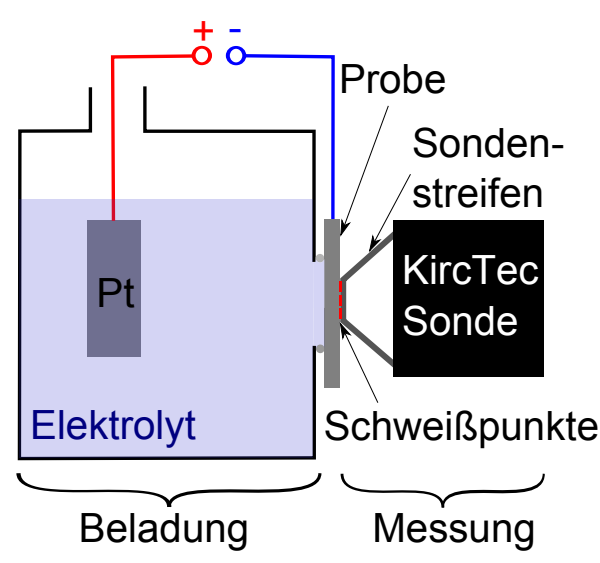

Abbildung 3.12: Schematische Darstellung des Aufbau zur Permeationsmessung. Die elektrochemische Beladung erfolgt von der linken Seite. Die Messung des penetrierten Wasserstoffs ist durch einen KircTec Sensor, der durch Punktschweißen mit der Probe verbunden ist, realisiert.
Die Kinetik der Wasserstoffbeladung wurde mit Permeationsexperimenten untersucht. Der dafür verwendete Aufbau ist in Abbildung 3.12 schematisch gezeigt. Die Probe wird dabei, wie in Abschnitt 3.1 dargestellt, elektrochemisch mit Wasserstoff beladen. Allerdings wird nicht die gesamte Probe in den Elektrolyten getaucht, sondern nur eine Fläche von $\sim 2,5 \mathrm{~cm}^{2}$ dem Elektrolyten ausgesetzt, sodass die Beladung auf einer definierten Fläche einer Probenseite erfolgt. Auf der anderen Probenseite (rechts in Abbildung 3.12) wird mit einem KircTec Sensor der Wasserstoff gemessen [Kir11], der durch die Probe diffundiert ist. Dafür wird ein Sondenstreifen, der aus einem Wasserstoffaffinerem Material als Eisen besteht, durch Punktschweißen mit der Eisen-Probe verbunden, sodass die Wasserstoffkonzentration in der Probe direkt hinter einem Schweißpunkt auf Null abfällt. So kann während der elektrochemischen Beladung die Wasserstoffpermeation durch die Probe gemessen werden.

Bei einer Permeationsmessung wird eine Probe stufen-

weise beladen. Das bedeutet, dass bei den ersten Beladungsschritten noch keine Risse und Blister vorhanden sind. Durch dieses Vorgehen kann ermittelt werden kann, wie sich die Riss- und Blisterbildung auf die Permeation des Wasserstoffs durch die Probe auswirkt.

\section{Weitere verwendete Messmethoden}

- Zur Beobachtung des Blisterwachstums während der Beladung wurde die USBMikroskopkamera DNT DigiMicro 2.0 Scale mit bis zu 200-facher Vergrößerung eingesetzt. Es wurden Bilderserien aufgenommen, indem alle $10 \mathrm{~s}$ ein Bild der Probe erstellt wurde. Die Probe befindet sich während der Aufnahme im Elektrolyten 
und Wasserstoff rekombiniert auf der Oberfläche zu Gas, dadurch wird die Qualität der Aufnahme herabgesetzt. In der Bilderserie in Abschnitt 5.1.5 sind Bilder im zeitlichen Abstand von circa einer Minute zu sehen. Es wurde jeweils ein Bild ausgewählt, in dem keine große Gasblase den Blister verdeckt, daher variiert der zeitliche Abstand leicht.

- Zur Messung der Höhenprofile von Blistern wurde das Profilometer Dektak 150 der Firma Veeco eingesetzt. Der Spitzenradius beträgt 12,5 $\mu \mathrm{m}$ und die verwendete Anpresskraft entspricht $10 \mathrm{mg}$.

- Es wurden Zugversuche bis zum Bruch der Probe in zwei Teile durchgeführt, um die mit Wasserstoff beladenen Proben, die von Rissen durchzogen sind, zur Beobachtung zu öffnen. Dazu wurde die Materialprüfmaschine Zwick 1474 der Firma Zwick/Roell eingesetzt. In dieser Arbeit wurde eine Prüfgeschwindigkeit von $1 \mu \mathrm{m} / \mathrm{s}$ verwendet.

\section{Finite-Elemente Simulationen}

Zur Unterstützung der experimentellen Ergebnisse wurden Simulationen mit einer FiniteElemente-Methode durchgeführt. Es werden die Spannungen simuliert, die durch einen Wasserstoffdruck in einem Riss in das umliegenden Material induziert werden. Dafür wurde das Programm COMSOL Multiphysics 5.2 verwendet. Alle Simulationen wurden mit der Hilfe von Niklas Teichmann durchgeführt. Es handelt sich um zweidimensionale Simulationen, in denen Eisen als linear elastisch angenommen wird. 


\section{Präparation und Charakterisierung der verwendeten Eisenproben \\ \begin{tabular}{l|l} 
Chemische Zusammensetzung der Proben & 4.1
\end{tabular}}

In dieser Arbeit wird Eisen mit verschiedenen Reinheitsgraden verwendet. Zunächst wurde der Effekt von Wasserstoff auf ein hochreines Eisen untersucht. Die hohe Reinheit soll es ermöglichen, den Mechanismus der Rissbildung im Detail zu verstehen. Danach wird Eisen mit einer geringeren Reinheit verwendet und untersucht, welche zusätzlichen Effekte auftreten. Zusätzlich wird über Kaltwalzen und anschließende Wärmebehandlung die Mikrostruktur des reinen Eisens variiert.

Das Eisen mit hoher Reinheit ist mehrfach umgeschmolzenes, entgastes Elektrolyteisen ${ }^{1}$. Die chemische Zusammensetzung wurde mittels Massenspektrometrie mit induktiv gekoppeltem Plasma (ICP-MS) gemessen². Bei dieser Messmethode wird ein zylinderförmiges Volumen der Probe in Abhängigkeit von der Tiefe analysiert, sodass ein Loch von $100 \mu \mathrm{m}$ Durchmesser und $22 \mu \mathrm{m}$ Endtiefe entsteht, wobei auf derselben Probe an zehn Stellen gemessen wird. Es zeigt sich bei der Vermessung einer Probe, dass keine signifikanten Schwankungen der Zusammensetzung innerhalb einer Probe auftreten. Die mittlere Zusammensetzung der reinen Eisenprobe ist in Tabelle 4.1 zusammengefasst, wobei bemerkt werden muss, dass nicht alle Elemente mit dieser Messmethode nachgewiesen werden können. Es kann zum Beispiel keine Aussage über den Kohlenstoffanteil getroffen werden.

\begin{tabular}{|l|c|c|c|c|c|c|}
\hline Element & $\mathrm{Mn}$ & $\mathrm{Cu}$ & $\mathrm{Cr}$ & $\mathrm{Co}$ & $\mathrm{Ge}$ & $\mathrm{Ni}$ \\
\hline Anteil $[\mathrm{ppm}]$ & $6.9 \pm 2.4$ & $7.4 \pm 2.7$ & $6.5 \pm 3.1$ & $4.8 \pm 2.2$ & $9.5 \pm 5.7$ & $3.2 \pm 3.9$ \\
\hline
\end{tabular}

Tabelle 4.1: Zusammensetzung der reinen Eisenprobe gemessen mit ICP-MS. Es sind alle Spurenelemente aufgeführt die mit mehr als $1 \mathrm{ppm}$ in der Probe nachgewiesen wurden. Die Elemente H, C, N, O und F können nicht mittels ICP-MS gemessen werden, da es zu Interferenzen mit Umgebungsatomen kommt.

Die Zusammensetzung der reinen Eisenprobe, siehe Tabelle 4.1, zeigt, dass es sich um sehr reines Eisen handelt, in dem die noch vorhandenen Spurenelemente Konzentrationen im

\footnotetext{
${ }^{1}$ Eisen wurde am Max-Planck-Institut für Eisenforschung in Düsseldorf hergestellt

${ }^{2}$ ICP-MS Messungen durchgeführt von Dr. Klaus Simon, Geochemie am Geowissenschaftliches Zentrum (GZG) an der Georg-August-Universität Göttingen
} 
maximal einstelligen ppm Bereich aufweisen. Als Vergleich für ein unreineres Material wurde ARMCO-Eisen verwendet, dieses hat eine Reinheit von 99,8\%, vergleiche Tabelle4.2. ARMCO-Eisen weist zum Beispiel etwa 100-mal so viel Mangan auf.

\begin{tabular}{|l|c|c|c|c|c|}
\hline Element & $\mathrm{C}$ & $\mathrm{Si}$ & $\mathrm{Mn}$ & $\mathrm{P}$ & $\mathrm{S}$ \\
\hline Anteil [at.\%] & 0,05 & Spuren & 0,06 & 0,009 & 0,005 \\
\hline
\end{tabular}

Tabelle 4.2: Typische Werte der Zusammensetzung von ARMCO-Eisen entnommen aus http: //www.chemie.de/lexikon/ARMCD-Eisen.html, aufgerufen am 26.07.2016

\section{Probenpräparation und resultierende Mikrostruktur der Proben}

Alle Proben wurden auf SiC Papier bis zu einer Körnung von 4000 (Korngröße $6 \mu \mathrm{m}$ ) nass geschliffen und in einem Vakuum-Ofen bei $\sim 10^{-7}$ mbar Hintergrunddruck ausgelagert. Die Wärmebehandlung erfolgte bei $880^{\circ} \mathrm{C}$ für 5 Stunden. Diese Temperatur liegt wenig unterhalb der Umwandlungstemperatur zum $\gamma$-Eisen $\left(911^{\circ} \mathrm{C}\right)$. Es sollte dadurch eine besonders niedrige Defektdichte und große Korngröße vorliegen. Abbildungen 4.1a und b
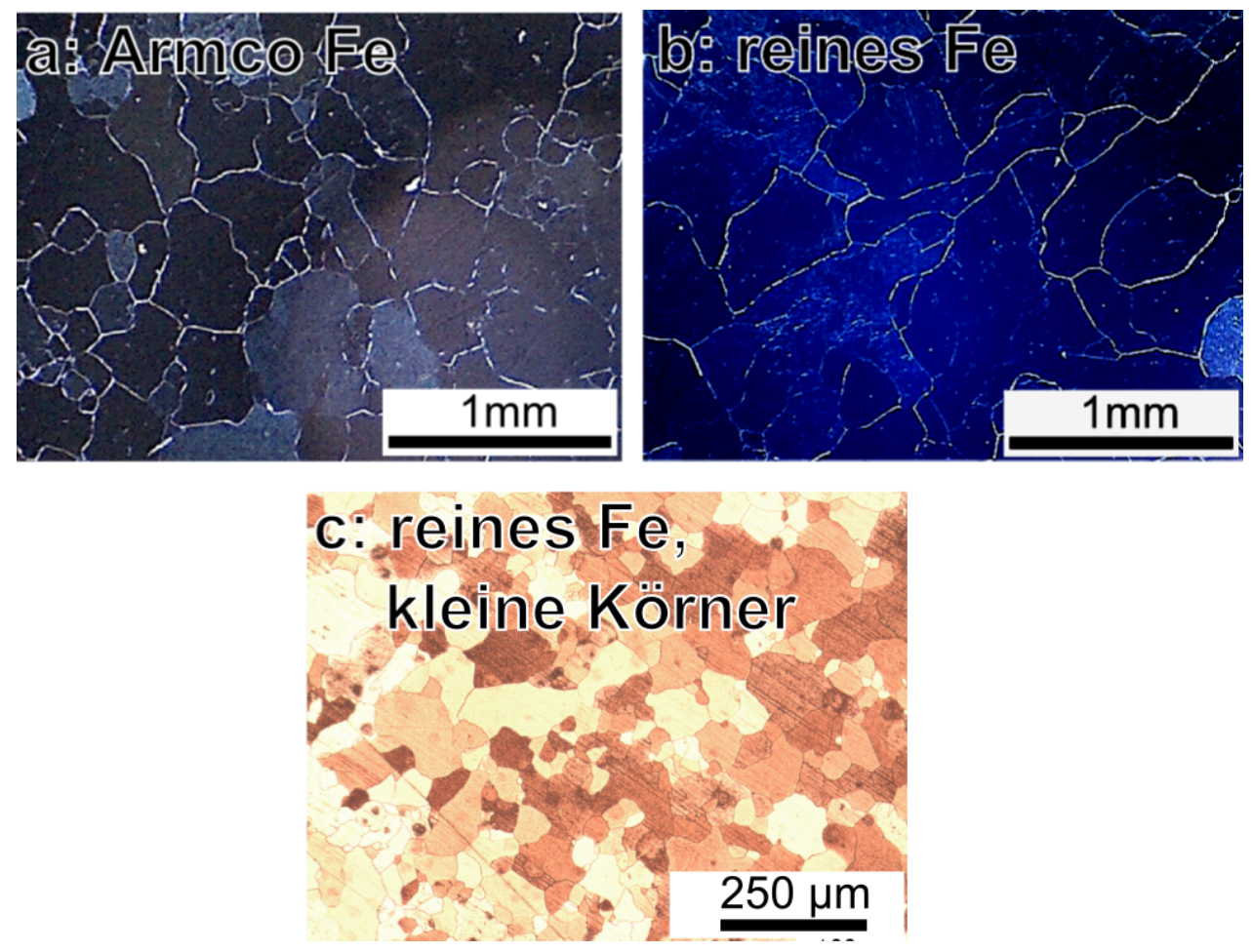

Abbildung 4.1: Lichtmikroskop-Bilder der Proben nach Präparation für die Wasserstoffbeladung: a) ARMCO-Eisen, ausgelagert 5 Stunden bei $880^{\circ} \mathrm{C}$, b) reines Eisen, ausgelagert 5 Stunden bei $880^{\circ} \mathrm{C}$, c) reines Eisen, gewalzt und 2 Stunden bei $600^{\circ} \mathrm{C}$ ausgelagert. 
zeigen lichtmikroskopische Aufnahmen der beiden verschiedenen Eisenproben nach dem Auslagern ( 5 Stunden bei $880^{\circ} \mathrm{C}$ ). Die Korngröße $d$ wurde aus den Mikroskopaufnahmen abgeschätzt und liegt bei:

$$
d_{\text {reinesFe }}=(0,48 \pm 0,28) \mathrm{mm} \quad d_{\mathrm{ARMCO}-\mathrm{Fe}}=(0,25 \pm 0,28) \mathrm{mm}
$$

Die Korngröße ist bei dem reinen Eisen etwa doppelt so groß wie bei ARMCO-Eisen. Dieses entspricht der Erwartung, da sich bei reineren Metallen größere Körner bilden. Die Größenordnung ist jedoch dieselbe, sodass ein unterschiedliches Verhalten aufgrund der Korngröße nicht zu erwarten ist. Um den Einfluss der Korngröße zu untersuchen, wurden reine Eisenproben hergestellt, die eine deutlich kleinere Korngröße aufweisen. Dafür wurden die Proben in mehreren Walzschritten auf $<80 \%$ ihrer ursprünglichen Dicke gewalzt. Zwischen den Walzschritten wurden die Proben jeweils um $90^{\circ}$ gedreht. Danach wurden die Proben 2 Stunden bei $600^{\circ} \mathrm{C}$ ausgelagert. Die Prozedur zur Korngrößenreduzierung wurde nach Ahmad et al. $\left[\mathrm{AKS}^{+} 14\right]$ durchgeführt. Der mittlere Korndurchmesser wurde aus Lichtmikroskop-Aufnahmen, vergleiche Abbildung 4.1c, bestimmt und liegt bei

$$
d_{\text {reinesFe,kleineKrner }}=(36 \pm 24) \mu \mathrm{m} .
$$

Die Korngröße konnte durch den beschriebenen Kaltwalz- und Auslagerungsprozess demnach um etwa eine Größenordnung reduziert werden. 


\section{\begin{tabular}{l|l} 
& \\
Ergebnisse & 5
\end{tabular} \begin{tabular}{l|l} 
Experimentelle Ergebnisse & 5.1
\end{tabular}}

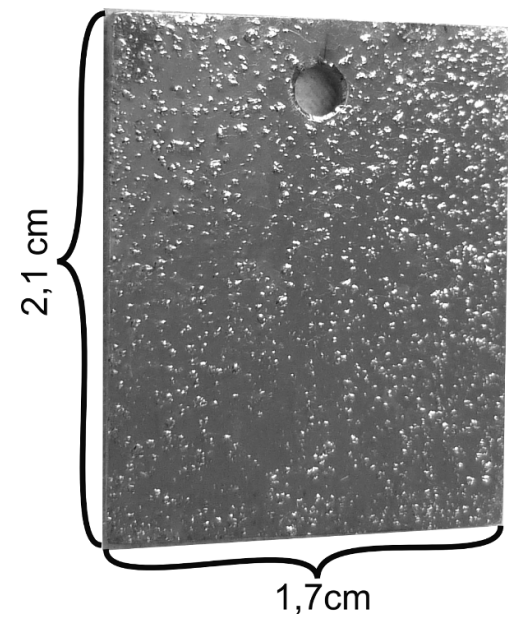

Abbildung 5.1: Fotografie einer Eisen-Probe nach Beladung. Das Loch oben in der Probe dient zur Aufhängung im Elektrolyten. Die Probenoberfläche ist mit Blistern übersät.

Die Messungen dieser Arbeit zielen darauf ab, die Riss- und Blisterentstehung durch Wasserstoff vollständig zu verstehen. Dabei werden die in Kapitel 3 beschriebenen Messmethoden verwendet. Die Ergebnisse sind wie folgt gegliedert:

- Zunächst erfolgt in den Abschnitten 5.1.1 bis 5.1.6 eine detaillierte Beschreibung der Betrachtung der Reineisen-Probe angefangen mit Fotografien bis hin zu TEM-Aufnahmen. Aus dieser Betrachtung können in Kapitel 6 Rückschlüsse auf die Initiierung und das Wachstum der Risse gezogen werden. Dabei wird zunächst immer das reine Eisen untersucht, das vor der Wasserstoffbeladung (5 Stunden bei $880^{\circ} \mathrm{C}$ ) ausgelagert wurde.

- In Abschnitt 5.1.7 werden die Unterschiede in der Rissbildung durch Variation des Reinheitsgrads und der Mikrostruktur des Eisens gezeigt. Es wird ARMCO-Eisen verwendet und das reine Eisen mit kleineren Körnern, wie es in Kapitel 4 beschrieben wurde.

- Abschließend wird die Triebkraft der Rissbildung, der gasförmige Wasserstoff in den Rissen, näher untersucht. Es wird der Wasserstoffdruck ermittelt, der in den Rissen herrscht und die Kinetik des Ausgasens von Wasserstoff aus der Probe untersucht.

\section{Erscheinungsbild der Proben nach der Wasserstoffbeladung}

Die Reineisen-Proben wurden, wie in Abschnitt 3.1 näher beschrieben, elektrochemisch mit Wasserstoff beladen. Nach dieser Beladung ist die Oberfläche mit Blistern übersät. 
Blister sind Aufwürfe von Probenmaterial auf der Oberfläche, die auf den ersten Blick wie Blasen aussehen, siehe dazu die Fotografie einer Eisenprobe nach der Beladung in Abbildung 5.1. Eine detaillierte Untersuchung ist mittels optischer Mikroskopaufnahmen verschiedener Vergrößerungen möglich, siehe Abbildungen 5.2 und 5.3. In Abbildung 5.2 ist eine Übersichtsaufnahme zu sehen. Zwischen den Blistern gibt es Bereiche, in denen eine unveränderte Probenoberfläche zu finden ist. Es liegt demnach keine vollständige Bedeckung mit Blistern vor. In Abbildung 5.3 ist eine höhere Vergrößerung der Probenoberfläche zu sehen. Bei dieser Vergrößerung ist zu erkennen, dass es sich nicht um runde Erhebungen handelt, sondern, dass die Blister an den Seiten Flanken aufweisen, die ein Plateau umgeben. Außerdem wird beobachtet, dass es häufig Blister gibt, auf denen wiederum kleinere Blister lokalisiert sind. Diese sind meist an den Flanken des großen unteren Blisters zu finden. Um die Form eines Blisters näher zu untersuchen und die Höhe zu messen, wurde von mehreren Blistern mit einem Profilometer ein Höhenprofil erstellt, indem jeweils ein Linienscan über die Mitte eines Blisters durchgeführt wurde. Die Auftragung von vier repräsentativen Höhenprofilen ist in Abbildung $5.4 \mathrm{zu}$ sehen. Die Höhe der Blister bewegt sich meist zwischen 2 und $7 \mu \mathrm{m}$, wie bei Blister 2 bis 4 in Abbildung 5.4 zu sehen. Einzelne Blister weisen Höhen von $15 \mu \mathrm{m}$ auf. Dies ist beispielhaft anhand des Blisters 1 in Abbildung 5.4 zu sehen. Der Durchmesser eines typischen Blisters ist etwa $100 \mu \mathrm{m}$, wie auch in den optischen Mikroskopaufnahmen zu sehen ist. Die Form der Blister besteht aus einem Plateau mit abgeschrägten Flanken am Rand des Blisters, dies ist besonders deutlich bei den Blistern 1, 2 und 4 in Abbildung $5.4 \mathrm{zu}$ sehen. Anhand des Blister 4 lässt sich erkennen, dass das Plateau bei einigen Blistern nicht parallel zu Oberfläche der Probe ist. Eine detaillierte Untersuchung der Blisterform wird anschließend mit einer REM-Untersuchung durchgeführt, siehe Abschnitt 5.1.2.

\section{\begin{tabular}{l|l} 
Rasterelektronenmikroskopische Untersuchung & 5.1 .2
\end{tabular}}

\section{Blister auf der Probenoberfläche}

Detailaufnahmen eines Blisters, die mit dem Rasterelektronenmikroskop angefertigt wurden, sind in den Abbildungen 5.5 und 5.6 zu sehen. Im Vergleich zu den optischen Mikroskopaufnahmen, wie in Abschnitt 5.1.1 gezeigt, können hier die Details der Flanken und des Plateaus näher betrachtet werden.

In Abbildung 5.5 ist ein typischer Blister zu sehen. Dieser erhebt sich mit einer deutlichen Grenze aus der ungestörten Probenoberfläche. Die Flanken bestehen aus feinen Stufen. Der Übergang zum flachen Plateau weist einen diskreten Übergang auf, was auch aus den Höhenprofilen verschiedener Blister, siehe Abbildung 5.4, hervorgeht. Die definierten Abgrenzungen sprechen für eine stark lokale Verformung. Lochfraß, der durch den sauren 


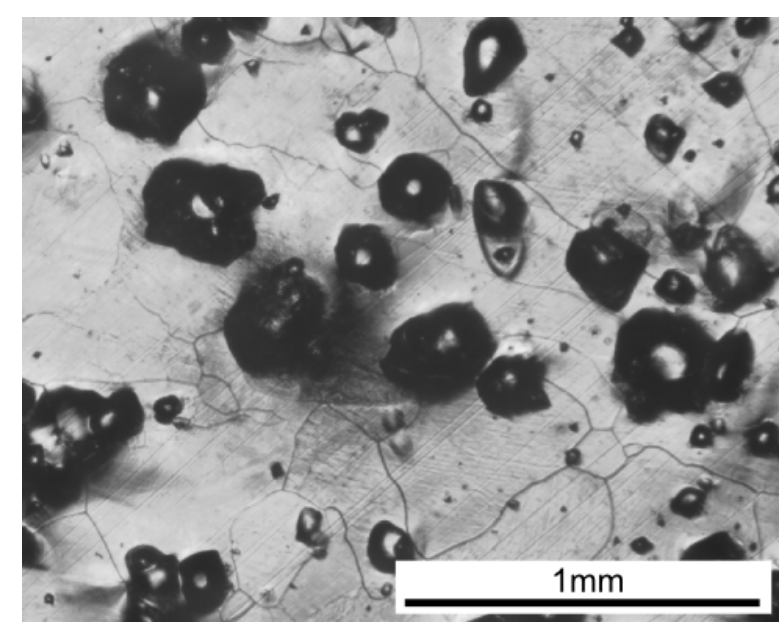

Abbildung 5.2: Lichtmikroskopaufnahme der Probenoberfläche mit 50-facher Vergrößerung nach Wasserstoffbeladung.

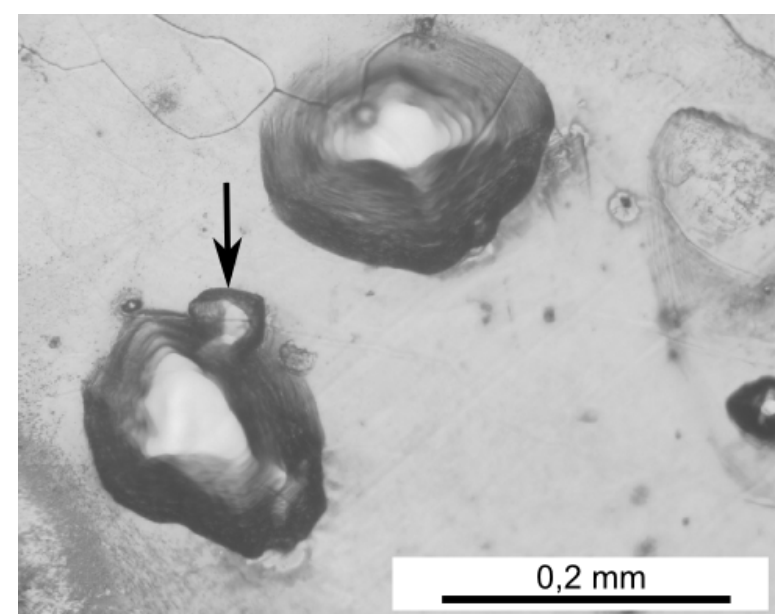

Abbildung 5.3: Lichtmikroskopaufnahme der Probenoberfläche mit 200-facher Vergrößerung nach Wasserstoffbeladung. Pfeil markiert kleinen Blister, der auf der Flanke eines größeren Blisters lokalisiert ist.

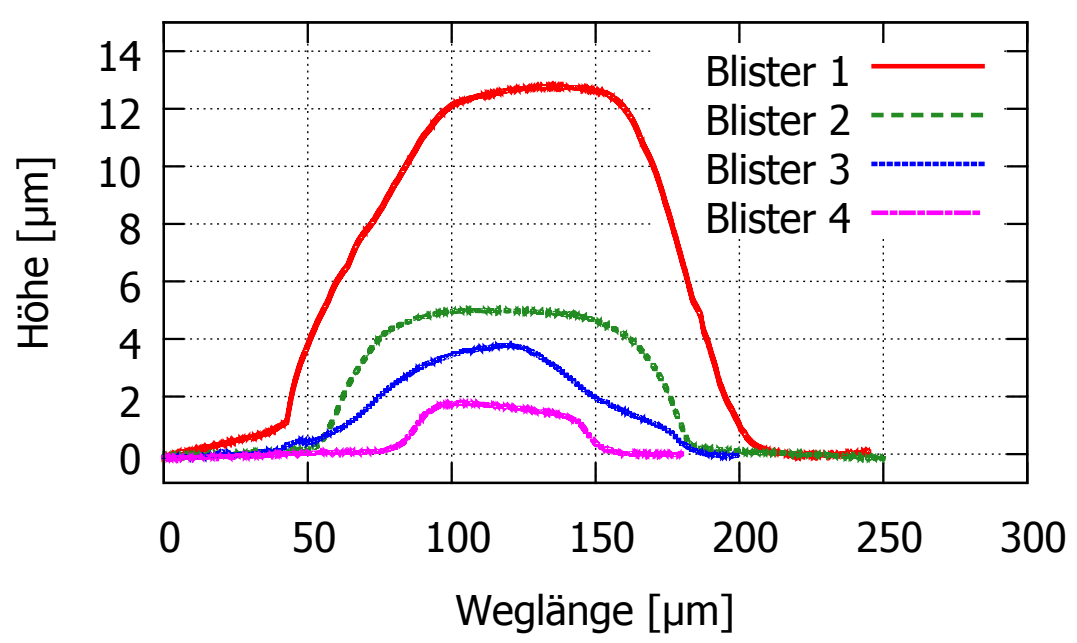

\begin{abstract}
Abbildung 5.4: Höhenprofil verschiedener Blister auf der Probenoberfläche. Es wurde jeweils ein Profil über die Mitte eines Blisters erstellt. Zu beobachten ist, dass alle Blister mit Ausnahme von Blister 3 seitlich Flanken und in der Mitte ein langes flaches Plateau aufweisen (man beachte hier die unterschiedliche Skalierung der Achsen). Eine typische Blisterhöhe beträgt 2 bis $7 \mu \mathrm{m}$, wobei auch Höhen bis zu $15 \mu \mathrm{m}$ gemessen wurden, diese werden durch Blister 1 repräsentiert.
\end{abstract}

Elektrolyten verursacht wird, ist hauptsächlich auf dem Plateau zu beobachten. Das deutet darauf hin, dass hier Versetzungen an der Oberfläche enden. In der Vergrößerung des Blisters in Abbildung 5.6 kann man die Stufen an den Flanken des Blisters gut erkennen. 


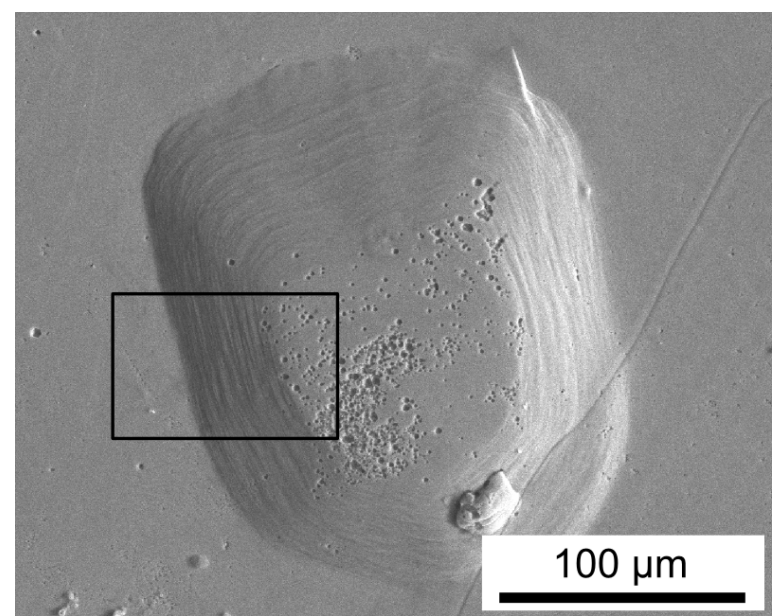

Abbildung 5.5: Eigene Ergebnisse veröffentlicht in $\left[\mathrm{TML}^{+} 16\right]$. REM-Aufnahme eines Blisters auf der Probenoberfläche nach Wasserstoffbeladung. Das Rechteck markiert den Ausschnitt, der in Abbildung 5.6 dargestellt ist. Man erkennt deutlich die Flanken und das Plateau, auf dem starker Lochfraß durch den sauren Elektrolyten zu sehen ist.

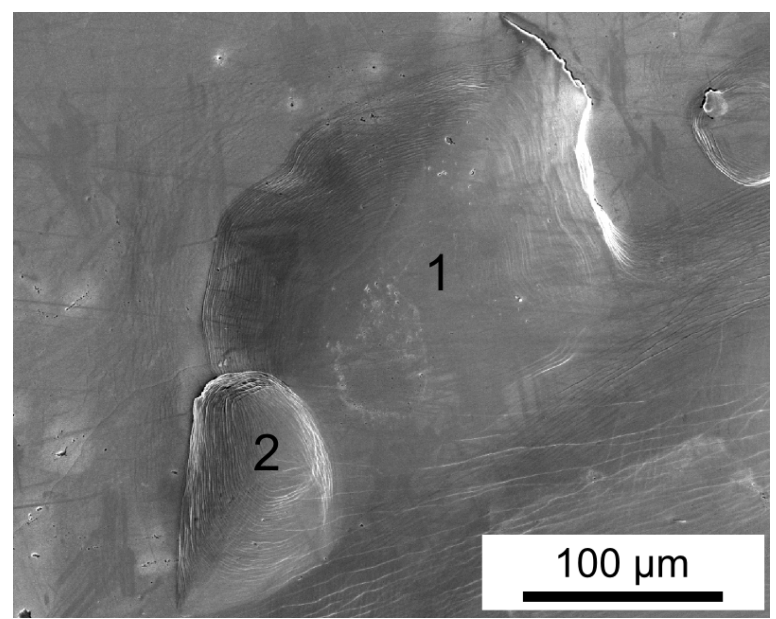

Abbildung 5.7: REM-Aufnahme eines Multiblisters, d.h. einem Blister auf dem weitere Blister gebildet wurden. Der ursprüngliche Blister ist mit 1 gekennzeichnet. Auf dessen Stufen ist der mit 2 gekennzeichnete deutlich kleinere Blister zu finden.

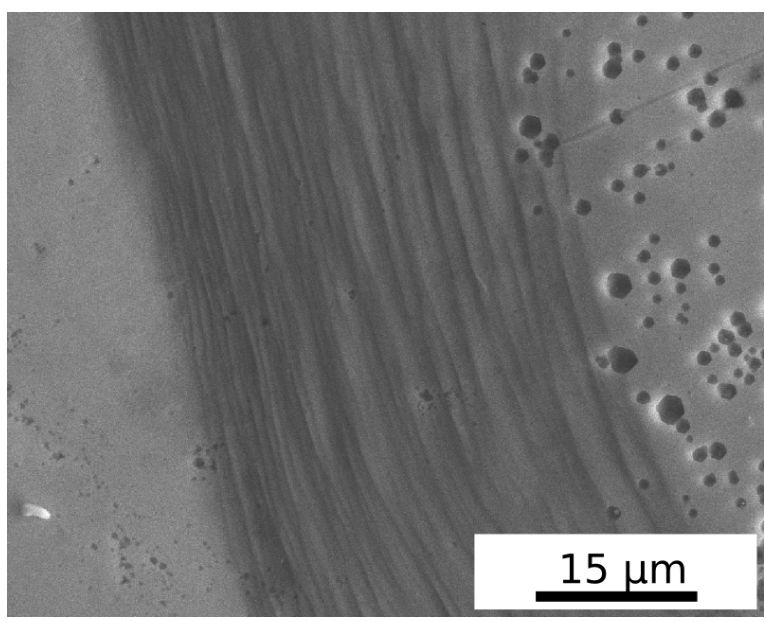

Abbildung 5.6: Vergrößerter Ausschnitt der REM-Aufnahme, Abbildung 5.5. Der Blister ist klar zum umliegenden Material (der ungestörten Probenoberfläche) abgegrenzt. Die Flanke des Blisters besteht aus feinen Stufen, die im Plateau enden. Dieser Blister hat etwa 30 Stufen auf einer Länge von $\sim 38 \mu \mathrm{m}$.

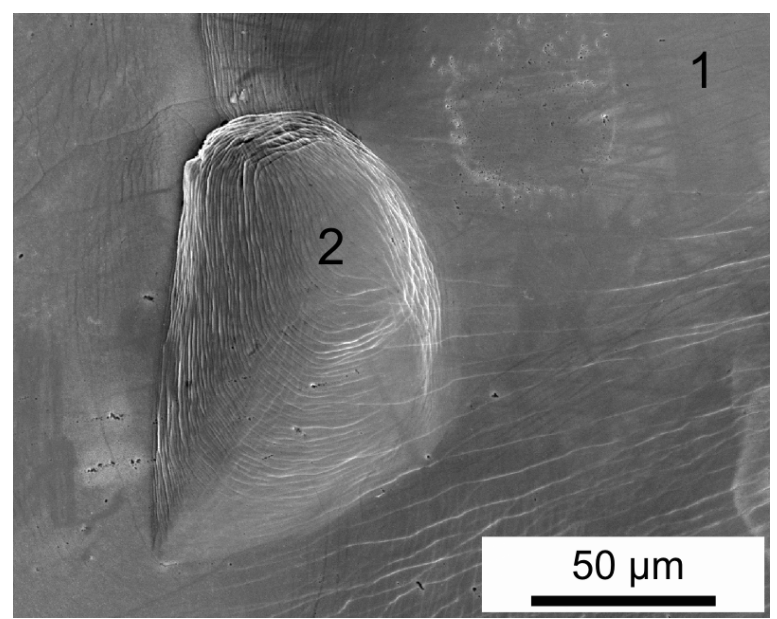

Abbildung 5.8: Vergrößerter Ausschnitt von Abbildung 5.7. Man sieht, dass die Stufen von Blister 1 auf dem Plateau und über die Stufen von Blister 2 verlaufen. 
Es sind $\sim 30$ Stufen auf einer Länge von $\sim 38 \mu \mathrm{m}$. Damit ergibt sich die Breite einer Stufe zu $\lesssim 1 \mu \mathrm{m}$ und deren Höhe ist $\sim 0,15 \mu \mathrm{m}$. Diese wird aus der Anzahl der Stufen und einer typischen Gesamthöhe von $5 \mu \mathrm{m}$ bestimmt.

In der lichtmikroskopischen Aufnahme in Abbildung 5.3 ist zu sehen, dass es zu der Bildung von kleineren Blistern auf größeren kommt. Ein solcher Komplex aus mehreren Blistern wird im Folgenden Multiblister genannt. Die kleinen Blister sind meist an den Flanken, das heißt auf den Stufen des primären Blisters zu finden, vergleiche Abbildung 5.3. In den Abbildungen 5.8 und 5.10 sind spezielle Merkmale, die an einzelnen Blistern und Multiblistern beobachtet werden, zu sehen. Diese sind repräsentativ und hilfreich, um den Entstehungsprozess zu verstehen. Eine REM-Aufnahme, die die Vergrößerung eines Multiblisters zeigt, ist in Abbildung 5.8 dargestellt. Hier kann man den genauen Verlauf der Stufen beider Blister erkennen. Der primäre Blister 1 hat breite Stufen am rechten unteren Bildrand und deutlich schmalere oben in der Mitte des Bildes. Auf dem Übergang dazwischen ist der kleine sekundäre Blister 2 zu finden. Dieser hat dieselbe typische Form eines Blisters, wie sie in Abbildung $5.5 \mathrm{zu}$ sehen ist. Zusätzlich zu den Stufen, die das Plateau von Blister 2 umgeben, kann man noch die Stufen von Blister 1 auf Blister 2 beobachten. Diese sind sowohl auf den Stufen senkrecht zu den Stufen von Blister 2 zu finden als auch auf dem Plateau.

Ein Blister, über den eine Korngrenze verläuft, ist in Abbildung 5.10 gezeigt. Die Stufen

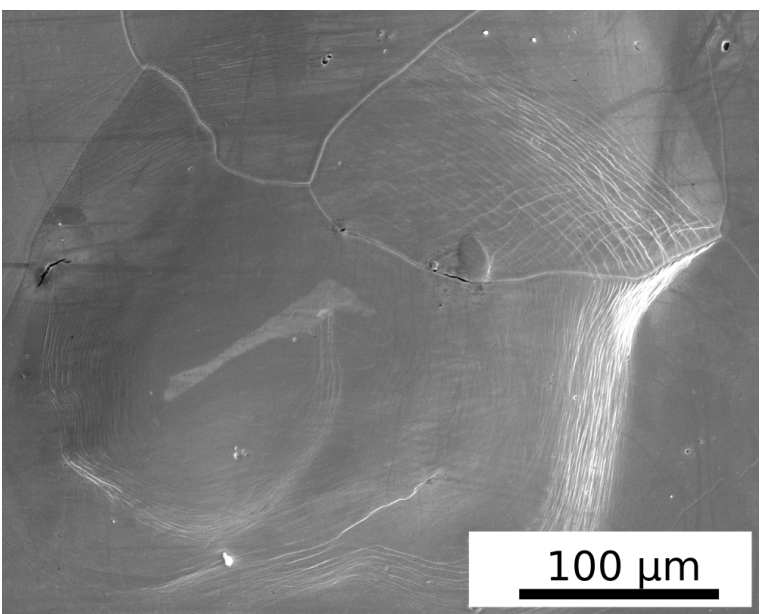

Abbildung 5.9: REM-Aufnahme eines Blisters, über den eine Korngrenze verläuft. Der Verlauf der Stufen ist vergrößert in Abbildung $5.10 \mathrm{zu}$ sehen.

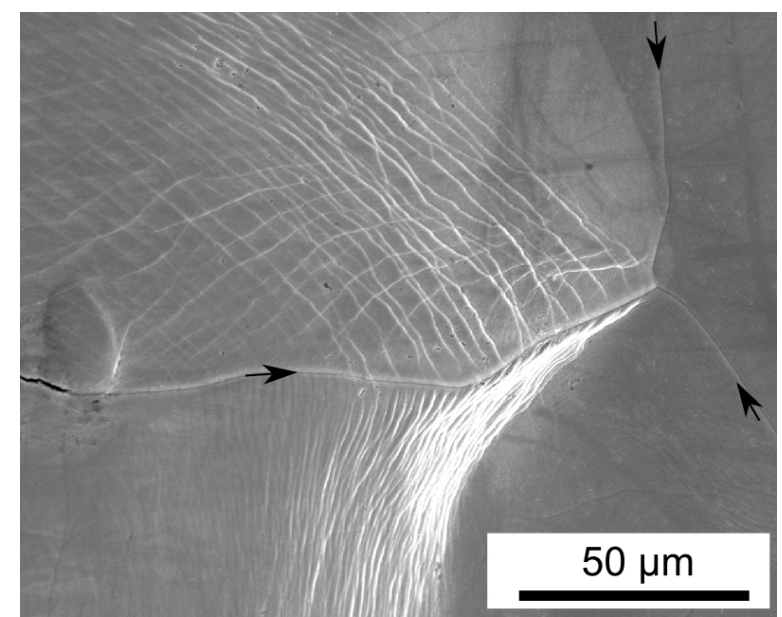

Abbildung 5.10: REM-Aufnahme der Stufen eines Blisters, über den eine Korngrenze verläuft. Die Korngrenzen sind mit Pfeilen markiert. Die Stufen wechseln an der waagerechten Korngrenze ihre Richtung. Der Blister endet an dem Tripelpunkt der drei Korngrenzen. 
verlaufen über die waagerechte Korngrenze. Über der Korngrenze, also im oberen Korn haben die Stufen einen höheren Abstand als im unteren Korn. Des Weiteren wechseln die Stufen an der Korngrenze ihre Richtung. Dies zeigt, dass die Blisterentstehung kristallographisch beeinflusst wird. In Abbildung 5.10 sind noch zwei weitere etwa senkrecht verlaufende Korngrenzen zu sehen, sodass insgesamt drei Körner abgebildet sind. Am Tripelpunkt der drei Korngrenzen enden die Stufen des Blisters. Hier wurde das Wachstum des Blisters gestoppt.

\section{Risse im Querschnitt der Probe}

In den vorherigen Abschnitten wurden Blister auf der Probenoberfläche beobachtet. Um nun zu ergründen, wie die Blister entstehen, wurden von verschiedenen Proben Querschliffe nach der Wasserstoffbeladung präpariert. Außerdem soll die Schädigung durch den Wasserstoff im Inneren der Probe analysiert werden.

In den Abbildungen 5.11 und 5.12 sind Querschliffe mit verschiedenen Vergrößerungen abgebildet. Einen Überblick über den gesamten Querschnitt einer 1,5 mm dicken Probe nach Wasserstoffbeladung ist in Abbildung 5.11 zu sehen. Es liegt eine deutliche Schädigung des Materials durch Risse über die gesamte Tiefe der Probe vor. Die Risse haben eine Länge

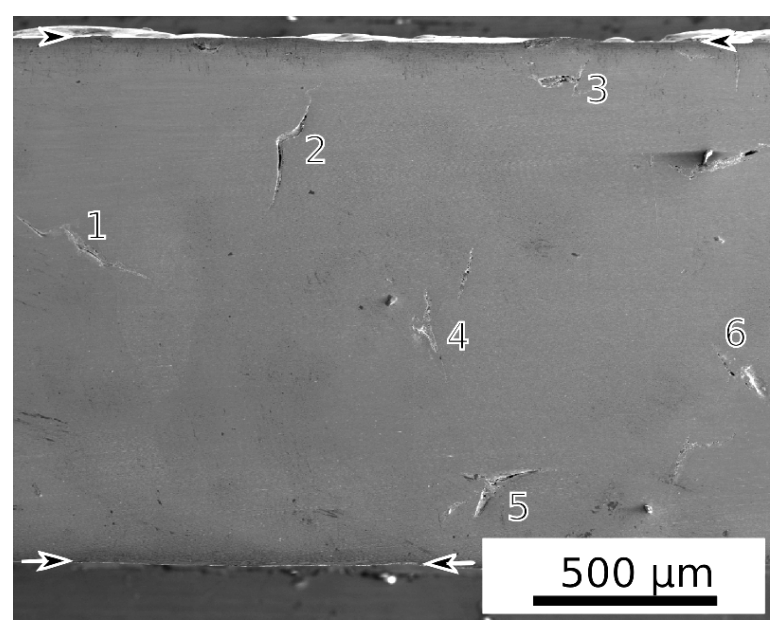

Abbildung 5.11: REM-Aufnahme des Querschnitts einer Probe. Am oberen und unteren Bildrand ist die Oberfläche der Probe mit Pfeilen gekennzeichnet. Es sind sechs Risse zu sehen, die sich über die gesamte Tiefe der Probe verteilen.

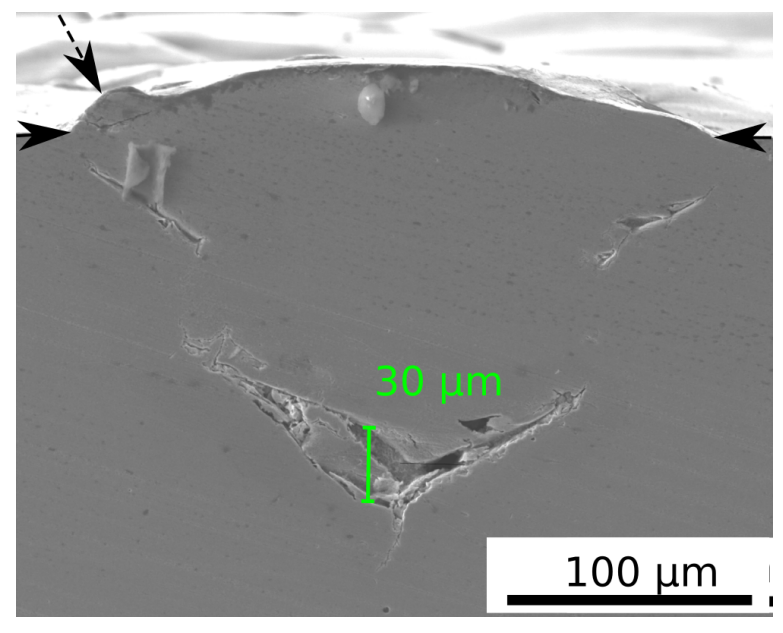

Abbildung 5.12: REM-Aufnahme des Querschnitts eines Multiblister. Die Probenoberfläche ist markiert mit zwei Pfeilen. Sie zeigt eine langgezogene Wölbung und darauf eine zweite kleinere Wölbung, die mit einem gestrichelten Pfeil markiert ist. Der Riss bzw. die drei Risse verursachen einen Multiblister auf der Oberfläche. Die Öffnung des Risses beträgt etwa $30 \mu \mathrm{m}$ und ist grün eingezeichnet. 
von bis zu $500 \mu \mathrm{m}$. An der breitesten Stelle sind die Risse bis zu $30 \mu \mathrm{m}$ geöffnet, vergleiche Abbildung 5.12. Die Orientierung der Risse im Inneren der Probe richtet sich nicht parallel zur Oberfläche der Probe aus, wobei das nicht für Risse nahe der Oberfläche gilt. Das wird später im Zusammenhang mit Abbildung 5.32 erläutert. Diese nicht vorhandene Ausrichtung der Risse im Inneren der Probe, ist zu erwarten, da eine polykristalline Probe ohne Vorzugsorientierung verwendet wurde. Die Risse verlaufen oft nicht gerade, sondern weisen Richtungswechsel auf, wie zum Beispiel Riss 1 und 2 in Abbildung 5.11. Ebenso werden oft Dreifach-Risse mit einem Tripelpunkt beobachtet, zum Beispiel Riss 4 und 5 . Ist ein Riss nahe der Oberfläche, wie es in Abbildung $5.12 \mathrm{zu}$ sehen ist, wird Probenmaterial in Richtung Oberfläche verschoben und auf der Oberfläche wird ein Blister erzeugt. Dies ist in Abbildung 5.12 an der Wölbung der Oberfläche zu erkennen. Ein typischer Riss, der mit einem Blister zusammenhängt, befindet sich circa $100 \mu \mathrm{m}$ unter der Oberfläche. Oft sind solche Risse parallel zur Oberfläche orientiert, da in dieser Geometrie das Material einfacher zur Oberfläche verschoben werden kann. In Abbildung 5.12 sind oberhalb des großen Risses zwei kleinere Risse zu sehen. Oberhalb des linken kleinen Risses ist eine deutliche Wölbung der Oberfläche zu erkennen (Wölbung markiert mit einem gestrichelten Pfeil), die sich auf der Flanke der Wölbung des großen Blisters befindet. Im Vergleich mit der Aufsicht auf einen Multiblister, wie in Abbildung $5.8 \mathrm{zu}$ sehen, handelt es sich bei den kleineren Rissen um sekundäre Risse, die einen Multiblister verursachen. Die Entstehung von primären und sekundären Rissen wird in Abschnitt 6.1 detailliert diskutiert.

\section{Oberfläche der Risse freigelegt durch Zugbelastung bis zum Bruch}

Nachdem durch die Wasserstoffbeladung Risse im Inneren der Probe entstanden sind, wie in Abbildung 5.11 dargestellt ist, sollte die Rissfläche näher untersucht werden. Dafür wurde mit der Probe nach der Beladung ein Zugversuch bis zum Bruch der Probe in zwei Teile durchgeführt. Die dabei entstandenen Spannungs-Dehnungs-Diagramme werden in Abschnitt 5.1.11 vorgestellt. Die Bruchfläche einer Probe nach Wasserstoffbeladung ist in Abbildung $5.13 \mathrm{zu}$ sehen. Die Breite der Bruchfläche entspricht etwa der ursprünglichen Probendicke von 1,5 mm. An der Probenoberfläche, die in Abbildung 5.13 weiß gestrichelt gekennzeichnet ist, ist zu erkennen, dass eine leichte Einschnürung der Oberfläche vorliegt. Diese ist vor allem oben in Abbildung 5.13 gut zu sehen. Die aufgrund des Zugversuches aufgetretene Einschnürung deutet auf einen teilweise duktilen Bruch durch die Zugbelastung hin. Man findet ebenfalls typische Merkmale von duktilem Bruch an hohen Teilen der Probe, exemplarisch gekennzeichnet mit grünen Pfeilen. Diese duktilen Merkmale sind in Abbildung 5.14 in einer Detailaufnahme zu sehen. In der Abbildung ist sogenannte Microvoid-Coalescence zu beobachten, vergleiche Abschnitt 2.2.2. Da die duktilen Bereiche die höchsten Teile der Bruchfläche darstellen, handelt es sich um den duktilen Bruch der Probe durch den Zugversuch, wie es auch schon die Einschnürung 


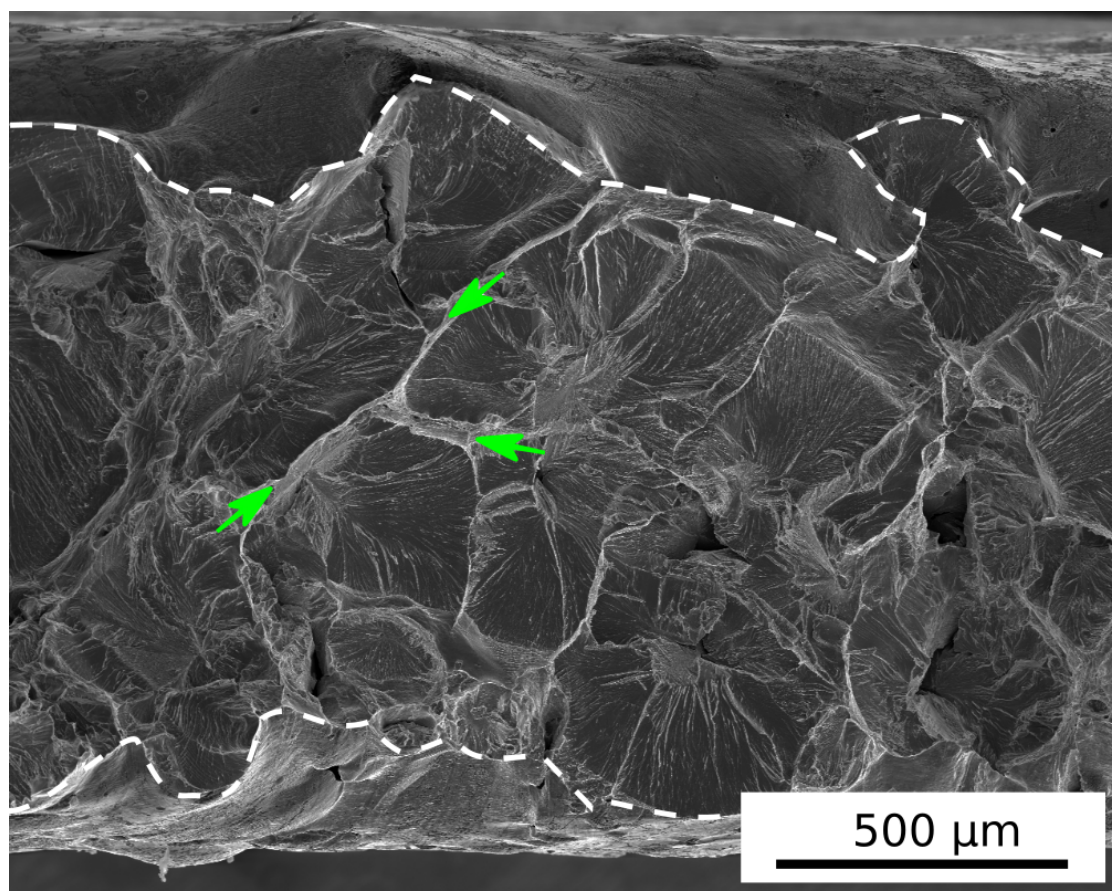

Abbildung 5.13: REM-Aufnahme der Bruchfläche einer mit Wasserstoff beladenen Probe nach Zugversuch. Die Bruchfläche sieht größtenteils spröde aus. Die Probenoberfläche ist mit einer weiß gestrichelten Linie markiert. An der Oberfläche der Probe kann man eine leichte Einschnürung erkennen (vor allem oben im Bild erkennbar). Duktile Kanten zwischen den spröden Flächen sind exemplarisch mit Pfeilen markiert.

vermuten ließ. Der Bruch durch den Zugversuch ist für die Untersuchung der Rissbildung durch Wasserstoff nicht relevant. Somit werden die duktilen Merkmale durch den Bruch nicht näher betrachtet.

Die spröden Bereiche zwischen den hohen duktilen Kanten stellen die Bruchflächen der geöffneten Risse dar, die durch die Wasserstoffbeladung schon vor dem Zugversuch in der Probe vorhanden waren. Diese sollen im Folgenden untersucht werden. Einen detaillierteren Blick auf eine Rissfläche und dessen Gegenstück auf dem zweiten Teil der auseinander gebrochenen Probe ist in den Abbildungen 5.15 und 5.16 gezeigt. In beiden Bildern beobachtet man eine konzentrische Geometrie. Unter Kippen der Probe im REM stellt man fest, dass der gezeigte Bereich in beiden Bildern nach außen höher wird. Die Risse sind, wie in Abbildung 5.11 gezeigt wurde, stark geöffnet. Dadurch kommt die Form beider Rissflächen, die nach außen höher werden, zustande. Im Zentrum der Bilder, Abbildung 5.15 und 5.16, befindet sich jeweils ein Loch mit einem Durchmesser von wenigen Mikrometern und in Abbildung 5.15 befindet sich ein Partikel innerhalb dieses Loches. Aufgrund der konzentrischen Geometrie um diese Partikel, kann davon ausgegangen werden, dass es sich hierbei um den Ursprung des Risses handelt. Die Risse breiten sich also nach der Initiierung nahezu konzentrisch um die Partikel aus. Besonders zu bemerken ist, dass das Loch 


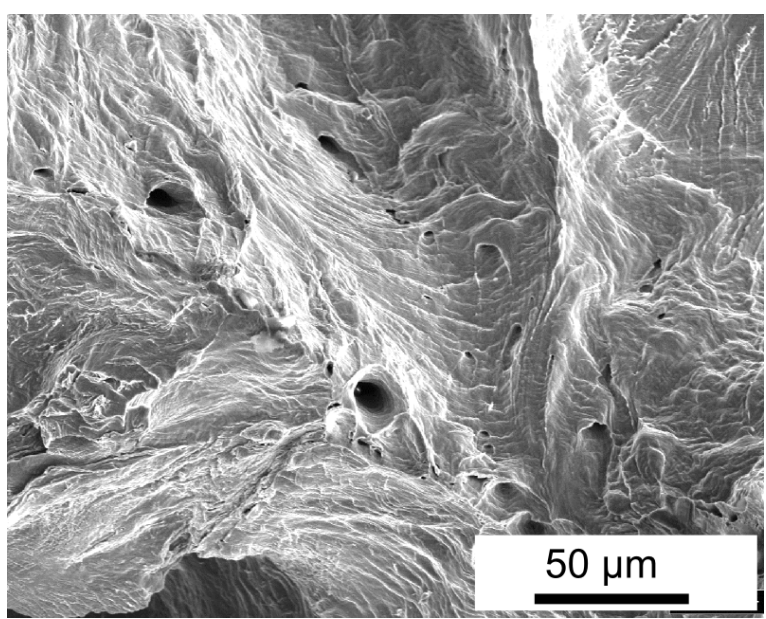

Abbildung 5.14: REM-Aufnahme, die eine Vergrößerung in einen duktilen Bereich darstellt. Duktile Merkmale sind speziell in hohen Bereichen zu finden, d.h. sie sind beim finalen Bruch der Probe durch den Zugversuch entstanden.

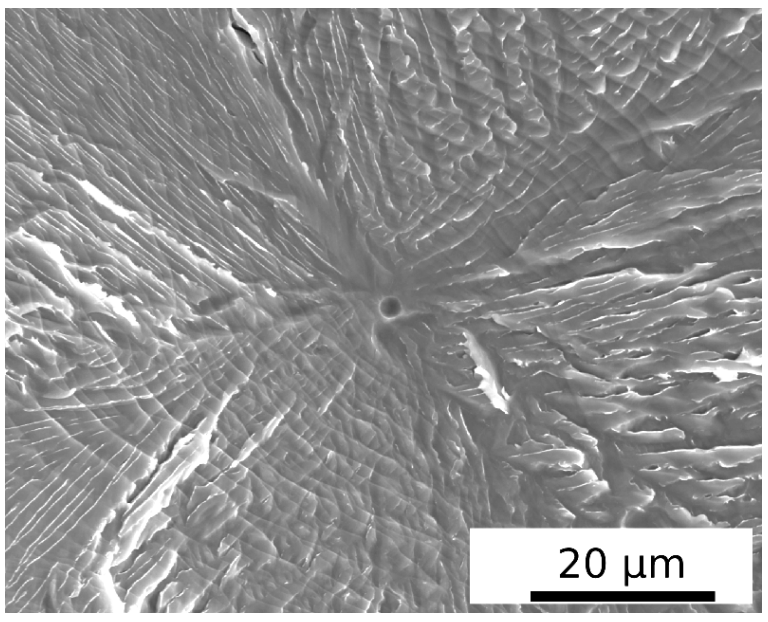

Abbildung 5.16: Eigene Ergebnisse veröffentlicht in $\left[\mathrm{TML}^{+} 16\right]$. Gegenstück zu Abbildung 5.15. Im Zentrum von konzentrisch angeordneten spröden Bruchstrukturen befindet sich ein rundes Loch von wenigen Mikrometern Durchmesser.

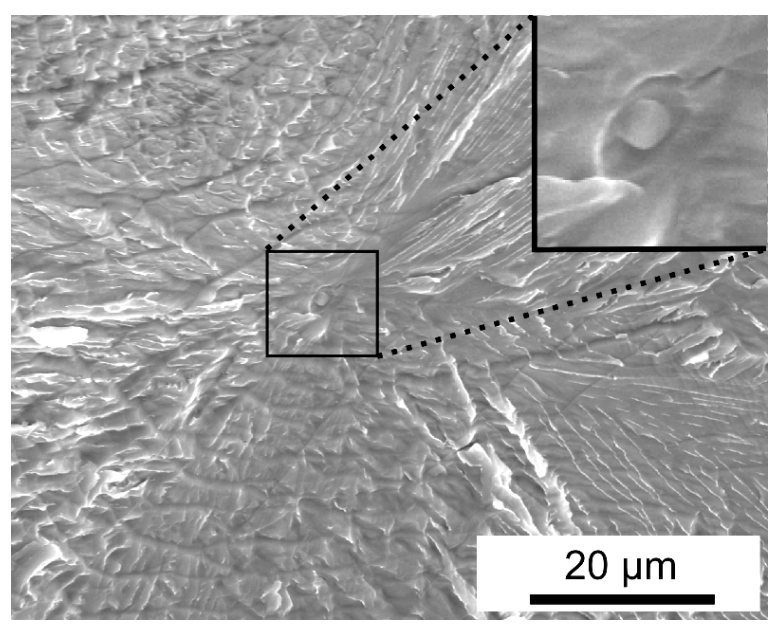

Abbildung 5.15: Eigene Ergebnisse veröffentlicht in $\left[\mathrm{TML}^{+} 16\right]$. REM-Aufnahme, die eine Vergrößerung eines spröden Bereiches darstellt. Man erkennt in der Mitte des Bildes ein Loch mit Einschluss darin. Ein Einschluss wird in Abbildung 5.18 näher untersucht.

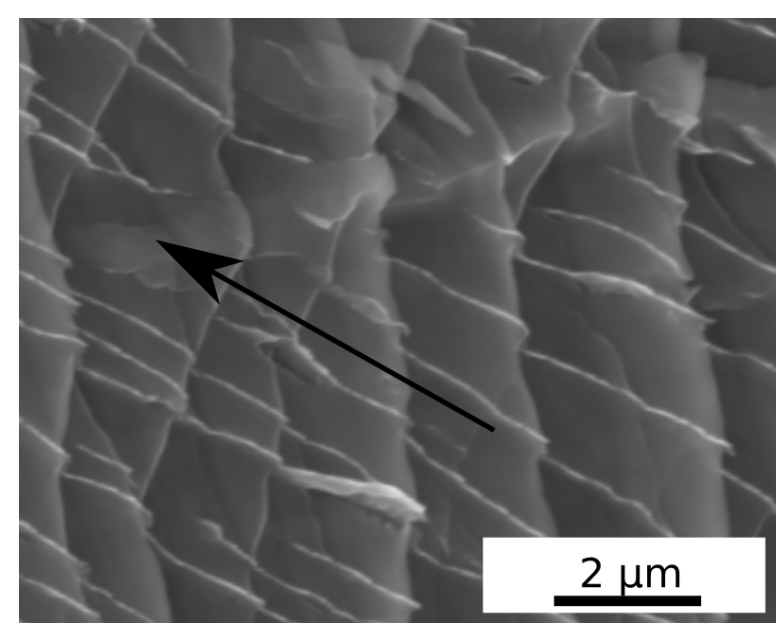

Abbildung 5.17: REM-Nahaufnahme der Rissfläche. Die Richtung der Rissausbreitung ist per Pfeil markiert. Man sieht Stufen senkrecht und Linien auf den Stufen parallel zur Rissausbreitung. 
um den Partikel deutlich größer ist als der Partikel selbst, wie in Abbildung 5.15 zu sehen ist. Dies deutet darauf hin, dass sich zunächst der Partikel von der umliegenden Matrix gelöst hat und durch den entstandenen Hohlraum der Ausgangspunkt eines Risses gebildet wurde. Um das Loch finden sich nahezu kreisförmig angeordnete Stufen, die zu der oben beschriebenen Form der Rissfläche als Tal führen. Des Weiteren gibt es linienförmige Merkmale, im Folgenden einfach Linien genannt, die vom Zentrum nach außen verlaufen. Beides ist in einer Vergrößerung der Stufen in Abbildung 5.17 im Detail zu sehen. Man kann eine Breite der Stufen von circa $1 \mu \mathrm{m}$ beobachten. Dies entspricht der Breite, die auch für die Stufen an den Flanken eines Blisters aus Abbildung 5.6 abgeschätzt wurde. Die Stufen sind in etwa senkrecht zur Ausbreitungsrichtung des Risses orientiert, was die Interpretation nahelegt, dass es sich hierbei um Haltepunkte während des Risswachstums handelt. Parallel zur Rissausbreitungsrichtung sind in Abbildung 5.17 feine weiße Linien zu erkennen, die über die Stufen hinweg beobachtet werden. Solche Linien werden oft im Zusammenhang mit Wasserstoff-induzierter Rissbildung beobachtet. Der zu Grunde liegende Bruchmechanismus wird Quasi-Spaltbruch genannt. Dieser Mechanismus ist in Abschnitt 2.2.2 erklärt. Die weißen Linien sind bei genauer Betrachtung im REM elektronentransparent und deuten somit auf eine lokal starke duktile Verformung hin. Der teilweise duktile Charakter des Bruches wird in Abschnitt 6.7 diskutiert.

\section{EDX-Untersuchung der Einschlüsse im REM $\mid 5.1 .3$}

Die Partikel, an denen die Risse initiiert werden, wie in Abbildung 5.15 gezeigt, wurden mittels EDX chemisch analysiert. An verschiedenen Partikeln, die oft im Zentrum eines Risses zu finden waren, wurden EDX-Punktscans durchgeführt. Die Partikel wurden nicht nur im Zentrum von Rissen, sondern auch in den Mulden der Microvoid-Coalescence, die durch den Bruch während des Zugversuchs zustande kommen, und sehr selten auf Rissflächen nicht im Zentrum eines Risses beobachtet.

Um eine generelle Verunreinigung der Probe auszuschließen, wurde jeweils in geringer Entfernung zum Partikel ein weiterer Punktscan aufgenommen, der die Zusammensetzung des umliegenden Materials ermittelt. In Abbildung 5.18 ist die EDX-Untersuchung an einem exemplarischen Partikel, von dem ein Riss ausgeht, gezeigt. Es sind ein Punktscan auf dem Partikel und ein Hintergrundscan in etwa $20 \mu \mathrm{m}$ Entfernung aufgetragen. Die genauen Positionen, an denen die Punktscans durchgeführt wurden, können aus dem REM-Bild in Abbildung 5.18 entnommen werden. Der Hintergrund-Scan zeigt, dass es sich bei dem Material der Matrix ausschließlich um Eisen handelt, wobei eine minimale Kontamination durch Kohlenstoff gemessen wird, die üblicherweise bei EDX-Messungen auftritt. Auf dem Partikel ist das stärkste gemessene Signal ebenfalls Eisen zuzuordnen, da die umgebende Eisen-Matrix mit gemessen wird. Auf dem Einschluss werden auch einige andere Elemente 


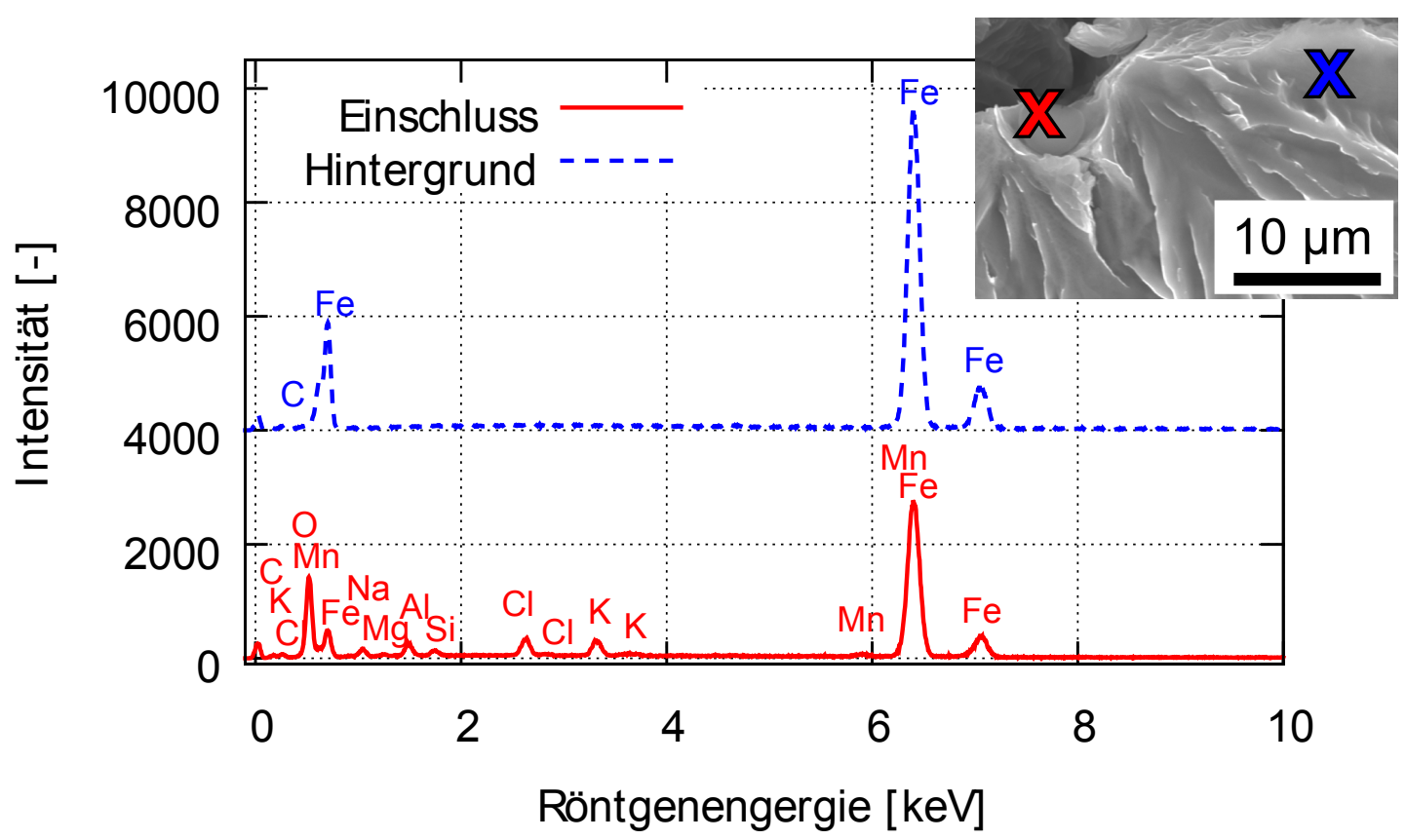

\begin{abstract}
Abbildung 5.18: Eigene Ergebnisse veröffentlicht in $\left[\mathrm{TML}^{+} 16\right]$. EDX-Punktscans an und neben einem Einschluss. Die REM-Aufnahme, die oben rechts im Bild zu sehen ist, zeigt die Positionen, an denen EDX-Punktscans durchgeführt wurden. Aus der Auftragung ist zu entnehmen, dass an dem Einschluss verschiedene Elemente nachgewiesen werden, die im Hintergrund-Scan nicht gemessen wurden.
\end{abstract}

nachgewiesen. Um einen Überblick zu erlangen, ist in Abbildung 5.19, eine Übersicht über EDX-Punktscans an mehreren Einschlüssen zu sehen. Über der Auftragung ist eine Zuordnung der Peaks zu den verschiedenen Elementen dargestellt. Ist ein Element schwarz gedruckt wird es an fast jedem Einschluss nachgewiesen. Die Elemente, die grau markiert sind, sind hingegen nur an einzelnen Einschlüssen detektiert worden. Häufig detektierte Elemente sind Sauerstoff, Mangan, Aluminium und Silizium. Bei den Einschlüssen handelt es sich somit um Oxide, genauer um Mangan-, Aluminium- und Siliziumoxid. Titan und Schwefel werden an keinem Einschluss nachgewiesen, was im Vergleich mit der Literatur von Relevanz ist und in Abschnitt 6.2 diskutiert wird.

\title{
\begin{tabular}{l|l} 
Kristallographische Orientierung mittels EBSD im REM & 5.1 .4
\end{tabular}
}

Alle EBSD Messungen wurden in Kooperation mit May L. Martin durchgeführt. Es wurde in Abschnitt 5.1.2 beobachtet, dass die Rissausbreitung kristallographisch beeinflusst wird. Deshalb wird in diesem Abschnitt die kristallographische Orientierung der Rissflächen bestimmt. Außerdem kann man mit EBSD die plastische Verformung um die Risse darstellen. 


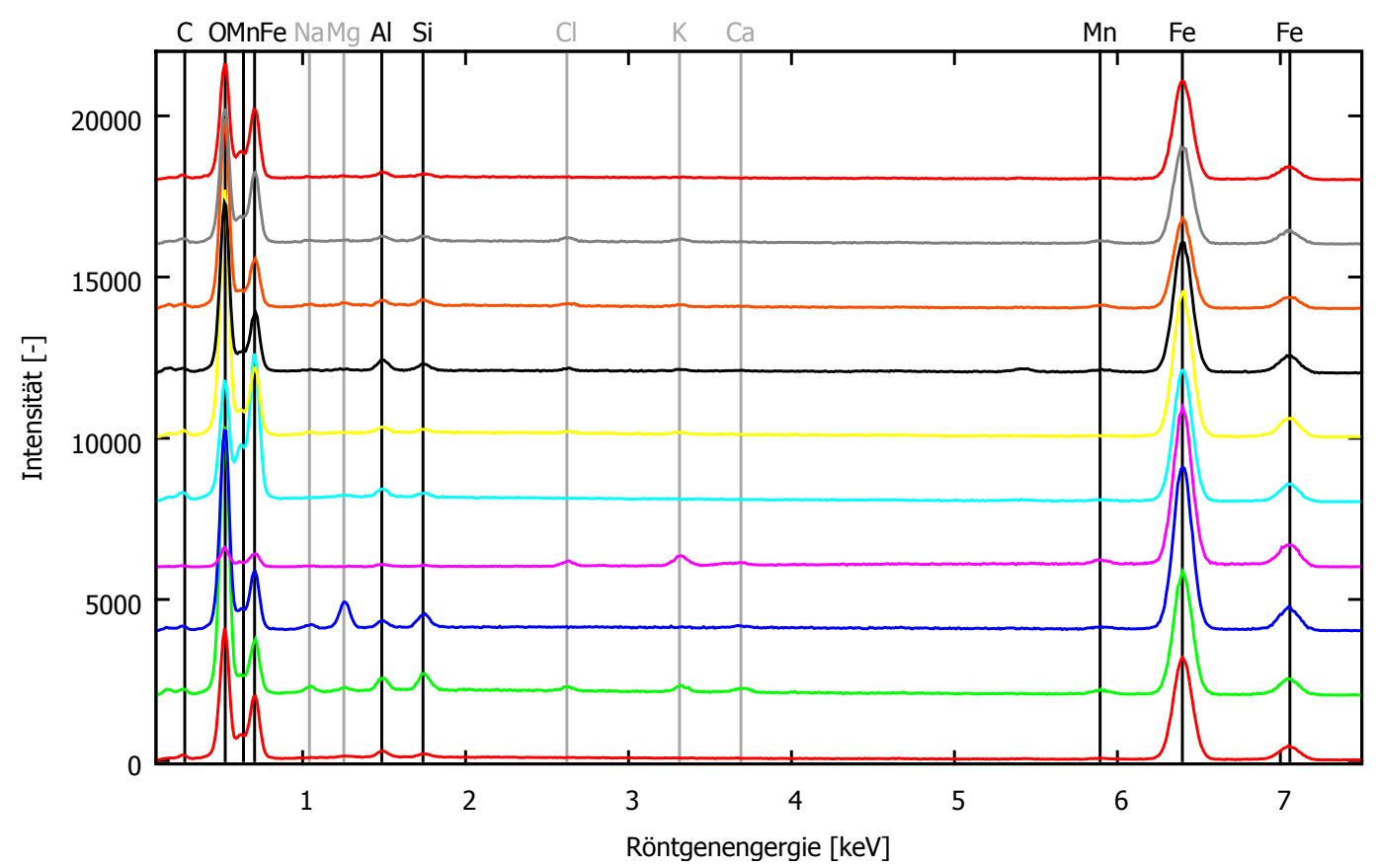

Abbildung 5.19: EDX-Punktscans an verschiedenen Einschlüssen. Die Elemente, die schwarz gekennzeichnet sind, werden an jedem Einschluss nachgewiesen, die grau gekennzeichneten nur an einigen. Man erkennt, dass es sich mit hoher Wahrscheinlichkeit um Oxide handelt, da das Sauerstoffsignal hoch ist, außerdem werden Mangan, Aluminium und Silizium nachgewiesen, was eine Mischung aus Mangan-, Aluminium- und Siliziumoxid nahelegt. Schwefel oder Titan werden hingegen nicht nachgewiesen.

\section{Deformationszone um Risse}

In Abbildung 5.20 ist eine EBSD-Messung zu sehen, die an einer Querschlifffläche einer mit Wasserstoff beladenen Probe durchgeführt wurde. Wie in Abschnitt 3.2 beschrieben, wird zur Darstellung eine Euler-Karte verwendet. Der Umriss des Risses ist mit einer gestrichelten Linie markiert. Innerhalb des Risses wird größtenteils kein Signal gemessen, diese Punkte sind schwarz, ebenfalls werden beliebige Orientierungen gemessen, diese werden oft als Konfetti-Punkte bezeichnet und erlauben keine Aussage bezüglich der Orientierung. Rechts im Bild ist ein anders orientiertes Korn zu sehen als im Rest des Bildes, was an einer deutlich unterschiedlichen Farbe zu erkennen ist. In direkter Nähe $(\lesssim 5 \mu \mathrm{m})$ um den Riss ist eine starke farbliche Veränderung, das heißt eine deutliche Kornrotation, zu erkennen. Des Weiteren ist links des Risses auch über die Rissspitze hinaus eine Aufhellung zu erkennen. Um die Kornrotation in diesem Bereich näher zu betrachten, ist ein Linien-Scan senkrecht zum Riss über dessen Rissspitze angefertigt worden. Dieser ist mit einem Pfeil in der EBSD-Messung gekennzeichnet und das Resultat ist in Abbildung 5.21 abgebildet. Aufgetragen ist hier die Rotation des jeweiligen Punktes im Bezug zu dem Anfangspunkt des Linien-Scans. Bis zum Riss ist auf einer Länge von ungefähr $30 \mu \mathrm{m}$ ein 


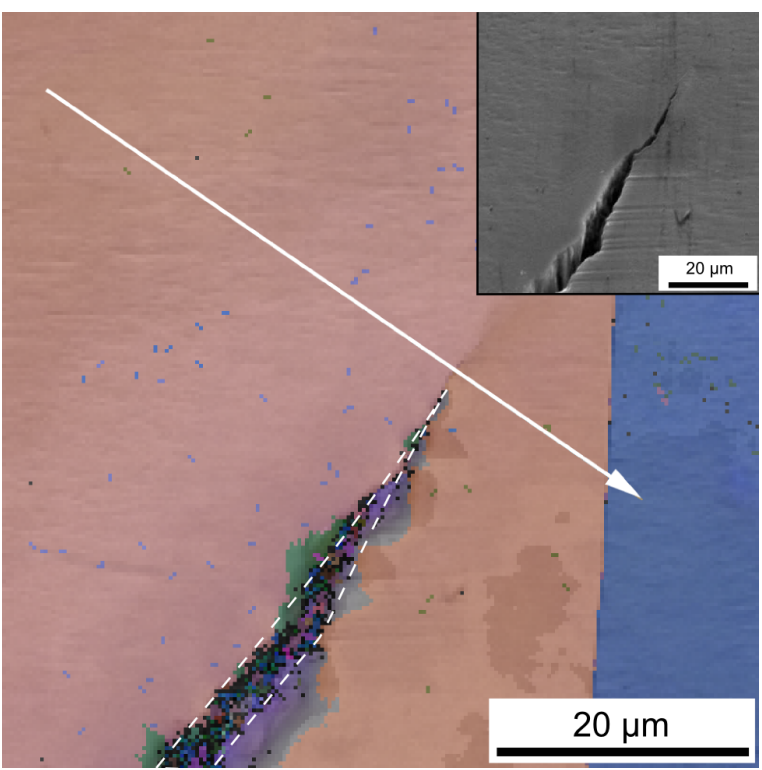

Abbildung 5.20: Bild erstellt in Zusammenarbeit mit May L. Martin, veröffentlicht in $\left[\mathrm{TML}^{+}\right.$16]. EBSD Euler Karte. Rechts oben ist das REM-Bild der gleichen Stelle abgebildet. Rechts im Bild verläuft eine Korngrenze und im Abstand von $\sim 20 \mu \mathrm{m}$ zur Korngrenze verläuft ein Riss (Umriss gestrichelt nachgezeichnet). Der Pfeil kennzeichnet einen Linienscan, der in Abbildung 5.21 gezeigt ist.

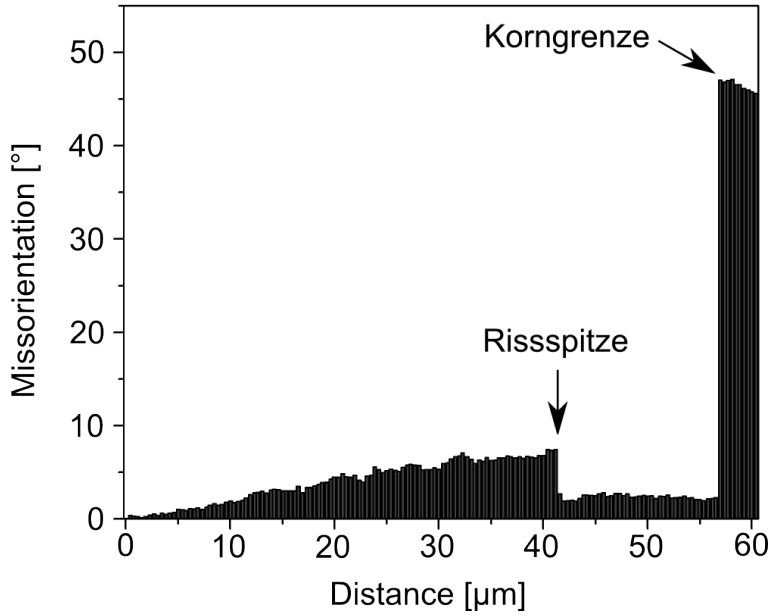

Abbildung 5.21: Bild erstellt in Zusammenarbeit mit May L. Martin, veröffentlicht in $\left[\mathrm{TML}^{+}\right.$16]. Linienscan im Bezug zu Abbildung 5.20. Es ist die Orientierung im Vergleich zum Pfeilanfang dargestellt. An der Rissspitze ist eine Rotation des Korns von circa $5^{\circ} \mathrm{zu}$ erkennen.

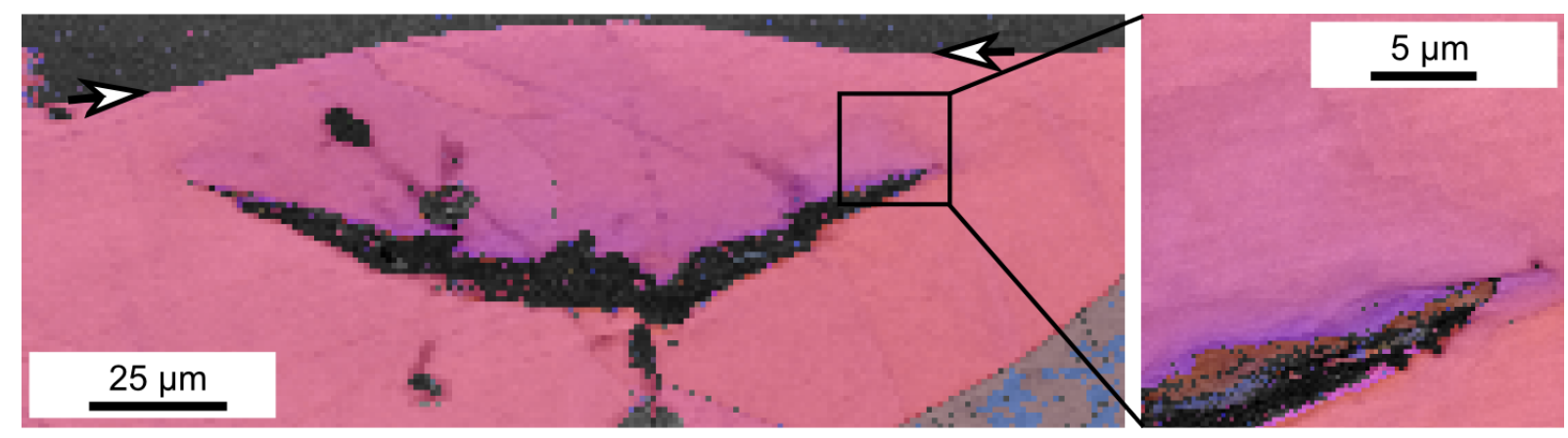

Abbildung 5.22: Bild erstellt in Zusammenarbeit mit May L. Martin. EBSD Euler Karte des Querschliffs eines Blister. Die Probenoberfläche ist mit Pfeilen markiert. Man beobachtet Kornrotation oberhalb des Risses. Die Rissspitze ist rechts in Vergrößerung dargestellt.

Anstieg in der Missorientierung zu verzeichnen, der bis zum Riss bis auf circa $7^{\circ}$ ansteigt. An der Rissspitze ist ein Sprung von etwa $5^{\circ} \mathrm{zu}$ sehen, danach ist die Rotation auf einem ähnlichen Level, wie am Beginn der Messung und vor allem ist keine Änderung in der Rotation zu beobachten. Das bedeutet in Richtung der Korngrenze ist das Material nur 
in direkter Nähe zum Riss verformt und ins Korninnere ist zusätzlich eine weitreichende Verformung von circa $30 \mu \mathrm{m}$ zu beobachten. Dieses kann aus dem Anstieg der Kornrotation auf einer Länge von etwa $30 \mu \mathrm{m}$ geschlossen werden. In Richtung der Korngrenze ist die Rotation so gut wie nicht vorhanden, da Spannungen an der Korngrenze abgebaut werden können. Eine präferierte Lage der Risse parallel zu Korngrenzen ist damit zu vermuten. In Abbildung 5.22 ist eine EBSD Messung an dem Querschliff eines Blisters durchgeführt worden. Oberhalb der Probenoberfläche, die mit Pfeilen gekennzeichnet ist, wird kein Signal gemessen. Man kann eine deutliche Rotation oberhalb des Risses erkennen. Da, im Gegensatz zu Rissen im Inneren des Materials, das Volumen des Risses durch aufschieben von Material zur Oberfläche erzeugt werden kann, ist das Material unterhalb des Risses nicht deformiert. Die Vergrößerung in Abbildung 5.22 zeigt, dass die starke Deformation des Materials nah am Riss eine Ausdehnung von circa $5 \mu \mathrm{m}$ hat, wie es auch in dem Linienscan, Abbildung 5.21, beobachtet wurde. Eine leichte Kornrotation ist bis zur Probenoberfläche zu sehen. Die Deformation, die mittels EBSD sichtbar gemacht werden konnte, ist ein weiterer Hinweis auf duktile Verformung.

\section{Bestimmung der kristallographischen Orientierung der Rissebenen}

Die kristallographischen Ebenen, entlang derer sich die Risse öffnen, werden als Rissebenen bezeichnet. Sie wurden ebenfalls mit Hilfe von EBSD-Messungen an Querschliffen der mit Wasserstoff beladenen Proben bestimmt. Wie in Abschnitt 3.2 beschrieben wurde, gleicht man die Rissebenen, siehe bunte Linien in Abbildung 5.23, mit speziellen kristallographischen Ebenen ab. Dazu wurden die Eulerwinkel des umgebenden Korns ausgelesen. Es werden jeweils die Winkel zwischen der eingezeichneten Rissebene und der kristallographischen Ebene verglichen. Die Ergebnisse für drei Risse, die sich jeweils wiederum aus Rissarmen entlang verschiedener Richtungen zusammensetzen, sind in Abbildung 5.23 aufgetragen. Eine Missorientierung von bis zu $10^{\circ}$ ist dabei zu erwarten, da die Risse einen Öffnungswinkel von bis zu $20^{\circ}$ aufweisen und die Rissebene damit nicht eindeutig ist. In der Auftragung in Abbildung 5.23 kann man erkennen, dass für alle Risse die \{100\}-Ebene als Rissebene ausgeschlossen werden kann, da der Winkel zu dieser immer größer als $20^{\circ}$ ist. Dies ist, wie in Abschnitt 2.2.2 erläutert, die Spaltebene von Eisen und eine Öffnung entlang dieser Ebene wäre bei einem reinem Spaltbruch zu erwarten. Gleitebenen $\{110\}$, $\{112\}$ oder $\{123\}$, sind als Rissebene sehr wahrscheinlich, siehe Abbildung 5.23. Betrachtet man beispielsweise den grün gekennzeichneten Arm von Riss $1(+)$, weist dieser einen Winkel von $5,5^{\circ}$ zur $\{110\}$-Ebene und jeweils Winkel über $14^{\circ}$ zu den anderen Ebenen auf. Hier kann die Rissebene entlang der $\{110\}$-Ebene als höchstwahrscheinlich eingestuft werden. Es ist nicht für jeden Rissarm der drei Risse eindeutig zu bestimmen, aber es 


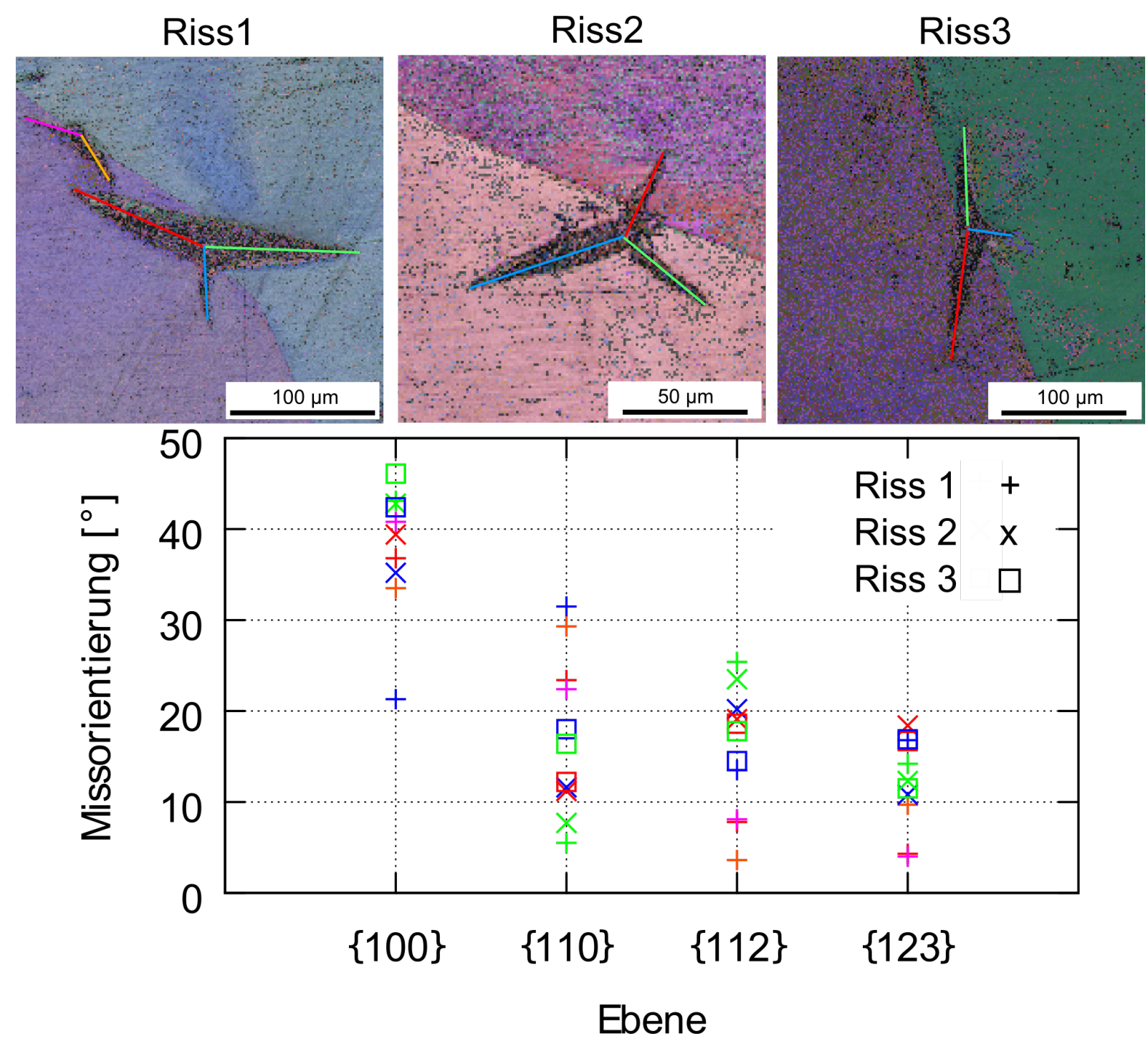

Abbildung 5.23: Zusammenarbeit mit May L. Martin. Auswertung der Rissebenen in EBSD Euler Karten. Die Rissebenen wurden mit farbigen Linien in die EBSD Messungen eingezeichnet und die Winkel zu $\{100\},\{110\},\{112\}$ und $\{123\}$ Ebenen sind in der Auftragung dargestellt. Die Farbe der Rissebene ist in der Auftragung wiederzufinden.

kommt fast immer mindestens eine der drei Gleitebenen als Rissebene in Frage. Wohingegen die Spaltebene immer ausgeschlossen werden kann. Zusammenfassend kann also festgestellt werden, dass die Risse entlang Gleitebenen verlaufen.

\section{Blisterwachstum während der Wasserstoffbeladung $\mid 5.1 .5$}

Auf den Bruchflächen der Wasserstoff-induzierten Risse wurden Stufen nachgewiesen, die als ein Halt während des Rissfortschritts interpretiert wurden, vergleiche Abbildung 5.17. Zur Bestätigung wird nun überprüft, ob ein Blister auf der Probenoberfläche während 
der Wasserstoffbeladung langsam anwächst oder schlagartig entsteht. Dafür wurden Serienaufnahmen mit einer Mikroskopkamera angefertigt. In Abschnitt 3.9 sind hierzu die experimentellen Details zu finden.

Abbildung 5.24 ist zu entnehmen, dass der dargestellte Blister mit der Zeit wächst. Man

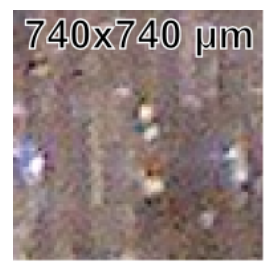

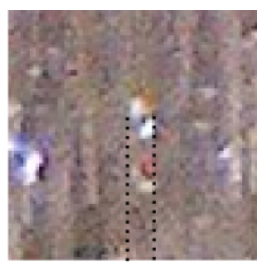

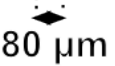

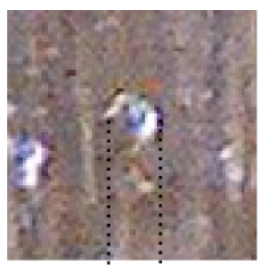

$17 \stackrel{\leftrightarrow}{\mu m}$
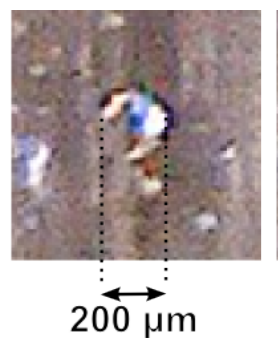

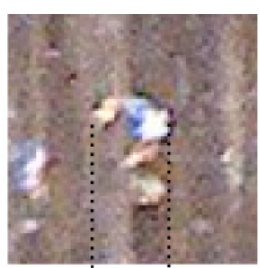

$2 \stackrel{\leftrightarrow}{\longleftrightarrow}$

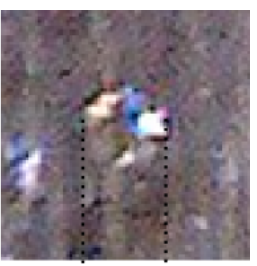

$2 \stackrel{250 \mathrm{~m}}{\longrightarrow}$

Abbildung 5.24: Serienaufnahme während der Wasserstoffbeladung erstellt mit einer Mikroskopkamera. Bilder ungefähr im Abstand von einer Minute aufgenommen.

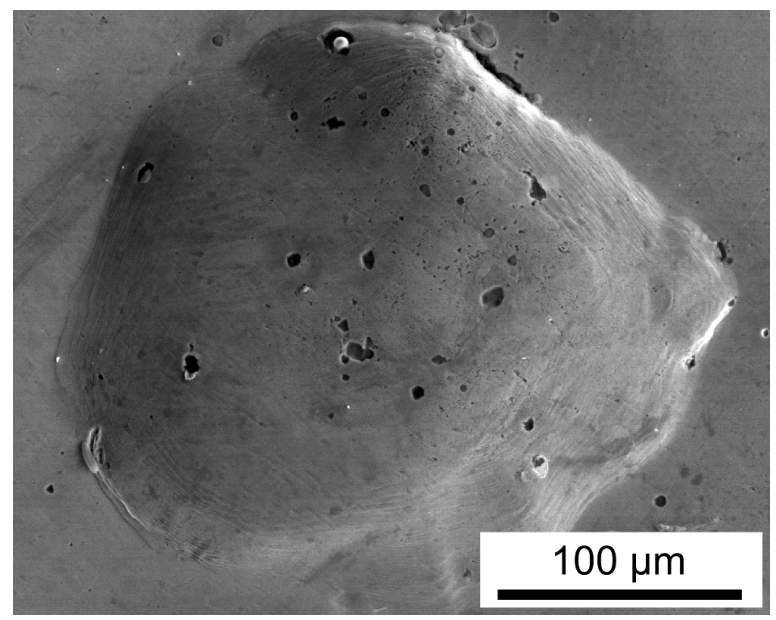

Abbildung 5.25: REM-Aufnahme des Blisters, dessen Wachstum während der Wasserstoffbeladung in Abbildung 5.24 zu sehen ist.

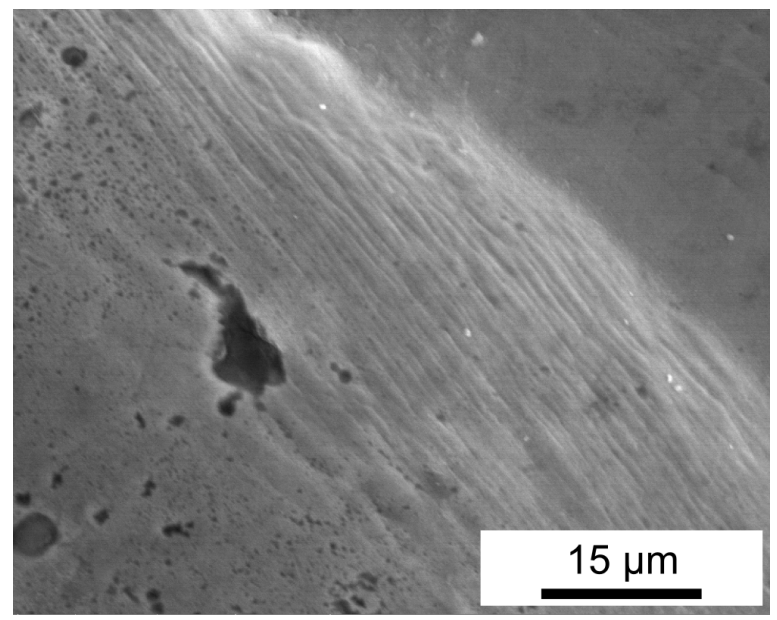

Abbildung 5.26: Vergrößerte REMAufnahme der Stufen des Blisters, der in den Abbildungen 5.24 und $5.25 \mathrm{zu}$ sehen ist.

erkennt, dass sich der in Bild 3 sichtbare Kreis in den folgenden Bildern wiederfindet und dass darum ein weißer Ring wächst. Dies kann als das Plateau und einem Hinzukommen von immer mehr Stufen um das Plateau herum identifiziert werden.

Eine REM-Aufnahme des Blisters, der in Abbildung 5.24 während des Wachstum beobachtet wurde, ist in Abbildung 5.25 und eine Vergrößerung der Stufen in Abbildung 5.26 zu sehen. Die Flanke des Blisters besteht aus etwa 30 Stufen und die Blisterentstehung wird aus dem Video auf etwa 6,7 Minuten abgeschätzt. Damit ergibt sich rein rechnerisch eine durchschnittliche Zeit von 13 s pro Stufe. Die Stufen wurden als Haltepunkte während eines diskontinuierlichen Wachstums interpretiert, somit findet circa alle $13 \mathrm{~s}$ ein Rissfortschritt statt. 


\section{\begin{tabular}{l|l} 
TEM-Untersuchung der Defektstruktur & 5.1 .6
\end{tabular}}

Zur Untersuchung der Defektstruktur des Materials, das Risse und Blister umgibt, wurden an ausgewählten Positionen TEM-Lamellen präpariert. Das Vorgehen ist in Abschnitt 3.3 beschrieben. Eine Übersicht, aus welchen Stellen die Lamellen präpariert wurden, ist in Abbildung $5.27 \mathrm{zu}$ sehen. Es wurden verschiedene TEM-Lamellen aus den Proben, deren Oberfläche mit Blistern übersät ist, geschnitten. Eine Lamelle wurde aus der Probenoberfläche zwischen den Blistern extrahiert, diese zeigt eine mögliche Defektbildung im blisterfreien Material, siehe Nummer 1 in Abbildung 5.27. Ein Querschnitt der Stufen eines Blisters ist in Nummer 2 zu sehen. Aus dem Querschliff einer beladenen Probe konnten Lamellen parallel zu Rissen extrahiert werden, siehe Nummer 3. Darüber hinaus wurden einige Lamellen aus der Bruchfäche, die beim Zugversuch der beladenen Proben entsteht, geschnitten. So wurden Querschittslamellen, die das Material unter einer Rissinitiierungsstelle zeigen, angefertigt, siehe Nummer 4. Zusätzlich konnte das Material direkt unter der Rissfläche senkrecht und parallel zur Rissausbreitung untersucht werden, siehe Nummer 5 und 6 .

Zur Vorcharakterisierung ist in Abbildung 5.28 eine TEM-Aufnahme des Ausgangsmaterials gezeigt. Diese TEM-Lamelle wurde aus dem ausgelagerten reinen Eisen (5 Stunden bei $880^{\circ} \mathrm{C}$ ) vor der Wasserstoffbeladung extrahiert. Man beobachtet in der TEM-Aufnahme, siehe Abbildung 5.28, einzelne Versetzungen, beispielsweise rechts in der Lamelle und Versetzungen, die sich aufstauen von Mitte oben nach Mitte links im Bild. Sonst ist das

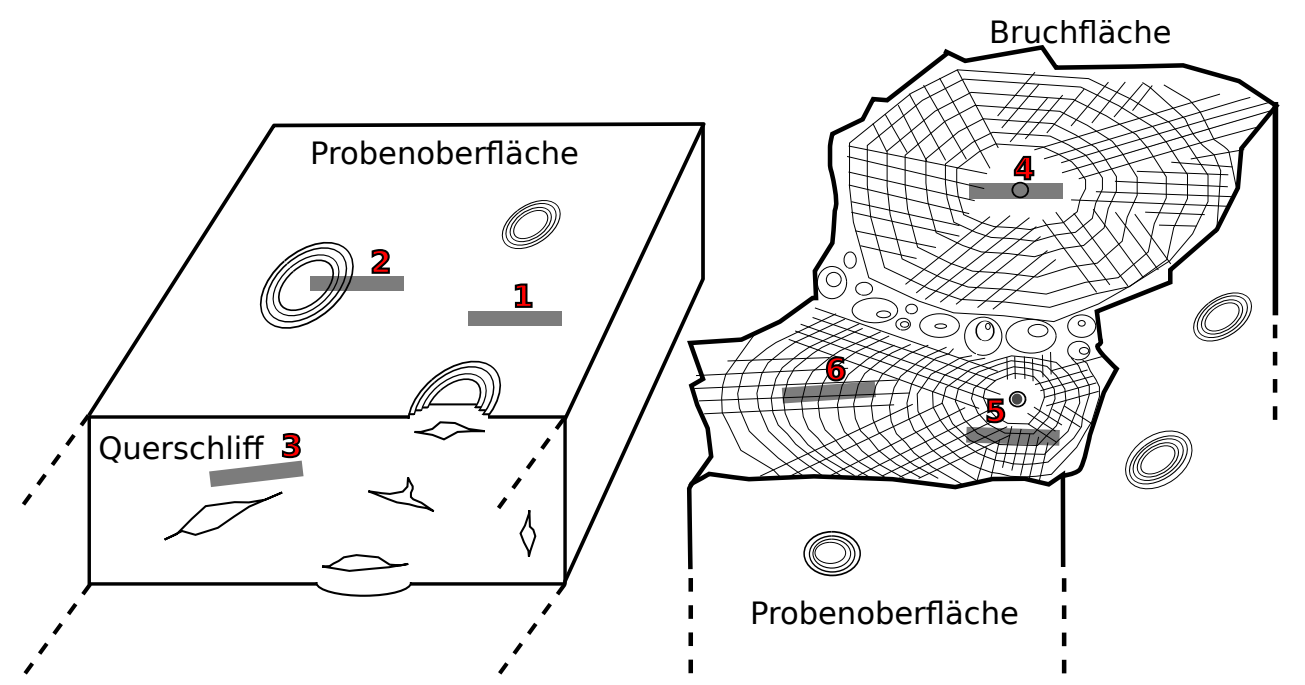

Abbildung 5.27: Schematische Darstellung, an welchen Stellen TEM-Lamellen extrahiert wurden, veröffentlicht in $\left[\mathrm{TML}^{+} 16\right]$. Graue Rechtecke entsprechen den Positionen der PlatinDeposition, hierzu senkrecht ist die spätere Lamelle angefertigt, vergleiche Abschnitt 3.3. 1: zwischen Blistern, 2: Blisterquerschnitt, 3: Parallel zu Riss, 4: Initiierungsstelle 5: senkrecht zu Rissfortschritt, 6: parallel zu Rissfortschritt. 


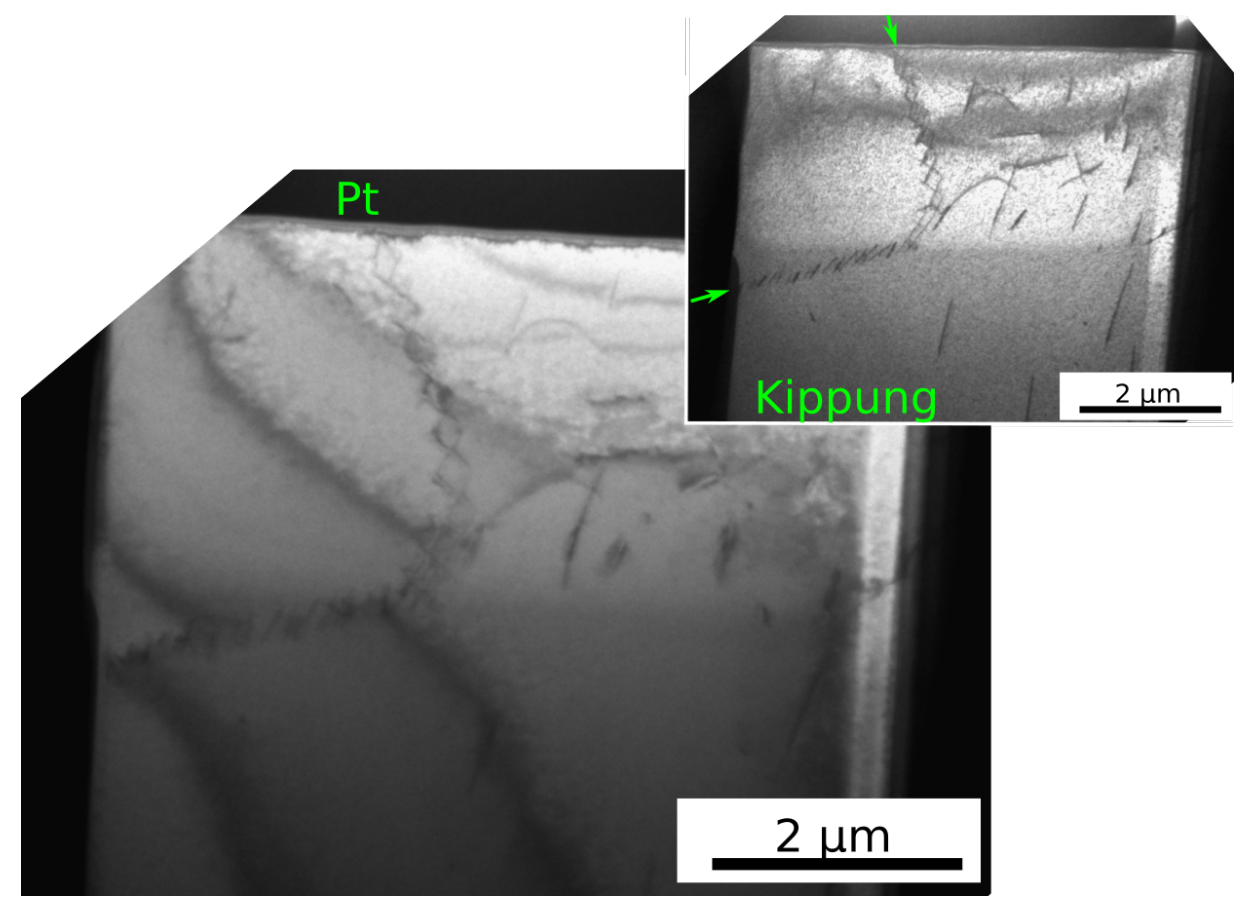

Abbildung 5.28: TEM-Aufnahme des Ausgangsmaterials Eisen nach der Auslagerung bei $880^{\circ} \mathrm{C}$ für 5 Stunden. Man erkennt Biegekonturen und einzelne Versetzungen, die sich in einem Bogen von Mitte links nach Mitte oben der Lamelle aufstauen, diese sind mit Pfeilen markiert. Oben rechts ist die Lamelle unter einer Verkippung zu sehen.

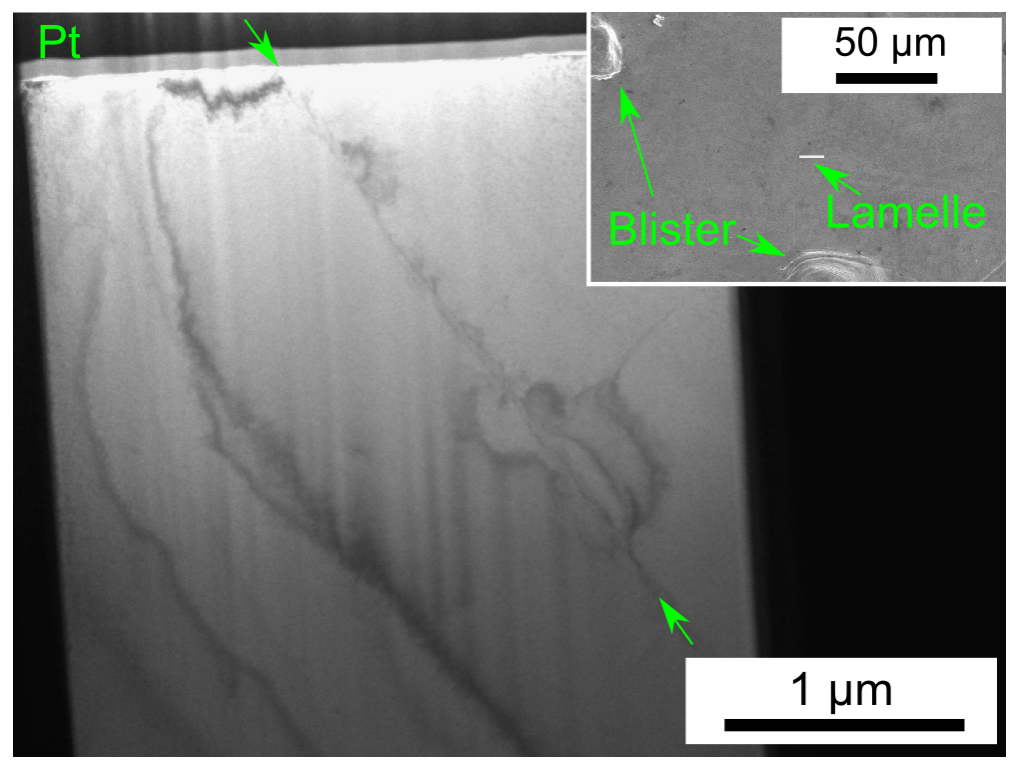

Abbildung 5.29: TEM-Aufnahme, die zwischen zwei Blistern präpariert wurde. Sie zeigt Biegekonturen und eine Kleinwinkelkorngrenze, markiert mit Pfeilen (kein Unterschied der Orientierung rechts und links der Korngrenze erkennbar). Im Vergleich zu Abbildung 5.28, kann geschlossen werden, dass keine Defekte zwischen den Blistern durch die Wasserstoffbeladung eingebracht werden. 
Material defektfrei, was vor allem während einer Kippung der Probe gut zu sehen ist, die oben rechts als zusätzliches Bild eingefügt ist. Der diffuse Hintergrund, der vor allem unter der Kippung zu beobachten ist, ist auf eine Schädigung der Oberfläche während der FIB Präparation zurückzuführen.

\section{Probe 1: Blisterfreies Material}

Abbildung 5.29 zeigt eine TEM-Lamelle, die, wie im zugehörigen REM-Bild zu sehen ist, aus einer beladenen Probe zwischen den Blistern extrahiert wurde. Aus dieser soll geschlossen werden, ob Defekte auch weit weg von den Blistern durch die Beladung oder durch die weit entfernten Blister induziert werden. Die Blister sind in diesem Fall circa $50 \mu \mathrm{m}$ entfernt. Man beobachtet nahezu defektfreies Material. Quer über die Lamelle verläuft eine Kleinwinkelkorngrenze, diese ist im TEM-Bild mit Pfeilen markiert. Das es sich um eine Kleinwinkelkorngrenze handelt, geht aus Beugungsbildern hervor, die rechts und links der Korngrenze keinen deutlichen Unterschied zeigen. Sonst sind ausschließlich Biegekonturen zu sehen, die unverformtes Material zeigen. Die Unterschiede in den Lamellen des Ausgangsmaterials und des Materials zwischen den Blistern sind höchstwahrscheinlich auf die lokalen Unterschiede in der Probe zurückzuführen. Eine weitere Erklärung könnte Wasserstoff-induzierte Herauslaufen von Versetzungen aus dem Material sein. Generell ist keine weitreichende plastische Verformung im blisterfreien Material zu beobachten.

\section{Probe 2: Querschnitt der Flanke eines Blisters}

Eine Lamelle, die aus dem Querschnitt der Flanke eines Blisters präpariert wurde, zeigt die Defektstruktur, die zu den charakteristischen Stufen des Blisters führt. In Abbildung 5.30 ist ein REM-Bild während des Präparationsprozesses im FIB gezeigt, aus der die Position der späteren Lamelle hervorgeht. Das Platin ist auf den Stufen des Blisters deponiert. Eine weitere REM-Aufnahme, Abbildung 5.31, zeigt, dass die spätere Lamelle auf dem Riss endet, der mit dem Blister in Zusammenhang steht. Das bedeutet, dass die gesamte Mikrostruktur zwischen Riss und Probenoberfläche in dieser Lamelle untersucht werden kann. Außerdem ist in Abbildung 5.31 zu erkennen, dass Material den Riss umgibt, das im REM einen veränderten Kontrast erzeugt. Die Abgrenzung ist durch eine dunkle Linie zu erkennen, die im REM-Bild mit Pfeilen gekennzeichnet ist. Die Schicht ist oberhalb des Risses etwa $3 \mu \mathrm{m}$ und unterhalb etwa $1 \mu \mathrm{m}$ dick. Sie ist im TEM-Bild, Abbildung 5.32, als nicht durchstrahlbares schwarzes Material wiederzufinden. Weiter wird beobachtet, dass der Riss zum Ende hin an einer Stelle deutlich schmaler wird. Die TEM-Aufnahme der Lamelle ist in Abbildung 5.32 abgebildet. Oben im Bild sieht man die Schutzschicht aus Platin, das auf der Flanke des Blisters deponiert wurde. Man erkennt an der Grenzfläche zwischen Eisen und Platin die Stufen. Am unteren Rand des Bildes ist undurchstrahlbares 


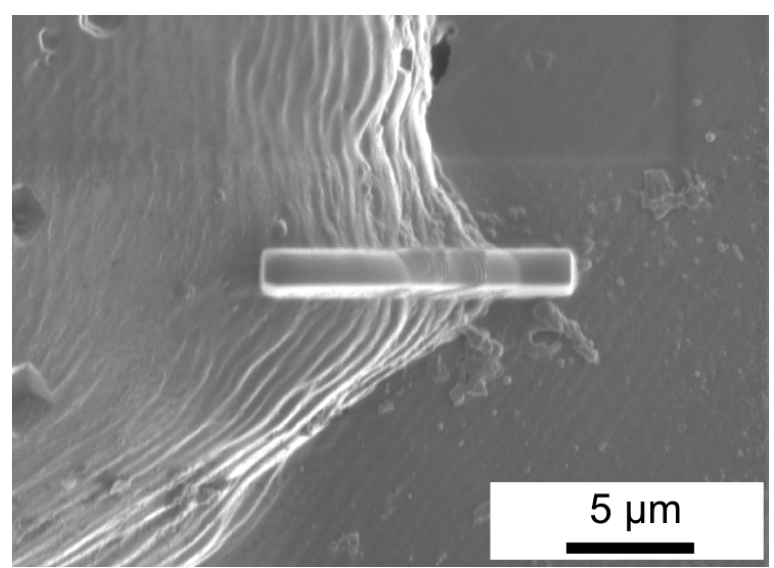

Abbildung 5.30: REM-Aufnahme während des FIB Prozesses. Unterhalb der Platin Deposition ist die spätere Lamelle präpariert worden.

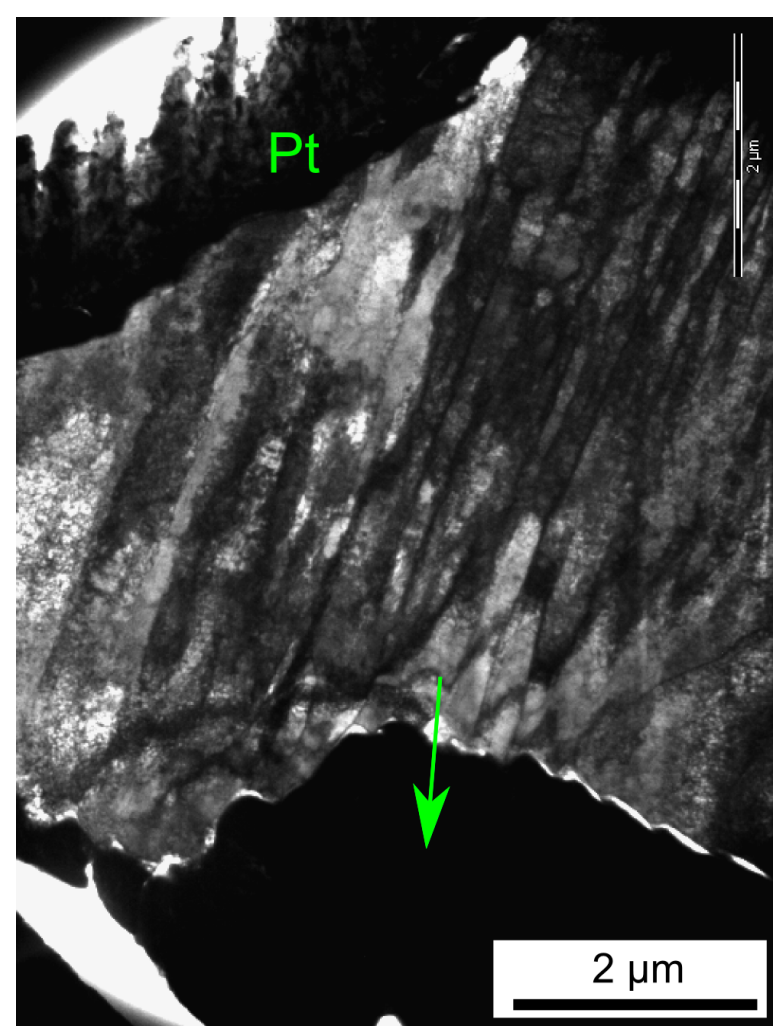

Abbildung 5.32: Eigene Ergebnisse veröffentlicht in $\left[\mathrm{TML}^{+} 16\right]$. TEM-Aufnahme, die die Mikrostruktur des Blisterquerschnitts zeigt. Um den Riss ist undurchstrahlbares Material, das durch einen EDX-Linienscan entlang des Pfeiles in Abb. 5.34 analysiert wird.

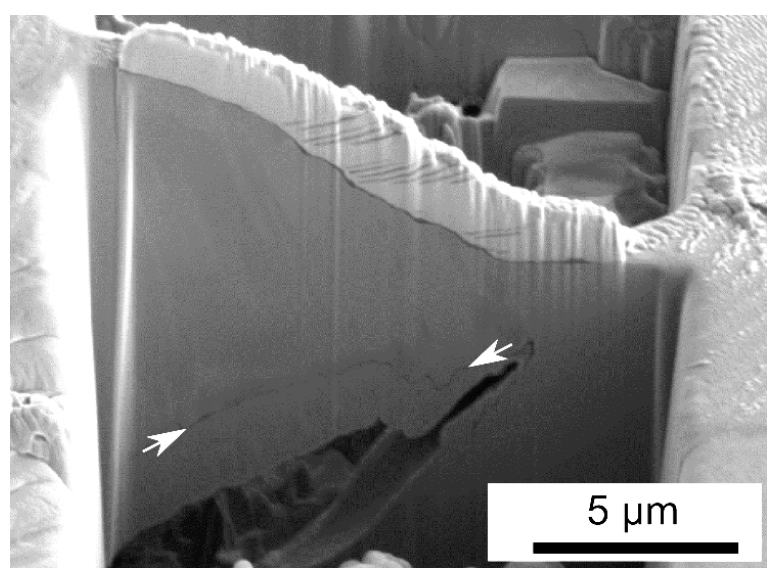

Abbildung 5.31: REM-Aufnahme während des FIB Prozesses. Lamelle endet auf einem Riss. Die Pfeile kennzeichnen eine Materialveränderung um den Riss.

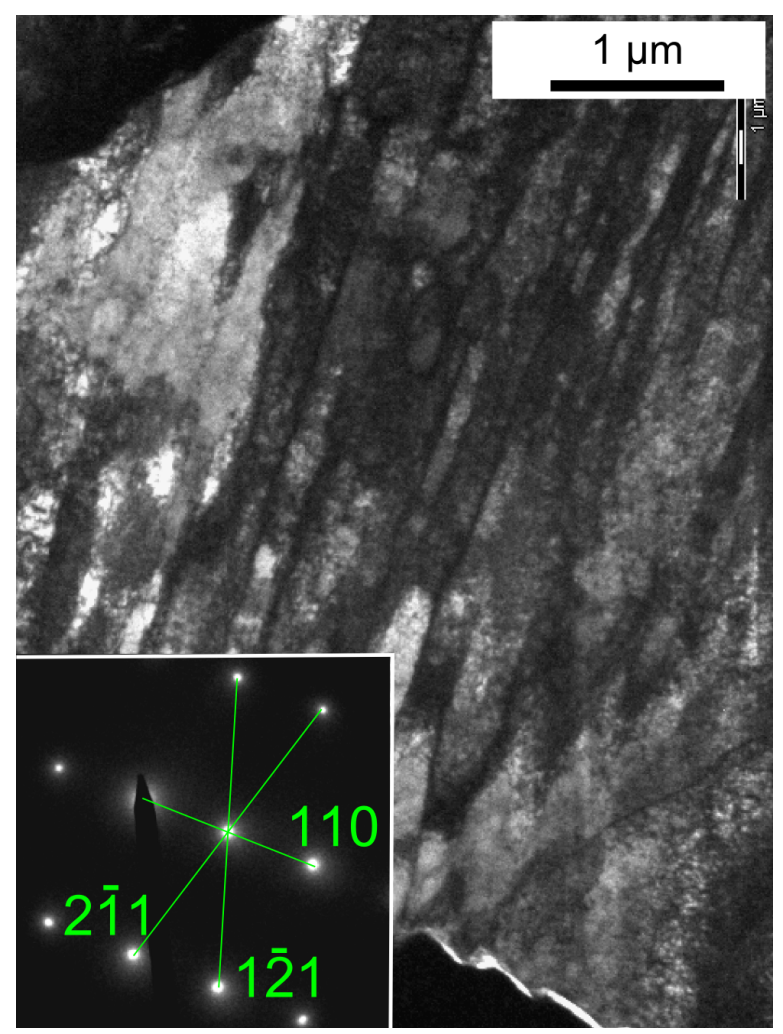

Abbildung 5.33: Vergrößerter Ausschnitt der TEM-Aufnahme mit Analyse der kristallographischen Richtungen. Das unten links gezeigte Beugungsbild ist rotationskalibriert. 


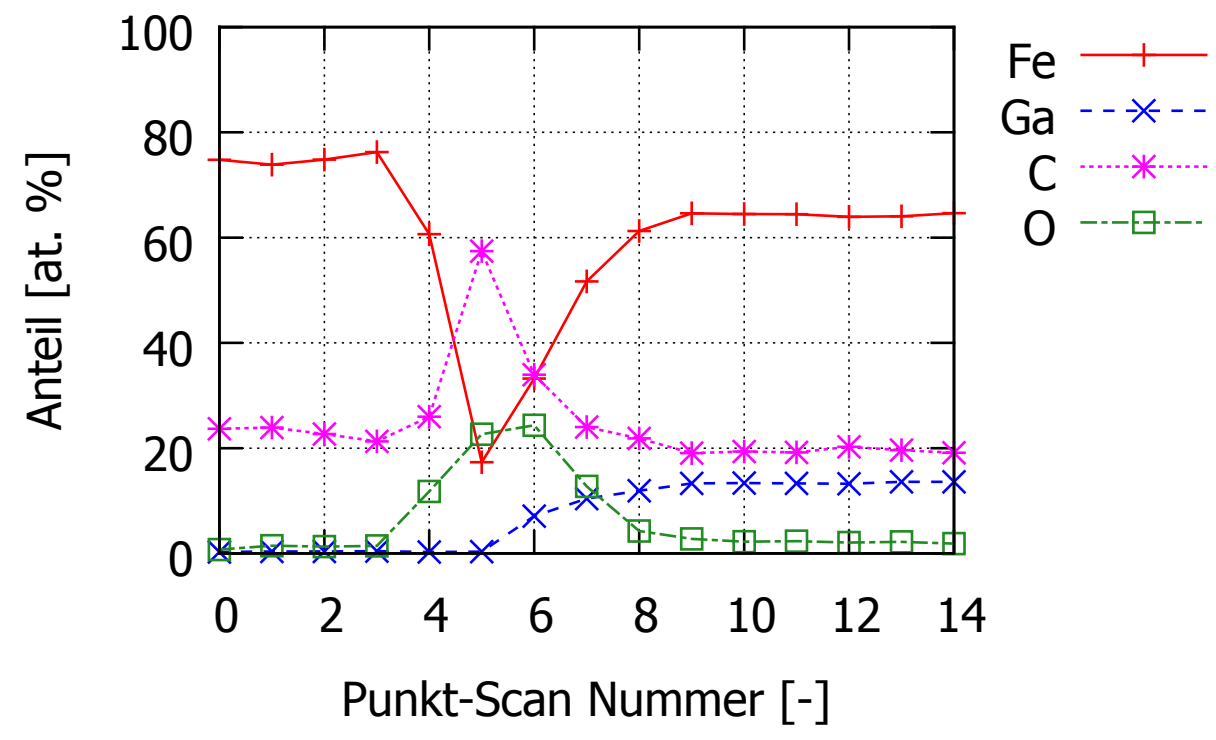

Abbildung 5.34: EDX-Scans wurden in Zusammenarbeit mit Martin Deutges durchgeführt. EDX-Linienscan entlang des Pfeils, der im TEM-Bild, Abbildung 5.32, zu sehen ist. Die ersten drei Punkte zeigen reines Eisen. Der Kohlenstoff ist eine Verunreinigung der Oberfläche. Danach steigt der Gallium-Anteil erheblich. Es handelt sich somit um Redeposition eines Eisen-Gallium Gemischs in den Riss.

Material zu sehen, dass schon in den REM-Bildern durch einen veränderten Kontrast zu erahnen war. Eine Analyse dessen ist durch einen EDX-Linienscan erfolgt, der durch den Pfeil im Bild gekennzeichnet ist. Die resultierende EDX-Messung ist in Abbildung 5.34 dargestellt. Die ersten vier Messpunkte sind im Material des Blisters und man misst Eisen und, wie bei einer EDX-Messung im TEM üblich eine Kohlenstoffkontamination, die höchstwahrscheinlich auf der Oberfläche der Lamelle ist. Messpunkt Nummer 5 befindet sich in einem Loch zwischen Eisen und dem unbekannten Material. Man misst hier hauptsächlich Kohlenstoff und Sauerstoff, was wiederum auf die Kontamination der Oberfläche der Lamelle zurückzuführen ist. In dem undurchstrahlbaren Material wird neben Eisen eine erhebliche Menge an Gallium gemessen. Dies ist während der FIB Präparation in das Material gelangt. Da der Gehalt an Gallium im Rest der Lamelle nahezu Null ist, kann darauf geschlossen werden, dass es sich bei dem schwarzen Material um redeponiertes Material aus Eisen der Probe und Gallium aus dem Ionenstrahl handelt. Der schwarze Bereich stellt somit den ursprünglichen Riss dar.

An der Grenzfläche vom Eisen zum Riss sind Stufen erkennbar, diese wurden auch im REM auf den Rissflächen nachgewiesen, vergleiche Abbildung 5.17, und als Haltepunkte während des Rissfortschritts identifiziert. Zwischen der Rissfläche und der Probenoberfläche sind parallel angeordnete Scherbänder zu sehen. Bei genauere Betrachtung der Abstände der Scherbänder und der Stufen auf der Rissfläche, kann man darauf schließen, dass die Scherbänder Verbindungen zwischen den Stufen auf der Rissfläche und den 
Stufen des Blisters auf der Probenoberfläche darstellen. Abbildung 5.33 ist eine leicht vergrößerte Darstellung der TEM-Lamelle, in welcher eine Analyse der kristallographischen Orientierung der Rissfläche durchgeführt wurde. Unten links in der Abbildung ist das Beugungsbild zu sehen, welches nach der in Abschnitt 3.4 beschriebenen Prozedur rotationskalibiert wurde. Daher können die kristallographischen Richtungen direkt in das Realbild übertragen werden. Es können die Stufen auf der Rissfläche den $\{110\}$ Ebenen zuordnen werden, was die Rissöffnung entlang Gleitebenen aus den EBSD-Messungen bestätigt, vergleiche Abschnitt 5.1.4.

\section{Probe 3 Mikrostruktur parallel zu einem Riss}

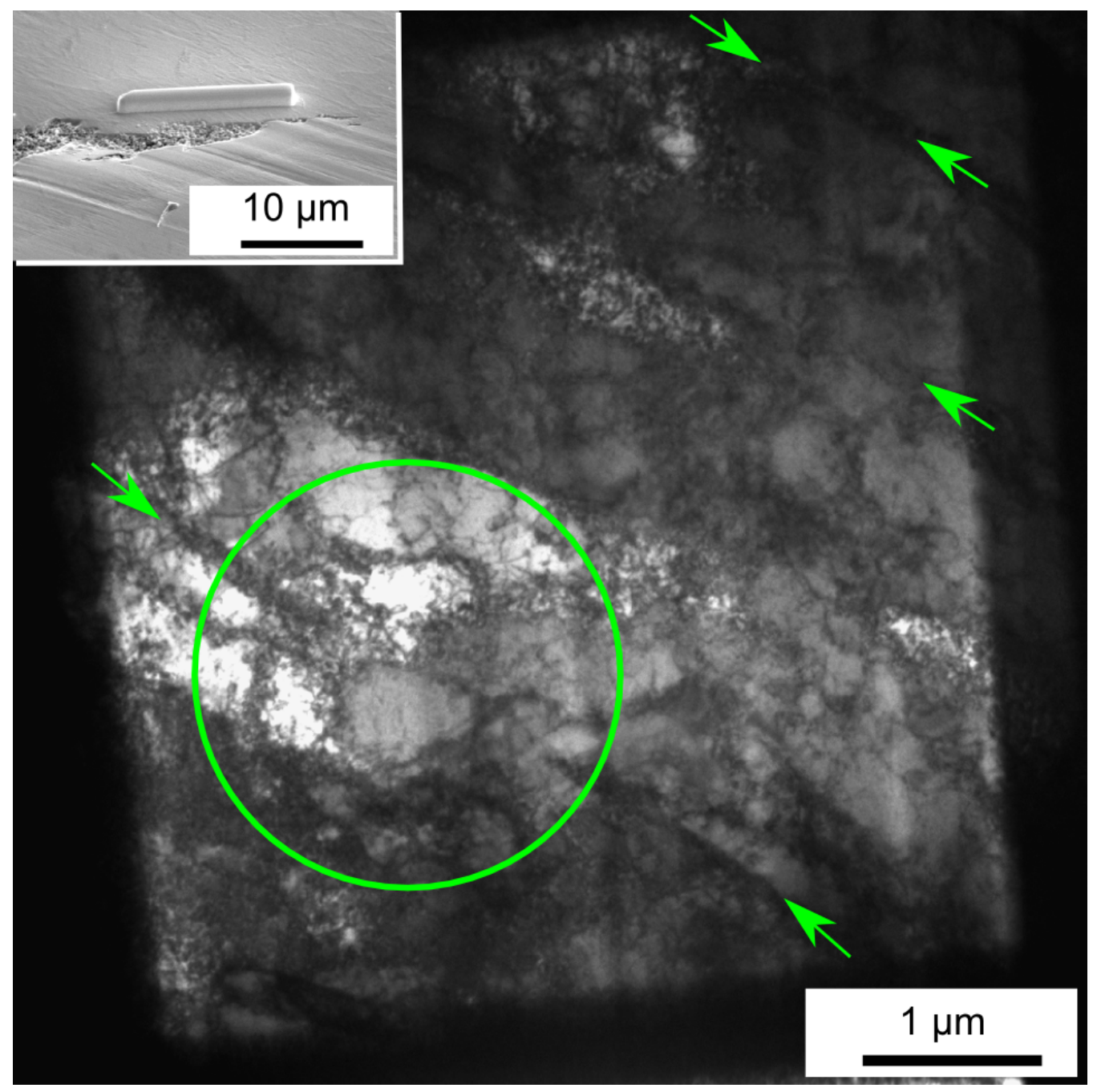

Abbildung 5.35: Eigene Ergebnisse veröffentlicht in [TML $\left.{ }^{+} 16\right]$. TEM-Aufnahme einer Lamelle parallel zu einem Riss, vergleiche REM-Bild oben links. Man erkennt parallele Scherbänder, markiert mit Pfeilen und eine Anordnung von Versetzungen in Zellwänden, markiert mit einem Kreis. 
Die Defektstruktur parallel und in einigen Mikrometern Entfernung zu einem Riss ist in Abbildung $5.35 \mathrm{zu}$ sehen. Eine REM-Abbildung oben links im Bild zeigt, wo die Lamelle präpariert wurde. In der gesamten Lamelle ist eine hohe Versetzungsdichte zu beobachten, die jedoch nicht näher quantifiziert werden kann. Es werden parallele Strukturen beobachtet, welche im TEM-Bild mit Pfeilen gekennzeichnet sind. Hierbei handelt es sich um Scherbänder, die auch in der Querschnittslamelle des Blisters, Abbildung 5.32, beobachtet wurden. In dieser TEM-Aufnahme sind zusätzlich weitere Merkmale erkennbar, die auf plastische Verformung schließen lassen. Innerhalb des Kreises in Abbildung 5.35 ist eine Anordnung von Versetzungen in Zellwänden, eine Vorstufe zur Subkornbildung, zu sehen. Eine geringfügig andere Orientierung in den Subkörnern ist an einem veränderten Kontrast zum umliegenden Material erkennbar.

\section{Probe 4: Mikrostruktur um Rissinitiierungsstelle}

Nichtmetallische Einschlüsse konnten in Abschnitt 5.1.3 als Ausgangspunkt für die Risse identifiziert werden. Die Einschlüsse wurden separiert von der Eisenmatrix in Löchern gefunden. Um die Defektstruktur direkt um die Rissinitiierungsstelle zu untersuchen, wurde eine Lamelle über ein Loch, das zu einem Riss führte, präpariert. In der REM-Aufnahme, links unten in Abbildung 5.36, ist angedeutet, wo die Lamelle extrahiert wurde. In diesem Fall ist nicht die Platin-Deposition abgebildet, da sie das Loch bedeckt und es dadurch nicht mehr sichtbar wäre. Die TEM-Aufnahme der Lamelle ist in Abbildung 5.36 zu sehen. Es wurde markiert, wo Platin in das Loch deponiert wurde. Man erkennt ausgehend von dem Loch konzentrische Biegekonturen. Dies lässt auf einen hohen Druck schließen, der innerhalb des Loches geherrscht hat, bevor der Riss initiiert wurde. Dieser Druck verformte die umliegende Matrix konzentrisch, wie es aus der TEM-Aufnahme hervorgeht, bevor der Riss initiiert wurde. Weiter ist in der Aufnahme erkennbar, dass Scherbänder vor allem im linken Teil der Lamelle erzeugt wurden. Diese wurden auch schon im Blisterquerschnitt und in der Lamelle parallel zu einem Riss nachgewiesen, vergleiche die Abbildungen 5.32 und 5.35. Sie sind damit das typische Verformungsmerkmal und hier ist zu sehen, dass sie in direkter Umgebung zur Initiierungsstelle entstehen. Man erkennt, dass die Scherbänder unterhalb des Loches enden, beziehungsweise anfangen. 


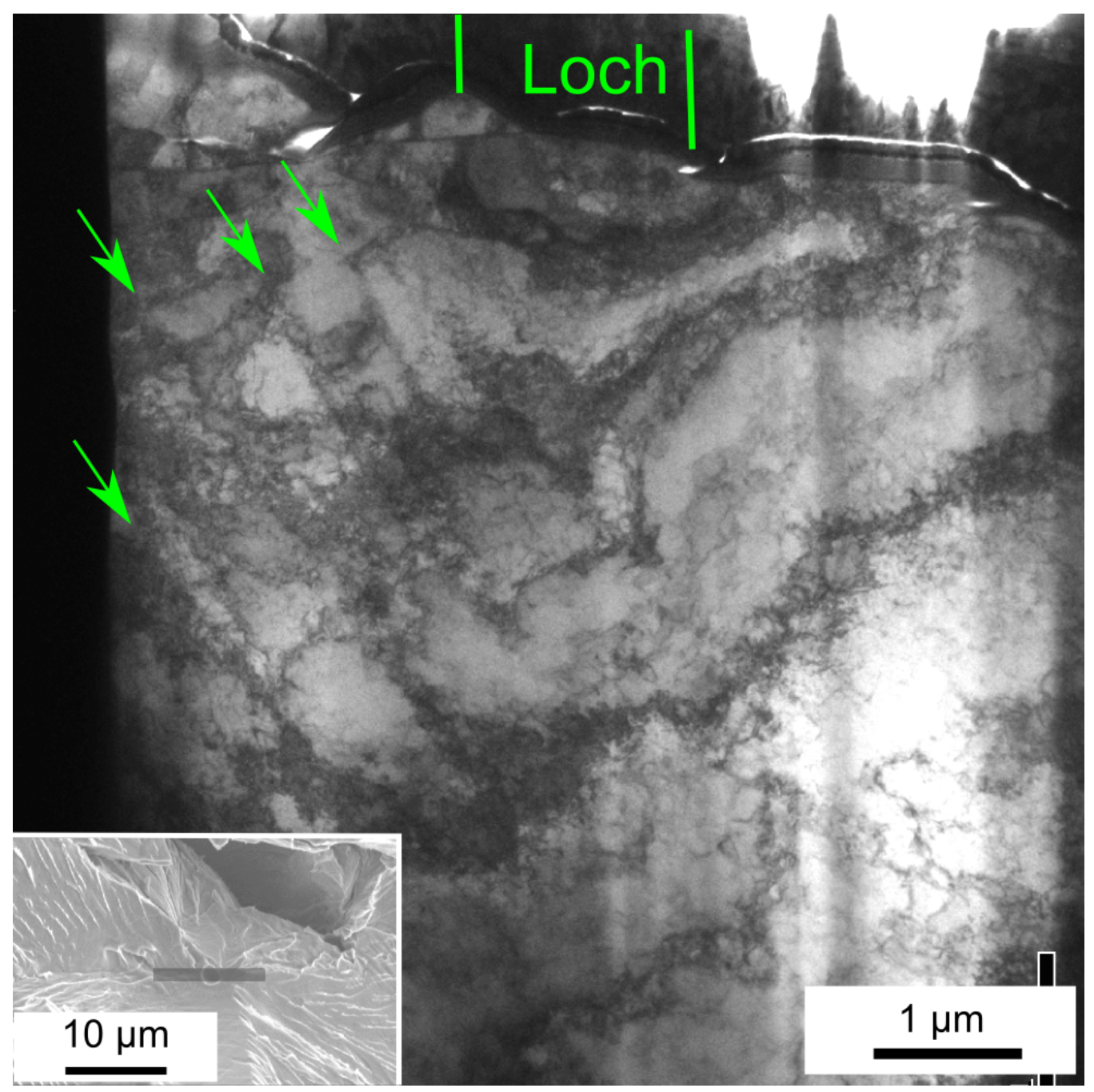

Abbildung 5.36: Eigene Ergebnisse veröffentlicht in $\left[\mathrm{TML}^{+} 16\right]$. TEM-Aufnahme der Mikrostruktur unter einem Loch, in dem ursprünglich ein Einschluss war, und von dem aus der Riss initiiert wurde. Die REM-Aufnahme unten links zeigt die Position, an der die TEM-Lamelle extrahiert wurde.

\section{Probe 5 und Probe 6: Rissfortschritt}

Es wurden auf der Rissfläche der Wasserstoff-induzierten Brüche Stufen und Streifen auf diesen Stufen nachgewiesen, vergleiche Abbildung 5.17. Um die Entstehung mikrostrukturell zu verstehen, wurden zwei Lamellen jeweils über diesen Merkmalen angefertigt. Abbildung 5.37 zeigt die Mikrostruktur, die zu den Stufen auf der Rissfläche führt. Man erkennt, wie auch in der TEM-Aufnahme des Blisterquerschnitts, Abbildung 5.10, Scherbänder. Es zeigt sich auch bei einem Riss innerhalb des Materials, der nicht zu einem Blister auf der Oberfläche führt, dass die Öffnung des Risses mit Materialverschiebung entlang von Scherbändern einhergeht.

In Abbildung 5.38 ist die TEM-Lamelle zu sehen, die senkrecht zum Rissfortschritt prä- 


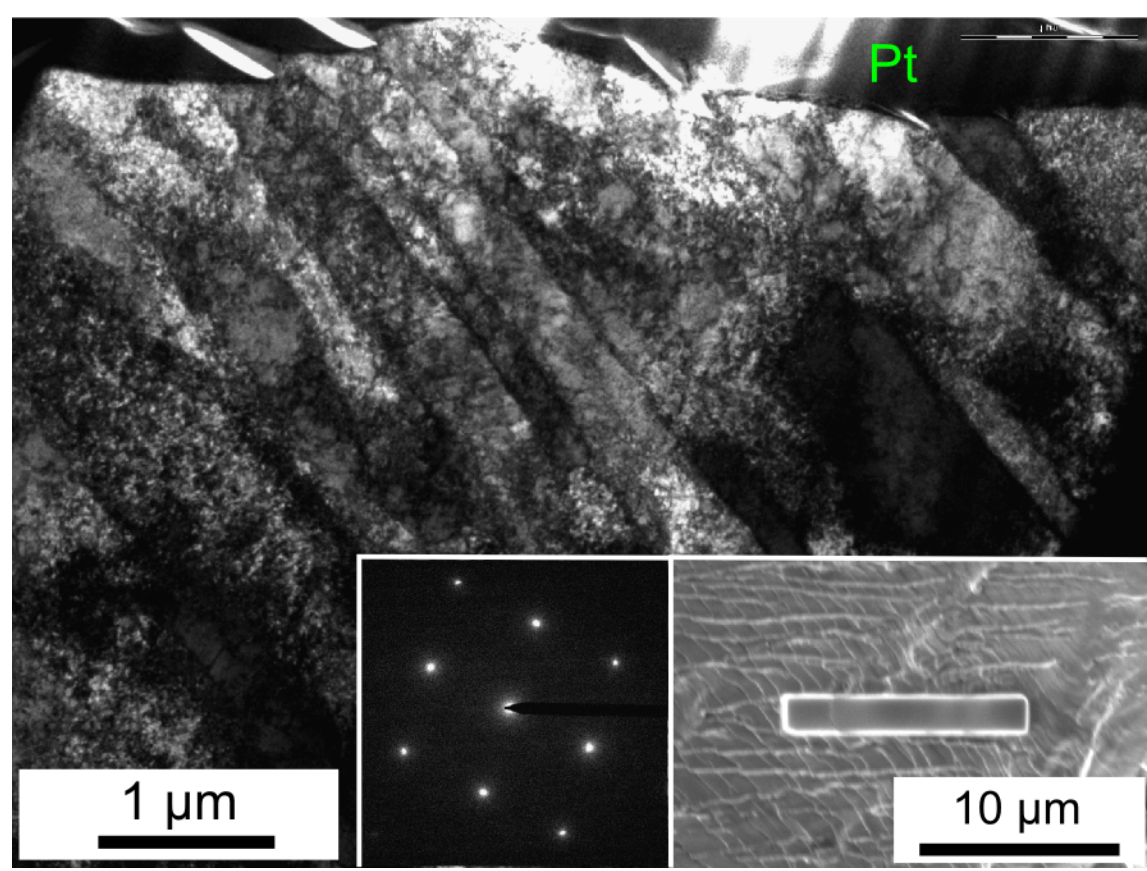

Abbildung 5.37: Eigene Ergebnisse veröffentlicht in $\left[\mathrm{TML}^{+} 16\right]$. TEM-Aufnahme, die senkrecht zu den Stufen präpariert wurde. Sie zeigt die Mikrostruktur parallel zum Rissfortschritt. Position der Lamelle entspricht Probe 6 in Abbildung 5.27.

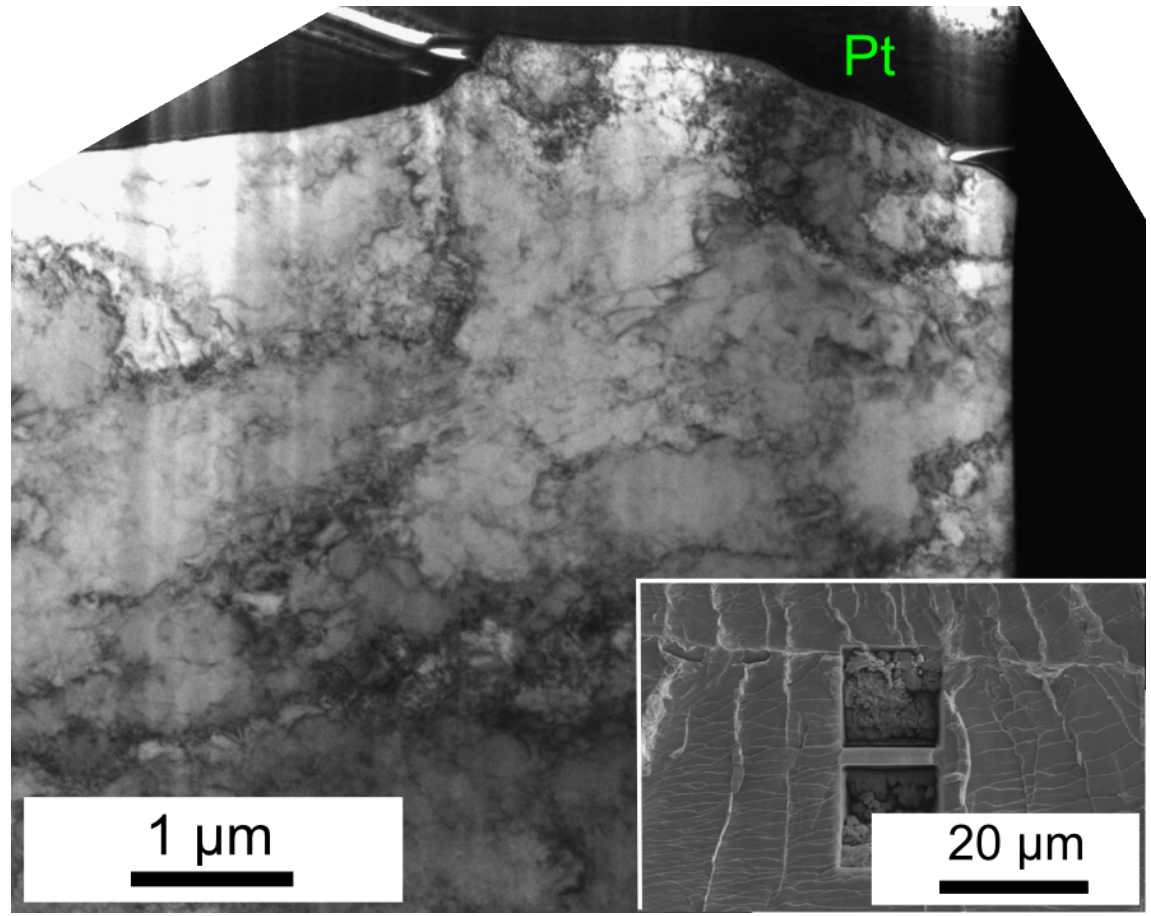

Abbildung 5.38: Eigene Ergebnisse veröffentlicht in [TML $\left.{ }^{+} 16\right]$. TEM-Aufnahme, die die Mikrostruktur der Streifen zeigt, die auf den Stufen zu finden sind. Die Lamelle wurde senkrecht zu einem solchen Merkmal präpariert, siehe REM-Aufnahme unten rechts, vgl. Probe 5 in Abb. 5.27. 
pariert wurde. Diese ist demnach senkrecht zu den Streifen präpariert, die typischerweise auf Stufen in Rissausbreitungsrichtung verlaufen. Aus der TEM-Aufnahme geht hervor, dass die Streifen auf den Stufen nicht mit speziellen Merkmalen im Material unter der Rissfläche zu korrelieren sind. Man erkennt den Streifen in der Form der Platin-Deposition wieder. In der Mikrostruktur, die in der Lamelle zu sehen ist, sind allerdings keine speziellen Merkmale von starker plastischer Verformung, wie Scherbänder, zu finden. Aber eine deutlich erhöhte Versetzungsdichte ist, vor allem im Vergleich zum Ausgangsmaterial, vergleiche Abbildung 5.28, zu sehen.

\section{\begin{tabular}{l|l} 
Einfluss durch Veränderung des Materials & 5.1 .7
\end{tabular}}

Alle gezeigten Ergebnisse bezogen sich bisher auf das reine ausgelagerte Eisen. Nun werden die Veränderungen untersucht, die in ARMCO-Eisen und reinem Eisen mit kleinen Körnern auftreten. Die verschiedenen Proben wurden in Kapitel 4 eingeführt. Zunächst wird die Verteilung der Blister auf der Oberfläche betrachtet. Dabei ist zu beachten, dass reines Eisen und ARMCO-Eisen sehr gut miteinander verglichen werden können, da es sich um eine sehr ähnliche Probengeometrie handelt und die Auslagerung identisch ist. Um Proben des reinen Eisens mit kleineren Körnern herzustellen, wurde, wie in Kapitel 4 beschrieben, das Eisen gewalzt und bei $600^{\circ} \mathrm{C}$ für 2 Stunden ausgelagert. Daher sind die Proben dünner und es wurde eine etwas niedrigere Stromdichte zur Beladung gewählt. Diese Proben werden im Folgenden immer als reines Eisen mit kleinen Körnern bezeichnet. Man beobachtet in den Abbildungen 5.39 bis 5.41 leichte Unterschiede in der Verteilung der Blister auf der Oberfläche und ebenfalls bei den Rissen im Querschnitt des Materials. Das ARMCO-Eisen weist eine deutlich höhere Bedeckung mit Blistern auf.

Die Querschnitte der Proben erklären diese Veränderung in der Verteilung, denn bei ARMCO-Eisen sind die Risse alle direkt unter der Probenoberfläche zu finden, während sie bei den beiden reinen Eisenproben über den gesamten Querschnitt verteilt sind. Dadurch wird im Falle von ARMCO-Eisen durch jeden Riss ein Blister auf der Oberfläche erzeugt, was bei Rissen, die tief im Material liegen, nicht der Fall ist. Die etwas höhere Bedeckung mit Blistern bei reinem Eisen mit kleinen Körnern im Vergleich zu reinem Eisen liegt höchstwahrscheinlich an der geringeren Probendicke.

\section{Wasserstoffgehalt der Proben $\mid 5.1 .8$}

Bei der Untersuchung der Mikrostruktur von Rissen und dessen Initiierungsstellen wurde vermutet, dass sich der Wasserstoff gasförmig in den Rissen und vorher in den Löchern um die Einschlüsse befindet. Dies wird mit einer Messung des Gesamtwasserstoffgehaltes veri- 


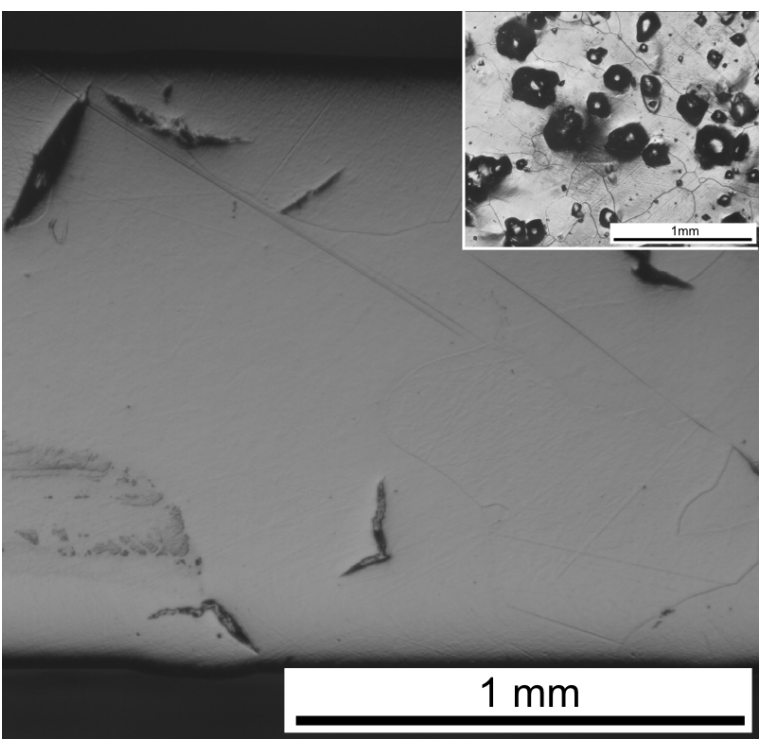

Abbildung 5.39: Optische Mikroskopaufnahme des Querschliffs und der Blister von reinem Eisen. Blister auf der Oberfläche haben relativ großen Abstand. Risse sind über den gesamten Querschnitt der Probe zu sehen.

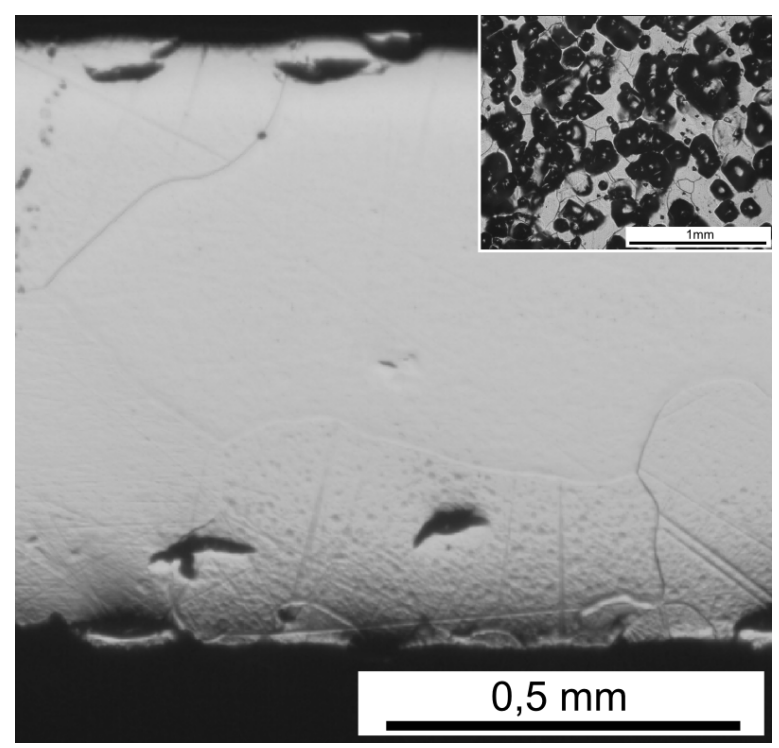

Abbildung 5.40: Optische Mikroskopaufnahme von ARMCO-Eisen. Blister bedecken die Oberfläche zu ca. 50\%. Risse sind nur direkt unter der Oberfläche zu finden. Es wurde die gleiche Beladung wie bei Reineisen verwendet.

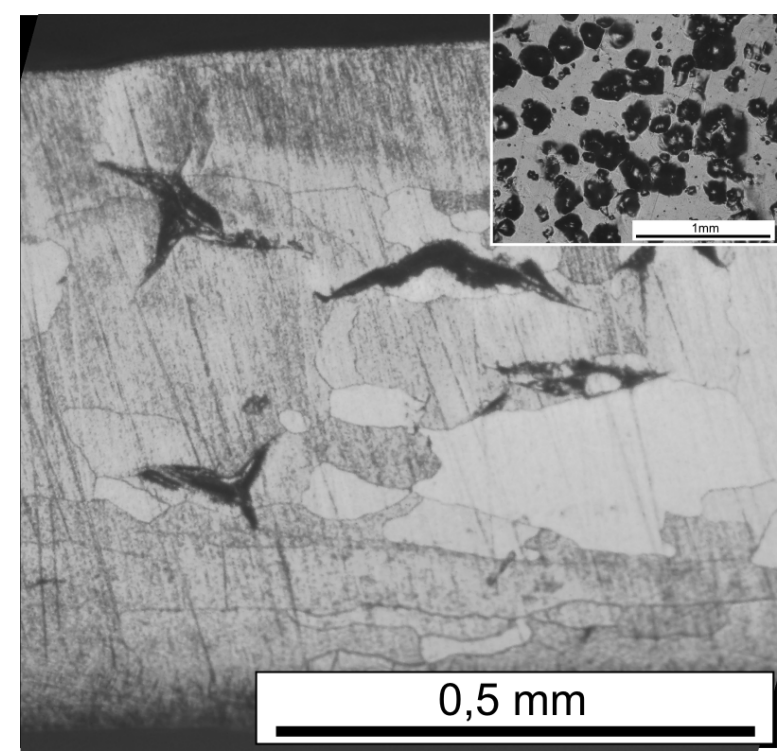

Abbildung 5.41: Optische Mikroskopaufnahme des reinen Eisen mit kleinen Körnern. Risse durchziehen den gesamten Querschnitt der Probe. 
fiziert. Dazu wird die in Abschnitt 3.5 beschriebene Schmelzextraktionsanalyse verwendet. Bei dieser Methode kann nicht zwischen im Gitter gelösten und gasförmigen Wasserstoff in Hohlräumen unterschieden werden.

Es wurde ein Reihenversuch durchgeführt, indem Eisenproben jeweils eine halbe Stunde bei verschiedenen Stromstärken beladen wurden und direkt im Anschluss wurde der Wasserstoffgehalt bestimmt. Der resultierende Wasserstoffgehalt ist als Funktion der Be-

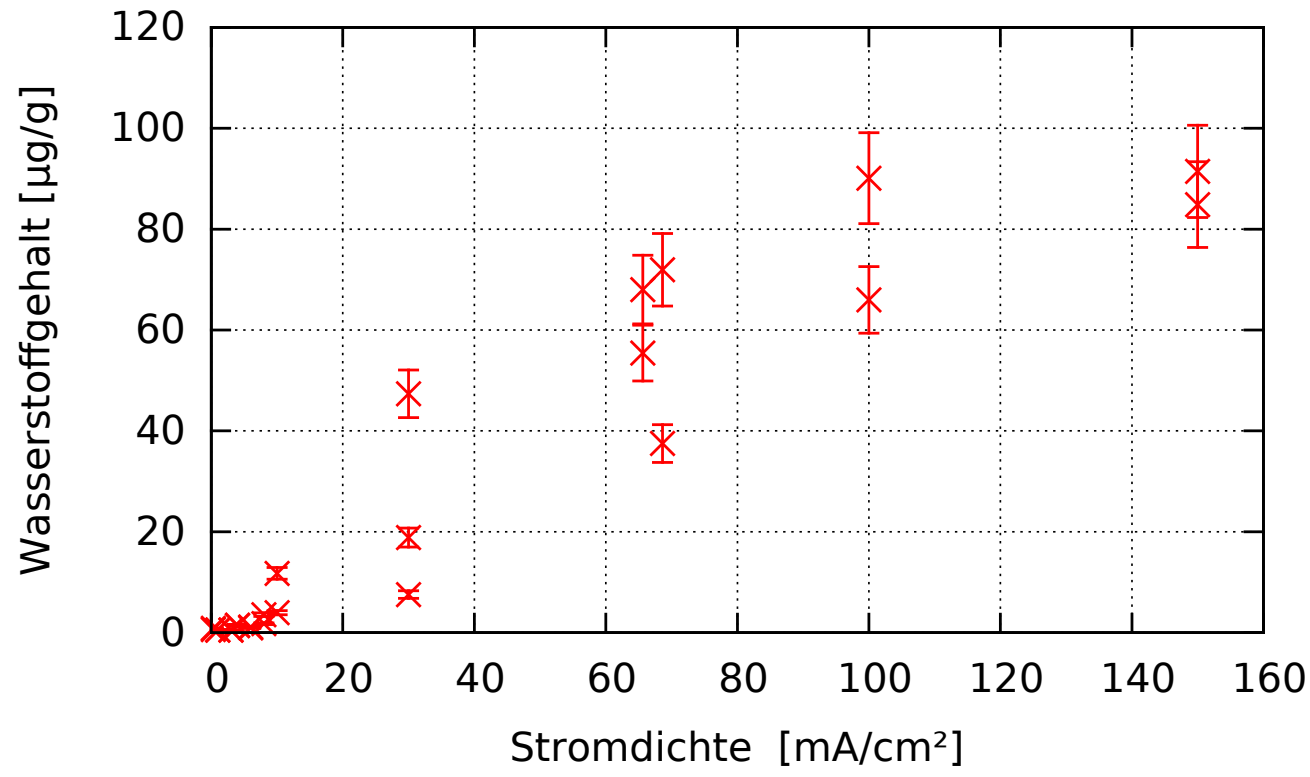

Abbildung 5.42: Eigene Ergebnisse veröffentlicht in $\left[\mathrm{TML}^{+} 16\right]$. Auftragung des Gesamtwasserstoffgehaltes in der Probe in Abhängigkeit der Beladestromstärke. Beladezeit war jeweils 30 Minuten.

ladestromdichte in Abbildung 5.42 aufgetragen. Man beobachtet, dass unterhalb einer Beladestromstärke von $8 \mathrm{~mA} / \mathrm{cm}^{2}$ etwa $1 \mu \mathrm{g} / \mathrm{g}$ Wasserstoff in der Probe vorliegt. Dies ist deutlich mehr als die theoretische Löslichkeit von Wasserstoff in Eisen bei Raumtemperatur $\left(2 \cdot 10^{-8}\right.$ at. $\%$ bei $0,1 \mathrm{MPa}$ Wasserstoffdruck). $1 \mu \mathrm{g} / \mathrm{g}$ wird allerdings häufig in Eisen als Löslichkeitsgrenze bei Raumtemperatur angegeben, wobei der größte Anteil des Wasserstoffs in diesem Fall an Defekten im Material zu finden ist [Nag16]. Das bedeutet, wenn mit einer Stromdichte kleiner als $8 \mathrm{~mA} / \mathrm{cm}^{2}$ beladen wird, entspricht der Wasserstoffgehalt der maximalen Löslichkeit von Wasserstoff in Eisen. Bei einer Beladung mit höheren Stromstärken steigt der Gehalt im Material rasch mit Erhöhen der Stromdichte an. Ebenfalls ab $8 \mathrm{~mA} / \mathrm{cm}^{2}$ werden Blister auf der Oberfläche des Materials beobachtet. Bei genau $8 \mathrm{~mA} / \mathrm{cm}^{2}$ sind einzelne Blister zu beobachten und ab etwa $100 \mathrm{~mA} / \mathrm{cm}^{2}$ ist die Oberfläche übersät mit Blistern. Ab circa $100 \mathrm{~mA} / \mathrm{cm}^{2}$ tritt eine Sättigung von knapp $100 \mu \mathrm{g} / \mathrm{g}$ (=5,3 at.\%) auf und es kann durch Erhöhung der Stromdichte nicht mehr Wasserstoff gelöst werden. Es wird also ein direkter Zusammenhang zwischen Blistern auf der Oberfläche und einem Anstieg in der Wasserstoffkonzentration in der Probe beobachtet. 
Dieser Zusammenhang ist auch in der Sättigung der Kurve und einer nicht mehr steigenden Bedeckung der Oberfläche mit Blistern wiederzufinden. Dies bestätigt die Vermutung, dass der größte Anteil an Wasserstoff als Gas in den Rissen vorliegt. Welcher Gasdruck dabei in den Rissen entsteht, wird im folgenden Abschnitt bestimmt.

\section{Messung des Wasserstoffdrucks in den Rissen $\quad 5.1 .9$}

Die Messung des Wasserstoffdrucks in den Rissen wird über eine Bestimmung des Volumens der Risse und der Menge des Wasserstoffs, der nach der Beladung aus der Probe entgast, realisiert. Das Volumen der Risse wird über die Änderung der Dichte der Probe durch die Wasserstoffbeladung gemessen. Die Messmethode ist in Abschnitt 3.6 beschrieben. Die Proben hatten folgende Probengeometrie:

$$
\begin{aligned}
\text { reines Fe: } & (1 \times 1 \times 0,13) \mathrm{cm}^{3} \\
\text { ARMCO-Fe: } & (1 \times 1 \times 0,1) \mathrm{cm}^{3}
\end{aligned}
$$

Die Ergebnisse der reinen Eisenprobe und der ARMCO-Eisen-Probe sind also gut miteinander vergleichbar (vor allem, weil sich die Risse bei ARMCO-Eisen nur direkt unter der Oberfläche befinden, vergleiche Abbildung 5.40, sodass eine Dickenvariation von zwischen 1 und 1,3 mm keinen starken Einfluss hat). Die Ergebnisse der Dichtemessung sind in den Abbildungen 5.43 und 5.44 gezeigt. Die Balken zeigen jeweils den Fehlerbereich des gemittelten Wertes. Im Falle von reinem Eisen wurden nur die ersten beiden Messwerte berücksichtigt, da danach signifikante Schwankungen auftraten. Die Rissvolumina wurden nach Gleichung (3.3) bestimmt und sind in Tabelle 5.1 zusammengefasst.

\begin{tabular}{|c|c|}
\hline Material & Rissvolumen $\left[\mathrm{mm}^{3}\right]$ \\
\hline reines Fe & $1,80 \pm 0,22$ \\
ARMCO-Fe & $1,96 \pm 0,24$ \\
\hline
\end{tabular}

Tabelle 5.1: Angabe des Gesamtvolumens der Risse.

Die Rissvolumina sind sehr ähnlich, im Rahmen der Messunsicherheit sogar gleich.

Zur Bestimmung des Drucks in den Rissen wird die Probe direkt nach der Beladung in eine Vakuumkammer eingebracht und der Druck gemessen, der sich in der Kammer einstellt. Die Messmethode ist in Abschnitt 3.7 näher beschrieben. In Abbildung 5.45 ist der Druckanstieg in der Vakuumkammer gezeigt. Es wurde während einer Messung wieder auf ein Vakuum von $10^{-5}$ mbar abgepumpt, um die Entgasung der Probe zu beschleunigen. Daher fällt der Druck in der Kammer bei den Messungen ein oder zwei mal auf Null ab. Ist dann nur noch ein geringer Anstieg zu verzeichnen, wurde die Messung beendet. 


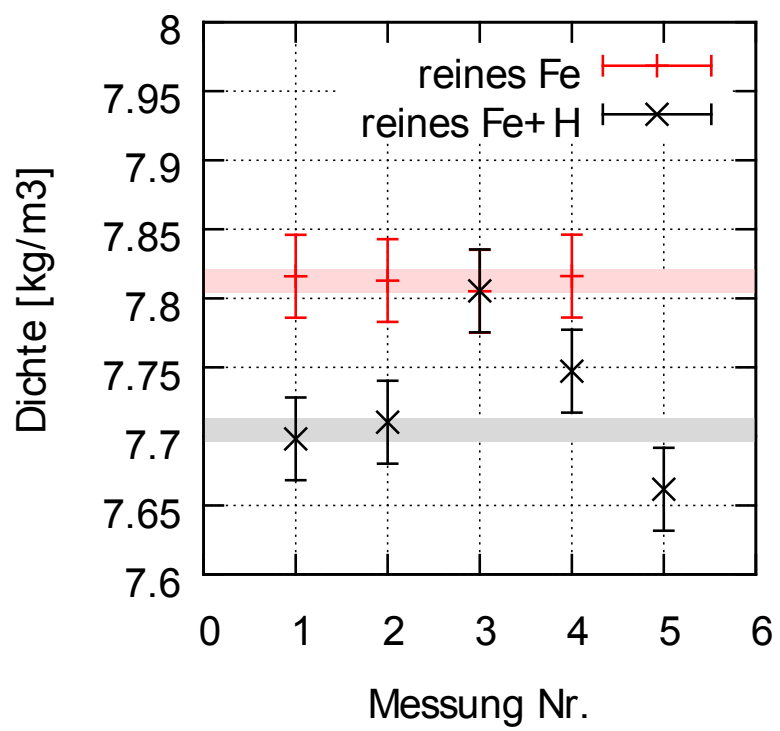

Abbildung 5.43: Vergleich von Dichtemessungen vor und nach der Wasserstoffbeladung an einer reinen Eisenprobe. Die Messungen wurden an unterschiedlichen Tagen durchgeführt, um eine möglichst gute Statistik zu erhalten. Die Dichte der Probe ist durch die Risse im Material verringert.

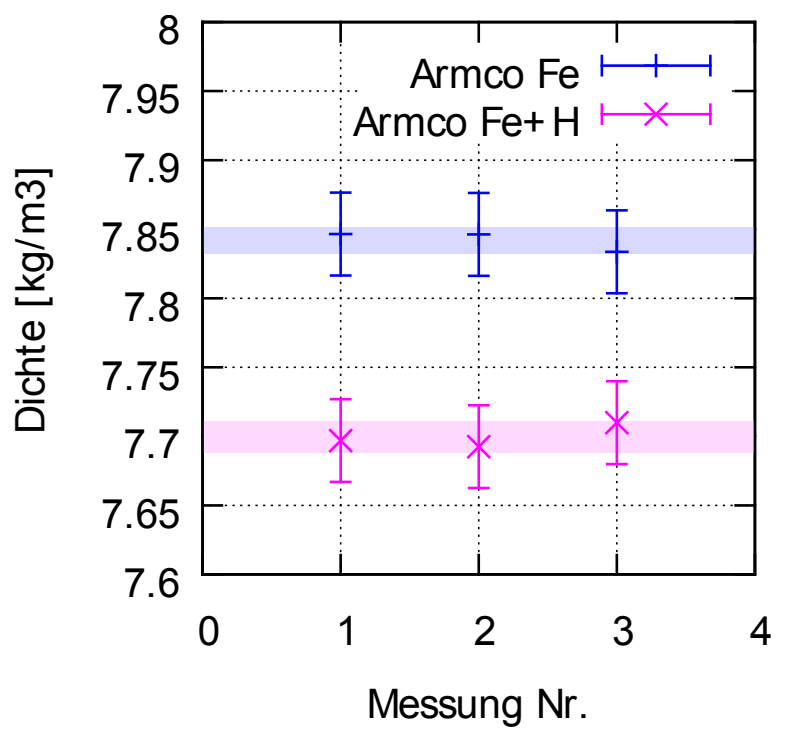

Abbildung 5.44: Vergleich von Dichtemessungen vor und nach der Wasserstoffbeladung an einer ARMCO-Eisenprobe, vergleiche Abbildung 5.43. Im Vergleich zu der reinen Eisenprobe ist die Dichte vor der Beladung leicht höher. Die Dichte nach der Beladung im Rahmen der Messunsicherheit gleich.

Über das ideale Gasgesetz und das Kammervolumen kann die ausgetretene Stoffmenge an Wasserstoff $n_{\text {aktuell }} \mathrm{zu}$ jedem Zeitpunkt $t$ bestimmt werden.

$$
n_{\text {aktuell }}(t)=\frac{p(t) \cdot V_{K}}{R \cdot T}
$$

wobei $p$ der Druck in der Kammer, $V_{K}$ das Volumen der Kammer, $R$ die allgemeine Gaskonstante und $T$ die Temperatur, hier Raumtemperatur, ist. Wird die Kammer zwischendurch abgepumpt, muss die danach ausgetretene Menge addiert werden. Wobei jeweils der letzte gezeigte Anstieg vernachlässigt wird. Man erkennt in Abbildung 5.45, dass bei reinem Eisen dieser Anstieg (bei $\sim 350$ Stunden) noch ein wenig stärker ausfällt, sodass die Stoffmenge hier etwas stärker unterschätzt wird als bei ARMCO-Eisen. Eine untere Grenze für die Stoffmenge wird bei allen Proben durch diese Messmethode angegeben, da man etwa 2 Minuten für den Einbau der Proben in die Kammer benötigt und auch nach der Messung noch minimale Mengen austreten, wie der letzte Anstieg belegt. Der Druck bei Beginn der Messung beträgt:

$$
\begin{aligned}
p_{\text {reines Fe }}(t=0) & =53 \pm 8 \mathrm{MPa} \\
p_{\text {ARMCO-Fe }}(t=0) & =51 \pm 8 \mathrm{MPa}
\end{aligned}
$$




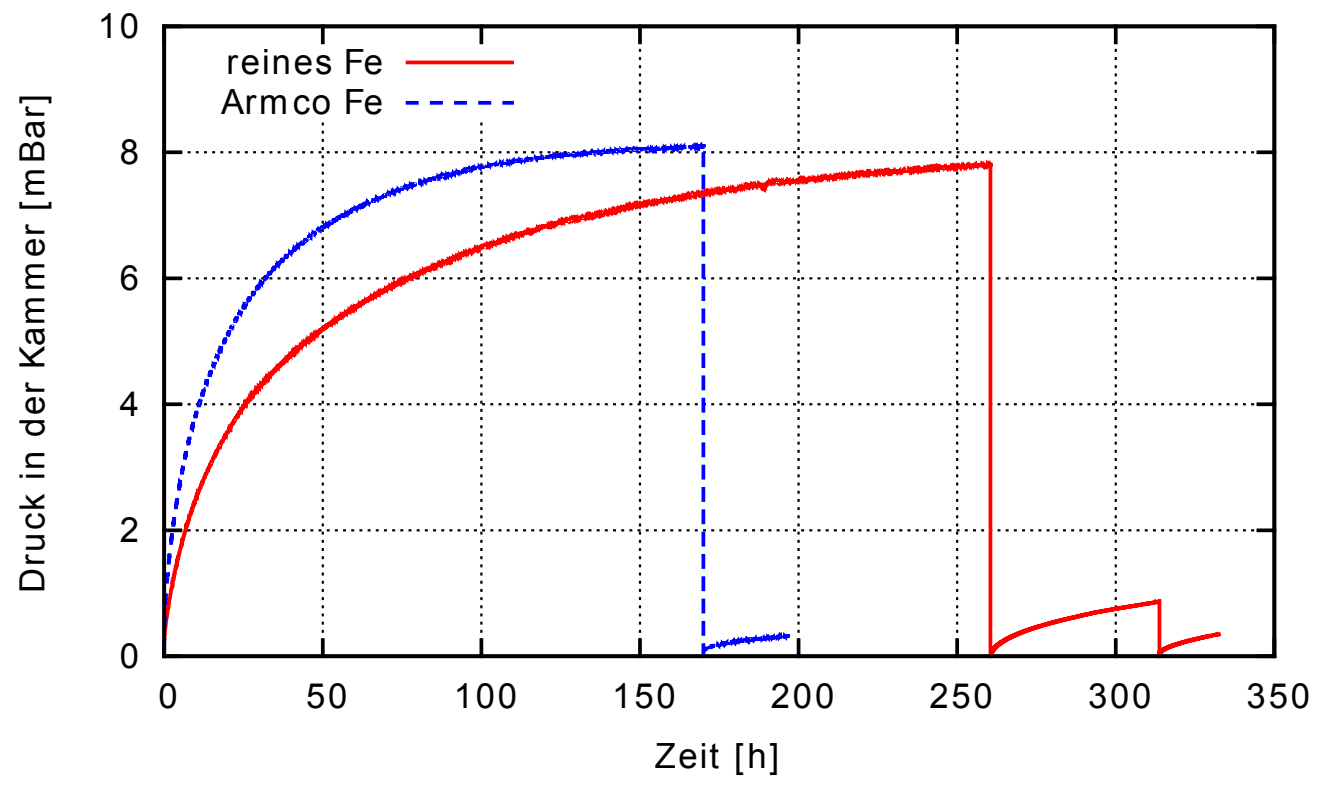

Abbildung 5.45: Messung des Wasserstoffaustritts in ein definiertes Volumen mit $V_{K}=$ $(86,2 \pm 0,9) \mathrm{cm}^{3}$. Der Druck in der Kammer steigt mit der Zeit, da Wasserstoff aus der Probe in die Kammer ausgast. Fällt der Druck innerhalb einer Messung auf Null, wurde abgepumpt um den Austritt des Wasserstoffes zu beschleunigen.

Ein Vergleich der beiden Druckmessungen zeigt eine unterschiedliche Kinetik beim Ausgasen des Wasserstoffs aus der Probe. Im Vergleich zwischen den Proben fällt auf, dass ARMCO-Eisen sehr viel schneller ausgast. Dies liegt an der Verteilung der Risse, denn bei ARMCO-Eisen sind diese, im Gegensatz zu reinem Eisen, nur direkt unter der Oberfläche zu finden.

Der Druck in den Rissen kann über die ausgetretene Stoffmenge am Ende der Messung $n_{\text {Ende }}$, das Rissvolumen $V_{\text {Risse }}$ und den Kammerdruck in Abhängigkeit der Zeit bestimmt werden. Da in den Rissen Drücke im Bereich von einigen $10 \mathrm{MPa}$ erreicht werden, wird zur Berechnung die Van-der-Waals Gleichung für reale Gase und nicht das ideale Gasgesetz verwendet.

$$
p_{\text {Risse }}(t)=\frac{n(t) \cdot R \cdot T}{V_{\text {Risse }}-n(t) \cdot b}-a\left(\frac{n(t)}{V_{\text {Risse }}}\right)^{2}
$$

$n=n(t)=n_{\text {Ende }}-n_{\text {aktuell }}(t)$ ist die Stoffmenge an Wasserstoff in den Rissen, wobei $n_{\text {end }}$ die Menge an Wasserstoff ist, die insgesamt austritt und $n_{\text {aktuell }}$ die zum betrachteten Zeitpunkt ausgetretene Menge. $a, b$ sind die Konstanten der Van-der-Waals Gleichung. Es wurden $a=0,02476 \mathrm{~m}^{6} \mathrm{~Pa} / \mathrm{mol}^{2}$ und $b=2.66110^{-5} \mathrm{~m}^{3} / \mathrm{mol}$ verwendet [AJ06].

Wie schon beim Druckanstieg in der Kammer sieht man in Abbildung 5.46 den deutlich schnelleren Druckabfall in den Rissen von ARMCO-Eisen im Vergleich zu reinem Eisen. Außerdem kann man den Druck direkt nach der Beladung ablesen, welcher in Abbildung 5.46 mit Pfeilen markiert ist. Hierbei ist zu beachten, dass es sich um eine untere 


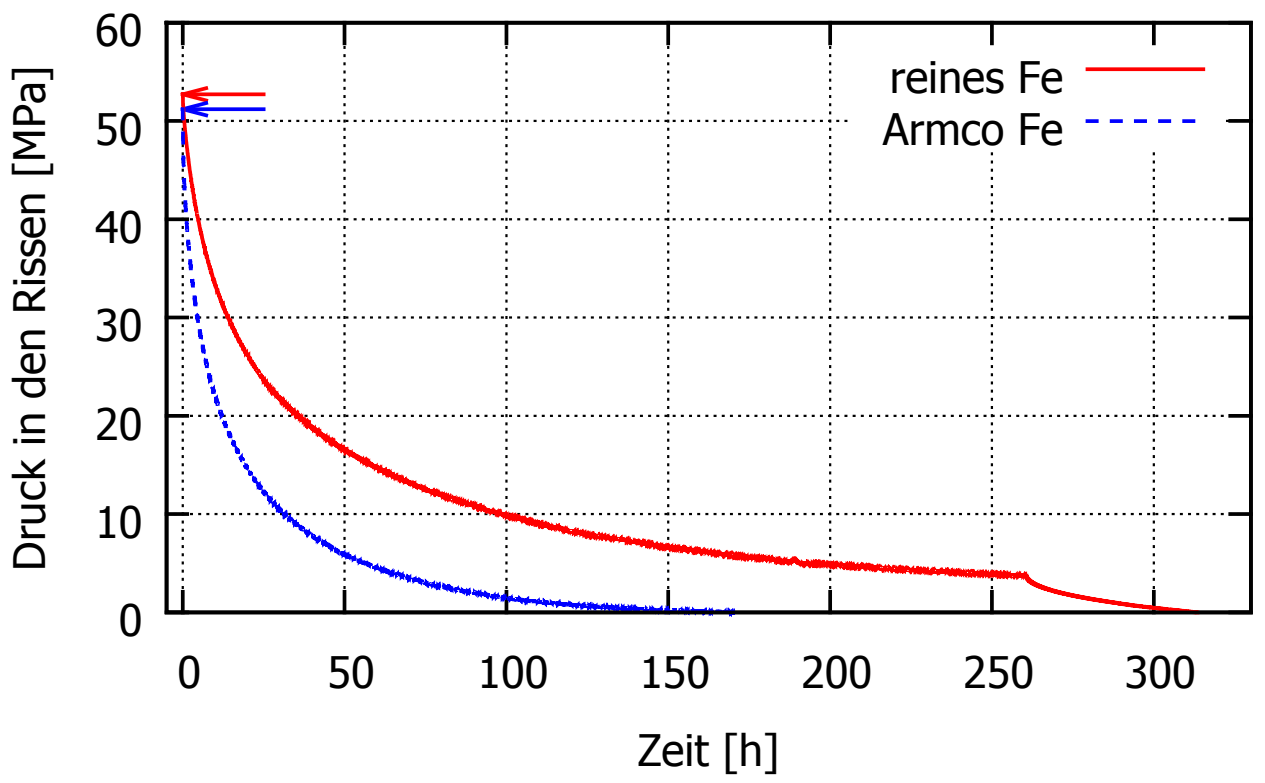

Abbildung 5.46: Druck in den Rissen in Abhängigkeit der Zeit. Startzeitpunkt der Messung ist etwa 2 Minuten nach Ende der Wasserstoffbeladung. Mit Pfeilen ist der Druck zu diesem Zeitpunkt markiert, da dieser eine untere Abschätzung für den Druck in den Rissen während der Beladung angibt.

Abschätzung handelt, da der Einbau der Probe in die Vakuumkammer etwa 2 Minuten dauert und, wie aus Abbildung $5.46 \mathrm{zu}$ erkennen ist, gast der Wasserstoff vor allem am Anfang der Messung schnell aus. Des Weiteren ist zu sehen, dass der Anfangsdruck von ARMCO-Eisen etwa 4,5 MPa niedriger ist als bei reinem Eisen. Dieses entspricht den Erwartungen, denn für Risse direkt unter der Oberfläche muss höchstwahrscheinlich ein geringerer Druck aufgewendet werden als im Inneren des Materials. Allerdings kann dieser niedrigere Druck auch darauf zurückgeführt werden, dass ARMCO-Eisen schneller ausgast und dadurch mehr Wasserstoff bis zum Einbau in die Kammer für die Messung verloren geht.

\section{Wasserstoff Permeation}

Um die Blisterbildung näher zu untersuchen, wurden Permeationsmessungen durchgeführt, bei denen von einer Seite elektrochemisch beladen wird und auf der anderen Seite eine KircTec Sonde die Permeation misst. Der experimentelle Aufbau ist in Abschnitt 3.8 näher beschrieben. Die Beladung erfolgte stufenweise, wobei in den Abbildungen 5.47 und 5.48 die Stromdichte blau gestrichelt eingezeichnet und an der rechten Achse abzulesen ist. Abbildung 5.47 zeigt die Messung an ARMCO-Eisen, welche weniger Rauschen als die Messung an reinem Eisen zeigt. Daher sind die Effekte deutlicher sichtbar. Man beob- 


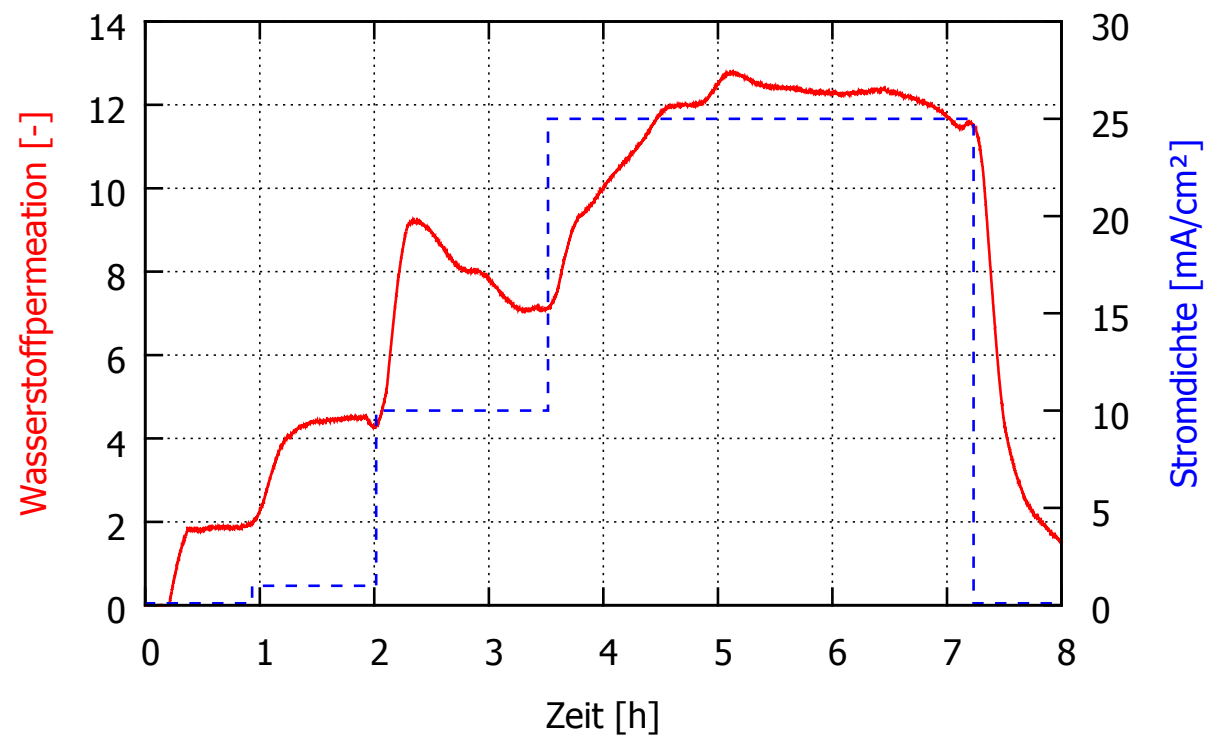

Abbildung 5.47: Permeationsmessung an ARMCO-Eisen. Beladung stufenweise. Stromdichte blau gestrichelt und an rechter Achse abzulesen. Man erkennt in den ersten zwei Beladungsschritten einen Anstieg und eine Sättigung. Bei drittem Beladungsschritt $\left(10 \mathrm{~mA} / \mathrm{cm}^{2}\right)$ ist ein Anstieg und danach ein Abfall zu beobachten.

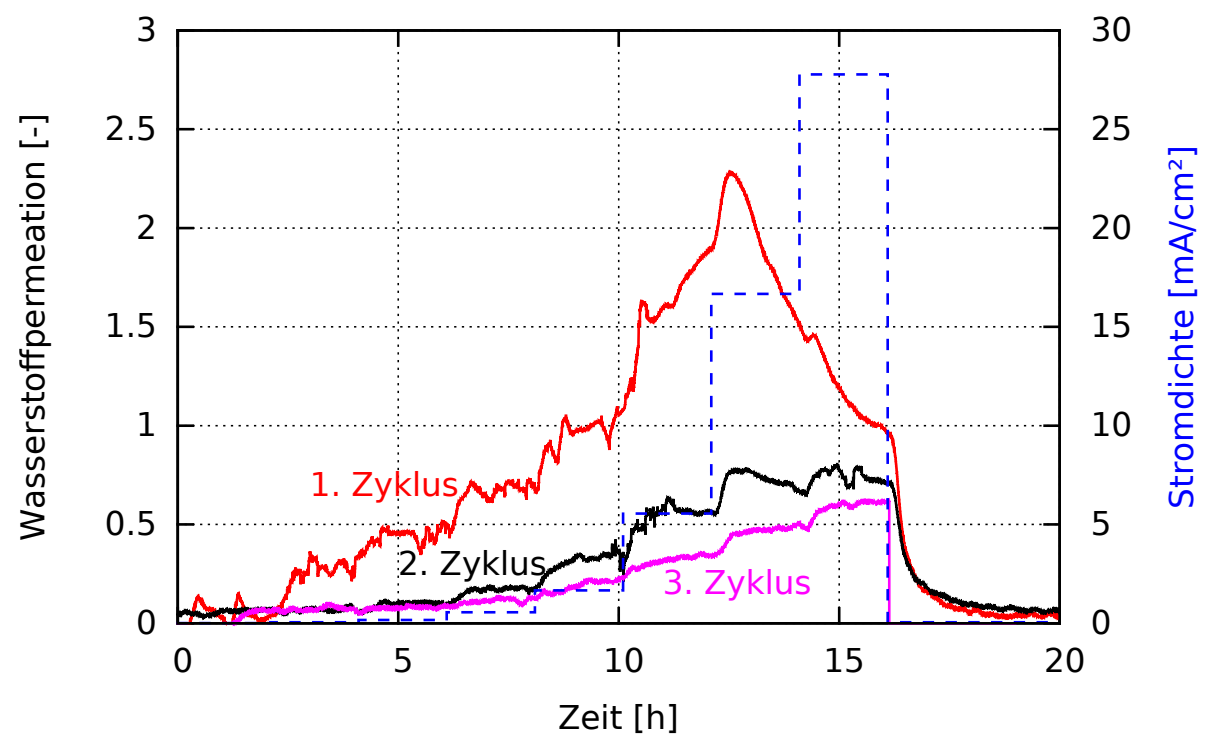

Abbildung 5.48: Permeationsmessung an reinem Eisen. Beladung stufenweise. Stromdichte blau gestrichelt und an rechter Achse abzulesen. Es ist die Wasserstoffpermeation von drei Beladezyklen dargestellt. Die Permeation steigt, wenn die Stromdichte erhöht wird. Bei einer Stromdichte von $17 \mathrm{~mA} / \mathrm{cm}^{2}$ ist ein Abfall der Permeation zu beobachten.

achtet in den ersten beiden Beladestufen $\left(0,1 \mathrm{~mA} / \mathrm{cm}^{2}\right.$ und $\left.1 \mathrm{~mA} / \mathrm{cm}^{2}\right)$ einen Anstieg in der Wasserstoffpermeation, die in eine Sättigung übergeht. Bei der folgenden Beladestufe von $10 \mathrm{~mA} / \mathrm{cm}^{2}$ folgt auf den Anstieg direkt ein Abfall in der Wasserstoffpermeation. Es 
handelt sich bei dieser Beladestromdichte um den Wert bei dem auch erste Blister zu erwarten sind, vergleiche Abschnitt 5.1.8. Das bedeutet, dass die Permeation durch die Blister deutlich herabgesetzt wird, da der Wasserstoff in ihnen getrappt wird. Das gleiche Phänomen ist auch für reines Eisen, Abbildung $5.48 \mathrm{zu}$ beobachten. Hier wird ein deutlicher Abfall beim Erhöhen innerhalb der Beladestufe von $17 \mathrm{~mA} / \mathrm{cm}^{2}$ registriert (Beladestufen sind anders als bei ARMCO-Eisen gewählt). Dies erklärt nun auch, dass die Risse bei ARMCO-Eisen nicht im Inneren sondern nur unter der Oberfläche lokalisiert sind. Es bilden sich bei ARMCO-Eisen so viele Risse direkt unter der Oberfläche, dass der Wasserstoff im Inneren der Probe nicht mehr in ausreichend großer Konzentration vorliegt, damit sich Risse bilden können. Bei reinem Eisen scheint es direkt unter der Oberfläche nicht genügend Initiierungsstellen zu geben, sodass der Wasserstoff in hoher Konzentration tiefer ins Material gelangt. Dies entspricht den Erwartungen für ein Material mit höherer Reinheit.

\section{Zugversuche $\quad 5.1 .11$}

Es wurde in Abschnitt 5.1.9 der Wasserstoffdruck in den Rissen bestimmt. Um diesen mit den mechanischen Kennwerten vergleichen zu können, wurden die Streckgrenze und die Zugfestigkeit der Proben mit Zugversuchen ermittelt. Darüber hinaus interessiert, wie sich das mechanische Verhalten durch den gelösten Wasserstoff und die Risse in der Probe verändert, daher wurde auch ein Zugversuch direkt nach der Wasserstoffbeladung durchgeführt. Die gezeigten Messungen wurden im Rahmen der Bachelorarbeit von Jonas Wawra [Waw16] durchgeführt und die Messdaten sind dieser Arbeit entnommen.

\begin{tabular}{c|c|c|c|c} 
& \multicolumn{2}{|c|}{ reines Eisen } & \multicolumn{2}{c}{ ARMCO-Eisen } \\
& unbeladen & beladen & unbeladen & beladen \\
\hline Streckgrenze $[\mathrm{MPa}]$ & 65 & 65 & 70 & 75 \\
Elastizitätsgrenze $R_{p 0,2}[\mathrm{MPa}]$ & 131 & 115 & 113 & 166 \\
Zugfestigkeit $[\mathrm{MPa}]$ & 254 & 205 & 257 & 250
\end{tabular}

Tabelle 5.2: Mechanische Kennwerte der Eisenproben vor und nach Wasserstoffbeladung. Abgelesen aus den Zugexperimenten, vergleiche Abbildungen 5.49 und 5.50. Die Werte für ARMCOEisen sind ebenfalls abgelesen aus einem Spannung-Dehnungsdiagramm, gezeigt im Anhang in Abbildung A.4.

Die Kennwerte sind in Tabelle 5.2 zusammengefasst. Die Streckgrenze ist schwer abzulesen, daher wurde zusätzlich die Elastizitätsgrenze bestimmt, bei welcher unter Entlastung 


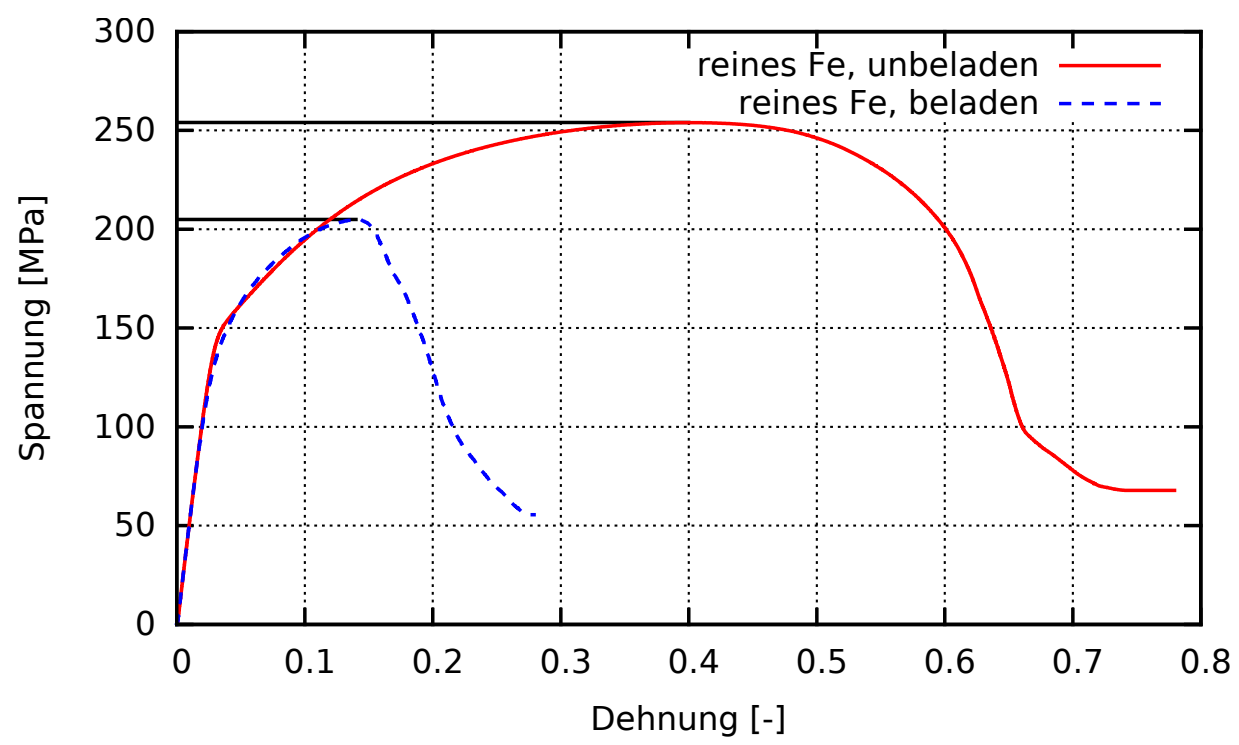

Abbildung 5.49: Zugversuch von reinem Eisen vor und nach Wasserstoffbeladung. Die schwarzen Linien kennzeichnen die gemessenen Zugfestigkeiten. Messung durchgeführt von Jonas Wawra [Waw16].

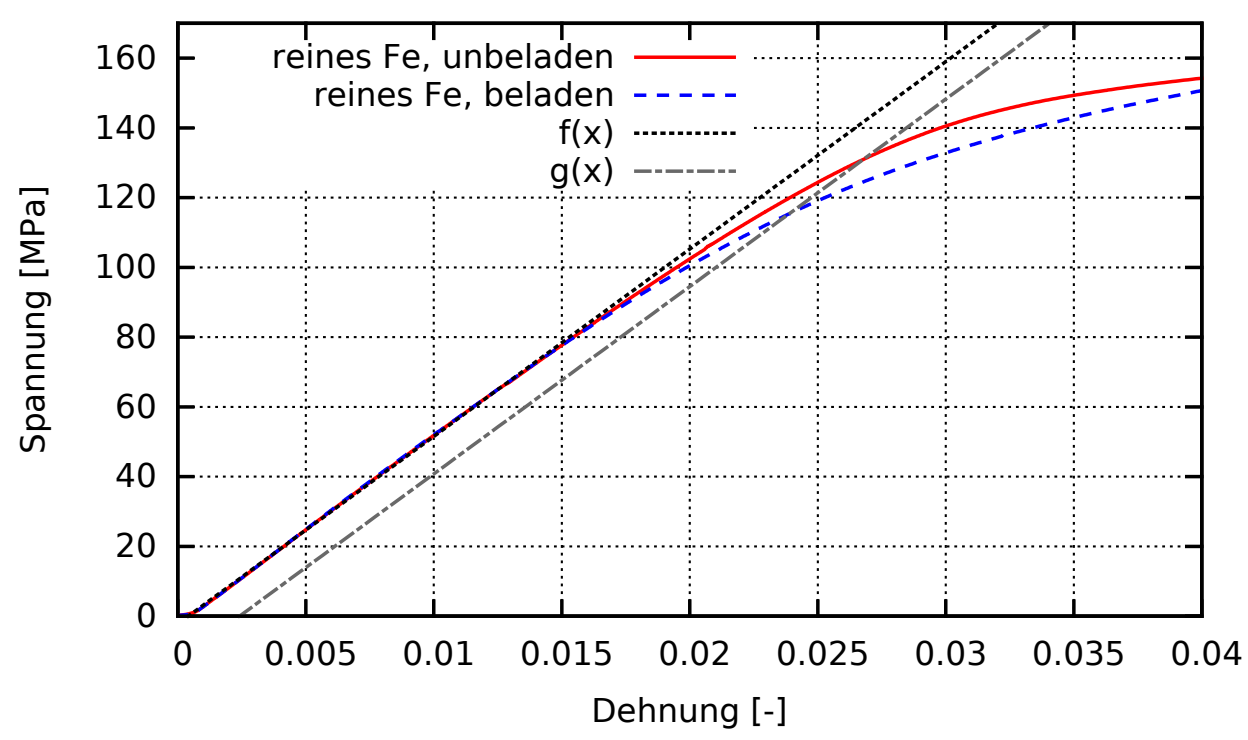

Abbildung 5.50: Vergrößerung von Abbildung 5.49 zur Ermittlung der Streckgrenze. f(x) stellt einen Fit an den linearen Bereich da. $\mathrm{g}(\mathrm{x})$ ist definiert durch $\mathrm{g}(\mathrm{x})=\mathrm{f}(\mathrm{x}-0,002)$ und dient zur Ermittlung der Dehngrenze $R_{p 0,2}$. Messung durchgeführt von Jonas Wawra [Waw16].

der Probe eine Restdehnung von 0,2\% bestehen bleiben würde. Die Streckgrenze ist bei den verwendeten Eisenproben zwischen 65 und $75 \mathrm{MPa}$, wobei sie für das reinere Eisen niedriger ist. Dieses ist zu erwarten, da Fremdatome Versetzungen pinnen können. Es gibt keinen signifikanten Einfluss von Wasserstoff auf die Streckgrenze. Ein anderes Bild ergibt sich allerdings, wenn man die Elastizitätsgrenze $R_{p 0,2}$ betrachtet. Hier scheint es 
für reines Eisen durch Wasserstoff eine Absenkung und für ARMCO-Eisen eine Erhöhung der Elastizitätsgrenze zu geben. Die Zugfestigkeit ist bei beiden Eisen-Proben durch den gelösten Wasserstoff beziehungsweise durch die Risse herabgesetzt, wobei dieser Effekt bei reinem Eisen sehr viel deutlicher auftritt.

\section{Finite-Elemente Simulation der Spannung um Risse}

\section{Spannungsintensitätsfaktor}

In Abschnitt 5.1.9 wurde der Wasserstoffdruck in den Rissen nach der Beladung gemessen. Zur Beurteilung, welche Spannungen dadurch im Material induziert werden, wurden Simulationen mit einer Finite-Elemente-Methode durchgeführt. Die Probengröße war jeweils $1 \mathrm{~mm} \times 1 \mathrm{~mm}$. Im Zentrum der Probe war ein elliptisches Loch, dessen kleine Halbachse $5 \mu \mathrm{m}$ groß ist. Die große Halbachse wurde von 5 bis $100 \mu \mathrm{m}$ variiert. Dieses elliptische Loch soll einen Riss im Material simulieren. Die Geometrie orientiert sich dabei an den Rissen, die im Querschnitt der Probe beobachtet wurden, vergleiche Abbildung 5.11. Der Krümmungsradius $\rho_{t}$ der Rissspitze ist bei einer Ellipse gegeben durch:

$$
\rho_{t}=b^{2} / a
$$

Im Riss wurde ein Druck von 50 MPa eingestellt. Das entspricht dem in Abschnitt 5.1.9 gemessenen Druck in den Rissen.

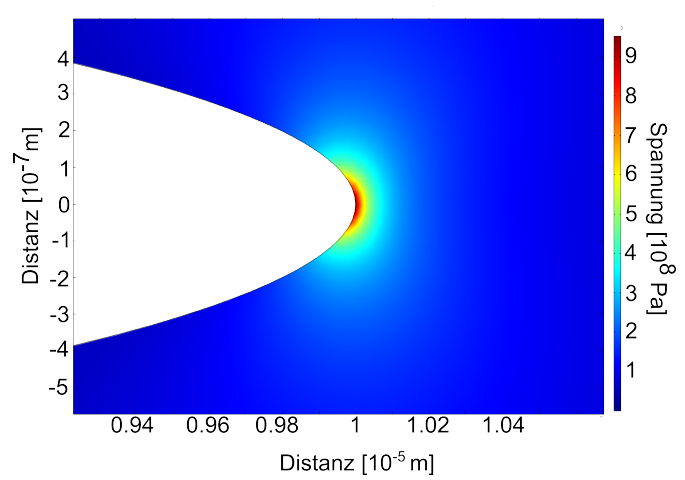

Abbildung 5.51: FEM-Simulation erstellt in Zusammenarbeit mit Niklas Teichmann. Spannungsverteilung um eine Rissspitze in Eisen. Der Druck innerhalb des Risses beträgt $50 \mathrm{MPa}$.

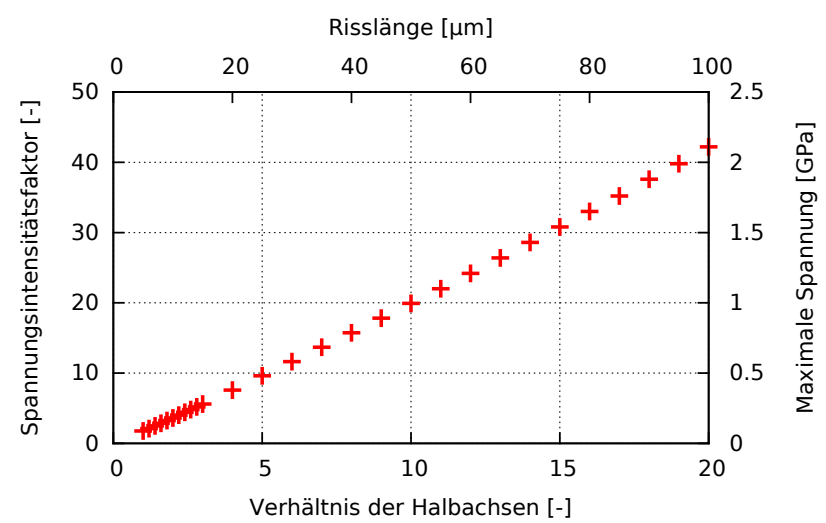

Abbildung 5.52: FEM-Simulation erstellt in Zusammenarbeit mit Niklas Teichmann. Auftragung der maximalen Spannung in Eisen mit elliptischem Riss. Der Druck innerhalb des Risses beträgt $50 \mathrm{MPa}$. 
In Abbildung 5.51 ist das Spannungsfeld um den Riss dargestellt. Man erkennt eine maximale Spannung vor der Rissspitze von etwa $1 \mathrm{GPa}$. Wie erwartet gibt es vor einer Rissspitze eine Spannungsintensivierung, vergleiche Abschnitt 2.2.3. Der Spannungsintensitätsfaktor ist gegeben durch die maximal im Material induzierte Spannung im Verhältnis zum Druck im Riss, der hier immer $50 \mathrm{MPa}$ ist. Er ist in Abbildung 5.52 in Abhängigkeit des Halbachsenverhältnisses beziehungsweise der Risslänge dargestellt. Man erkennt, dass eine lineare Abhängigkeit besteht. In Abschnitt 2.2.3 wurde der Spannungsintensitätsfaktor für ein Material mit einem Riss unter Zugbelastung hergeleitet. Setzt man in Gleichung (2.10) den Krümmungsradius einer Ellipse ein, Gleichung (5.7), ergibt sich ebenfalls eine lineare Abhängigkeit.

Aus dieser Simulation kann geschlossen werden: In einem runden Loch muss ein etwa 40 mal so großer Druck wie in einem $100 \mu \mathrm{m}$ langen Riss vorliegen, um die gleiche Spannung im Material zu induzieren. In einem $100 \mu \mathrm{m}$ langen Riss wird so eine Spannung von circa 2 GPa im Material induziert, wenn $50 \mathrm{MPa}$ Gasdruck im Riss vorliegt. Der experimentell gemessene Druck entspricht dabei einem Riss mit maximaler Länge, da nach der Beladung Risse mit Längen im Bereich $100 \mu \mathrm{m}$ vorliegen. Bei der Rissinitiierung liegt der Druck zwischen einem runden Einschluss und der umgebenden Eisenmatrix vor. Dieses kann durch ein rundes Loch beschrieben werden. Das bedeutet, dass für die Rissinitiierung ein etwa 40 mal höherer Druck vorliegen muss, damit die gleiche Spannung im Material um den Riss beziehungsweise das Loch vorliegt. Man kann daher vermuten, dass auch der Druck in einem runden Loch etwa 40 mal so hoch sein muss wie der gemessene Druck, damit es zur Rissinitiierung kommt. Diese Schlussfolgerung wird in Abschnitt 6.5 mit Messungen verglichen und diskutiert. 


\section{\begin{tabular}{l|r} 
Simulation eines oberflächennahen Risses & 5.2 .2
\end{tabular}}

Eine weitere Simulation ist in den Abbildungen 5.53 und 5.54 gezeigt. Anstatt in der Mitte der Probe ist der Riss nun in einer Entfernung von $100 \mu \mathrm{m}$ von der Oberfläche des Material positioniert. Das Spannungsfeld ist in Abbildung 5.53 dargestellt. Man erkennt eine deutliche Spannungsintensivierung vor der Rissspitze. Außerdem kann man eine erhöhte Spannung an der Oberfläche mittig über dem Riss und zwischen Rissspitze und Oberfläche beobachten. In Abbildung 5.54 ist das Verschiebungsfeld dargestellt. Hier kann man erkennen, dass sich das Material zur Oberfläche hin verschiebt.

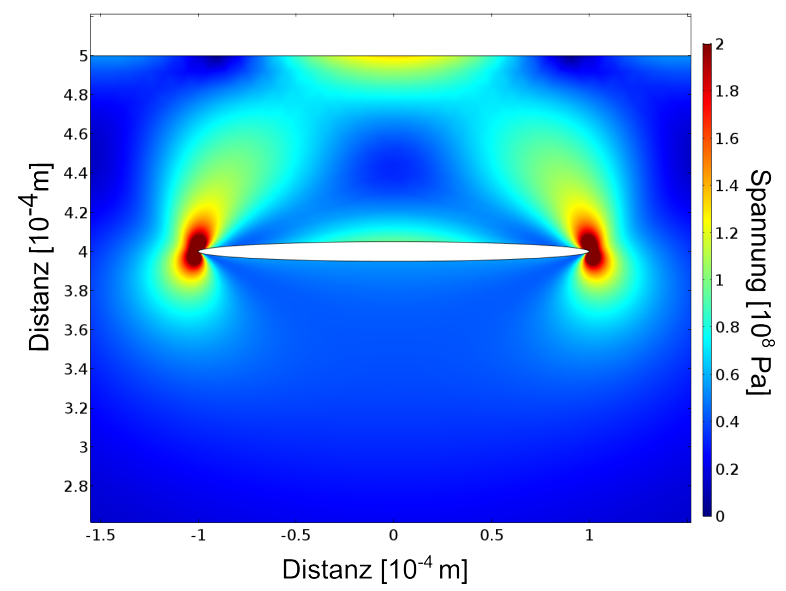

Abbildung 5.53: FEM-Simulation erstellt in Zusammenarbeit mit Niklas Teichmann. Spannungsverteilung um einen oberflächennahen Riss. Druck innerhalb des elliptischen Risses beträgt $50 \mathrm{MPa}$.

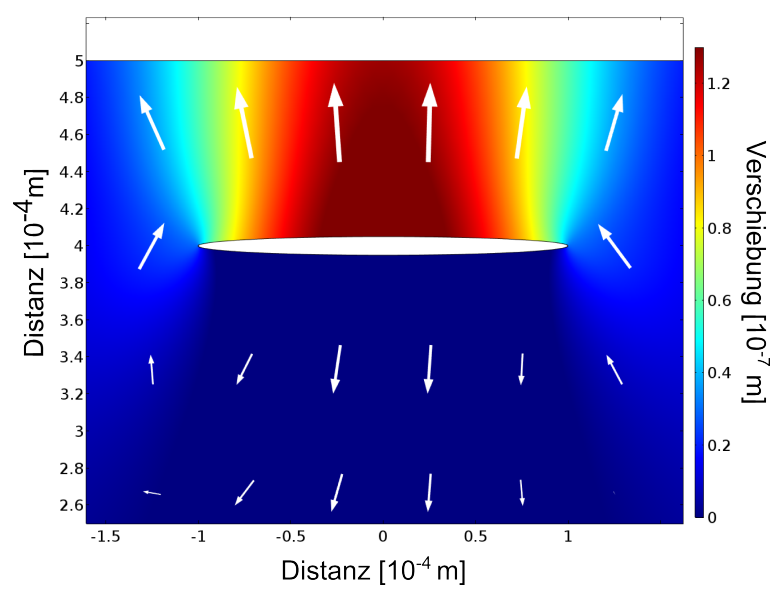

Abbildung 5.54: FEM-Simulation erstellt in Zusammenarbeit mit Niklas Teichmann. Verschiebungsfeld um einen oberflächennahen Riss. Druck innerhalb des Risses beträgt $50 \mathrm{MPa}$. Die weißen Pfeile kennzeichnen die Richtung des Verschiebungsfeldes. Die Länge der Pfeile gibt die logarithmische Intensität des Feldes in an. 


\section{\begin{tabular}{l|l} 
& \\
Diskussion & 6
\end{tabular}}

In diesem Abschnitt sollen die Ergebnisse zur Rissbildung in Eisen kritisch diskutiert und mit der bestehenden Literatur verglichen werden. Dabei werden die Ergebnisse nicht, wie in Abschnitt 5, nach den verwendeten experimentellen Methoden sortiert, sondern es erfolgt eine Ordnung, die sich an der Abfolge der physikalischen Ereignisse der Rissbildung orientiert:

- Zunächst wird Rissinitiierung an nichtmetallischen Einschlüssen beschrieben, siehe Abschnitt 6.1. Die chemische Charakterisierung ist in Abschnitt 6.2 vorgestellt. Es wird darauf eingegangen, wie sich die Verteilung der Risse bei einer Änderung der Reinheit des Materials verhält, siehe Abschnitt 6.3. In Abschnitt 6.4 erfolgt eine thermodynamische Beschreibung, warum Einschlüsse Initiierungsstellen bilden.

- Der hohe Wasserstoffdruck in den Rissen wird quantifiziert und als Triebkraft für die Rissbildung identifiziert, siehe Abschnitt 6.5.

- In den Abschnitten 6.6 und 6.7 wird der Rissfortschritt durchs Material beschrieben und der Einfluss der Wasserstoffversprödung diskutiert.

- Die Defektstruktur um Risse und Blister wird im Rahmen des HELP-Mechanismus diskutiert, vergleiche Abschnitt 6.8.

\section{Initiierungsstellen der Wasserstoff induzierten- Risse

Eine Motivation, die Rissbildung in hochreinem Eisen zu untersuchen, bestand darin, den Prozess so detailliert und generell wie möglich zu verstehen. Dafür ist es nützlich, ein möglichst einfaches Materialsystem zu wählen, daher wurde reines Eisen verwendet. Bei der gegebenen Zusammensetzung, vergleiche Tabelle 4.1, wurde zunächst davon ausgegangen, dass Einschlüsse im Material nicht vorhanden, beziehungsweise sehr selten sind. In der Literatur werden als Initiierungsstellen häufig Leerstellencluster vorgeschlagen [RZC $\left.{ }^{+} 07\right]$. Zur Überprüfung dieser Hypothese wurde das beschriebene möglichst reine Eisen als Materialsystem gewählt. Die Proben wurden elektrochemisch mit Wasserstoff beladen. Dazu wurde ein saurer Elektrolyt mit einem Wasserstoffpromoter verwendet. Die Beladung erfolgte bei konstantem Strom, vergleiche Abschnitt 3.1. Nach dieser Wasserstoffbeladung sind die Proben mit Rissen durchzogen, siehe Abbildung 5.11. Um die Initiierungsstellen 
der Risse zur Beobachtung freizulegen, wurde mit den beladenen Proben ein Zugversuch bis zum Bruch durchgeführt. Der Bruch der Probe findet durch ein Anwachsen der Risse in Richtung senkrecht zur Zugrichtung und schließlich eine Verbindung dieser Risse statt. Somit werden die Bruchflächen der Wasserstoff-induzierten Risse zur Beobachtung freigelegt. In einer Analyse der Bruchfläche konnten die Initiierungsstellen der Wasserstoffinduzierten Brüche ausgemacht werden. Jeweils im Zentrum einer Rissfläche, die durch eine meist konzentrische Geometrie zu erkennen ist, befindet sich ein Loch mit einem Durchmesser von einigen Mikrometern. In etwa 50\% der Fälle ist in diesem Loch ein Einschluss zu finden. Im Fall eines Loches ohne Einschluss ist dieser auf dem Gegenstück der Bruchprobe zu finden. Es konnte kein Riss identifiziert werden, der nicht an einem Einschluss initiiert wurde. Das bedeutet, dass trotz der Verwendung eines hochreinen Eisens, lediglich Einschlüsse als Rissinitiierungsstellen ausgemacht werden konnten. Risse, die nahe der Oberfläche liegen, verursachen Blister auf der Oberfläche. Auf der Oberfläche über einem Riss wird Probenmaterial aufgeworfen, siehe Abbildung 5.5. Risse, die einen Blister verursachen, liegen oft parallel zur Oberfläche, daher werden diese bei der Betrachtung der Bruchfläche nach dem Zugversuch nicht analysiert, denn die Öffnung von Rissen, die parallel zur Probenoberfläche verlaufen, ist unwahrscheinlich. Darüber hinaus liegt die Bruchfläche, falls sie geöffnet wird, nicht in der Bildebene. Somit werden bei Untersuchungen der Bruchflächen nach Zugversuchen vor allem diejenigen Risse ausgewertet, die tief im Material liegen und senkrecht zur Probenoberfläche verlaufen, diese wurden stets an Einschlüssen initiiert. Es gibt Abschnitte auf der Bruchfläche, die nicht klar einem Riss zugeordnet werden können, sodass nicht mit absoluter Sicherheit ausgeschlossen werden kann, dass es auch andere Initiierungsstellen für Risse im reinen Eisen gibt. Die Vielzahl der Risse, die eindeutig zu identifizieren waren, werden jedoch alle an Einschlüssen initiiert. Ein ähnliches Verfahren zur Betrachtung der Initiierungsstellen, wenngleich deutlich komplizierter, kam in den Arbeiten von Ren et al. [ $\left.\mathrm{RCL}^{+} 08, \mathrm{RZS}^{+} 08\right]$ zum Einsatz, um Bruchflächen freizulegen. In ihrer Arbeit wurden die beladenen Proben zwischen zwei Platten aus einer $\mathrm{CuAg}$ Legierung eingebettet und beim auseinanderbiegen dieser konnten oberflächennahe Risse geöffnet werden. Die Autoren beobachteten, dass $88 \%$ aller Risse an Einschlüssen initiiert werden. Ren et al. $\left[\mathrm{RCL}^{+} 08\right]$ schlägt als Initiierungsmechanismus für Risse, die nicht an Einschlüssen entstehen, eine Ansammlung von Leerstellen vor. Eine erhöhte Konzentration von Leerstellen bei Wasserstoffbeladung konnte in früheren Arbeiten nachgewiesen werden [Fuk03, SF14]. Allerdings ist ein direkter Nachweis für Rissinitiierung an Leerstellenclustern schwierig. Im Vergleich dazu konnten in dieser Arbeit tief im Material nur Einschlüsse als Initiierungsstellen identifiziert werden, wobei auf oberflächennahe Risse im Folgenden noch genauer eingegangen wird.

Neben Einschlüssen können auch planare Defekte durch Kaltwalzen oder Grenzflächen zu einer zweiten Phase oder innerhalb der zweiten Phase (zum Beispiel Zementit in Stahl) Initiierungsstellen sein $\left[\mathrm{SSR}^{+} 13, \mathrm{KTA}^{+} 14, \mathrm{BDK} 09\right]$. Das Auftreten anderer eisenhaltiger 
Phasen kann bei den untersuchten Eisenproben aufgrund der hohen Reinheit ausgeschlossen werden. Die Initiierung an planaren Defekten kann als Ursprung für in dieser Arbeit beobachteten Multiblistern vermutet werden, vergleiche Abbildung 5.8 und 5.12. Bei Multiblistern handelt es sich um meist kleine Blister, die auf der Flanke eines großen Blisters zu finden sind. Im Querschnitt ist ein langer Riss, der den großen Blister auf der Oberfläche verursacht, zu beobachten. Zwischen diesem großen Riss und der Probenoberfläche finden sich kleine Risse, im Falle von Abbildung 5.12 sind zwei zu erkennen. Diese verursachen zwei kleinere Blister auf den Flanken des großen Blisters. Eine Entstehung von Multiblistern durch zufällige Häufung von einzelnen Blistern kann aufgrund der hohen Anzahl von Multiblistern ausgeschlossen werden. Es wird daher davon ausgegangen, dass ein bestehender Blister die Bildung von weiteren begünstigt. Ebenfalls wird es als eher unwahrscheinlich eingestuft, dass zur Initiierung der kleinen Risse jeweils ein Einschluss Ausgangspunkt ist, da es sich um ein sehr reines Material handelt; ausgeschlossen werden kann dies allerdings nicht. Die Initiierung müsste dann an Defekten stattfinden, die durch den großen Riss und den zugehörigen Blister auf der Oberfläche vorliegen. In Querschnittslamellen, die in Abschnitt 6.8 noch näher diskutiert werden, wurden Scherbänder beobachtet, vergleiche Abbildung 5.32. Hier liegt höchstwahrscheinlich eine erhöhte Wasserstoffkonzentration vor, da der Wasserstoff an der Vielzahl der Versetzungen, die die Scherbänder bilden, getrappt werden kann [KJ80, DBC $\left.{ }^{+} 15\right]$. Es kann daher vermutet werden, dass es auch ohne einen Einschluss zur Rissinitiierung kommen kann. Die Rissbildung ist hier lokal vergleichbar mit Proben, die vorher einer massiven Verformung ausgesetzt wurden, wie zum Beispiel kalt gewalzten Proben. Solche Experimente wurden von Laureys et al. [LPV16] an sehr kohlenstoffarmem Stahl durchgeführt. Sie beobachteten, dass in einer kalt gewalzten Probe deutlich mehr Blister gebildet werden als in einer rekristallisierten bei gleicher Wasserstoffbeladung. Dies bestätigt die Vermutung, dass die Blisterbildung durch eine hohe Defektdichte begünstigt wird. Im Rahmen der vorliegenden Arbeit konnte eine erhöhte Defektdichte im Material lokal um Risse beobachtet werden, vergleiche Abbildung 5.36. Besonders zwischen Rissen und der Probenoberfläche entstehen wegen der erhöhten lokalen Wasserstoffkonzentration daher sekundäre Risse, die zu Multiblistern auf der Oberfläche führen. Diese Schlussfolgerung widerspricht nicht der Beobachtung, dass auf der Bruchfläche der Probe nach einem Zugversuch nur Risse, die an Einschlüssen initiiert wurden, vorlagen. Denn bei diesen handelt es sich, wie oben beschrieben, um Risse tief in der Probe. Im Querschnitt der Probe, vergleiche Abbildung 5.11, wurden sekundäre Risse, die zu Multiblistern führen können, nur oberflächennah beobachtet. Somit kann die Schlussfolgerung gezogen werden, dass in hochreinem und gut ausgelagerten Eisen Risse zunächst an Einschlüssen initiiert werden, danach können sekundäre Risse in stark verformtem Material zwischen Rissen und der Probenoberfläche entstehen. Im Inneren des Materials ist eine Bildung von sekundären Rissen in direkter Nähe zu primären Rissen möglich, wird aber als deutlich unwahrscheinlicher eingestuft. 
Denn wie später in Abschnitt 6.3 diskutiert wird, ist die Wasserstoffkonzentration im Inneren der Probe nach der Bildung von primären Rissen sehr viel niedriger. Direkt unter der Oberfläche, liegt jedoch weiter eine hohe Wasserstoffkonzentration durch die Beladung vor.

Daraus kann für die Anwendung von Eisen in der Industrie geschlossen werden, dass sich eine starke Verformung, zum Beispiel durch Walzen der Werkstoffe, negativ auf die Widerstandsfähigkeit der Materialien gegenüber Wasserstoff-induzierter Rissbildung auswirken kann. Ebenso kann eine Verformung von Eisenwerkstoffen während der Benutzung diesen Effekt haben.

\section{Chemische Zusammensetzung und Form der Einschlüsse}

Die chemische Zusammensetzung der Einschlüsse wurde mit EDX gemessen, vergleiche Abbildung 5.18 und 5.19. Es wurde jeweils ein Punktscan auf dem Einschluss und einer in direkter Nähe (etwa 10-20 $\mu \mathrm{m}$ entfernt) als Hintergrundscan aufgenommen. So kann ausgeschlossen werden, dass nachgewiesene Elemente von einer Verschmutzung der Probenoberfläche herrühren. Der Hintergrundscan zeigte immer nur reines Eisen mit einer leichten Kontamination mit Kohlenstoff; diese ist für EDX-Messungen typisch. In den EDXMessungen der Einschlüsse wurde immer eine erhebliche Menge Sauerstoff nachgewiesen. Dieses kann nicht auf eine Oxidation der Probe zurückgeführt werden, da Sauerstoff im Hintergrundscan nicht gemessen wurde. Es handelt sich somit bei den Einschlüssen um Oxide. Darüber hinaus wurde in nahezu allen Messungen Mangan, Aluminium und Silizium nachgewiesen. Das bedeutet, dass es sich um Mangan-, Aluminium- und Siliziumoxid handelt. Im Vergleich mit der Literatur, zum Beispiel mit Elboujdaini und Revie [ER09], werden Wasserstoff-induzierte Brüche häufig mit Mangansulfid-Ausscheidungen in Verbindung gebracht, aber auch von Titan-, Aluminium- und Siliziumoxid-Ausscheidungen werden als Initiierungsstellen in Eisen berichtet, wobei Titanoxid nach Mangansulfid am häufigsten gefunden wird, vergleiche Ren et al. [RCL $\left.{ }^{+} 08\right]$. Die Tatsache, dass in dieser Arbeit kein Mangansulfid und auch kein Titanoxid in den Ausscheidungen nachgewiesen wurde, liegt höchstwahrscheinlich an der Reinheit des Materials. Somit kann eine leicht geringere Anfälligkeit des in dieser Arbeit verwendeten Materials gegenüber Rissbildung durch Wasserstoff im Vergleich zu Eisen, das diese Elemente enthält, vermutet werden. Diese kann allerdings nicht näher quantifiziert werden und wie gezeigt wurde, kommt es trotzdem zur Rissbildung im Material. Um Wasserstoff-induzierte Risse in jedem Fall zu vermeiden, ist also eine Reduzierung von Fremdatomen keine ausreichende Maßnahme, wenngleich eine Reduzierung der Anfälligkeit vor allem durch die Herabsetzung des Mangan- und Schwefelgehalts oft als mögliche Maßnahme angesehen, allerdings auch kontrovers diskutiert wird [DAOG01]. Die Anfälligkeit des Materials mit unterschiedli- 
chen Einschlüssen gegenüber Rissbildung durch Wasserstoff kann Gegenstand zukünftiger Arbeiten sein. Weiter konnte durch die Untersuchungen dieser Arbeit gezeigt werden, dass die Verwendung eines besonders reinen Materials nicht dazu führt, dass die Risse an Hohlräumen zum Beispiel durch Leerstellenagglomeration initiieren. Die Bildung von Leerstellen-Clustern unter Wasserstoffbeladung und einer anschließenden Rissinitiierung an diesen wird von einigen Autoren als Initiierungsprozess vorgeschlagen, vergleiche [CS93, RZC ${ }^{+}$07]. Dieser Mechanismus wird in Eisen aufgrund der vorliegenden Ergebnisse als unwahrscheinlich eingestuft, da eine vollständige Entfernung von Fremdatomen aus Eisen nicht möglich ist und, offensichtlich auch bei einer geringen Dichte an Einschlüssen, diese die Schwachstellen des Materials gegenüber der Rissbildung darstellen. Allerdings kann, wie oben beschrieben, auch eine erhöhte Defektdichte, die um Risse auftritt, zur Initiierung von Rissen führen, wie es bei Multiblistern der Fall ist.

Als nächstes soll die Form der Einschlüsse näher betrachtet werden. Die Einschlüsse sind rund und haben einen Durchmesser von 1-5 $\mu \mathrm{m}$. Im Gegensatz dazu wird in der Literatur von längliche Einschlüsse als Ausgangspunkt für Risse berichtet $\left[\mathrm{RCL}^{+} 08\right.$, STTY11, ER09]. Die längliche Form führt zu einer Spannungskonzentration. Diese länglichen Einschlüsse, meist Mangansulfid, treten vor allem bei gewalztem Eisen und Stahl auf. Dass in der vorliegenden Arbeit nur runde Einschlüsse als Initiierungsstellen gefunden werden, liegt an der Auslagerung des Materials. Es kann mit Sicherheit davon ausgegangen werden, dass längliche Einschlüsse, wenn sie im Material vorhanden sind, eine höhere Anfälligkeit für Rissbildung darstellen als runde. Dennoch können, wie diese Arbeit zeigt, auch runde Einschlüsse als Initiierungsstellen für Risse fungieren.

\section{\begin{tabular}{l|l} 
Einfluss des Reinheitsgrades der Proben & 6.3
\end{tabular}}

Zur weiteren Untersuchung des Einflusses der Einschlüsse auf den Rissbildungsprozess unter Wasserstoffbeladung wurde ein weniger reines Eisen verwendet, mit welchem die gleichen Experimente durchgeführt wurden. Es handelt sich um ARMCO-Eisen mit einer Zusammensetzung, die in Tabelle 4.2 gegeben ist. Die Korngröße des ARMCO-Eisens ist etwa halb so groß wie die des reinen Eisens und liegt damit in der gleichen Größenordnung. Die Blisterverteilung auf der Probenoberfläche und der Risse im Querschnitt der beiden verschieden Eisen-Proben nach Wasserstoffbeladung sind in den Abbildung 5.39 und 5.40 gegenübergestellt. Man erwartet, dass es im ARMCO-Eisen durch die geringere Reinheit mehr Einschlüsse gibt und daher auch mehr potentielle Initiierungsstellen. Im Vergleich der Blisterverteilung auf der Oberfläche bestätigt sich dies: Man beobachtet eine deutlich höhere Dichte von Blistern auf der Oberfläche. Daraus kann geschlossen werden, dass bei Wasserstoffbeladung schon direkt unter der Oberfläche bei ARMCO-Eisen mehr Risse initiiert werden, da die Dichte der Einschlüsse höher ist. Zunächst überraschend 
war die Beobachtung, dass sich bei ARMCO-Eisen die Risse nur direkt (maximal circa $100 \mu \mathrm{m}$ ) unter der Oberfläche befinden. Tief im Material liegt keine makroskopische Schädigung des Materials durch Risse vor. Die Verteilung der Einschlüsse und damit der potentiellen Initiierungsstellen sollte genauso homogen sein wie bei reinem Eisen, da eine identische Probenpräparation und Auslagerung durchgeführt wurde. Daher kann nur eine niedrigere Wasserstoffkonzentration dieses Phänomen erklären: Durch die erhöhte Konzentration an Einschlüssen in ARMCO-Eisen und der damit verbundenen größeren Anzahl an Rissen direkt unter der Oberfläche, kann der Wasserstoff nicht in genügend hoher Konzentration zur Rissinitiierung in größere Tiefe vordringen. Dadurch gibt es keine Risse in der Tiefe. Diese Vermutung wird durch Permeationsmessungen unterstützt. Für diese war es zunächst sinnvoll zu ermitteln, bei welcher Beladestromstärke Rissbildung einsetzt. Diese Messung wurde mittels Schmelzextraktionsmethode durchgeführt und ist in Abschnitt 5.1.8 zu finden. Hier wurde in einem Reihenversuch der Wasserstoffgehalt der Proben nach der Beladung bestimmt.

Die Permeationsmessungen sind in Abbildungen 5.48 und 5.47 dargestellt. Es handelt sich um eine Beladung, bei der die Beladestromstärke schrittweise erhöht und die Wasserstoffpermeation aufgezeichnet wird. Bei den ersten Beladestufen ist zu beobachten, dass die Permeation nach der Erhöhung der Beladestromstärke ansteigt und in eine Sättigung übergeht. Wird der Strom so weit erhöht, dass Rissbildung auftritt, wird ein verändertes Verhalten beobachtet. Die Permeation steigt an, durchläuft ein Maximum und fällt ab. Dieses kann wie folgt erklärt werden: Eine höhere Beladestromstärke führt zunächst zu einer höheren Wasserstoffpermeation durch die Probe. Ebenfalls können sich nach kurzer Zeit durch die höhere Wasserstoffkonzentration Risse bilden. In diesen wird Wasserstoff als Gas getrappt und die Permeation wird dadurch herabgesetzt. Finden sich nun wie bei ARMCO-Eisen direkt unter der Oberfläche viele Einschlüsse im Material, werden hier viele Risse initiiert und tiefer im Material ist die Wasserstoffkonzentration nicht hoch genug, um weitere Risse zu initiierten. Diese Erkenntnis, dass vorhandene Risse die Permeation reduzieren, erklärt auch die Beobachtung, dass bei reinem Eisen Risse tief im Material gebildet werden können. Denn bei reinem Eisen liegt eine geringere Dichte an Einschlüssen vor, sodass weniger Risse direkt unter der Oberfläche initiiert werden können. Ab einer bestimmten Tiefe wurden auch in diesem Material so viele Risse gebildet, dass die Konzentration in darunter liegendem Material nicht ausreicht, um weitere Risse zu bilden. In dieser Arbeit war die Dicke der Proben maximal 1,5 mm, sodass in der gesamten Tiefe der Probe Risse zu finden waren. In der Bachelorarbeit von Ferdinand Döring [Dö15] hingegen konnte an dickeren Proben gezeigt werden, dass die Risse nicht die gesamte Probendicke durchziehen.

Darüber hinaus wurde auch ein möglicher Einfluss der Rissinitiierung durch eine Veränderung der Korngröße untersucht. Eine etwa eine Größenordnung kleinere Korngröße konnte durch Kaltwalzen und eine anschließende moderate Auslagerung des reinen Eisen erreicht 
werden. Die Prozedur ist in Kapitel 4 näher beschrieben. Eine Gegenüberstellung von Blister- und Rissverteilung ist in den Abbildungen 5.39 und $5.41 \mathrm{zu}$ sehen. Man erkennt keine deutlichen Unterschiede. Die Blisterdichte auf der Oberfläche ist ähnlich, eventuell etwas erhöht gegenüber dem Eisen mit größeren Körnern. Dieses kann durch die geringere Probendicke oder die Länge der Risse erklärt werden, auf die später genauer eingegangen wird. Die Risse ziehen sich durch den gesamten Querschnitt der Probe, dieses entspricht der Erwartung, da sich die Verteilung der Einschlüsse gegenüber dem reinen und gut ausgelagerten Eisen nicht verändert haben sollte. Dadurch kann mit Sicherheit gesagt werden, dass die Initiierung der Risse an Einschlüssen von der Dichte und Verteilung der Einschlüsse im Eisen abhängt und nicht durch die Korngröße beeinflusst wird. Eine detaillierte Studie der chemischen Zusammensetzung der Einschlüsse in ARMCO-Eisen war experimentell deutlich schwieriger, denn aufgrund der Lage der Risse nur direkt unter der Oberfläche können sie nicht oder nur zu einem kleinen Teil durch einen Zugversuch zur Beobachtung freigelegt werden. Der Bruch findet nach einer starken Einschnürung durch die Bildung einer schmalen Kante statt, wie es für Eisen ohne Risse im Inneren typisch ist. Am Rand können nur einzelne Einschlüsse in Wasserstoff-induzierten Rissen identifiziert und vermessen werden. Die REM-Aufnahmen einer ARMCO-Eisen Probe und eine EDX-Analyse eines Einschlusses ist in den Abbildungen A.1 bis A.3 abgebildet. Bei diesen handelt es sich um Aluminiumoxid, wie es auch bei reinem Eisen nachgewiesen wurde.

\section{Bildung eines Spalts zwischen Einschlüssen und umgebender Eisenmatrix

Im vorherigen Abschnitt wird der experimentelle Fund beschrieben, dass Risse an Einschlüssen initiiert werden. Eine thermodynamische Begründung wurde im Theorieteil hergeleitet und ist in Abbildung 2.16 veranschaulicht. Hier ist skizziert, dass die Bildung von zwei neuen Oberflächen thermodynamisch günstiger sein kann als das Bestehen der Grenzfläche zwischen Matrix und Einschluss, wenn Wasserstoff an der Grenzfläche gelöst ist. So kann es zur Spaltung an der Grenzfläche zwischen Einschluss und umgebender Eisen-Matrix kommen. Voraussetzung ist ein hohes chemisches Potential, welches durch die elektrochemische Beladung erreicht wird, jedoch nicht quantifiziert werden kann. Eine detaillierte Beschreibung des Vorgangs ist in [KSS15] zu finden.

In REM-Aufnahmen der Einschlüsse, vergleiche zum Beispiel Abbildung 5.15, wurde festgestellt, dass zwischen Einschluss und Eisenmatrix immer ein Spalt ist. Die Breite des Spaltes beträgt bis zu $2 \mu \mathrm{m}$. Wenn eine Dekohäsion von Einschluss und Matrix stattfindet, wie es oben beschrieben wurde, würden die beiden neu gebildeten Oberflächen zunächst direkt aufeinander liegen. Die Entstehung des freien Volumens im Spalt kann dadurch nicht erklärt werden. Ein denkbarer Prozess, der zur Bildung des Spalts führen kann, ist eine Ansammlung von Leerstellen. Leerstellen und Leerstellencluster liegen, wenn 
Wasserstoff im Material gelöst ist, in erhöhter Konzentration vor [Fuk03]. Eine detaillierte theoretische Beschreibung der Wechselwirkung von Leerstellen und Wasserstoff in $\alpha$-Eisen wurde von Hayward und Fu [HF13] durchgeführt. Sie beschreiben, dass sowohl die Diffusion von Wasserstoff als auch von Leerstellen durch die starke Bindung der beiden generell herabgesetzt wird, wobei ein Komplex aus $\mathrm{H}_{m} \mathrm{~V}_{3}$ (V: Leerstelle) eine Ausnahme bildet und schnell diffundiert. Generell kann es zu einer Art Mittragen von Leerstellen durch die Wasserstoffdiffusion und umgekehrt kommen. Leerstellen-Wasserstoff-Cluster können an der Grenzfläche, beziehungsweise der neu gebildeten Oberfläche um den Einschluss, getrappt werden. Dadurch vergrößert sich der Spalt zwischen Einschluss und Matrix. Zusätzlich wird Wasserstoff zu dem Spalt transportiert. Der Wasserstoff rekombiniert zu $\mathrm{H}_{2}$ und es baut sich ein Wasserstoffdruck zwischen Matrix und Einschluss auf. Ein starkes Indiz für diesen Druck lieferte eine TEM-Untersuchung. Hier wurde das Material direkt unter einer Rissinitiierungsstelle untersucht, vergleiche Abbildung 5.36. Hier konnte beobachtet werden, dass sich das Material rund um das Loch konzentrisch verformt hat. Diese Verformung deutet darauf hin, dass innerhalb des Loches, genauer in dem Spalt zwischen Einschluss und Matrix, ein hoher Druck geherrscht haben muss. Die makroskopischen Risse, die innerhalb des Materials nach der Wasserstoffbeladung beobachtet werden, gehen von den Einschlüssen aus. Diese wurden also zunächst durch eine Dekohäsion von der Matrix gespalten, dann bildete sich durch Diffusion von Leerstellen-Wasserstoff-Komplexen ein Spalt zwischen Matrix und Einschluss. In diesem baute sich durch die Rekombination von Wasserstoff zu $\mathrm{H}_{2}$ ein hoher Gasdruck auf, dessen Verformung im umliegenden Material durch TEM Aufnahmen sichtbar gemacht werden konnte. Als Triebkraft für die Rissinitiierung als auch für das sich anschließende Risswachstum ist der Wasserstoffdruck zunächst in dem Spalt zwischen Matrix und Einschluss und später in den Rissen wahrscheinlich, wie es auch schon in der Literatur vorgeschlagen wurde, zum Beispiel in $\left[\mathrm{RZC}^{+} 07\right]$.

\section{Der Wasserstoffdruck als Triebkraft für die Rissbildung}

\section{5}

In der Literatur wird vielfach ein hoher Wasserstoffdruck als Triebkraft der Rissbildung vorgeschlagen [TR61, MS84, RZC $^{+} 07$ ]. Ein direkter Nachweis beziehungsweise eine Messung des Drucks innerhalb der Risse ist nur schwer durchführbar. Messungen des Drucks in einzelnen Rissen durch Neutronenstreuung wurden von Grische et al. [GDK $\left.{ }^{+} 14\right]$ durchgeführt. Es wurden Drücke von bis zu 20\% der Streckgrenze von ARMCO-Eisen gemessen. In der vorliegenden Arbeit kam eine komplementäre Messmethode zum Einsatz, denn es wurde der Wasserstoffdruck gemittelt über alle Risse im Material bestimmt. Es wurde über die Dichte der Probe nach der Wasserstoffbeladung das Gesamtvolumen aller Risse bestimmt. Anschließend wurde das Ausgasen der Proben in eine Vakuumkammer nach 
der Wasserstoffbeladung durch eine Druckmessung dokumentiert. Es konnte so die Menge an Wasserstoff bestimmt werden, die nach der Beladung in der Probe vorlag. Der Druck in den Rissen kann über die Menge an Wasserstoff und das Volumen der Risse mit der Van-der-Waals Gleichung berechnet werden. Die Dichtemessung wurde in der Bachelorarbeit von Lehmberg [Leh14] entwickelt. In ihrer Arbeit wurde der Wasserstoffaustritt über eine Messung der Probenmasse bestimmt, was in dieser Arbeit durch eine Druckmessung in einer Vakuumkammer ersetzt wurde, denn die Messung der Masse wurde durch Drift der Waage verfälscht. Zusätzlich kam es zu einer Oxidation der Probe und einem damit verbundenen Anstieg der Masse, der die Messung des Wasserstoffaustritts überlagerte. Die Messung des Drucks in der Vakuumkammer ist sehr genau und die einzig denkbare Fehlerquelle ist ein Leck der Kammer. Dieses minimale Leck wurde durch Stabilitätsmessungen erfasst und durch Verwendung einer Korrekturgeraden eliminiert. Der berechnete Fehler setzt sich aus den Abweichungen der Dichtemessungen untereinander und den Schwankungen während der Stabilitätsmessungen durch Fehlerfortpflanzung zusammen.

Es wird somit der Wasserstoffdruck in den Rissen nach der Beladung gemessen, wobei beachtet werden muss, dass die Überführung der Probe in die Vakuumkammer etwa 2 Minuten in Anspruch nimmt. Das Ausgasen der Probe ist, wie in Abbildung $5.46 \mathrm{zu}$ sehen, direkt nach der Beladung am schnellsten. Ein gewisser Anteil an Wasserstoff ist somit schon vor Beginn der Messung aus der Probe ausgetreten. Des Weiteren könnte eine geringe Menge an Wasserstoff in der Probe verbleiben. Bei reinem Eisen ist vermutlich noch etwas mehr Wasserstoff in der Probe, da das Ausgasen der in der Probenmitte liegenden Risse langsamer als bei ARMCO-Eisen war und dadurch die Ausgasrate am Ende der Messung bei reinem Eisen noch etwas höher ist als bei ARMCO-Eisen. Das bedeutet, dass der Druck in den Rissen bei der verwendeten Messmethode vor allem wegen der zeitlichen Verzögerung zwischen Beenden der elektrochemischen Wasserstoffbeladung und Beginn der Druckmessung unterschätzt wird. Es wird also eine untere Grenze für den Wasserstoffdruck in den Rissen gemessen.

Der Druck beträgt bei beiden Eisensorten etwas über $50 \mathrm{MPa}$, wobei er bei ARMCOEisen etwas niedriger ist. Dies kann durch die Lage der Risse erklärt werden. In ARMCO Eisen sind die Risse nur unter der Probenoberfläche zu finden, dadurch kann das Material leichter verschoben werden als bei tiefer im Material liegenden Rissen. Daher wird auch der Druck niedriger sein, der zu einem Rissfortschritt führt.

Die Streckgrenze der verwendeten Eisenproben wurde gemessen und liegt bei circa 65$75 \mathrm{MPa}$, vergleiche Abschnitt 5.1.11. Damit liegen die gemessenen Wasserstoffdrücke in den Rissen bei 70 bis $80 \%$ der Streckgrenze. Griesche et al. [GDK $\left.{ }^{+} 14\right]$ folgerten aus Messungen mit Neutronenstreuung einen Wasserstoffdruck von 20\% der Streckgrenze des dort verwendeten Eisens. Die Druckmessungen in der vorliegenden Arbeit konnten schneller nach der Beladung gestartet werden als in der Arbeit von Griesche et al. [GDK $\left.{ }^{+} 14\right]$. In deren Arbeit konnte die Messung erst 14 Stunden nach der Beladung gestartet werden. In 
Abbildung 5.46 ist der Druck in den Rissen in Abhängigkeit der Zeit nach der Beladung gezeigt. Nach 14 Stunden ist der Druck in den Rissen schon auf circa die Hälfte des Anfangsdruckes abgefallen. Dadurch kann man von einer guten Übereinstimmung mit den Messungen von Griesche et al. [GDK $\left.{ }^{+} 14\right]$ sprechen.

Eine obere Abschätzung des Wasserstoffdrucks in den Rissen wurde von Kirchheim in $\left[\mathrm{TML}^{+} 16\right]$ bestimmt. Hierbei wurde über eine Beladung mit einem stärkeren Wasserstoffpromoter und den darüber maximal in der Probe gelösten Wasserstoff der Druck berechnet. Er beträgt 1,8 GPa für das verwendete reine Eisen. Damit kann ein Bereich möglicher Wasserstoffdrücke in Rissen und Hohlräumen in Eisen angegeben werden:

$$
p_{\text {Risse }}=50-1800 \mathrm{MPa} \text {. }
$$

Der angegebene Bereich umfasst eine große Druckspanne. Das liegt zum einen daran, dass die untere Grenze, wie oben beschrieben, unterschätzt und die obere Grenze ein wenig überschätzt wird, denn ein Teil des gebildeten Wasserstoffs wird nicht in der Probe gelöst, sondern rekombiniert auf der Probenoberfläche zu $\mathrm{H}_{2}$. Allerdings ist zu erwarten, dass für die erste Rissinitiierung, ein deutlich höherer Druck im Spalt zwischen Einschluss und Matrix herrschen muss, damit der Riss initiiert wird, als für den späteren Rissfortschritt, denn es wird ein niedrigerer Druck benötigt, wenn der Riss länger ist und wenn die Rissspitze einen kleineren Krümmungsradius hat. In Abschnitt 5.2 ist die Finite-Elemente Simulation eines elliptisch geformten Risses in linear-elastischem Eisen gezeigt. Es ergibt sich für einen Riss der Länge $100 \mu \mathrm{m}$ mit einem Krümmungsradius von 0,25 $\mu \mathrm{m}$ eine Spannung im Material etwa $2 \mathrm{GPa}$. Bei einem runden Loch ist keine signifikante Spannungskonzentration zu erwarten. Um die gleiche Spannung im Material zu induzieren, muss also ein Druck von circa $2 \mathrm{GPa}$ im runden Loch herrschen. Der Wert von $2 \mathrm{GPa}$ entspricht in etwa der oberen Grenze für den Druck, der aus der Permeationsmessung ermittelt wurde. Somit erscheint der angegebene Druckbereich realistisch, da die Rissinitiierung von einem runden Loch aus einen wesentlich höheren Druck erfordert als ein Rissfortschritt eines bereits langen Risses. Ein langer Riss hat eine Rissspitze, vor der eine Spannungskonzentration im Material induziert wird. Zusammenfassend stellt der Druck von 1,8 GPa den notwendigen Druck dar, um Risse zu initiieren, und der gemessene Druck von $50 \mathrm{MPa}$ stellt die untere Grenze für den Druck dar, der nötig ist um Risse zu vergrößern.

Die Kinetik des Ausgasens konnte durch die Druckmessung in der Vakuumkammer sichtbar gemacht werden, siehe Abbildung 5.45. Das Ausgasen der Probe dauert etwa 100 bis 300 Stunden. Der Diffusionskoeffizient von Wasserstoff in Eisen liegt in der Größenordnung von $10^{-8} \mathrm{~m}^{2} / \mathrm{s}$ [GR00]. Der Wasserstoff kann demnach in wenigen Minuten durch eine $1,5 \mathrm{~mm}$ dicke Probe diffundieren. Daher ist es unwahrscheinlich, dass die Kinetik wesentlich von der Bulkdiffusion abhängt. Vielmehr muss der Wasserstoff, der molekular in den Rissen vorliegt, zunächst dissoziieren, damit er vom Eisen in atomarer Form aufgenommen werden kann. Thermodynamisch kann die Lösung von Wasserstoff in Eisen 
durch ein Streben zum Gleichgewicht nach dem Sievertsschen Gesetz verstanden werden. Im Fall von ARMCO-Eisen diffundiert er dann zur Oberfläche und kann hier unter Bildung von Wasserstoffmolekülen entweichen. Im Falle von reinem Eisen kann Wasserstoff, der an einem tief im Material liegenden Riss dissoziiert wird, auf seinem Weg zur Probenoberfläche auf andere Risse treffen und in diesen wiederum als Gas getrappt werden. Dies erklärt das deutlich schnellere Ausgasen von ARMCO-Eisen, das in Abbildung 5.45 $\mathrm{zu}$ beobachten ist.

\section{Diskontinuierlicher Rissfortschritt durch das Material

Nachdem der Druck im Spalt zwischen Matrix und Einschluss und später in den Rissen als Triebkraft für die Rissbildung ausgemacht wurde, stellt sich die Frage, wie die Rissausbreitung durch das Material verläuft. Zunächst wird in diesem Abschnitt diskutiert, ob es sich um ein schlagartiges Entstehen der Risse, um ein langsames kontinuierliches oder ein diskontinuierliches Risswachstum handelt. Dafür wurden von den Proben im Elektrolyten während der Wasserstoffbeladung Bilder mit einer Mikroskopkamera aufgenommen. Somit konnte das Wachstum einzelner Blister auf der Oberfläche beobachtet werden. Eine Serienaufnahme eines Wachstumsprozesses ist in Abbildung 5.24 zu sehen. In der gezeigten und in weiteren Messungen ist zu sehen, dass ein Blister innerhalb einiger Minuten auf seine endgültige Größe heranwächst. Ein schnelles Risswachstum (von Sekunden oder Sekundenbruchteilen) des gesamten Risses kann aufgrund der Serienaufnahmen ausgeschlossen werden.

Zusätzlich zu den Serienaufnahmen erlaubt das äußere Erscheinungsbild eines Blisters, Rückschlüsse auf das Wachstum zu schließen. Eine REM-Aufnahme eines typischen Blister ist in Abbildung 5.6 gezeigt. Der Blister besteht aus einem Plateau, welches von Stufen auf den Flanken umgeben ist. Die Stufen können mit einzelnen Rissfortschritten in Zusammenhang gebracht werden und somit liegt dann ein diskontinuierliches Wachstum des Blisters vor. Die Form wurde durch eine Profilmessung bestätigt, vergleiche Abbildung 5.4. Das Auftreten eines Plateaus wurde bisher in der Literatur nicht berichtet, wobei es nur wenige Autoren gibt, die die Form der Blister näher betrachtet haben. Escobar et al. $\left[\mathrm{EMD}^{+} 11\right]$ führten eine detaillierte Studie durch, unter welchen Bedingungen es zur Blisterbildung kommt, und betrachteten Blister im REM. Allerdings verwendeten diese Autoren kaltgewalztes Material, welches sich im Bezug auf die Verformung des Materials deutlich anders verhält als ausgelagertes und dadurch defektarmes Material. Man kann in der Serienaufnahme in Abbildung 5.24 erahnen, dass sich das im REM beobachtete Plateau zuerst aus der Probenoberfläche erhebt und dann an den Flanken langsam mehr und mehr Stufen hinzu kommen. Diese Beobachtung bestätigt ein diskontinuierliches Wachstum des Blisters. 
Die Auswertung der Anzahl der Stufen des Blisters, welcher in der Serienaufnahme betrachtet wurde, ergibt eine Entstehungszeit von circa 13 s pro Stufe. Dabei wird von einer Bildung der Stufen im gleichen zeitlichen Abstand ausgegangen. Das bedeutet, etwa alle $13 \mathrm{~s}$ ist der Wasserstoffdruck in den Rissen, der als Triebkraft für den Rissfortschritt identifiziert wurde, so hoch, dass es zu einer Vergrößerung des Risses kommt. Dadurch wächst auch der Blister auf der Oberfläche. Bestätigt wird diese Vermutung eines „Stop and Go“Wachstums durch das Erscheinungsbild der Rissflächen, welche in REM-Aufnahmen der Bruchflächen zu sehen sind, siehe Abbildung 5.16. In dieser exemplarischen Abbildung sieht man ein Loch (in dem sich ein Einschluss befand), an dem der Riss initiiert wurde, und konzentrisch darum sind Stufen angeordnet. Es ist naheliegend, dass sich bei jedem Rissfortschritt eine Stufe gebildet hat. Durch dieses diskontinuierliche Wachstum kommt es zu einer starken Rissöffnung und einem damit verbundenen großen Volumen des Risses. Dieses konnte in Querschnittsaufnahmen beobachtet werden, vergleiche Abbildung 5.12. Hier ist zu sehen, dass sich der Riss circa $30 \mu \mathrm{m}$ geöffnet hat.

\section{\begin{tabular}{l|l} 
Rissfortschritt durch Quasispaltbruch & 6.7
\end{tabular}}

Neben den eben diskutierten Stufen kann man in der Aufnahme der Rissfläche, siehe Abbildung 5.16, Streifen auf den Stufen erkennen. Diese Streifen verlaufen in Rissausbreitungsrichtung. Sie werden oft bei Wasserstoff-induzierten Brüchen beobachtet und sind detailliert in der Arbeit von Martin et al. $\left[\mathrm{MFL}^{+} 11\right]$ diskutiert worden. Man bezeichnet diese Art der Bruchflächen als Quasispaltbruch. In Detailaufnahmen der Bruchflächen, vergleiche Abbildung 5.17, kann man erkennen, dass es sich bei den Streifen um leichte Erhebungen aus der Bruchfläche handelt. Sie sind sehr dünn und dadurch im REM bei $30 \mathrm{kV}$ teilweise elektronentransparent. Diese Form lässt auf eine lokalisierte duktile Verformung schließen, denn die Entstehung der Streifen ist ähnlich zu verstehen wie die Entstehung einer sogenannten „Messerkante“ bei dem makroskopisch duktilen Bruch einer Probe. Sie wurde in der Theorie in Abbildung 2.8 eingeführt. Das bedeutet, dass ein duktiler Mechanismus bei der Bildung der Streifen zu Grunde liegen muss. Martin et al. $\left[\mathrm{MFL}^{+} 11\right]$ wiesen nach, dass in ähnlichen Materialien unterhalb solcher Streifen eine duktile Verformung in Form von hohen Versetzungsdichten und Gleitbändern vorliegt. Durch Zugexperimente konnte gezeigt werden, dass makroskopisch eine Versprödung des Materials durch den gelösten Wasserstoff vorliegt. Die Zugexperimente wurden von Jonas Wawra im Rahmen seiner Bachelorarbeit mit zu den in dieser Arbeit identischen Proben durchgeführt [Waw16], vergleiche Abbildungen 5.49 und A.4. Es ist zu erkennen, dass die Bruchdehnung von circa $60 \%$ auf etwa $15 \%$ zurückgeht. Dabei muss beachtet werden, dass auch durch die Risse in der Probe eine Verringerung der Bruchdehnung verursacht wird. Dieses Verhalten wurde ebenfalls in der Arbeit von Wawra [Waw16] untersucht. Durch 
einen Zugversuch an einer beladenen und dann entgasten Probe konnte gezeigt werden, dass die Bruchdehnung einer Probe mit Rissen aber ohne gelösten Wasserstoff bei circa $30 \%$ liegt. Es kann daher geschlossen werden, dass, wie für Eisen erwartet, eine Versprödung des Materials durch den gelösten Wasserstoff auftritt. Der Wasserstoff ist also nicht nur Triebkraft der Rissbildung durch den hohen Druck in den Rissen, sondern beeinflusst auch das mechanische Verhalten.

Da der generelle Bruchprozess eher spröde ist, beobachtet man glatte Flächen auf den Stufen; eine Ausnahme bilden die Streifen, welche sehr dünn sind und somit einen duktilen Charakter haben.

\section{\begin{tabular}{l|l} 
Mikrostruktur & 6.8
\end{tabular}}

In dieser Arbeit wurden TEM-Lamellen aus Bruchflächen sowie aus der Probenoberfläche und der Querschliffläche extrahiert, um den Charakter des Rissfortschritts und die zugrunde liegende Defektstruktur besser zu verstehen. Der Charakter wurde schon in der Diskussion der Rissflächen im vorherigen Abschnitt behandelt, es wurden lokal duktile Elemente auf generell spröde aussehenden Rissflächen nachgewiesen.

Zunächst wurde das Material zwischen den Blistern untersucht, um festzustellen, ob es eine langreichweitige plastische Verformung gibt. Diese ist nicht vorhanden, da in einer TEM-Lamelle, die von der Probenoberfläche aus präpariert wurde, einkristallines nahezu defektfreies Material zwischen den Blistern beobachtet wurde, vergleiche Abbildung 5.29. Das Material weist keine erhöhte Defektdichte im Vergleich zu vor der Beladung auf. Eine TEM-Untersuchung der Defektstruktur parallel zu einem Riss, bei welcher die TEMLamelle einer Entfernung von einigen Mikrometern extrahiert wurde, zeigt eine vergleichsweise sehr hohe Versetzungsdichte. Dieses ist vor allem an der Bildung von Zellwänden durch die Ansammlung von Versetzungen zu erkennen, vergleiche Abbildung 5.35. Durch diese Untersuchung lässt sich bestätigen, dass es auf lokaler Skala in der Größenordnung von einigen Mikrometern Merkmale von duktiler Verformung gibt.

Weitere TEM-Untersuchungen, zum Beispiel des Querschnitts der Flanke eines Blisters, vergleiche Abbildung 5.32, und des Materials unterhalb einer Rissinitiierungsstelle, siehe Abbildung 5.36, zeigen ebenfalls Merkmale duktiler Verformung. Man kann eine erhöhte Versetzungsdichte gegenüber dem unbeladenen Material und vor allem Scherbänder beobachten. Diese Scherbänder unterhalb der Stufen eines Blister konnten, wie in Abschnitt 6.6 diskutiert wurde, mit einem Rissfortschritt in Verbindung gebracht werden. Bei einem Rissfortschritt wird jeweils das Volumen des Risses vergrößert und Material zur Oberfläche verschoben. Wie aus der TEM-Aufnahme Abbildung 5.32 hervorgeht, wird bei dieser Materialverschiebung ein Scherband im Material erzeugt. Es reicht von einer Stufe, die auf der Rissfläche beobachtet wird, bis zu einer Stufe auf einem Blister. Die 
Scherbänder, die zu den verschiedenen Stufen auf einem Blister gehören, sind im Material darunter parallel angeordnet, vergleiche Abbildung 5.32.

Bei dem verwendeten Eisen sind die Körner so groß, dass sich ein Riss maximal über einige Körner erstreckt, oft ist er sogar nur innerhalb eines Korns. Somit handelt es sich um eine vom Druck in den Rissen hervorgerufene Einkristallverformung. Allerdings ist Wasserstoff im Material gelöst, dieses führt zu einer Veränderung im Verhalten während der Verformung. So wurden im Theorieteil verschiedene mögliche Mechanismen vorgestellt, die eine Veränderung des mechanischen Verhaltens erklären. Es kann hier festgestellt werden, dass eine sehr lokalisierte duktile Verformung vorliegt. Es kann allerdings kein Vergleichsexperiment durchgeführt werden, in welchem ein hoher Wasserstoffdruck in einem Riss vorliegt, aber kein Wasserstoff im Bulk gelöst ist. Es liegt nahe, dass eine Erleichterung von Versetzungsbildung und Versetzungsbewegung durch den Wasserstoff, wie es der HELP-Mechanismus beschreibt [Bea72], vorliegt. Dadurch kann es zu einer starken Lokalisation der duktilen Verformung kommen.

Mit Hilfe von Abbildung 5.32 konnte zusätzlich gefolgert werden, dass die Stufen des Risses, die im unteren Teil des Bildes zu sehen sind, entlang einer Gleitebene verlaufen. Dieses wurde detailliert mittels EBSD untersucht. Bei dieser Bestimmung muss beachtet werden, dass man den dreidimensionalen Verlauf des Risses nicht kennt, sondern nur seine Projektion in die Querschliffebene mit ausgewählten Ebenen verglichen werden kann. Es wurde jeweils der Winkel zur $\{100\}$ Spaltebene in Eisen und zu den verschiedenen Gleitebenen in Eisen verglichen. Man erkennt in einer Auftragung der Winkel zu den entsprechenden Ebenen, vergleiche Abbildung 5.23, dass eine Rissöffnung entlang Spaltebenen ausgeschlossen werden kann und eine der Gleitebenen jeweils die Rissebene darstellt, wobei bei einigen Rissen auch mehrere Gleitebenen in Frage kommen. Die Rissöffnung von Wasserstoffinduzierten Brüchen entlang von Gleitebenen wurde ebenfalls von [Ber70, NB78] in Zugexperimenten an einkristallinem Eisen nachgewiesen.

Bei einem spröden Bruch, wie er bei tiefen Temperaturen zu erwarten wäre, würde man einen Bruch entlang der Spaltebene erwarten [Pet58, Ber70]. Diese Art des spröden Bruches tritt nicht auf. Als Grund für den Bruch entlang von Gleitebenen kann man eine Schwächung der Bindungen zwischen den Gleitebenen annehmen. In der Literatur wird als alternativer Grund eine Verhinderung von Quergleiten angegeben [Pet58, Ber70].

Weitere TEM-Untersuchungen zeigen das Material unterhalb der Bruchflächen der Wasserstoff-induzierten Brüche. Sie bestätigen, dass bei Rissfortschritt ein Scherband erzeugt wird, vergleiche Abbildung 5.37. Weiter konnte eine TEM-Lamelle senkrecht zum Rissfortschritt präpariert werden, siehe Abbildung 5.38. In dieser ist die Defektstruktur unterhalb eines der Streifen, die auf den Stufen verlaufen zu sehen. Hier ist lediglich eine hohe Versetzungsdichte, aber keine Scherbänder zu beobachten. Das bedeutet, dass die Streifen durch eine lokale duktile Verformung zustande kommen und bei einem Bruch die zuletzt gerissenen Stellen sind. 
Es wurde eine TEM-Lamelle aus einer Bruchfläche präpariert, die das Material unterhalb einer Riss-Initiierungsstelle zeigt, vergleiche Abbildung 5.36. In der Abbildung ist das Loch markiert, in dem ursprünglich ein Einschluss war. Man erkennt ausgehend von diesem Loch eine konzentrische plastische Verformung, diese wurde schon in Abschnitt 6.4 diskutiert. Es handelt sich um Verformung, die durch den hohen Wasserstoffdruck verursacht wird, der sich vor der Riss-Initiierung zwischen Einschluss und Eisenmatrix aufbaut. Weiter lässt sich in der TEM-Abbildung beobachten, dass Scherbänder vorhanden sind, welche innerhalb des Materials etwa unterhalb des Loches enden. Sie sind mit Pfeilen in Abbildung 5.36 markiert. Bei der Untersuchung des Querschnitts eines Blisters wurden ebenfalls Scherbänder beobachtet, die mit einem Rissfortschritt in Verbindung gebracht werden konnten. Da die beobachteten Scherbänder nah an der Rissinitiierungsstelle liegen, muss es sich hierbei um die Verformung während der ersten Rissfortschritte handeln. Die Form eines Blisters, welche schon in Abschnitt 6.6 diskutiert wurde, kann durch diese TEM-Untersuchung nun näher erklärt werden. Die ersten Rissfortschritte erzeugen Scherbänder, die die Materialoberfläche nicht erreichen. Bei weiterer Vergrößerung des Risses, zumindest, wenn dieser nahe der Oberfläche liegt, erreichen die Scherbänder die Oberfläche und auf der Oberfläche werden die typischen Stufen eines Blisters erzeugt. Unterhalb des Plateaus des Blister befinden sich somit Scherbänder, die die Oberfläche nicht erreichen. Dies erklärt auch, warum oft verstärkt Lochfraß durch den sauren Elektrolyten auf dem Plateau der Blister beobachtet wurde, denn Lochfraß wird durch Versetzungen begünstigt [SI71]. 


\section{\begin{tabular}{l|l} 
& \\
Zusammenfassung & 7
\end{tabular}}

In diesem Kapitel sollen die Ergebnisse dieser Arbeit in Form eines Mechanismus der Rissbildung durch Wasserstoffbeladung in reinem Eisen zusammengetragen werden.
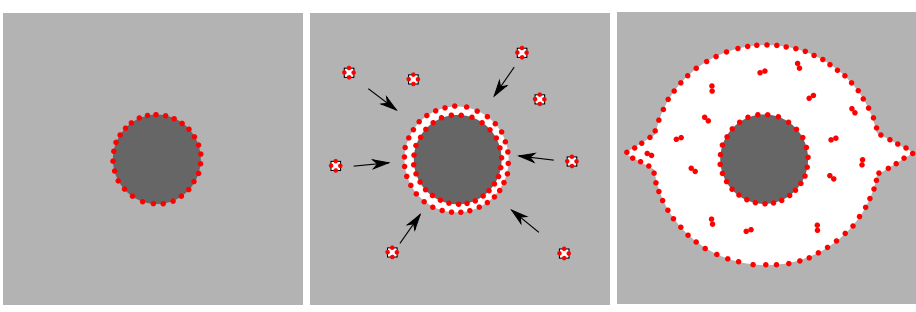

Abbildung 7.1: Schematische Darstellung des Mechanismus der Rissinitiierung an einem Einschluss im Material.

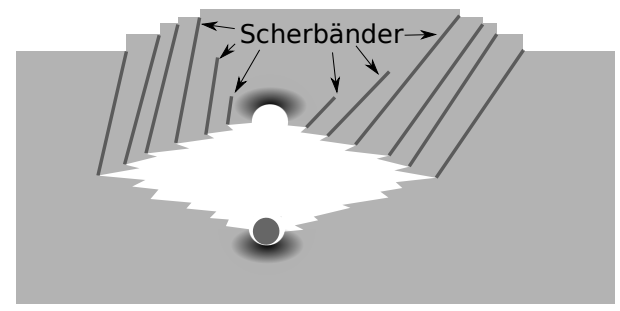

Abbildung 7.2: Schematische Darstellung der Defektstruktur zwischen einem Riss und einem Blister auf der Oberfläche.

In der vorliegenden Arbeit erfolgte eine elektrochemische Wasserstoffbeladung bei konstantem Strom in einem sauren Elektrolyten. Es wurde Eisen mit zwei verschiedenen Reinheitsgraden verwendet. Nach der Wasserstoffbeladung ist das Material von Rissen durchzogen und auf der Oberfläche befinden sich Blister über oberflächennahen Rissen. Für die Rissbildung konnte folgender Mechanismus erarbeitet werden:

- Wasserstoff wird durch die elektrochemische Beladung in der Probe gelöst. Er wird an Fehlstellen im Gitter, besonders an nichtmetallischen Einschlüssen, getrappt. Durch eine Freilegung der Bruchflächen durch Zugexperimente wurde festgestellt, dass es sich um runde oxidische Einschlüsse handelt.

- Es kommt zu einer Spaltung der Einschlüsse von der umgebenden Eisenmatrix. Dieser Vorgang kann thermodynamisch mit dem Defactants-Konzept erklärt werden. Es bildet sich ein Spalt zwischen Matrix und Einschluss, der in REM-Aufnahmen beobachtet wurde. Als Bildungsmechanismus des Spalts kommt eine Leerstellenansammlung in Frage, für diese konnte allerdings kein direkter Beweis erbracht werden. Schematisch ist der Vorgang der Bildung eines solchen Spaltes in Abbildung 7.1 zu sehen. 
- Im Spalt zwischen Einschluss und Matrix rekombinieren Wasserstoffatome zu Molekülen. Dadurch entsteht ein hoher Druck, welcher indirekt durch radiale plastische Verformung um das Loch eines Einschlusses in TEM-Untersuchungen beobachtet werden konnte, vergleiche Abbildung 7.2.

- Weiter konnte per Druckmessung in den Rissen ein hoher Wasserstoffdruck gemessen werden. Der gemessene Druck liegt bei: $p_{\text {Risse }} \geqq 50 \mathrm{MPa}$. Die Streckgrenze des verwendeten Materials liegt bei 65-75 MPa. Damit konnte der Wasserstoffdruck als Triebkraft für die Rissbildung identifiziert werden.

- Das Wachstum der Risse verläuft diskontinuierlich. Das bedeutet, dass der Druck im Riss bis zu einem kritischen Wert steigt, bei dem es zu einem Rissfortschritt kommt. Durch das gestiegene Volumen des Risses wird der Druck gesenkt. Über den weiterlaufenden Antransport von Wasserstoff steigt der Druck wieder bis zum nächsten Rissfortschritt an.

- Eine Untersuchung der Defektstruktur um die Risse mittels TEM ergab, dass lokal eine hohe plastische Verformung vorliegt, die mit dem HELP Mechanismus im Einklang ist. Die Materialverschiebung hin zur Oberfläche, die zur Blisterbildung auf der Oberfläche führt, ist mit der Bildung von Scherbändern verbunden. Die Bildung eines Scherbands wird bei einem Rissfortschritt hervorgerufen. Die Scherbänder enden an der Oberfläche mit einer Stufe, siehe Abbildung 7.2. Die Stufen bilden die Flanken eines Blisters.

Fazit: In der vorliegenden Dissertation konnte gezeigt werden, dass Rissbildung durch Wasserstoff ohne äußere mechanische Belastung nicht durch die Wahl eines sehr reinen Materials verhindert werden konnte. Es kommt zu Rissinitiierungen an Einschlüssen. Daraus kann geschlossen werden: Sofern einzelne Einschlüsse in einem Werkstoff vorhanden sind, können diese unter Wasserstoffbeladung den Ausgangspunkt für ein Versagen eines Bauteils sein. Eine Agglomeration von Leerstellen konnte nicht nachgewiesen werden und daher wird dieser Prozess als Initiierungsmechanismus generell als sehr unwahrscheinlich eingestuft. Eine Reduzierung des Wasserstoffgehaltes durch eine hohe Rissdichte unter der Oberfläche führte in ARMCO-Eisen dazu, dass keine Risse tief im Inneren des Materials entstehen konnten. Diese Beobachtung zeigt, dass eine Reduzierung von Einschlüssen einen negativen Effekt auf die Stabilität eines Bauteils haben kann.

Über die Bildung eines hohen Wasserstoffdruckes an Einschlüssen und später in Rissen hinaus, führt der gelöste Wasserstoff in Eisen zu einer Versprödung des Materials. Die duktilen Merkmale, die im Zusammenhang mit der Riss- und Blisterbildung beobachtet werden konnten, sprechen für eine lokalisierte plastische Verformung. Diese Beobachtungen sind auch auf Prozesse, in denen äußere mechanische Belastung auftritt, übertragbar und leisten einen Beitrag zum besseren Verständnis von Wasserstoffversprödung. 
Anhang 



\section{Zusätzliche Messungen}

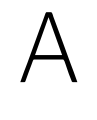

\section{Bruchfläche von ARMCO-Eisen}

In Abschnitt 5.1.2 ist beschrieben, dass mit Proben aus reinem Eisen Zugversuche bis zum Bruch der Probe zum Freilegen der Rissflächen durchgeführt wurden. Der gleiche Versuch wurde auch mit ARMCO-Eisen durchgeführt und die Ergebnisse sind in diesem Abschnitt dargestellt. Die Bruchfläche nach dem Zugversuch einer beladenen ARMCO-Eisenprobe

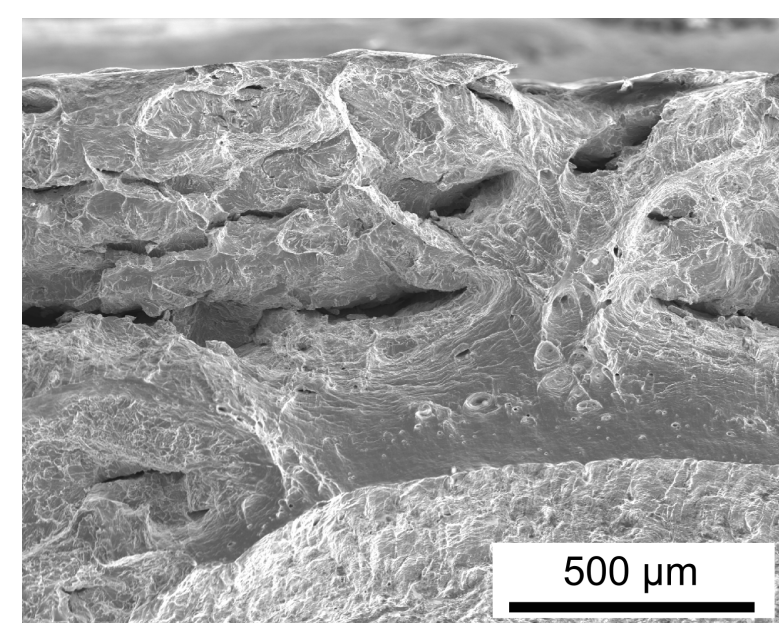

Abbildung A.1: REM-Aufnahme der Bruchfläche der ARMCO-Eisenprobe. Im unteren Teil des Bildes sieht man einen duktilen Bruch.

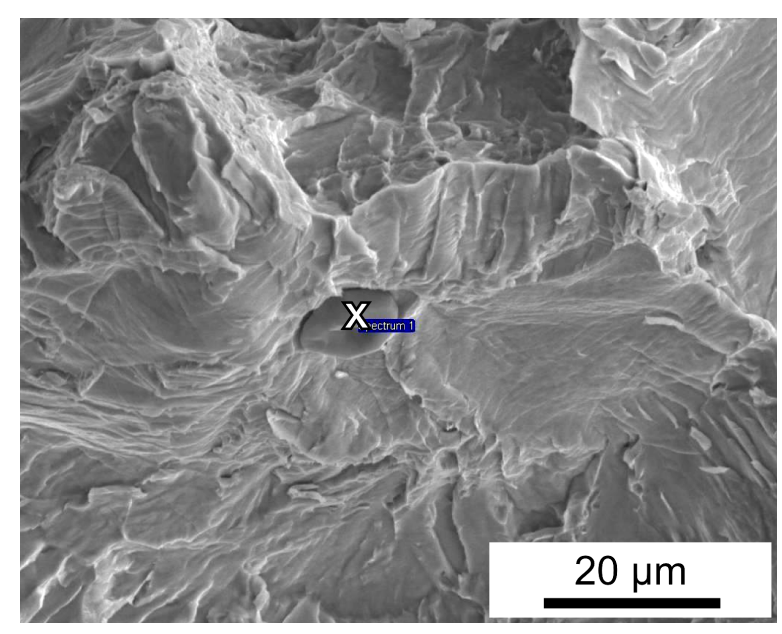

Abbildung A.2: REM-Aufnahme eines Einschlusses, der auf der Bruchfläche der ARMCO-Eisenprobe zu beobachten war. Am markierten Punkt wurde ein EDX-Punktscan durchgeführt (siehe Abbildung A.3).

ist in Abbildung A.1 zu sehen. Es ist im unteren Teil des Bildes ein duktiler Bruch zu erkennen, da sich eine sogenannte Messerkante gebildet hat, vergleiche Abbildung 2.8. Daraus kann geschlossen werden, dass in diesem Bereich der Probe keine Risse im Material vorlagen. Es ist ein duktiler Bruch der Probe zu erwarten. Im oberen Teil des Bildes erkennt man, dass Risse im Material vorlagen, welche bis zum Bruch angewachsen sind. Diese Risse lagen höchstwahrscheinlich parallel zur Oberfläche. Daher war nur bei wenigen Rissen ein Blick auf die Rissfläche möglich. Ein Beispiel ist in Abbildung A.2 gezeigt. In diesem Bild ist ein Einschluss zu sehen, der von Quasi-Spaltbruch umgeben ist. Die Rissfläche ähnelt der von reinem Eisen, vergleiche Abschnitt 5.1.2. Eine EDX-Analyse 


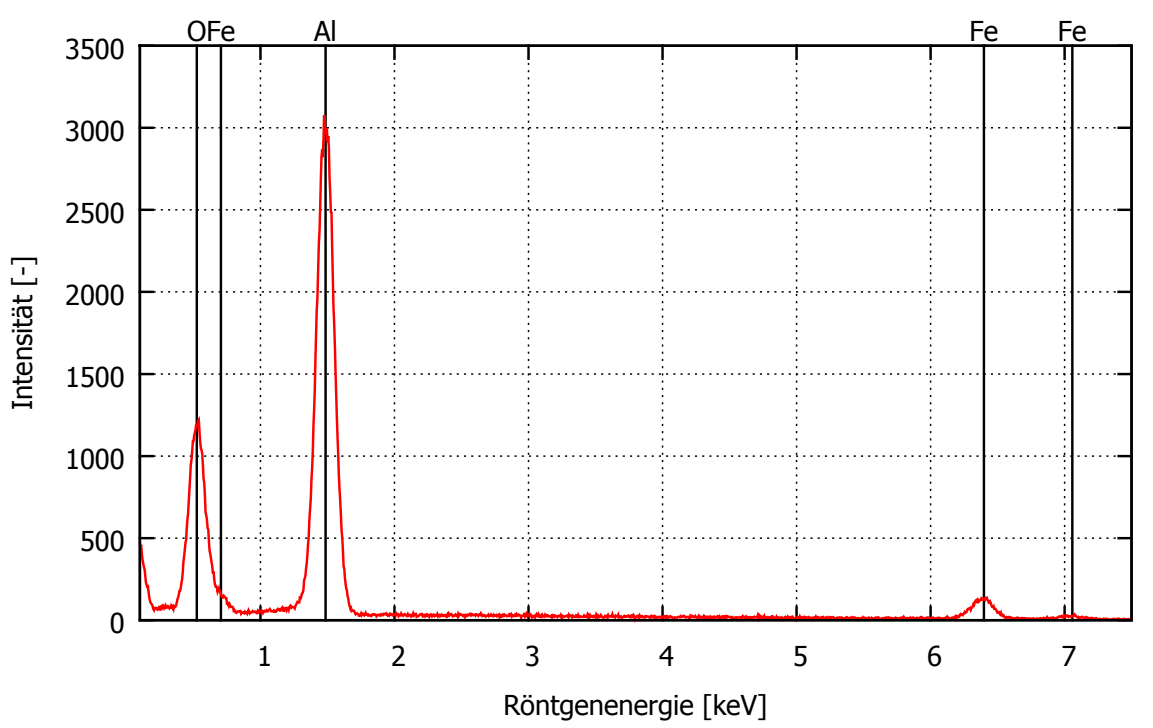

Abbildung A.3: EDX-Punktscan, der am Ort, der in Abbildung A.2 markiert ist, durchgeführt wurde. Man erkennt neben einem schwachem Eisen-Signal, nur Aluminium und Sauerstoff. Der vermessene Einschluss ist demnach Aluminiumoxid.

des Einschlusses ist in Abbildung A.3 gezeigt. Es handelt sich bei dem Einschluss um Aluminiumoxid. Dieses Oxid wurde ebenfalls in Messungen an Einschlüssen in reinem Eisen nachgewiesen. Zusammenfassend lässt sich feststellen, dass die Bruchfläche von ARMCO-Eisen aufgrund der Lage der Risse deutlich von der des reinen Eisen abweicht. Dadurch lassen sich die Rissflächen bei ARMCO-Eisen schlechter durch einen Zugversuch freilegen als bei reinem Eisen. 


\section{Zugversuch von ARMCO-Eisen}

Das Spannungs-Dehnungs-Diagramm, welches bei dem Bruch einer ARMCO-Eisenprobe aufgezeichnet wurde, ist in Abbildung A.4 dargestellt. Mit dieser Messung konnten die mechanischen Kennwerte Streckgrenze, Elastizitätsgrenze und Zugfestigkeit ermittlet werden. Sie sind Tabelle $5.2 \mathrm{zu}$ entnehmen. Ebenso ist festzustellen, dass die Bruchdehung ohne Beladung bei circa 60\% liegt und durch die Wasserstoffbeladung auf ungefähr 15\% zurückgeht. Es liegt also eine deutliche Versprödung des Materials vor.

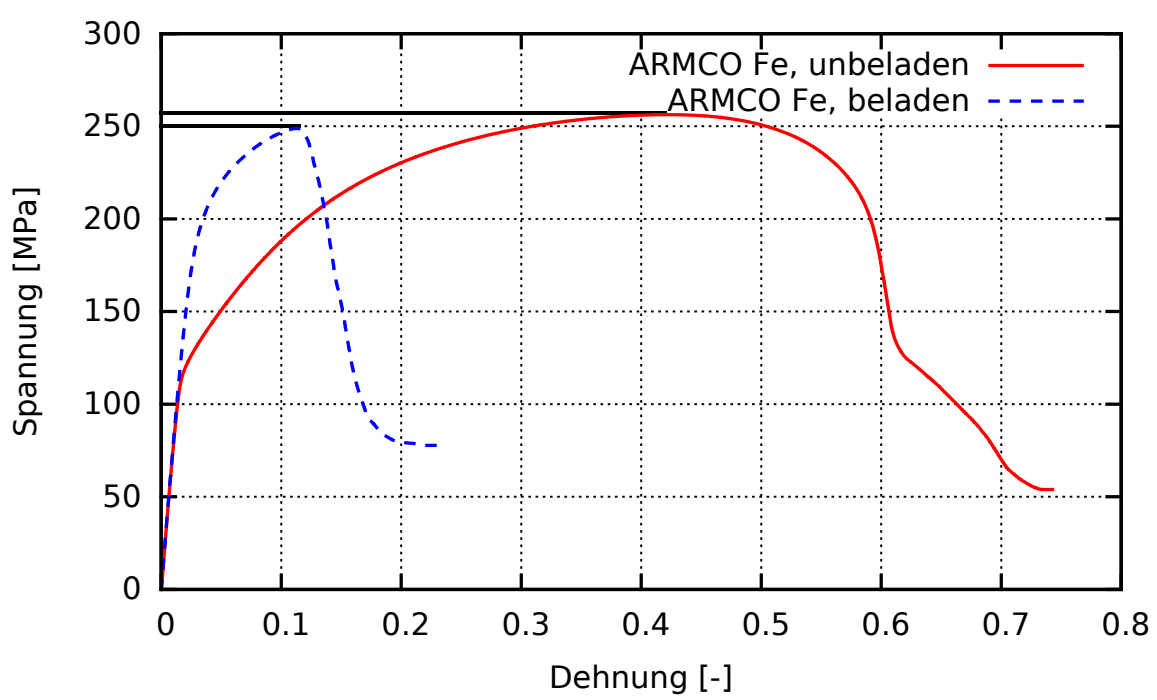

Abbildung A.4: Zugversuch von ARMCO-Eisen vor und nach Wasserstoffbeladung. Die schwarzen Linien kennzeichnen die gemessene Zugfestigkeit. Messung durchgeführt von Jonas Wawra [Waw16] 


\section{Liste der Veröffentlichungen}

\section{Veröffentlichungen}

$\left[\mathrm{TML}^{+}\right.$16] M. C. Tiegel, M. L. Martin, A. K. Lehmberg, M. Deutges, C. Borchers, and R. Kirchheim. Crack and blister initiation and growth in purified iron due to hydrogen loading. Acta Materialia 115, 24 (2016).

[BGT $\left.{ }^{+} 15\right]$ C. Borchers, C. Garve, M. Tiegel, M. Deutges, A. Herz, K. Edalati, R. Pippan, Z. Horita, R. Kirchheim. Nanocrystalline steel obtained by mechanical alloying of iron and graphite subsequently compacted by high-pressure torsion. Acta Materialia 97, 207 (2015).

\section{Konferenzbeiträge}

- M. C. Trynogga, A. Herz, Y. Chen, C. Borchers, R. Kirchheim. Stabilization of nanocrystalline iron by segregation of carbon at the grain boundaries. DPG-Frühahrstagung, Regensburg (2013).

- M. Trynogga, C. Borchers, R. Kirchheim. Grain refinement in ball-milled nanocrystalline iron in dependence of its oxygen content. DPG-Frühjahrstagung, Dresden (2014).

- M. Trynogga, A. Lehmberg, M. Deutges, C. Borchers, R. Kirchheim. Investigation of crack formation in high-purity iron. 14th International Symposium on MetalHydrogen Systems, Manchester, UK (2014).

- M. Tiegel, A. Lehmberg, M. L. Martin, M. Deutges, C. Borchers, R. Kirchheim. Investigation of crack and blister formation due to hydrogen loading in iron, DPGFrühjahrstagung, Berlin (2015).

- M. C. Tiegel, M. L. Martin, C. Borchers, R. Kirchheim. On Hydrogen-Induced Cracking and Blistering in Iron. International Hydrogen Conference, Wyoming, USA (2016). 


\section{Danksagung}

Ganz herzlich möchte ich mich an dieser Stelle bei allen Personen bedanken, die zum Gelingen meiner Doktorarbeit beigetragen haben.

- Prof. Dr. Reiner Kirchheim danke ich für die Möglichkeit, diese Arbeit zu schreiben. Er stand mir als Doktorvater für Diskussionen und Anregungen stets zur Seite, ermöglichte mir aber auch, eigenständig Ideen zu verwirklichen.

- Für sein Interesse und die Übernahme der Zweitkorrektur meiner Arbeit danke ich Prof. Dr. Michael Seibt.

- Prof. Dr. Astrid Pundt danke ich für Anregungen und Diskussionen. Die Teilnahme an den Meetings ihrer Gruppe halfen bei meiner Arbeit und erweiterten meinen Blick auf Wasserstoff in dünnen Schichten.

- Für ihre langjährige Unterstützung bei meiner Doktorarbeit und die Betreuung meiner Bachelor- und Masterarbeit danke ich Dr. Christine Borchers. Außerdem danke ich ihr für das Korrekturlesen meiner Arbeit und verschiedener Veröffentlichungen.

- Dr. Martin Deutges und May L. Martin, PhD danke ich für die sehr gute Zusammenarbeit während meiner Promotion. Die Diskussionen über meine Arbeit und die experimentelle Zusammenarbeit brachten meine Arbeit voran. Es hat immer sehr viel Spaß gemacht, mit euch zu arbeiten.

- Magnus Hamm danke ich für die Möglichkeit, an seiner Vakuumanlage Messungen durchzuführen. Die Hilfe bei der experimentellen Arbeit und die Diskussionen über diese und weitere Ergebnisse halfen mir sehr.

- Für die Unterstützung bei den FEM-Simulationen danke ich Niklas Teichmann.

- Den weiteren Mitgliedern der Arbeitsgruppen Defactants und Wasserstoff in Metallen verdanke ich viele wissenschaftliche Diskussionen und eine gute Arbeitsatmosphäre.

- Den Laboranten und technischen Mitarbeitern danke ich für eine hervorragende Unterstützung in den Laboren und an verschiedenen Geräten. Besonders möchte ich mich hier bei Annegret Lehmberg, Florian Oelkers-Hilker, Kerstin Born, Karin Ahlborn, Matthias Hahn und Volker Radisch bedanken. 
- Für fleißiges Korrekturlesen möchte ich mich bei Dr. Christine Borchers, Lisa Thom, Juliane Clemens, Dr. Alexander Tiegel, Dr. Martin Deutges und Magnus Hamm bedanken.

- Nicht zuletzt möchte ich mich von Herzen bei meinen Eltern, Geschwistern und besonders bei meinem Ehemann Alexander, für die Unterstützung während meines Studiums und meiner Promotion bedanken.

\section{Vielen Dank!}




\section{Lebenslauf}

\section{Persönliche Daten:}

Name:

Geburtsname:

Geburtstag:

Geburtsort:

\section{Schulausbildung:}

07/1995-07/1999

$07 / 1999-07 / 2001$

$07 / 2001-06 / 2008$

$06 / 2008$

\section{Studium:}

$10 / 2008-09 / 2011$

Bachelorarbeit:

$10 / 2011-10 / 2013$

Masterarbeit:
Marie Christine Tiegel

Trynogga

Oktober 1988

Rotenburg (Wümme)
Nicolaischule Verden

Orientierungsstufe Jahnschule Verden

Domgymnasium Verden

Erwerb der allgemeinen Hochschulreife
Bachelorstudium in Physik an der Universität Göttingen Stabilisierung von nanokristallinem Eisen durch Segregation von Kohlenstoff an den Korngrenzen

Arbeitsgruppe Kirchheim, Institut für Materialphysik Masterstudium in Physik an der Universität Göttingen Untersuchung der Kornverfeinerung von kugelgemahlenem nanokristallinen Eisen in Abhängigkeit der Sauerstoffkonzentration Arbeitsgruppe Kirchheim, Institut für Materialphysik

\section{Promotion:}

$11 / 2013-01 / 2017$

Wissenschaftliche Mitarbeiterin und Promotionsstudentin Arbeitsgruppe Kirchheim, Institut für Materialphysik 


\section{Lehrtätigleiten während der Promotion:}

\begin{tabular}{l|l}
$10 / 2013-03 / 2014$ & $\begin{array}{l}\text { Leitung einer Übungsgruppe zur Vorlesung } \\
\text { Einführung in die Festkörper- und Materialphysik } \\
04 / 2014-08 / 2014\end{array}$ \\
$10 / 2014-03 / 2015$ & $\begin{array}{l}\text { Betreuung des Bachelorstudenten Ferdinand Döring } \\
\text { Leitung einer Übungsgruppe zur Vorlesung } \\
\text { Einführung in die Materialphysik }\end{array}$ \\
$04 / 2015-10 / 2015$ & $\begin{array}{l}\text { Betreuung des Bachelorversuches Transmissionselektronenmikroskopie - } \\
\text { Auf dem Weg zur atomaren Auflösung }\end{array}$ \\
$04 / 2015-09 / 2015$ & $\begin{array}{l}\text { Betreuung des Bachelorstudenten Max Steinbrück } \\
\text { Betreuung des Bachelorversuches } \\
04 / 2015-10 / 2015\end{array}$ \\
$10 / 2015-02 / 2016$ & $\begin{array}{l}\text { Thermodynamik und Phasendiagramm von Legierungen } \\
\text { Betreuung des Bachelorstudenten Nick Šemjatov } \\
04 / 2016-10 / 2016\end{array}$ \\
$10 / 2015-01 / 2017$ & $\begin{array}{l}\text { Betreuung des Bachelorstudenten Jonas Wawra } \\
\text { Betreuung des Materstudenten Yangyi Wang }\end{array}$
\end{tabular}

Göttingen, 25.01.2017 
Literatur

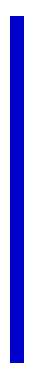





\section{Literatur in alphabetischer Reihenfolge}

[AJ06] P. W. Atkins and L. Jones. Chemie - einfach alles. Wiley-VCH (2006).

$\left[\right.$ AKS $\left.^{+} 14\right]$ E. Ahmad, F. Karim, K. Saeed, T. Manzoor, and G. Zahid. Effect of cold rolling and annealing on the grain refinement of low alloy steel. IOP Conference Series: Materials Science and Engineering 60(1), 012029 (2014).

[BBMN66] W. Beck, J. Bockris, J. McBreen, and L. Nanis. Hydrogen permeation in metals as a function of stress, temperature and dissolved hydrogen concentration. Proceedings of the Royal Society of London A: Mathematical, Physical and Engineering Sciences 290(1421), 220 (1966).

[BDK09] B. Beidokhti, A. Dolati, and A. Koukabi. Effects of alloying elements and microstructure on the susceptibility of the welded HSLA steel to hydrogen-induced cracking and sulfide stress cracking. Materials Science and Engineering: A 507(1-2), 167 (2009).

[Bea72] C. Beachem. A new model for hydrogen-assisted cracking (hydrogen "embrittlement"). Metallurgical Transactions 3(2), 441 (1972).

[Ber70] I. M. Bernstein. Hydrogen-induced cracking in iron: Morphology and crack path dependence. Metallurgical Transactions 1(11), 3143 (1970).

[Ber94] I. Bernstein. The role of hydrogen: Is the story any clearer? Hydrogen effects in materials 5, 3 (1994).

[BHM91] J. Badding, R. Hemley, and H. Mao. High-pressure chemistry of hydrogen in metals- In situ study of iron hydride. Science 253(5018), 421 (1991).

[BS94] H. Birnbaum and P. Sofronis. Hydrogen-enhanced localized plasticity - a mechanism for hydrogen-related fracture. Materials Science and Engineering: A 176(1), 191 (1994).

[BV10] A. Barnoush and H. Vehoff. Recent developments in the study of hydrogen embrittlement: Hydrogen effect on dislocation nucleation. Acta Materialia 58(16), 5274 (2010).

[BW12] T. Bührke and R. Wengenmayr. Erneuerbare Energie: Konzepte für die Energiewende. John Wiley \& Sons (2012).

[CR12] W. D. Callister and D. G. Rethwisch. Materialwissenschaften und Werkstofftechnik: Eine Einführung. John Wiley \& Sons (2012).

[CS93] J. Condon and T. Schober. Hydrogen bubbles in metals. Journal of Nuclear Materials 207, 1 (1993).

[Dö15] F. Döring. Untersuchung der Entstehung von Blistern bei Beladung von reinem Eisen mit Wasserstoff. Bachelorarbeit, Institut für Materialphysik, Georg August Universität Göttingen (2015).

[DAOG01] G. Domizzi, G. Anteri, and J. Ovejero-Garc1a. Influence of sulphur content and inclusion distribution on the hydrogen induced blister cracking in pressure vessel and pipeline steels. Corrosion Science 43(2), 325 (2001). 
$\left[\mathrm{DBC}^{+} 15\right] \quad$ M. Deutges, H. P. Barth, Y. Chen, C. Borchers, and R. Kirchheim. Hydrogen diffusivities as a measure of relative dislocation densities in palladium and increase of the density by plastic deformation in the presence of dissolved hydrogen. Acta Materialia 82, 266 (2015).

$\left[\mathrm{EMD}^{+} 11\right]$ D. P. Escobar, C. Miñambres, L. Duprez, K. Verbeken, and M. Verhaege. Internal and surface damage of multiphase steels and pure iron after electrochemical hydrogen charging. Corrosion Science 53(10), 3166 (2011).

[ER09] M. Elboujdaini and R. W. Revie. Metallurgical factors in stress corrosion cracking (SCC) and hydrogen-induced cracking (HIC). Journal of Solid State Electrochemistry 13(7), 1091 (2009).

[ERWW13] M.-H. Evans, A. Richardson, L. Wang, and R. Wood. Effect of hydrogen on butterfly and white etching crack (WEC) formation under rolling contact fatigue (RCF). Wear 306(1-2), 226 (2013).

[Eur03] European Commission. Hydrogen energy and fuel cells: A vision of our future : [final report of the high level group]. European Commission, Directorate-General for Research (2003).

[Eva12] M.-H. Evans. White structure flaking (WSF) in wind turbine gearbox bearings: effects of 'butterflies' and white etching cracks (WECs). Materials Science and Technology 28(1), 3 (2012).

[FS85] Y. Fukai and H. Sugimoto. Diffusion of hydrogen in metals. Advances in Physics 34(2), 263 (1985).

[Fuk03] Y. Fukai. Formation of superabundant vacancies in $M-H$ alloys and some of its consequences: a review. Journal of Alloys and Compounds 356-357, 263 (2003).

$\left[\mathrm{GDK}^{+} 14\right] \quad$ A. Griesche, E. Dabah, T. Kannengiesser, N. Kardjilov, A. Hilger, and I. Manke. Threedimensional imaging of hydrogen blister in iron with neutron tomography. Acta Materialia 78, 14 (2014).

[Gib28] J. Gibbs. The Collected Works of J. Willard Gibbs. Bd. 2. Yale University Press (1928).

$\left[\mathrm{GKA}^{+} 02\right]$ L. A. Giannuzzi, B. W. Kempshall, S. D. Anderson, B. I. Prenitzer, and T. M. Moore. FIB lift-out for defect analysis. Analysis Techniques of Submicron Defects 2002, 29 (2002).

[Got01] G. Gottstein. Physikalische Grundlagen Der Materialkunde. Springer-Lehrbuch. Springer (2001).

[GR00] H. J. Grabke and E. Riecke. Absorption and diffusion of hydrogen in steels. Materials and Technology 34(6), 331 (2000).

[Haf07] B. Hafner. Scanning electron microscopy primer. Characterization Facility, University of Minnesota-Twin Cities (2007).

[HF13] E. Hayward and C.-C. Fu. Interplay between hydrogen and vacancies in $\alpha-F e$. Physical Review B 87, 174103 (2013).

[Hir80] J. Hirth. Effects of hydrogen on the properties of iron and steel. Metallurgical Transactions A 11(6), 861 (1980).

[HR80] J. P. Hirth and J. R. Rice. On the thermodynamics of adsorption at interfaces as it influences decohesion. Metallurgical Transactions A 11(9), 1501 (1980).

[Irw57] G. Irwin. Analysis of stresses and strains near the end of a crack traversing a plate. Journal of Applied Mechanics 24, 361 (1957). 
[JC04] D. Jiang and E. A. Carter. Diffusion of interstitial hydrogen into and through bcc Fe from first principles. Physical Review B 70(6), 064102 (2004).

[Joh74] W. H. Johnson. On some remarkable changes produced in iron and steel by the action of hydrogen and acids. Proceedings of the Royal Society of London 23(156-163), 168 (1874).

[Kir07a] R. Kirchheim. Reducing grain boundary, dislocation line and vacancy formation energies by solute segregation. I. Theoretical background. Acta Materialia 55(15), 5129 (2007).

[Kir07b] R. Kirchheim. Reducing grain boundary, dislocation line and vacancy formation energies by solute segregation: II. Experimental evidence and consequences. Acta Materialia 55(15), 5139 (2007).

[Kir11] R. Kirchheim. Hydrogen trapping and its effect on permeation and thermal desorption. Steely Hydrogen Conference Proceedings pages 286-294 (2011).

[KJ80] A. Kumnick and H. Johnson. Deep trapping states for hydrogen in deformed iron. Acta Metallurgica 28(1), 33 (1980).

[KSS15] R. Kirchheim, B. Somerday, and P. Sofronis. Chemomechanical effects on the separation of interfaces occurring during fracture with emphasis on the hydrogen-iron and hydrogen-nickel system. Acta Materialia 99, 87 (2015).

$\left[\mathrm{KTA}^{+}{ }^{14}\right] \quad$ M. Koyama, C. C. Tasan, E. Akiyama, K. Tsuzaki, and D. Raabe. Hydrogen-assisted decohesion and localized plasticity in dual-phase steel. Acta Materialia 70, 174 (2014).

[Lan01] G. Lange. Systematische Beurteilung technischer Schadensfälle. John Wiley \& Sons (2001).

[Leh14] A. Lehmberg. Untersuchung der Volumenänderung von Eisen nach Wasserstoffbeladung. Bachelorarbeit, Hochschule für angewandte Wissenschaft und Kunst, Göttingen (2014).

[Len09] Y. Leng. Materials characterization: introduction to microscopic and spectroscopic methods. John Wiley \& Sons (2009).

[LL86] J.-Y. Lee and S. Lee. Hydrogen trapping phenomena in metals with B.C.C. and F.C.C. crystals structures by the desorption thermal analysis technique. Surface and Coatings Technology 28(3), 301 (1986).

[LP16] U. Lenk and I. Pyc. Flexibilisierung durch Hybridisierung der Energieversorgung. e \& i Elektrotechnik und Informationstechnik 133(4), 216 (2016).

[LPV16] A. Laureys, R. Petrov, and K. Verbeken. Internal and surface damage after electrochemical hydrogen charging for ultra low carbon steel with various degrees of recrystallization. Procedia Structural Integrity, 21st European Conference on Fracture 2, 541 (2016).

[Lyn12] S. Lynch. Hydrogen embrittlement phenomena and mechanisms. Corrosion Reviews 30(3-4), 105 (2012).

[MF63] A. McNabb and P. Foster. A new analysis of the diffusion of hydrogen in iron and ferritic steels. Transactions of the Metallurgical Society of AIME 227(3), 618 (1963).

$\left[\mathrm{MFL}^{+} 11\right] \quad$ M. L. Martin, J. A. Fenske, G. S. Liu, P. Sofronis, and I. M. Robertson. On the formation and nature of quasi-cleavage fracture surfaces in hydrogen embrittled steels. Acta Materialia $\mathbf{5 9}(4), 1601$ (2011).

[MG12] K. Mazloomi and C. Gomes. Hydrogen as an energy carrier: Prospects and challenges. Renewable and Sustainable Energy Reviews 16(5), 3024 (2012). 
$\left[\mathrm{MKT}^{+} 16\right] \quad$ E. Merson, A. Kudrya, V. Trachenko, D. Merson, V. Danilov, and A. Vinogradov. Quantitative characterization of cleavage and hydrogen-assisted quasi-cleavage fracture surfaces with the use of confocal laser scanning microscopy. Materials Science and Engineering: A 665, 35 (2016).

[MRS11] M. Martin, I. Robertson, and P. Sofronis. Interpreting hydrogen-induced fracture surfaces in terms of deformation processes: A new approach. Acta Materialia 59(9), 3680 (2011).

[MS84] M. Moser and V. Schmidt. Fractography and mechanism of hydrogen cracking-the fisheye concept. Advances in Fracture Researchb (Fracture 84). 4, 2459 (1984).

[MS07] T. Maitland and S. Sitzman. Electron backscatter diffraction (EBSD) technique and materials characterization examples. Springer Berlin (2007).

[Nag16] M. Nagumo. Fundamentals of hydrogen embrittlement. Springer (2016).

[NB78] F. Nakasato and I. M. Bernstein. Crystallographic and fractographic studies of hydrogeninduced cracking in purified iron and iron- silicon alloys. Metallurgical Transactions A 9(9), 1317 (1978).

[NC92] S. Nakahara and A. Cullis. Simple method for determining the absolute sense of image rotation in a transmission electron microscope. Ultramicroscopy 45(3), 365 (1992).

[Ori72] R. A. Oriani. A mechanistic theory of hydrogen embrittlement of steels. Berichte der Bunsengesellschaft für physikalische Chemie 76, 848 (1972).

[OVB93] M. Overwijk, F. Van den Heuvel, and C. Bulle-Lieuwma. Novel scheme for the preparation of transmission electron microscopy specimens with a focused ion beam. Journal of Vacuum Science \& Technology B 11(6), 2021 (1993).

[PBP16] A. Pineau, A. A. Benzerga, and T. Pardoen. Failure of metals I: Brittle and ductile fracture. Acta Materialia 107, 424 (2016).

[Pet58] N. Petch. The ductile-brittle transition in the fracture of $\alpha$-iron: I. Philosophical Magazine 3(34), 1089 (1958).

[Pfe26] L. B. Pfeil. The effect of occluded hydrogen on the tensile strength of iron. Proceedings of the Royal Society of London A: Mathematical, Physical and Engineering Sciences 112(760), $182(1926)$.

$\left[\mathrm{PGL}^{+} 13\right]$ L. T. Popoola, A. S. Grema, G. K. Latinwo, B. Gutti, and A. S. Balogun. Corrosion problems during oil and gas production and its mitigation. International Journal of Industrial Chemistry 4(1), 1 (2013).

[Pun04] A. Pundt. Hydrogen in nano-sized metals. Advanced Engineering Materials 6(1-2), 11 (2004).

[QJ78] N. Quick and H. Johnson. Hydrogen and deuterium in iron, 49-506 ${ }^{\circ}$. Acta Metallurgica 26(6), 903 (1978).

$\left[\mathrm{RCL}^{+} 08\right]$ X. Ren, W. Chu, J. Li, Y. Su, and L. Qiao. The effects of inclusions and second phase particles on hydrogen-induced blistering in iron. Materials Chemistry and Physics 107(2-3), 231 (2008).

$\left[\right.$ RFM $\left.^{+} 09\right]$ I. M. Robertson, J. Fenske, M. Martin, M. Briceno, M. Dadfarnia, P. Novak, D. C. Ahn, P. Sofronis, J. B. Liu, and D. D. Johnson. Understanding how hydrogen influences the mechanical properties of iron and steel. Proceedings of the 2nd International Symposium on Steel Science (2009). 
[RHB06] J. Rösler, H. Harders, and M. Bäker. Mechanisches Verhalten der Werkstoffe. Springer (2006).

[Rob01] I. Robertson. The effect of hydrogen on dislocation dynamics. Engineering Fracture Mechanics 68(6), 671 (2001).

$\left[\mathrm{RSN}^{+} 15\right]$ I. M. Robertson, P. Sofronis, A. Nagao, M. Martin, S. Wang, D. Gross, and K. Nygren. Hydrogen embrittlement understood. Metallurgical and Materials Transactions B 46(3), 1085 (2015).

$\left[\mathrm{RZC}^{+} 07\right]$ X. Ren, Q. Zhou, W. Chu, J. Li, Y. Su, and L. Qiao. The mechanism of nucleation of hydrogen blister in metals. Chinese Science Bulletin 52(14), 2000 (2007).

$\left[\mathrm{RZS}^{+} 08\right]$ X. Ren, Q. Zhou, G. Shan, W. Chu, J. Li, Y. Su, and L. Qiao. A nucleation mechanism of hydrogen blister in metals and alloys. Metallurgical and Materials Transactions A 39(1), 87 (2008).

[SF14] H. Sugimoto and Y. Fukai. Hydrogen-induced superabundant vacancy formation in bcc Fe: Monte Carlo simulation. Acta Materialia 67, 418 (2014).

[SFAdA08] J. Sanchez, J. Fullea, C. Andrade, and P. L. de Andres. Hydrogen in $\alpha$-iron: Stress and diffusion. Physical Review B 78, 014113 (2008).

[SI71] G. Spink and M. Ives. Morphology of crystallographic etch pits in iron. Journal of Applied Physics 42(2), 511 (1971).

[Sie29] A. Sieverts. Die Aufnahme von Gasen durch Metalle. Zeitschrift für Metallkunde 21(2), 37 (1929).

$\left[\mathrm{SKH}^{+} 11\right] \quad$ H. Suzuki, D. Kobayashi, N. Hanada, K. Takai, and Y. Hagihara. Existing state of hydrogen in electrochemically charged commercial-purity aluminum and its effects on tensile properties. Materials Transactions 52(9), 1741 (2011).

[SMM90] A. San-Martin and F. D. Manchester. The Fe-H (Iron-Hydrogen) system. Bulletin of Alloy Phase Diagrams 11(2), 173 (1990).

[SRB88] D. Shih, I. Robertson, and H. Birnbaum. Hydrogen embrittlement of $\alpha$ titanium: In situ tem studies. Acta Metallurgica 36(1), 111 (1988).

$\left[\mathrm{SSR}^{+} 13\right] \quad$ E. Shekari, M. R. Shishesaz, G. Rashed, M. Farzam, and E. Khayer. Failure investigation of hydrogen blistering on low-strength carbon steel. Iranian Journal of Oil \& Gas Science and Technology 2(2), 65 (2013).

[Sta97] Stahlinstitut VDEh, editor. Erscheinungsformen von Rissen und Brüchen metallischer Werkstoffe-The appearance of cracks and fractures in metallic materials. Stahleisen Ver$\operatorname{lag}(1997)$.

[Sto16] D. Stolten. Hydrogen science and engineering: Materials, processes, systems and technology. John Wiley \& Sons (2016).

[STTY11] V. M. Schastlivtsev, T. I. Tabatchnikova, N. A. Tereshchenko, and I. L. Yakovleva. Degradation of the pipe-steel structure upon long-term operation in contact with a hydrogen sulfide-containing medium. The Physics of Metals and Metallography 111(3), 281 (2011).

[Tap13] D. Taplin. The physical metallurgy of fracture: Fourth International conference on fracture, June 1977, University of Waterloo, Canada. Elsevier Science (2013). 
[TML $\left.{ }^{+} 16\right]$ M. C. Tiegel, M. L. Martin, A. K. Lehmberg, M. Deutges, C. Borchers, and R. Kirchheim. Crack and blister initiation and growth in purified iron due to hydrogen loading. Acta Materialia 115, 24 (2016).

[TR61] A. Tetelman and W. Robertson. The mechanism of hydrogen embrittlement observed in iron-silicon single crystals. Technical report, DTIC Document (1961).

[WAM87] M. Wada, N. Akaiwa, and T. Mori. Field evaporation of iron in neon and in hydrogen and its rate-controlling processes. Philosophical Magazine A 55(3), 389 (1987).

[Waw16] J. Wawra. Veränderungen der mechanischen Eigenschaften unter Wasserstoffbeladung am Modellsystem Ta und Fe. Bachelorarbeit, Institut für Materialphysik, Georg August Universität Göttingen (2016).

[Wes39] H. Westergaard. Bearing pressures and cracks. Journal of applied mechanics 18 (1939).

[Wes69] D. G. Westlake. Generalized model for hydrogen embrittlement. Technical report, Argonne National Laboratory, Illinois (1969).

[Wip97] H. Wipf. Diffusion of hydrogen in metals. Hydrogen in metals III pages 51-91 (1997).

[Wip01] H. Wipf. Solubility and diffusion of hydrogen in pure metals and alloys. Physica Scripta 2001(T94), 43 (2001).

[Züt04] A. Züttel. Hydrogen storage methods. Naturwissenschaften 91(4), 157 (2004). 